\title{
Labor Mobility in a Monetary Union
}

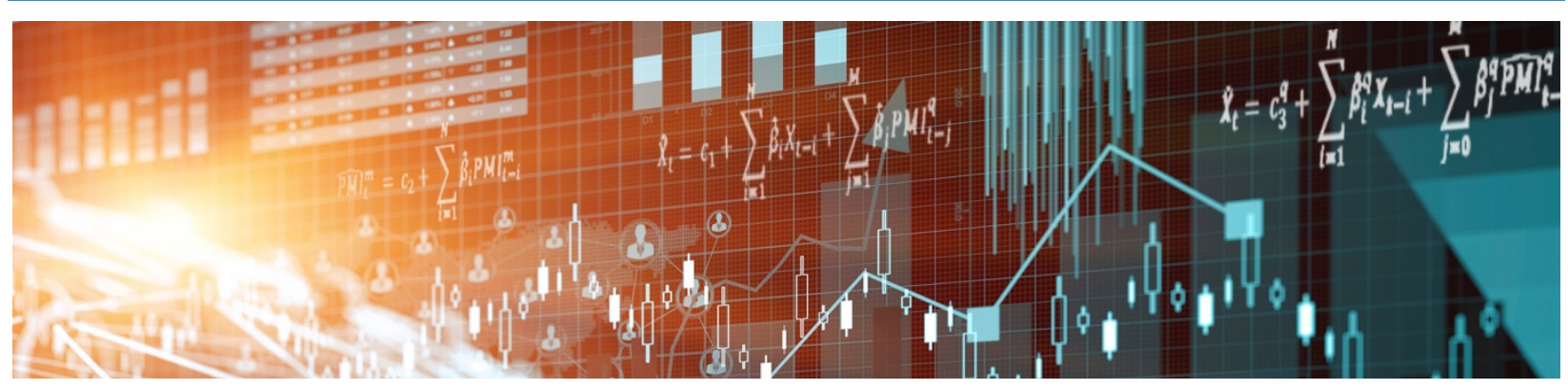

by Daniela Hauser and Martin Seneca 
Bank of Canada Staff Working Paper 2019-15

April 2019

\title{
Labor Mobility in a Monetary Union
}

\author{
by
}

\author{
Daniela Hauser ${ }^{1}$ and Martin Seneca ${ }^{2}$ \\ ${ }^{1}$ Canadian Economic Analysis Department \\ Bank of Canada \\ Ottawa, Ontario, Canada K1A 0G9 \\ dhauser@bankofcanada.ca \\ 2 Monetary Assessment and Strategy Division \\ Bank of England \\ London, United Kingdom EC2R 8AH \\ martin.seneca@bankofengland.co.uk
}




\section{Acknowledgements}

We thank seminar participants at the Bank of Canada, the Bank of England, the 2018 ASSA Annual Meeting in Philadelphia, the 2018 CEF conference in Milan, the 2018 EEA Annual Congress in Cologne, the 2018 Workshop on the Macroeconomics of Migration in Sheffield, the University of Florida, and the 2019 SNDE Conference in Dallas. The views expressed in this paper are solely those of the authors and do not necessarily reflect those of the Bank of Canada and the Bank of England or any of its policy committees. 


\begin{abstract}
The optimal currency literature has stressed the importance of labor mobility as a precondition for the success of monetary unions. But only a few studies formally link labor mobility to macroeconomic adjustment and policy. In this paper, we study macroeconomic dynamics and optimal monetary policy in an economy with cyclical labor flows across two distinct regions that share trade links and a common monetary framework. In our New Keynesian dynamic, stochastic, general-equilibrium model calibrated to the United States, migration flows are driven by fluctuations in the relative labor market performance across the monetary union. While labor mobility can be an additional channel for cross-regional spillovers as well as a regional shock absorber, we find that a mobile labor force closes the efficiency gaps in the labor market and thus lessens the trade-off between inflation and labor market stabilization. As migration flows are generally inefficient, however, regionspecific disturbances introduce additional trade-offs with regional labor market conditions. Putting some weight on stabilizing fluctuations in the labor market enhances welfare when monetary policy follows a simple rule.
\end{abstract}

Bank topics: Business fluctuations and cycles; Labour markets; Economic models;

Monetary policy framework; Regional economic developments

JEL codes:E32, E52, F45

\title{
Résumé
}

La littérature consacrée aux zones monétaires optimales met en évidence l'importance de la mobilité de la main-d'œuvre comme condition préalable au succès d'une union monétaire. Toutefois, seulement quelques études établissent un lien formel entre la mobilité de la main-d'œuvre et les ajustements et politiques macroéconomiques. Dans cette étude, nous nous penchons sur les dynamiques macroéconomiques et la politique monétaire optimale dans une économie où des flux cycliques de main d'œuvre s'opèrent entre deux régions distinctes ayant des liens commerciaux ainsi qu'un cadre monétaire commun. Dans notre modèle d'équilibre général dynamique et stochastique (EGDS) néokeynésien calibré sur des données des États-Unis, les flux migratoires sont fonction de la tenue relative du marché du travail dans l'ensemble de l'union monétaire. Bien que la mobilité de la maind'œuvre puisse amplifier les inefficiences transrégionales, elle permet aussi d'amortir les chocs régionaux. Nous constatons également que la mobilité comble les écarts d'efficience sur le marché du travail ce qui réduit, en conséquence, l'arbitrage entre l'inflation et la stabilisation de ce marché. Cependant, les flux migratoires étant généralement inefficaces, 
les chocs régionaux entraînent des compromis supplémentaires attribuables aux conditions du marché du travail régional. Le fait d'accorder une certaine importance à la stabilisation des fluctuations du marché du travail améliore le bien-être lorsque la politique monétaire suit une règle simple.

Sujets : Cycles et fluctuations économiques; Marchés du travail; Modèles économiques; Cadres de la politique monétaire; Évolution économique régionale

Codes JEL : E32, E52, F45 


\section{Non-technical summary}

Geographical mobility of workers has long been considered an important adjustment to economic disturbances. The intuition is that factor mobility may cushion the effects of region-specific shocks when conventional stabilization mechanisms, such as monetary policy, are unavailable. Surprisingly, theories of macroeconomic fluctuations often overlook the role of labor mobility across different regions of a monetary union. This paper studies macroeconomic dynamics and optimal monetary policy in an economy with cyclical labor flows across two distinct regions sharing trade links and a common monetary framework.

To this end, we develop a two-region dynamic stochastic general-equilibrium model with endogenous labor mobility and standard frictions in both regional labor markets as well as in firms' pricing decisions. Migration flows are driven by fluctuations in the relative labor market performance across the monetary union. We show that households do not fully internalize the effects of their migration decisions on aggregate labor market outcomes, such as wages or unemployment. This new distortion stemming from inefficient migration decisions gives rise to an additional trade-off between differences in labor market conditions across the union. Thus, a mobile labor force requires the policymaker who is optimizing for social welfare to show greater flexibility in inflation targeting by also leaning against inefficient migration flows.

Turning to macroeconomic dynamics, we use our model to show how labor mobility nevertheless facilitates macroeconomic adjustments, improves welfare and eases the burden on monetary policy. Hence, the social benefits stemming from a mobile labor force turn out to be significant, even when households do not take full account of them when making individual migration decisions. However, ignoring labor mobility - and specifically the additional trade-offs related to households' migration decisionspotentially comes at an extra welfare cost. For example, welfare losses associated to strict inflation targeting are bigger in our model with labor mobility compared with the same model with an immobile labor force. In general, a mobile labor force alleviates some of the side effects of suboptimal monetary policy by closing inefficient gaps in regional labor markets through migration. Migration flows thus lessen the trade-off between inflation and labor-market stabilization and strengthen the case for leaning against labor market imbalances in the monetary union. A simple inflation-targeting rule with a moderate weight on unemployment comes close to minimizing welfare losses in the monetary union. 


\section{Introduction}

The optimal currency area literature in the tradition of Mundell (1961) has stressed the importance of labor mobility as a pre-condition for monetary unions (see e.g. Dellas and Tavlas, 2009, for a survey). The intuition is that factor mobility may cushion the effects of region-specific shocks when conventional stabilization mechanisms are unavailable. But only a few studies formally link labor mobility to macroeconomic adjustment and policy. In this paper, we seek to fill this gap in the literature by studying macroeconomic dynamics and (optimal) monetary policy in a model economy with cyclical labor flows across distinct regions that share trade links and a common monetary policy.

The paper makes three key contributions. First, we establish a benchmark for our analysis by deriving optimal monetary policy in a New Keynesian dynamic, stochastic, general-equilibrium (DSGE) model of a monetary union in which employment is determined through a search and matching process in regional labor markets. In doing so, we combine optimal monetary policy in a monetary union (as e.g. in Benigno, 2004) with optimal monetary policy in economies with labor market frictions (as e.g. in Thomas, 2008; Blanchard and Galí, 2010; Ravenna and Walsh, 2011). Second, we derive optimal migration flows within the monetary union. In our full model with free labor movements, households allocate workers across the union to equalize expected net gains from participation in each regional labor market. Hence, migration flows are driven by fluctuations in the relative labor market performance consistent with the empirical evidence (e.g. Saks and Wozniak, 2011; Lkhagvasuren, 2012; Lewis and Swannel, 2018-729). Third, we derive optimal monetary policy responses to these migration flows within the union. As for our benchmark economy, we obtain a loss function for the common monetary authority from a second-order approximation of the welfare of households across regions, expressed in terms of efficiency gaps.

Like Benigno (2004), we find that the optimizing policymaker faces a trade-off between producer price stability in individual regions and the appropriate allocation of demand across the monetary union whenever region-specific shocks call for adjustments in relative prices. In line with results in, for example, Ravenna and Walsh (2011) for the closed economy, labor market frictions give rise to additional trade-offs with labor market conditions in regional labor markets. Moreover, as households do not fully internalize the effects of their migration decisions on aggregate labor market outcomes, labor mobility implies that region-specific disturbances induce further trade-offs between differences in labor market conditions across the union. Thus, free movement of labor complicates the setting of monetary policy and requires the optimizing policymaker to show greater flexibility in inflation targeting by also leaning against small deviations of migration flows from their efficient benchmark. ${ }^{1}$

\footnotetext{
${ }^{1}$ See e.g. Walsh (2014) for a discussion of the appropriate degree of flexibility in inflation targeting based on the existing literature on economies with openness or labor market frictions.
} 
Turning to macroeconomic dynamics, we use our model to show how labor mobility nevertheless facilitates macroeconomic adjustments in a monetary union. We illustrate how the role played by labor mobility may depend on the underlying macroeconomic disturbances, the structure of regional economies, and the monetary policy regime. For example, labor mobility allows a region to effectively export some of the unemployment that would otherwise be associated with region-specific technological advances when prices are sticky and monetary policy accommodation is insufficient. At the same time, the rest of the union may import workers to avoid overheating in regional labor markets caused by a looser common monetary policy stance. Labor mobility makes up for some of the shortcomings of the common monetary policy in this case. By contrast, optimal monetary policy supports aggregate demand enough to avoid a deterioration in labor market conditions in the region exposed to the shock, inducing an appropriate flow of inward migration to employ more workers where they are most productive. Monetary policy and labor mobility now pull together to improve macroeconomic outcomes across the union.

Finally, we consider the welfare consequences of following a set of suboptimal simple monetary policy rules instead of the optimal policy prescriptions. ${ }^{2}$ A strict inflationtargeting regime, for example, leads to higher welfare costs relative to optimal policy when labor is free to cross regional borders. In this case, a simple rule leaning against both inflation and unemployment can deliver smaller welfare losses. But strict inflationtargeting remains close to optimal; the extra degree of flexibility in inflation targeting called for by inefficiencies in households' migration decisions turns out to be quantitatively small. More broadly, labor mobility reduces welfare costs of following simple instrument rules as migration flows help to close inefficiency gaps in regional labor markets when monetary policy is suboptimal. The social benefits to free movement turn out to be significant, even when households do not take full account of them when making individual migration decisions.

Our paper follows the previous study of labor mobility in a monetary union by Farhi and Werning (2014-20105). Compared to their simple static framework, however, we place our analysis within a fully articulated DSGE model with search and matching frictions in labor markets and a range of macroeconomic disturbances. Moreover, we fully characterize and solve for optimal monetary policy from the perspective of social welfare. The contemporaneous work by House et al. (2018) similarly builds a search model with migration. These authors formally evaluate Mundell (1961)'s conjecture that factor mobility serves as a substitute for independent monetary policy, showing that greater labor migration and exchange rate adjustments reduce crosssectional dispersion in unemployment and GDP, but differ along other dimensions, such as differentials in output and inflation. More broadly, the paper relates to a

\footnotetext{
${ }^{2}$ We consider four different rules: a regime of strict inflation-targeting, a simple instrument rule leaning against inflation, a simple rule leaning against both inflation and unemployment, and a rule with an interest-rate smoothing and weights on inflation and output.
} 
growing literature on the role of migration in macroeconomic adjustments. Bandeira et al. (2018), for example, investigate the interplay between fiscal consolidations, and migration, and Smith and Thoenissen (2018) analyze how relative human capital levels shape the business-cycle effects of migration flows on the economy.

The paper is organized as follows. Section 2 lays out the model. The efficient allocation is characterized and compared to the market equilibrium in Section 3, while the optimal policy is derived in Section 4. Section 5 presents the calibration to U.S. data. Section 6 contains a description of macroeconomic dynamics following disturbances to supply, demand, and labor market efficiency. Welfare results are reported in Section 7, and concluding remarks in Section 8.

\section{The model economy}

The model economy is composed of two regions $i$, and $j$, sharing a common monetary authority. In each region, households consume and enjoy leisure. A share of workers are employed either at home as native workers or across the regional border as migrant workers. At the beginning of each period, households allocate those workers that are not currently in employment to the two labor markets. Here, wages are settled with firms through a wage bargaining process. Regional intermediate firms hire new workers from the pool of native and migrant job searchers before selling their products to retailers in a competitive wholesale market. Retailers turn intermediate products into marketable goods for final use in the economy. They operate under monopolistic competition and set prices subject to a nominal rigidity. The common monetary authority set the nominal short-term interest rate for the whole union.

\subsection{Households}

The representative household in region $i$ consists of a continuum of family members of mass 1 . During any given period $t$, individual members are employed or unemployed either in labor market $i\left(E_{t}^{i i}\right.$ and $\left.U_{t}^{i i}\right)$ or in labor market $j\left(E_{t}^{i j}\right.$ and $\left.U_{t}^{i j}\right)$ so that $E_{t}^{i i}+U_{t}^{i i}+E_{t}^{i j}+U_{t}^{i j}=1$. When a period ends, a fraction $\rho$ of the employed in both regions are separated from their jobs. At the beginning of each period, the total mass of household $i$ members searching for a job is given by those members who spent the previous period as unemployed as well as those who have just lost their jobs, in either of the two labor markets:

$$
\begin{aligned}
S_{H, t}^{i} & =U_{t-1}^{i i}+\rho E_{t-1}^{i i}+U_{t-1}^{i j}+\rho E_{t-1}^{i j} \\
& =1-(1-\rho)\left[E_{t-1}^{i i}+E_{t-1}^{i j}\right] .
\end{aligned}
$$

We assume that the household allocates its pool of searchers to each of the two labor markets, regardless of their previous migration status. Specifically, taken labor market conditions as given, the household keeps a fraction $\Omega_{t}^{i}$ of its searchers at home to look for jobs in region $i, S_{t}^{i i}=\Omega_{t}^{i} S_{H, t}^{i}$, while the remaining searchers are sent as migrants to seek employment in region $j, S_{t}^{i j}=\left(1-\Omega_{t}^{i}\right) S_{H, t}^{i}$. Once the migration decision has been 
made, members can search in their designated regional labor market only. If they fail to find a job in that particular market, they spend the period there in unemployment. Assuming instantaneous hiring, the evolution of employment of household $i$ members as native workers in the home labor market becomes

$$
E_{t}^{i i}=(1-\rho) E_{t-1}^{i i}+M_{t}^{i i}=(1-\rho) E_{t-1}^{i i}+f_{t}^{i i} S_{t}^{i i}
$$

where $M_{t}^{i i}$ denotes the number of successful matches of job searchers, $S_{t}^{i i}$, to vacancies in region $i$, and $f_{t}^{i i} \equiv M_{t}^{i i} / S_{t}^{i i}$ is the job-finding rate. Similarly, the evolution of employment of household $i$ migrants in region $j$ becomes

$$
E_{t}^{i j}=(1-\rho) E_{t-1}^{i j}+f_{t}^{i j} S_{t}^{i j}
$$

with $f_{t}^{i j} \equiv M_{t}^{i j} / S_{t}^{i j}$. Corresponding unemployment rates are

$$
U_{t}^{i i} \equiv S_{t}^{i i}-M_{t}^{i i}=\left(1-f_{t}^{i i}\right) S_{t}^{i i} ; \quad U_{t}^{i j} \equiv\left(1-f_{t}^{i j}\right) S_{t}^{i j} .
$$

For convenience, we assume that family members consume and enjoy leisure in the region of their origin and that idiosyncratic income risk is perfectly insured away within the family. ${ }^{3}$ In period $t$ household $i$ chooses consumption, $C_{t}^{i}$, savings in the form of a portfolio of state-contingent zero-coupon nominal bonds, $D_{t+1}^{i}$, as well as the share of searchers remaining in the home labor market, $\Omega_{t}^{i}$, to maximize expected lifetime utility, which we specify in the following form:

$$
\mathbf{E}_{\mathbf{t}} \sum_{t=0}^{\infty} \beta^{t} \mathcal{U}_{t}^{i}=\mathbf{E}_{\mathbf{t}} \sum_{t=0}^{\infty} \beta^{t} \frac{\left[\left(C_{t}^{i}\right)^{b}\left(l_{t}^{i}\right)^{1-b}\right]^{1-\sigma}-1}{1-\sigma}
$$

with

$$
\begin{aligned}
1-l_{t}^{i} & =\left[\left(1-\alpha_{1}\right)^{1 / \nu_{1}}\left(E_{t}^{i i}+\zeta U_{t}^{i i}\right)^{\frac{\nu_{1}-1}{\nu_{1}}}+\left(\alpha_{1}\right)^{1 / \nu_{1}}\left(E_{t}^{i j}+\zeta U_{t}^{i j}\right)^{\frac{\nu_{1}-1}{\nu_{1}}}\right]^{\frac{\nu_{1}}{\nu_{1}-1}} \\
C_{t}^{i} & =\left[\left(1-\alpha_{2}\right)^{1 / \nu_{2}}\left(C_{H, t}^{i}\right)^{\frac{\nu_{2}-1}{\nu_{2}}}+\left(\alpha_{2}\right)^{1 / \nu_{2}}\left(C_{F, t}^{i}\right)^{\frac{\nu_{2}-1}{\nu_{2}}}\right]^{\frac{\nu_{2}}{\nu_{2}-1}}
\end{aligned}
$$

where $1-l_{t}^{i}$ captures the utility cost in terms of the time spent on labor market activities in either of the two labor markets. This disutility is assumed to be a CES aggregator of family members' labor market activities in regions $i$ and $j$, where $\nu_{1}<0$ measures the elasticity of substitution between being a native member of the labor force in region $i$ and being a migrant member of the labor force in labor market $j$, and $\alpha_{1}$ captures migration costs in the form of differences in the disutility attached to working or searching in either of the two regions. As discussed in further detail

\footnotetext{
${ }^{3}$ Considerations relative to the location and type of consumption of different workers, as discussed in Farhi and Werning (2014-20105), are thus abstracted from.
} 
in Section 5, $\alpha_{1}$ pins down the steady-state share of migrant (un)employment to total (un)employment as well as the share of migrant searchers in the entire pool of searchers. $\zeta$ captures time spent on labor market activities by the unemployed, such as searching for jobs, developing skills or collecting unemployment benefits. Similarly, aggregate consumption is measured by a CES aggregator of domestic and imported consumption goods, each in turn given by a CES function,

$$
C_{H, t}^{i} \equiv\left[\int_{0}^{1} C_{H, t}^{i}(l)^{\frac{\varepsilon-1}{\varepsilon}} d l\right]^{\frac{\varepsilon}{\varepsilon-1}} ; \quad C_{F, t}^{i} \equiv\left[\int_{0}^{1} C_{F, t}^{i}(l)^{\frac{\varepsilon-1}{\varepsilon}} d l\right]^{\frac{\varepsilon}{\varepsilon-1}}
$$

where $l \in[0,1]$ denotes the consumption good variety, assuming that each country produces a continuum of differentiated final goods. The parameter $\alpha_{2}$ reflects the openness of the regional economy and is inversely related to the degree of home bias in consumption, while $\nu_{2}$ determines the elasticity of substitution between domestic and foreign goods. Total consumption expenditures of the representative household in $i$ is given by $P_{H, t}^{i} C_{H, t}^{i}+P_{F, t}^{i} C_{F, t}^{i}=P_{t}^{i} C_{t}^{i}$, where $P_{H, t}^{i}$ and $P_{F, t}^{i}$ are indices of domestically produced and imported goods, respectively, and $P_{t}^{i}$ is the consumer price index (CPI). Hence, households maximize utility subject to the following budget constraint:

$$
P_{t}^{i} C_{t}^{i}+\mathbf{E}_{\mathbf{t}}\left(\mathcal{Q}_{t, t+1} D_{t+1}^{i}\right) \leq D_{t}^{i}+W_{t}^{i i} E_{t}^{i i}+P_{t}^{i} b^{U} U_{t}^{i i}+W_{t}^{i j} E_{t}^{i j}+P_{t}^{j} b^{U} U_{t}^{i j}+T_{t}^{i} .
$$

The employed earn a wage $W_{t}^{i i}$ as natives and $W_{t}^{i j}$ as migrants, and the unemployed are entitled to unemployment benefits $\left(b^{U}\right) \cdot \mathcal{Q}_{t, t+1}$ is the stochastic discount factor, and $T_{t}^{i}$ are lump-sum taxes and transfers in region $i$. The first-order conditions with respect to consumption, $C_{t}^{i}$, and the zero-coupon bond, $D_{t+1}^{i}$, yields a standard Euler equation,

$$
\beta R_{t} \mathbf{E}_{\mathbf{t}}\left\{\left(\frac{U_{c, t+1}^{i}}{U_{c, t}^{i}}\right)\left(\frac{1}{\Pi_{t+1}^{i}}\right)\right\}=1
$$

where $\Pi_{t+1}^{i} \equiv P_{t+1}^{i} / P_{t}^{i}$ captures CPI inflation in region $i$ and where $U_{c, t}^{i}$ denotes the marginal utility of consumption of household $i$ in period $t{ }^{4}$ For the given utility specification (5), the marginal utility of consumption states as

$$
U_{c, t}^{i}=\left[\left(C_{t}^{i}\right)^{b}\left(l_{t}^{i}\right)^{1-b}\right]^{-\sigma} b\left(\frac{C_{t}^{i}}{l_{t}^{i}}\right)^{b-1} Z_{t}^{i}
$$

Here, $Z_{t}^{i}$ is a preference shock, where

$$
\log Z_{t}^{i}=\rho_{z} \log Z_{t-1}^{i}+\nu_{z, t}^{i}
$$

\footnotetext{
${ }^{4}$ No arbitrage implies the risk-free, union-wide interest rate to be defined by $R_{t}^{-1}=\mathbf{E}_{\mathbf{t}}\left\{\mathcal{Q}_{t, t+1}\right\}$.
} 
and $\nu_{z, t}^{i}$ is i.i.d. with zero mean and variance $\sigma_{z}^{2}$. Under the assumption of complete markets for securities traded across the two geographical regions, a standard risksharing condition applies,

$$
U_{c, t}^{i} Q_{t}^{i}=U_{c, t}^{j} \vartheta
$$

where $Q_{t}^{i} \equiv P_{t}^{j} / P_{t}^{i}$ is the real exchange rate (from the point of view of region $i$ ) and $\vartheta$ is a constant that depends on initial conditions. The first-order condition with respect to $\Omega_{t}^{i}$, derived under the assumption that households take both job-finding rates and real wages as given, provides a condition determining the optimal migration flows: ${ }^{5}$

$$
\begin{aligned}
& f_{t}^{i i} \frac{W_{t}^{i i}}{P_{t}^{i}}+\left(1-f_{t}^{i i}\right) b^{U}-\frac{U_{l, t}^{i}}{U_{c, t}^{i}}\left[\frac{\left(1-\alpha_{1}\right)\left(1-l_{t}^{i}\right)}{E_{t}^{i i}+\zeta U_{t}^{i i}}\right]^{1 / \nu_{1}}\left[f_{t}^{i i}+\left(1-f_{t}^{i i}\right) \zeta\right]= \\
& f_{t}^{i j} \frac{W_{t}^{i j}}{P_{t}^{j}} Q_{t}^{i}+\left(1-f_{t}^{i j}\right) b^{U}-\frac{U_{l, t}^{i}}{U_{c, t}^{i}}\left[\frac{\alpha_{1}\left(1-l_{t}^{i}\right)}{E_{t}^{i j}+\zeta U_{t}^{i j}}\right]^{1 / \nu_{1}}\left[f_{t}^{i j}+\left(1-f_{t}^{i j}\right) \zeta\right],
\end{aligned}
$$

where $U_{l, t}^{i}$ is the marginal utility of leisure. This condition states that the household allocates searchers to the two labor markets so that the respective expected net benefits from participating in them are equalized. With probability $f_{t}^{i i}$, a searcher in region $i$ is matched to a vacancy and earns a real wage, $W_{t}^{i i} / P_{t}^{i}$. With the complement probability, the searcher does not find a job and is left with the unemployment benefit $b^{U}$. The expected net benefit from sending an extra searcher to region $i$ is therefore the probability weighted sum of the real wage and the unemployment benefit in excess of the expected utility cost of labor market activities in region $i$. These activities require one unit of time if employed (with probability $f_{t}^{i i}$ ) and $\zeta$ units if unemployed (with probability $1-f_{t}^{i i}$ ), and are evaluated at the household's marginal rate of substitution between consumption and leisure when participating in region $i$ 's labor market. ${ }^{6}$ If this expected benefit on the left-hand side of (14) were not equal to the expected benefit from participation in labor market $j$ on the right-hand side, the household could increase its expected utility by sending more searchers to the labor market with the highest expected net benefit.

\subsection{Intermediate production}

Intermediate firms in region $i$ produce intermediate goods according to the linear production function

$$
X_{t}^{i}=A_{t}^{i} E_{t}^{i}
$$

\footnotetext{
${ }^{5}$ Appendix A spells out households' value function and provides further details about the optimal migration decision.

${ }^{6}$ Notice that the aggregate marginal rate of substitution accounts for migration costs, i.e. it is adjusted to reflect the utility cost of labor market activities in each labor market following from (6).
} 
where employment is given as the sum of native and migrant workers in the region, $E_{t}^{i}=E_{t}^{i i}+E_{t}^{j i}$, and $A_{t}^{i}$ is an exogenous productivity process evolving according to

$$
\log \left(A_{t}^{i}\right)=\rho_{a} \log \left(A_{t-1}^{i}\right)+\nu_{a, t}^{i},
$$

where $\nu_{a, t}^{i}$ is an i.i.d. shock with zero mean and standard deviation $\sigma_{a}^{i}$. To hire new workers, firms post $V_{t}^{i i}$ vacancies for native workers and $V_{t}^{j i}$ vacancies for migrant workers at a cost of $\kappa$ per vacancy. The number of successful hires or matches is a function of searching workers and vacancies:

$$
M_{t}^{i i}=\tau_{t}^{i}\left(V_{t}^{i i}\right)^{\gamma}\left(S_{t}^{i i}\right)^{1-\gamma} ; M_{t}^{j i}=\tau_{t}^{i}\left(V_{t}^{j i}\right)^{\gamma}\left(S_{t}^{j i}\right)^{1-\gamma}
$$

where $1-\gamma$ captures the elasticity of matches to searchers, and matching efficiency evolves according to

$$
\log \left(\tau_{t}^{i}\right)=\left(1-\rho_{\tau}\right) \log \left(\tau^{i}\right)+\rho_{\tau} \log \left(\tau_{t-1}^{i}\right)+\nu_{\tau, t}^{i},
$$

where $\nu_{\tau, t}^{i}$ is an i.i.d. shock with zero mean and standard deviation $\sigma_{\tau}^{i}$. Labor market tightness in region $i$ is given by

$$
\theta_{t}^{i}=\theta_{t}^{i i} \frac{S_{t}^{i i}}{S_{t}^{i}}+\theta_{t}^{j i} \frac{S_{t}^{j i}}{S_{t}^{i}}
$$

where $S_{t}^{i}=S_{t}^{i i}+S_{t}^{j i}$ denote the total pool of searchers in labor market $i$ and where labor market tightness for native and migrant workers are defined, respectively, as $\theta_{t}^{i i}=V_{t}^{i i} / S_{t}^{i i}$ and $\theta_{t}^{j i}=V_{t}^{j i} / S_{t}^{j i}$. The probabilities of filling a vacancy of each kind are given by

$$
q_{t}^{i i}=\frac{M_{t}^{i i}}{V_{t}^{i i}}=\tau_{t}^{i}\left(\theta_{t}^{i i}\right)^{\gamma-1} \quad ; \quad q_{t}^{j i}=\frac{M_{t}^{j i}}{V_{t}^{j i}}=\tau_{t}^{i}\left(\theta_{t}^{j i}\right)^{\gamma-1} .
$$

By assumption, a filled vacancy becomes productive immediately. To derive the number of vacancies posted by intermediate firms, let $P_{x, t}^{i}$ denote the price of intermediate goods produced in region $i$. Since the intermediate firms operate under perfect competition, the marginal value of a filled vacancy of the two types of workers can be expressed in terms of the final consumption bundle ss $^{7}$

$$
\begin{aligned}
V_{J, t}^{i i} & =\frac{P_{x, t}^{i}}{P_{t}^{i}} A_{t}^{i}-\frac{W_{t}^{i i}}{P_{t}^{i}}+(1-\rho) \mathbf{E}_{\mathbf{t}}\left\{\mathcal{Q}_{t, t+1} V_{J, t+1}^{i i}\right\} \\
V_{J, t}^{j i} & =\frac{P_{x, t}^{i}}{P_{t}^{i}} A_{t}^{i}-\frac{W_{t}^{j i}}{P_{t}^{i}}+(1-\rho) \mathbf{E}_{\mathbf{t}}\left\{\mathcal{Q}_{t, t+1} V_{J, t+1}^{j i}\right\} .
\end{aligned}
$$

\footnotetext{
${ }^{7}$ The value of a match to the firm can be derived as the partial derivative of the value of the firm to its owners, in turn given as the present discounted value of profit streams, with respect to employment.
} 
The value of a filled vacancy to an intermediate producer is given by the marginal real revenue minus the real marginal cost (the real wage), plus the discounted continuation value from the match. With probability $(1-\rho)$, the job remains active and earns the expected value; the job is destroyed with probability $\rho$ and thus has zero value.

With free entry, the expected real value of posting a vacancy (e.g. $q_{t}^{i i} V_{J, t}^{i i}$ ) must equal the cost $\left(\kappa P_{H, t}^{i} / P_{t}^{i}\right)$. Hence, the marginal values of filled vacancies are constrained by the conditions

$$
V_{J, t}^{i i}=\frac{\kappa}{q_{t}^{i i}} \frac{P_{H, t}^{i}}{P_{t}^{i}} ; V_{J, t}^{j i}=\frac{\kappa}{q_{t}^{j i}} \frac{P_{H, t}^{i}}{P_{t}^{i}} .
$$

Combining equations (22) and (23) yields a job-creation condition determining the number of vacancies posted for each of the two types of workers:

$$
\begin{gathered}
\frac{\kappa}{q_{t}^{i i}} \frac{P_{H, t}^{i}}{P_{t}^{i}}=\frac{P_{x, t}^{i}}{P_{t}^{i}} A_{t}^{i}-\frac{W_{t}^{i i}}{P_{t}^{i}}+(1-\rho) \mathbf{E}_{\mathbf{t}}\left\{\mathcal{Q}_{t, t+1} \frac{\kappa}{q_{t+1}^{i i}} \frac{P_{H, t+1}^{i}}{P_{t+1}^{i}}\right\} \\
\frac{\kappa}{q_{t}^{j i}} \frac{P_{H, t}^{i}}{P_{t}^{i}}=\frac{P_{x, t}^{i}}{P_{t}^{i}} A_{t}^{i}-\frac{W_{t}^{j i}}{P_{t}^{i}}+(1-\rho) \mathbf{E}_{\mathbf{t}}\left\{\mathcal{Q}_{t, t+1} \frac{\kappa}{q_{t+1}^{j i}} \frac{P_{H, t+1}^{i}}{P_{t+1}^{i}}\right\} .
\end{gathered}
$$

\subsection{Wage determination}

Successful matches of searchers to vacancies generate an economic surplus given as the sum of the values of these matches to households and firms. For the employment of native workers in region $i$, we have $V_{J, t}^{i i}+V_{W, t}^{i i}$, where $V_{J, t}^{i i}$ is given by (22), and where the value of a native worker to household $i, V_{W, t}^{i i}$, reads ${ }^{8}$

$$
\begin{aligned}
V_{W, t}^{i i}= & \frac{W_{t}^{i i}}{P_{t}^{i}}-b^{U}-(1-\zeta) \frac{U_{l, t}^{i}}{U_{c, t}^{i}}\left[\frac{\left(1-\alpha_{1}\right)\left(1-l_{t}^{i}\right)}{E_{t}^{i i}+\zeta U_{t}^{i i}}\right]^{1 / \nu_{1}}+ \\
& (1-\rho) \mathbf{E}_{\mathbf{t}}\left\{\mathcal{Q}_{t, t+1}\left(1-f_{t+1}^{i i}\right) V_{W, t+1}^{i i}\right\} .
\end{aligned}
$$

By this expression, a match generates a surplus for the household in period $t$ by increasing real wage income at the expense of unemployment benefits and an additional disutility of working rather than being unemployed. In addition, with probability $(1-\rho)$, the match has an expected discounted continuation value increasing in the expected difficulty of finding a job in the next period.

Wages are determined through a Nash bargaining process to distribute the aggregate surplus between workers and firms. ${ }^{9}$ Specifically, the wage is chosen to maximize the

\footnotetext{
${ }^{8}$ The value of a match to the household can be derived as the partial derivative of the household's value function with respect to employment. See Appendix A for details.

${ }^{9}$ Some papers incorporating unemployment into New Keynesian models make specific assumptions that cause the wage-setting process to generate inefficient fluctuations; see e.g. Thomas (2008) or Blanchard and Galí (2010). We follow the baseline model by not imposing constraints on wage adjustments.
} 
Nash product $\left(V_{J, t}^{i i}\right)^{\eta_{t}}\left(V_{W, t}^{i i}\right)^{1-\eta_{t}}$, where $\eta_{t}$ denotes the stochastic bargaining power of firms, evolving as

$$
\log \left(\eta_{t}^{i}\right)=\left(1-\rho_{\eta}\right) \log (\eta)+\rho_{\eta} \log \left(\eta_{t-1}^{i}\right)+\nu_{\eta, t}^{i} .
$$

The real wage negotiated in this bargain, $W_{t}^{i i} / P_{t}^{i}$, satisfies the optimality condition

$$
\eta_{t}^{i} V_{W, t}^{i i}=\left(1-\eta_{t}^{i}\right) V_{J, t}^{i i}
$$

Combining the Nash bargaining rule with the the free-entry condition (23), the job-creation condition (25), and the definition of the marginal value of a native worker from equation (26) results in a wage equation for native workers in region $i$,

$$
\begin{aligned}
\frac{W_{t}^{i i}}{P_{t}^{i}} & =\eta_{t}^{i}\left[b^{U}+(1-\zeta) \frac{U_{l, t}^{i}}{U_{c, t}^{i}}\left[\frac{\left(1-\alpha_{1}\right)\left(1-l_{t}^{i}\right)}{E_{t}^{i i}+\zeta U_{t}^{i i}}\right]^{1 / \nu_{1}}\right] \\
& +\left(1-\eta_{t}^{i}\right)\left[\frac{P_{x, t}^{i}}{P_{t}^{i}} A_{t}^{i}+(1-\rho) \mathbf{E}_{\mathbf{t}}\left(\mathcal{Q}_{t, t+1} \kappa \theta_{t+1}^{i i} \frac{P_{H, t+1}^{i}}{P_{t+1}^{i}}\right)\right] .
\end{aligned}
$$

When firms have no bargaining power $\left(\eta_{t}^{i}=0\right.$ for all $\left.t\right)$, the wage is set to the upper bound of the wage bargaining set,

$$
\frac{W_{t}^{i i}}{P_{t}^{i}}=\frac{P_{x, t}^{i}}{P_{t}^{i}} A_{t}^{i}+(1-\rho) \mathbf{E}_{\mathbf{t}}\left(\mathcal{Q}_{t, t+1} \kappa \theta_{t+1}^{i i} \frac{P_{H, t+1}^{i}}{P_{t+1}^{i}}\right),
$$

and households earn their full marginal product in addition to a premium increasing in expected future labor market tightness. When households have no bargaining power, $\left(\eta_{t}^{i}=1\right.$ for all $\left.t\right)$, the wage is set at the lower bound of the bargaining set:

$$
\frac{W_{t}^{i i}}{P_{t}^{i}}=b^{U}+(1-\zeta) \frac{U_{l, t}^{i}}{U_{c, t}^{i}}\left[\frac{\left(1-\alpha_{1}\right)\left(1-l_{t}^{i}\right)}{E_{t}^{i i}+\zeta U_{t}^{i i}}\right]^{1 / \nu_{1}}
$$

In this case, workers have to accept a wage equal to the value of their time when participating in this particular labor market in addition to the unemployment benefit, i.e. their reservation wage. In intermediate cases, the surplus is split between workers and firms according to their respective bargaining power. Similarly, the wage equation for migrant workers in $i$ states as

$$
\begin{aligned}
\frac{W_{t}^{j i}}{P_{t}^{i}}= & {\left[\frac{\eta_{t}^{i}}{\eta_{t}^{i}+Q_{t}^{i}\left(1-\eta_{t}^{i}\right)}\right]\left[b^{U}+Q_{t}^{i}(1-\zeta) \frac{U_{l, t}^{j}}{U_{c, t}^{j}}\left[\frac{\alpha_{1}\left(1-l_{t}^{j}\right)}{E_{t}^{j i}+\zeta U_{t}^{j i}}\right]^{1 / \nu_{1}}\right] } \\
+ & {\left[\frac{Q_{t}^{i}\left(1-\eta_{t}^{i}\right)}{\eta_{t}^{i}+Q_{t}^{i}\left(1-\eta_{t}^{i}\right)}\right]\left[\frac{P_{x, t}^{i}}{P_{t}^{i}} A_{t}^{i}\right]+\left[\frac{Q_{t}^{i}\left(1-\eta_{t}^{i}\right)}{\eta_{t}^{i}+Q_{t}^{i}\left(1-\eta_{t}^{i}\right)}\right](1-\rho) } \\
& \mathbf{E}_{\mathbf{t}}\left\{\mathcal{Q}_{t, t+1} \frac{\kappa}{q_{t+1}^{j i}} \frac{P_{H, t+1}^{i}}{P_{t+1}^{i}}\left(1-\frac{Q_{t+1}^{i}}{Q_{t}^{i}}\left(1-f_{t+1}^{j i}\right)\right)\right\}
\end{aligned}
$$




\subsection{Final goods producers}

Final goods producers are retailers combining domestically produced intermediate goods into a final consumption good sold in region $i$ and exported to region $j$. Any firm $l \in[0,1]$ produces a differentiated good using the common technology

$$
Y_{t}^{i}(l)=\left(X_{t}^{i}(l)\right)^{1-\alpha}
$$

in a regime of monopolistic competition. Aggregate production of final goods reads

$$
Y_{t}^{i} \equiv\left[\int_{0}^{1}\left(Y_{t}^{i}(l)\right)^{\frac{\varepsilon-1}{\varepsilon}} d l\right]^{\frac{\varepsilon}{\varepsilon-1}}=\left(\frac{X_{t}^{i}}{\Delta_{H, t}^{i}}\right)^{1-\alpha},
$$

where

$$
\Delta_{H, t}^{i} \equiv \int_{0}^{1}\left[\frac{P_{H, t}^{i}(l)}{P_{H, t}^{i}}\right]^{\frac{-\varepsilon}{1-\alpha}} d l
$$

is a measure of price dispersion, and the price index is defined as

$$
P_{H, t}^{i}=\left[\int_{0}^{1} P_{H, t}^{i}(l)^{1-\varepsilon} d l\right]^{\frac{1}{1-\varepsilon}} .
$$

The real marginal cost of production, defined in terms of domestic prices, $P_{H, t}^{i}$, becomes

$$
R M C_{t}^{i}(l)=\frac{M C_{t}^{i}(l)}{P_{H, t}^{i}}=\frac{P_{x, t}^{i}}{P_{t}^{i}} \frac{P_{t}^{i}}{P_{H, t}^{i}} \frac{\left[Y_{t}^{i}(l)\right]^{\frac{\alpha}{1-\alpha}}}{1-\alpha} .
$$

Final retailers face a downward-sloping demand function, capturing consumption demand from households in both regions $i$ and $j$ as well as demand from intermediate producers in $i$, whose vacancy cost is denominated in the final consumption good

$$
\begin{aligned}
Y_{t}^{i}(l) & =\left(\frac{P_{H, t}^{i}(l)}{P_{H, t}^{i}}\right)^{-\varepsilon}\left[C_{H, t}^{i}+C_{F, t}^{j}+\kappa V_{t}^{i}\right] \\
& =\left(\frac{P_{H, t}^{i}(l)}{P_{H, t}^{i}}\right)^{-\varepsilon}\left[\left(1-\alpha_{2}\right)\left(\frac{P_{H, t}^{i}}{P_{t}^{i}}\right)^{-\nu_{2}} C_{t}^{i}+\alpha_{2}\left(\frac{P_{F, t}^{j}}{P_{t}^{j}}\right)^{-\nu_{2}} C_{t}^{j}+\kappa V_{t}^{i}\right],
\end{aligned}
$$

where market clearing requires that $Y_{t}^{i}=C_{H, t}^{i}+C_{F, t}^{j}+\kappa V_{t}^{i}$. Prices in the final sector are assumed to be sticky as in Calvo (1983). In any period, each firm has the chance to reset its price with probability $1-\delta$ so as to maximize the expected discounted stream of future profits. With probability $\delta$, the firm sticks to the price charged in the previous period. That is, selected firms set a new price $P_{H, t}^{i \star}(l)$ to maximize

$$
\mathbf{E}_{\mathbf{t}} \sum_{s=0}^{\infty}(\delta)^{s} \mathcal{Q}_{t, t+s}\left[(1-\varsigma) P_{H, t}^{i \star}(l) Y_{t+s}^{i}(l)-P_{x, t+1}^{i}(l) X_{t+1}^{i}(l)\right],
$$


where $\varsigma>0$ is a production subsidy, subject to production (33) and demand (38). Since all firms that can change prices set the same price so that $P_{H, t}^{i}(l)=P_{H, t}^{i \star} \forall l$, the first-order condition becomes

$$
\mathbf{E}_{\mathbf{t}} \sum_{s=0}^{\infty}(\delta)^{s} \mathcal{Q}_{t, t+s} Y_{t+s}\left[P_{H, t}^{i \star}-\frac{1}{1-\varsigma} \frac{\varepsilon}{\varepsilon-1} M C_{t+s}^{i}(l)\right]=0 .
$$

This condition reflects the forward-looking nature of price-setting: with nominal rigidities, monopolistic competitive firms set prices as a mark-up over marginal costs in expected discounted terms. Using (38), and noting that $\mathcal{Q}_{t, t+s}=\beta^{s} U_{t+s}^{i} P_{t}^{i} / U_{t}^{i} P_{t+s}^{i}$, the first-order condition can be rewritten in terms of aggregate variables in the recursive form

$$
\frac{P_{H, t}^{i \star}}{P_{H, t}^{i}}=\left(\frac{F_{1, t}^{i}}{F_{2, t}^{i}}\right)^{\frac{1-\alpha}{1-\alpha+\varepsilon \alpha}}
$$

where

$$
F_{1, t}^{i} \equiv \frac{1}{1-\varsigma} \frac{\varepsilon}{\varepsilon-1} U_{c, t}^{i} R M C_{t}^{i} \frac{P_{H, t}^{i}}{P_{t}^{i}} Y_{t}^{i}+\delta \beta \mathbf{E}_{t}\left\{\left(\Pi_{H, t+1}^{i}\right)^{\frac{\varepsilon}{1-\alpha}} F_{1, t+1}^{i}\right\}
$$

represents the present discounted value of cost, and

$$
F_{2, t}^{i} \equiv U_{c, t}^{i} \frac{P_{H, t}^{i}}{P_{t}^{i}} Y_{t}^{i}+\delta \beta \mathbf{E}_{t}\left\{\left(\Pi_{H, t+1}^{i}\right)^{\varepsilon-1} F_{2, t+1}^{i}\right\}
$$

represents the present discounted value of revenues. In turn, from the definition of the price index, the reset price is related to domestic inflation so that

$$
\left(\frac{1-\delta\left(\Pi_{H, t}^{i}\right)^{\varepsilon-1}}{1-\delta}\right)^{\frac{1}{1-\varepsilon}}=\frac{P_{H, t}^{i \star}}{P_{H, t}^{i}}=\left(\frac{F_{1, t}^{i}}{F_{2, t}^{i}}\right)^{\frac{1-\alpha}{1-\alpha+\varepsilon \alpha}} .
$$

Finally, with Calvo price-setting, the price index implies that the measure of price dispersion evolves according to

$$
\Delta_{t}^{i}=\delta\left(\Pi_{H, t}^{i}\right)^{\frac{\varepsilon}{1-\alpha}} \Delta_{t-1}^{i}+(1-\delta)\left(\frac{1-\delta\left(\Pi_{H, t}^{i}\right)^{\varepsilon-1}}{1-\delta}\right)^{\frac{\varepsilon}{(1-\varepsilon)(\alpha-1)}}
$$

\subsection{Market clearing}

Aggregate output in the retail sector in region $i$ is defined as

$$
Y_{t}^{i} \equiv\left[\int_{0}^{1} Y_{t}^{i}(l)^{\frac{\varepsilon-1}{\varepsilon}} d l\right]^{\frac{\varepsilon}{\varepsilon-1}}
$$


such that integrating the demand for good $l$, given by (38), yields a conventional aggregate resource constraint:

$$
Y_{t}^{i}=\left[\left(1-\alpha_{2}\right)+\alpha_{2} \frac{\left(1-\alpha_{2}\right)\left(Q_{t}^{i}\right)^{1-\nu_{2}}-\alpha_{2}}{\left(1-\alpha_{2}\right)-\alpha_{2}\left(Q_{t}^{i}\right)^{1-\nu_{2}}}\right]^{\frac{\nu_{2}}{1-\nu_{2}}}\left[\left(1-\alpha_{2}\right) C_{t}^{i}+\alpha_{2}\left(Q_{t}^{i}\right)^{\nu_{2}} C_{t}^{j}\right]+\kappa V_{t}^{i}
$$

The aggregate production function results from combining the demand for final goods (38) with the respective production function (33),

$$
Y_{t}^{i}=\left(X_{t}^{i}\right)^{1-\alpha}\left(\Delta_{t}^{i}\right)^{\alpha-1}
$$

Finally, aggregate (un)employment, vacancies, and matches are defined as follows:

$$
\begin{aligned}
& E_{t}^{i}=E_{t}^{i i}+E_{t}^{j i} ; U_{t}^{i}=U_{t}^{i i}+U_{t}^{j i} ; V_{t}^{i}=V_{t}^{i i}+V_{t}^{j i} ; M_{t}^{i}=M_{t}^{i i}+M_{t}^{j i} \\
& E_{t}^{j}=E_{t}^{j j}+E_{t}^{i j} ; U_{t}^{j}=U_{t}^{j j}+U_{t}^{i j} ; V_{t}^{j}=V_{t}^{j j}+V_{t}^{i j} ; M_{t}^{j}=M_{t}^{j j}+M_{t}^{i j} .
\end{aligned}
$$

\subsection{Monetary policy}

A specification of monetary policy is needed to close the model. ${ }^{10}$ For comparison with the optimal monetary policy regime described below, we sometimes let the common monetary authority follow versions of a simple interest rate rule taking the log-linear form

$$
\hat{r}_{t}=\rho_{r} \hat{r}_{t-1}+\left(1-\rho_{r}\right)\left(\Gamma_{\pi} \hat{\pi}_{t}+\Gamma_{u} \hat{u}_{t}+\Gamma_{y} \hat{y}_{t}\right),
$$

where $\hat{x}_{t} \equiv \log \left(X_{t}\right)-\log (X)$ denotes $\log$ deviations of a generic variable $X_{t}$ from its steady-state value. The parameter $\rho_{r} \in[0,1)$ denotes the degree of interest rate smoothing, and $\Gamma_{\pi}>1, \Gamma_{u}$, and $\Gamma_{y}$ represent the weights on union-wide inflation $\left(\hat{\pi}_{t}=\hat{\pi}_{H, t}^{i} / 2+\hat{\pi}_{H, t}^{j} / 2\right)$, unemployment $\left(\hat{u}_{t}=\hat{u}_{t}^{i} / 2+\hat{u}_{t}^{j} / 2\right)$, and output $\left(\hat{y}_{t}=\hat{y}_{t}^{i} / 2+\hat{y}_{t}^{j} / 2\right)$, respectively.

\section{Efficient and natural equilibrium}

The efficient and natural allocations are two important reference points in the model. The efficient equilibrium is defined as the solution to a social planner's problem. The natural equilibrium is the market outcome for the special case in which prices are fully flexible. We consider these two special cases in turn.

\footnotetext{
${ }^{10}$ For the full set of equilibrium conditions, see Appendix C.
} 


\subsection{Efficient allocation}

A cooperative social planner chooses a real allocation to maximize welfare measured by the sum of the representative utility functions in the two regions of the monetary union,

$$
\mathbf{E}_{\mathbf{0}} \sum_{t=0}^{\infty} \beta^{t}\left(\mathcal{U}_{t}^{i}+\mathcal{U}_{t}^{j}\right)
$$

subject to technology, matching, and resource constraints (2)-(4), (6)-(8), (15), (17), (33), and the aggregate resource constraint $Y_{t}^{i}=C_{H, t}^{i}+C_{F, t}^{j}+\kappa V_{t}^{i}$ for region $i$, as well as the corresponding relations for region $j .{ }^{11}$

As shown in Appendix D, the first-order conditions can be reduced to a set of efficiency conditions for labor market outcomes. In particular, efficiency in the market for domestic employment in region $i$ demands

$$
\begin{gathered}
(1-\zeta) \frac{U_{l, t}^{i}}{U_{c, t}^{i}} \frac{\left[\left(1-\alpha_{1}\right)\left(1-l_{t}^{i}\right) /\left(E_{t}^{i i}+\zeta U_{t}^{i i}\right)\right]^{1 / \nu_{1}}}{\left[\left(1-\alpha_{2}\right) C_{t}^{i} / C_{H, t}^{i}\right]^{1 / \nu_{2}}}=\frac{(1-\alpha) Y_{t}^{i}}{E_{t}^{i}}-\frac{\kappa}{\gamma q_{t}^{i i}} \\
+\beta(1-\rho) \mathbf{E}_{\mathbf{t}}\left\{\frac{U_{c, t+1}^{i}}{U_{c, t}^{i}} \frac{\left[\left(1-\alpha_{2}\right) C_{t+1}^{i} / C_{H, t+1}^{i}\right]^{1 / \nu_{2}}}{\left[\left(1-\alpha_{2}\right) C_{t}^{i} / C_{H, t}^{i}\right]^{1 / \nu_{2}}}\left[1-(1-\gamma) \kappa f_{t+1}^{i i}\right] \frac{\kappa}{\gamma q_{t+1}^{i i}}\right\} .
\end{gathered}
$$

Corresponding optimality conditions hold for the markets for domestic labor in region $j$, and for migrant labor in $i$ as well as $j .^{12}$ As in the standard model with a Walrasian labor market, the social planner increases employment up until the point where the opportunity cost of consumption in terms of leisure equals the marginal rate of substitution between them. The left-hand side of (50) represents the marginal rate of substitution. It is appropriately corrected to reflect the specific utility cost associated with additional labor market activities in the market for native workers in region $i$ as well as the specific utility gain from the associated increase and composition of consumption for region $i$ households. The right-hand side represents the marginal rate of transformation. The first term is the marginal product of labor, which fully captures the opportunity cost in standard closed-economy models with Walrasian labor markets. Matching frictions give rise to two additional components. On the one hand, the additional output available for consumption is reduced by the marginal cost of matching incurred to bring the increase in employment about. This effect is captured by the second term. On the other hand, as a fraction of matches continues into the

\footnotetext{
${ }^{11}$ Of course, welfare in the economy would be higher if the allocation of workers to firms were frictionless. By including the matching technology as a constraint, the solution to the social planner's problem establishes the constrained efficient allocation; see e.g. Greenwald and Stiglitz (1986). Using this allocation as a reference point for optimal monetary policy avoids introducing an additional source of a systematic inflation bias in monetary policy.

${ }^{12}$ Blanchard and Galí (2010) and Thomas (2008) derive similar conditions for the closed economy.
} 
next period, an increase in employment in this period reduces marginal hiring costs in the next. The third forward-looking term captures the present discounted value of these savings, where the future marginal matching cost per unit has been corrected to reflect the expected change in labor market conditions.

In the benchmark model without mobility, regional conditions corresponding to (50) are sufficient to characterize the efficient allocation. In particular, since the mass of searchers in each labor market is pre-determined, an optimal choice of employment in each labor market uniquely pins down the required number of vacancies and so the efficient level of labor market tightness. In the full model with a mobile labor force, however, the social planner can simultaneously reallocate searchers across labor markets. Labor mobility therefore gives rise to an additional efficiency condition for region $i$ workers, given as

$$
\frac{U_{l, t}^{i}}{U_{c, t}^{i}} \zeta\left\{\left[\frac{\alpha_{1}\left(1-l_{t}^{i}\right)}{E_{t}^{i j}+\zeta U_{t}^{i j}}\right]^{\frac{1}{\nu_{1}}}-\left[\frac{\left(1-\alpha_{1}\right)\left(1-l_{t}^{i}\right)}{E_{t}^{i i}+\zeta U_{t}^{i i}}\right]^{\frac{1}{\nu_{1}}}\right\}=\frac{\gamma-1}{\gamma} \kappa\left\{\left[\frac{\left(1-\alpha_{2}\right) C_{t}^{i}}{C_{H, t}^{i}}\right]^{\frac{1}{\nu_{2}}} \theta_{t}^{i i}-\left[\frac{\alpha_{2} C_{t}^{i}}{C_{F, t}^{i}}\right]^{\frac{1}{\nu_{2}}} \theta_{t}^{i j}\right\} .
$$

A corresponding condition applies for workers from region $j$. According to condition (51), the social planner allocates workers across labor markets up until the point where the relative marginal vacancy cost (on the right-hand side) equals the relative disutilities of search activities in the two labor markets (on the left-hand side). In the special case where labor market activities are costless to the unemployed so that $\zeta=0$, the social planner allocates searchers so as to equalize marginal vacancy costs in regional labor markets.

\subsection{Sources of inefficiencies and natural allocation}

To fix ideas, let us first consider the benchmark model without labor mobility. ${ }^{13}$ Three are the sources of inefficiencies, when assuming away unemployment benefits (so that $b^{U}=0$ ): First, the distortion from monopolistic competition, which is eliminated by an appropriate production subsidy. This implies that the average markup in the final goods sector, $\mu_{t}^{i} \equiv(1-\alpha)\left(Y_{t}^{i}\right)^{\alpha /(1-\alpha)} P_{x, t}^{i} / P_{t}^{i}$, and price dispersion, $\Delta_{t}^{i}$, are equal to one. Second, market distortions related to search and matching frictions in the labor market are eliminated with the Hosios (1990) condition. Specifically, when a firm posts a new vacancy, it has a negative congestion externality for other firms (because competition for workers to fill vacancies has increased) but a positive thick-market externality for workers (because competition for jobs has decreased). When $\eta=\gamma$, the private marginal value of an additional vacancy coincides with its social value and the two externalities perfectly offset each other in the market equilibrium. The third and remaining source of inefficiency are nominal price rigidities. Consequentially, both the zero-inflation steady state and the natural, flexible-price equilibrium are efficient.

All three sources of inefficiencies are also present in the full model with a mobile labor force. Hence, assuming away unemployment benefits, the job creation condition

\footnotetext{
${ }^{13}$ Appendix B provides all equilibrium conditions for the benchmark model with a fixed labor force.
} 
(25) and the wage equation (29) can be combined to give the flexible-price natural equilibrium condition for the market for domestic labor in region $i$ :

$$
\begin{gathered}
(1-\zeta) \frac{U_{l, t}^{i}}{U_{c, t}^{i}} \frac{\left[\left(1-\alpha_{1}\right)\left(1-l_{t}^{i}\right) /\left(E_{t}^{i i}+\zeta U_{t}^{i i}\right)\right]^{1 / \nu_{1}}}{\left[\left(1-\alpha_{2}\right) C_{t}^{i} / C_{H, t}^{i}\right]^{1 / \nu_{2}}}=\frac{(1-\alpha) Y_{t}^{i}}{E_{t}^{i}}-\frac{\kappa}{\eta_{t}^{i} q_{t}^{i i}} \\
+\beta(1-\rho) \mathbf{E}_{\mathbf{t}}\left\{\frac{U_{c, t+1}^{i}}{U_{c, t}^{i}} \frac{\left[\left(1-\alpha_{2}\right) C_{t+1}^{i} / C_{H, t+1}^{i}\right]^{1 / \nu_{2}}}{\left[\left(1-\alpha_{2}\right) C_{t}^{i} / C_{H, t}^{i}\right]^{1 / \nu_{2}}}\left[1-\left(1-\eta_{t}^{i}\right) \kappa f_{t+1}^{i i}\right] \frac{\kappa}{\eta_{t}^{i} q_{t+1}^{i i}}\right\} .
\end{gathered}
$$

The optimal migration decision (14) can be rewritten as

$$
\begin{aligned}
& \frac{U_{l, t}^{i}}{U_{c, t}^{i}} \zeta\left\{\left[\frac{\alpha_{1}\left(1-l_{t}^{i}\right)}{E_{t}^{i j}+\zeta U_{t}^{i j}}\right]^{\frac{1}{\nu_{1}}}-\left[\frac{\left(1-\alpha_{1}\right)\left(1-l_{t}^{i}\right)}{E_{t}^{i i}+\zeta U_{t}^{i i}}\right]^{\frac{1}{\nu_{1}}}\right\} \\
& =\frac{\gamma-1}{\gamma} \kappa\left\{\left[\frac{\left(1-\alpha_{2}\right) C_{t}^{i}}{C_{H, t}^{i}}\right]^{\frac{1}{\nu_{2}}} \theta_{t}^{i i}-\left[\frac{\alpha_{2} C_{t}^{i}}{C_{F, t}^{i}}\right]^{\frac{1}{\nu_{2}}} \theta_{t}^{i j}\right\} \\
& -\beta(1-\rho) \mathbf{E}_{\mathbf{t}}\left\{\frac{U_{c, t+1}^{i}}{U_{c, t}^{i}} \frac{1-\eta_{t}^{i}}{\eta_{t}^{i}}\left[f_{t}^{i i}\left(1-f_{t+1}^{i i}\right) V_{J, t+1}^{i i}-f_{t}^{i j}\left(1-f_{t+1}^{i j}\right) V_{J, t+1}^{i j}\right]\right\} .
\end{aligned}
$$

The natural labor market equilibrium (52) coincides with (50) in the absence of bargainingpower shocks (i.e. $\eta_{t}^{i}=\eta$ for all $t$ ) and whenever the Hosios (1990) condition $\eta=\gamma$ holds. When labor is mobile, however, the Hosios condition is not sufficient to render the flexible-price equilibrium efficient out of steady state. In particular, while the Hosios condition is sufficient to equalize the private and social marginal value of vacancies, $\eta=\gamma$ does not ensure that the optimal migration decision in the market equilibrium (53) coincides with the efficiency condition (51). Effectively, unless all employment contracts are terminated at the end of every period so that $\rho=0$ (or households fully discount the future so that $\beta=0$ ), households take too strong a signal from expected future labor market outcomes when making their migration decisions. In the full model, therefore, standard distortions from nominal price rigidities interact only with the remaining distortion from inefficient migration decisions in the market equilibrium.

Throughout, we assume that the Hosios condition holds, that a production subsidy offsets the distortion from monopolistic competition, and, unless otherwise stated, that unemployment benefits are unavailable and shocks to bargaining power are absent.

\section{Optimal monetary policy}

Under optimal monetary policy, the monetary authority is assumed to maximize welfare across the monetary union subject to the behavior of households and firms in the economy's market equilibrium. By contrast to the social planner, optimizing 
policymakers cannot sidestep any of the frictions that are associated with the interaction of agents in markets, in particular nominal rigidities and inefficient migration decisions. We characterize the optimal policy plan within the linear-quadratic framework proposed by Rotemberg and Woodford (1999). We first derive a welfare loss function from a second-order approximation of the sum of households' utility losses associated with deviations of consumption and leisure from the efficient allocation. We then minimize this loss function subject to linear approximations of the economy's market equilibrium conditions around the efficient steady state. Throughout, we focus on the solution under commitment from a timeless perspective, as in Woodford (1999).

\subsection{Loss function}

A second-order approximation of welfare (49) can be written as the expected present value of period losses

$$
W \simeq-\frac{1}{2} \mathbf{E}_{\mathbf{0}} \sum_{t=0}^{\infty} \beta^{t} L_{t}^{i}
$$

for $i \in[B M, L M]$, where $B M$ refers to the benchmark model with a fixed labor force and where $L M$ refers to the full model with labor mobility. As we show below, the period loss functions, $L_{t}^{i}$, comprise terms of variables in log deviation from their respective efficient levels. That is, for a generic variable, $X_{t}$, the loss function is expressed in terms of $\tilde{x}_{t} \equiv \log \left(X_{t}\right)-\log \left(X_{t}^{\star}\right)$. For future reference, note that $\tilde{x}_{t} \equiv$ $\hat{x}_{t}-\hat{x}_{t}^{*}$, where $\hat{x}_{t} \equiv \log \left(X_{t}\right)-\log (X)$ denotes the log deviation from the efficient steady state. For the benchmark model without labor mobility, the period loss function states as

$$
\begin{aligned}
L_{t}^{B M}= & \Gamma_{\pi}\left[\left(\tilde{\pi}_{H, t}^{i}\right)^{2}+\left(\tilde{\pi}_{H, t}^{j}\right)^{2}\right]+\Gamma_{l}\left[\left(\tilde{l}_{t}^{i}\right)^{2}+\left(\tilde{l}_{t}^{j}\right)^{2}\right]+ \\
& \Gamma_{c}\left[\left(\tilde{c}_{t}^{i}\right)^{2}+\left(\tilde{c}_{t}^{j}\right)^{2}\right]+\Gamma_{c_{H}}\left[\left(\tilde{c}_{H, t}^{i}\right)^{2}+\left(\tilde{c}_{H, t}^{j}\right)^{2}\right]+\Gamma_{c_{F}}\left[\left(\tilde{c}_{F, t}^{i}\right)^{2}+\left(\tilde{c}_{F, t}^{j}\right)^{2}\right]+ \\
& \Gamma_{e}\left[\left(\tilde{e}_{t}^{i}\right)^{2}+\left(\tilde{e}_{t}^{j}\right)^{2}\right]+\Gamma_{\theta}\left[\left(\tilde{\theta}_{t}^{i}\right)^{2}+\left(\tilde{\theta}_{t}^{j}\right)^{2}\right]+t . i . p .
\end{aligned}
$$

with an appropriately defined set of composite parameters, $\Gamma$, and terms independent of policy that may be safely ignored for monetary policy purposes collected in t.i.p. (see Appendix $\mathrm{F}$ for details). Like a conventional loss function derived from the canonical New Keynesian model, (55) contains quadratic terms in inflation and measures of real activity in deviation from efficient levels. Because inflation causes price dispersion and an inefficient distribution of demand across firms, price stability is a key goal for monetary policy. As in Benigno (2004), the union's common monetary policy authority seeks to stabilize an average of regional producer price inflation rates. Contrary to Benigno (2004), who assumes unitary trade elasticity, the terms of trade gap is not a sufficient statistic for the efficiency gaps in the interregional allocation of aggregate 
demand across firms. With our more general consumption aggregator, these gaps are expressed directly in terms of efficiency gaps in the consumption of home and foreign goods. Similarly, with search and matching frictions in labor markets, the welfare-relevant deviations of aggregate consumption and leisure do not reduce to a simple summary measure of real activity such as the output gaps appearing in the loss functions of simpler models with competitive labor market (see e.g. Thomas, 2008). Instead, labor market frictions give rise to additional terms in employment and labor market tightness (similar to Ravenna and Walsh, 2011). In the monetary union, moreover, the common policymaker responds to averages of regional labor market variables.

The period loss function for our full model with labor mobility is given as

$$
\begin{aligned}
L_{t}^{L M}= & \Gamma_{\pi}\left[\left(\tilde{\pi}_{H, t}^{i}\right)^{2}+\left(\tilde{\pi}_{H, t}^{j}\right)^{2}\right]+\Gamma_{l}\left[\left(\tilde{l}_{t}^{i}\right)^{2}+\left(\tilde{l}_{t}^{j}\right)^{2}\right]+ \\
& \Gamma_{c}\left[\left(\tilde{c}_{t}^{i}\right)^{2}+\left(\tilde{c}_{t}^{j}\right)^{2}\right]+\Gamma_{c_{H}}\left[\left(\tilde{c}_{H, t}^{i}\right)^{2}+\left(\tilde{c}_{H, t}^{j}\right)^{2}\right]+\Gamma_{c_{F}}\left[\left(\tilde{c}_{F, t}^{i}\right)^{2}+\left(\tilde{c}_{F, t}^{j}\right)^{2}\right]+ \\
& \Gamma_{e}^{i i}\left[\left(\tilde{e}_{t}^{i i}\right)^{2}+\left(\tilde{e}_{t}^{j j}\right)^{2}\right]+\Gamma_{e}^{i j}\left[\left(\tilde{e}_{t}^{i j}\right)^{2}+\left(\tilde{e}_{t}^{j i}\right)^{2}\right]+ \\
& \Gamma_{\theta^{i i}}\left[\left(\tilde{\theta}_{t}^{i i}\right)^{2}+\left(\tilde{\theta}_{t}^{j j}\right)^{2}\right]+\Gamma_{\theta^{i j}}\left[\left(\tilde{\theta}_{t}^{i j}\right)^{2}+\left(\tilde{\theta}_{t}^{j i}\right)^{2}\right]+ \\
& \Gamma_{\theta e}\left\{\left(1-\alpha_{1}\right)\left(\tilde{\theta}_{t}^{i i} \tilde{e}_{t}^{i i}+\tilde{\theta}_{t}^{j j} \tilde{e}_{t}^{j j}\right)+\alpha_{1}\left(\tilde{\theta}_{t}^{i j} \tilde{e}_{t}^{i j}+\tilde{\theta}_{t}^{j i} \tilde{e}_{t}^{j i}\right)+\right. \\
& \left(1-\alpha_{1}\right) \frac{\zeta \rho(1-f)}{f}\left[\tilde{\omega}_{t}^{i}\left(\tilde{\theta}_{t}^{i i}-\tilde{\theta}_{t}^{i j}\right)+\tilde{\omega}_{t}^{j}\left(\tilde{\theta}_{t}^{j j}-\tilde{\theta}_{t}^{j i}\right)\right]- \\
& \zeta(1-\rho)(1-f)\left[\left(1-\alpha_{1}\right)^{2}\left(\tilde{\theta}_{t}^{i i} \tilde{e}_{t-1}^{i i}+\tilde{\theta}_{t}^{j j} \tilde{e}_{t-1}^{j j}\right)+\alpha_{1}^{2}\left(\tilde{\theta}_{t}^{i i} \tilde{e}_{t-1}^{i j}+\tilde{\theta}_{t}^{j j} \tilde{e}_{t-1}^{j i}\right)\right]- \\
& \left.\zeta(1-\rho)(1-f)\left[\alpha_{1}\left(1-\alpha_{1}\right)\left(\tilde{\theta}_{t}^{i j} \tilde{e}_{t-1}^{i i}+\tilde{\theta}_{t}^{j i} \tilde{e}_{t-1}^{j j}+\tilde{\theta}_{t}^{i j} \tilde{e}_{t-1}^{i j}+\tilde{\theta}_{t}^{j i} \tilde{e}_{t-1}^{j i}\right)\right]\right\}+ \\
& \Gamma_{e^{i i} e^{j i}}\left(\tilde{e}_{t}^{i i} \tilde{e}_{t}^{j i}+\tilde{e}_{t}^{j j} \tilde{e}_{t}^{i j}\right)+t . i \cdot p .,
\end{aligned}
$$

again for an appropriately defined set of composite parameters, $\Gamma$, and with terms independent of policy collected in t.i.p. (see Appendix G for details). As in the benchmark model, the loss function contains quadratic terms in regional inflation, leisure, and consumption (aggregate as well as its composition of home and foreign goods). With labor mobility, however, losses no longer depend simply on fluctuations in aggregate region-specific levels of employment and labor market tightness. Instead, the loss function for the full model contains separate quadratic terms for these labor market variables for native and migrant workers in each region. The disaggregated terms stem from the assumption that the household utility function is characterized by a positive and non-decreasing marginal disutility of labor market activities for native and migrant workers, respectively. Finally, labor mobility gives rise to a set of unconventional cross-product terms penalizing inefficient co-variation in labor market tightness and search activity across labor markets as well as home and migrant employment. 


\subsection{Constraints and optimality conditions}

A key constraint for monetary policy is the New Keynesian Phillips curve for producer price inflation resulting from a log-linearization of the price-setting conditions in (39)-(43). As we show in Appendix G, the Phillips curve can be written as

$$
\tilde{\pi}_{H, t}^{i}=\beta \mathbf{E}_{t} \tilde{\pi}_{H, t+1}^{i}+\lambda \widetilde{r m} c_{t}^{i} .
$$

Since the Hosios condition ensures that the labor market equilibrium is efficient, the monetary authority does not face any conventional trade-off-inducing cost-push disturbances in the sense that any policy that stabilizes real marginal cost in region $i$ delivers regional price stability.

Subject to region-specific disturbances, however, the common monetary policy authority cannot achieve price stability in both regions simultaneously with its single union-wide instrument. And even if it could, as in Benigno (2004), policymakers face a trade-off between price stability in individual regions and the appropriate allocation of demand across the monetary union. Specifically, relative price inflation must satisfy

$$
\tilde{\pi}_{H, t}^{i}-\tilde{\pi}_{H, t}^{j}=\frac{\tilde{\mathrm{q}}_{t}-\tilde{\mathrm{q}}_{t-1}+\hat{\mathrm{q}}_{t}^{*}-\hat{\mathrm{q}}_{t-1}^{*}}{1-2 \alpha_{2}}
$$

where $\hat{\mathrm{q}}_{t}^{*}$ is the real exchange rate that can support the efficient equilibrium. Whenever a region-specific shock calls for an adjustment in relative prices, as reflected in movements in $\hat{\mathrm{q}}_{t}^{*}$, monetary policy must balance the expenditure switching that follows from its policy stance through actual movements in the real exchange rate against the relative cost of inflation across regions.

Moreover, with inefficient migration decisions, region-specific disturbances are introducing trade-off-inducing movements in employment and labor market tightness across the union. To see this, note that the policymaker optimizes subject also to the migration behavior, which for households in region $i$ can be expressed as

$$
\begin{aligned}
& \Lambda_{1}\left(\tilde{e}_{t}^{i i}-\tilde{e}_{t}^{i j}\right)+\Lambda_{2}\left(\tilde{e}_{t-1}^{i i}-\tilde{e}_{t-1}^{i j}\right)+\Lambda_{3}\left(\tilde{\theta}_{t}^{i i}-\tilde{\theta}_{t}^{i j}\right)+\Lambda_{4} \mathbf{E}_{t}\left(\tilde{\theta}_{t+1}^{i i}-\tilde{\theta}_{t+1}^{i j}\right)+ \\
& \Lambda_{5}\left(\tilde{c}_{t}^{i}-\tilde{c}_{H, t}^{i}-\tilde{c}_{t}^{j}+\tilde{c}_{H, t}^{j}\right)+\Lambda_{6} \mathbf{E}_{t}\left(\tilde{c}_{t+1}^{i}-\tilde{c}_{H, t+1}^{i}-\tilde{c}_{t+1}^{j}+\tilde{c}_{H, t+1}^{j}\right)+ \\
& \Lambda_{7}\left(\hat{\theta}_{t}^{i i \star}-\hat{\theta}_{t}^{i j \star}\right)+\Lambda_{8} \mathbf{E}_{t}\left(\hat{\theta}_{t+1}^{i i \star}-\hat{\theta}_{t+1}^{i j \star}\right)+\Lambda_{9} \mathbf{E}_{t}\left(\hat{c}_{t+1}^{i \star}-\hat{c}_{H, t+1}^{i \star}-\hat{c}_{t+1}^{j \star}+\hat{c}_{H, t+1}^{j \star}\right)+ \\
& \Lambda_{10}\left(\tilde{\mathrm{q}}_{t}+\hat{\mathrm{q}}_{t}^{\star}\right)+\Lambda_{11} \mathbf{E}_{t}\left(\tilde{\mathrm{q}}_{t+1}+\hat{\mathrm{q}}_{t+1}^{\star}\right)+\Lambda_{12} \mathbf{E}_{t}\left(\hat{\tau}_{t}^{i}-\hat{\tau}_{t}^{j}\right)+\Lambda_{13} \mathbf{E}_{t}\left(\hat{\tau}_{t+1}^{i}-\hat{\tau}_{t+1}^{j}\right)=0
\end{aligned}
$$

for an appropriately defined set of composite parameters, $\Lambda$. Through this relation, movements in the efficient degrees of labor market tightness, in efficient levels of consumption, and in the efficient real exchange rate, force the policymaker to trade off efficiency gaps beyond what is called for by balancing inflation differences and the allocation of aggregate demand across the union. In this way, labor mobility complicates the setting of optimal monetary policy.

In total, the policymaker optimizes monetary policy by choosing 22 efficiency gaps to minimize (54) subject to 21 constraints. The full set of constraints as well as the first-order conditions are given in Appendix $\mathrm{H}$. 


\section{Calibration}

We calibrate the model in a three-step procedure. First, we set basic parameters to conventional values from the literature. Second, we choose a number of parameters affecting labor market outcomes to match a set of empirical targets for steady-state relations in the model, focusing on a zero-inflation non-stochastic steady state. And third, we find standard deviations to shocks in the model by matching a set of moments in the data. Parameter values are summarized in Table 1, while the standard deviations of all shocks to productivity, matching efficiency, and the marginal utility of consumption are reported in Table 2. We consider four deviations from optimal policy, assuming the monetary authority to follow the simple rule (48) with the specifications described in Table 3.

Following the business-cycle literature, we set the preference discount factor to $\beta=0.99$; the elasticity of substitution between consumption goods to $\epsilon=11$; price stickiness to $\delta=2 / 3$; and marginal returns to labor in production to $\alpha=1 / 3$. In our benchmark calibration, we set $\nu_{2}=1.5$, within the empirically relevant range of $[1.5,3.5]$ for interregional trade elasticities reported by Bilgic et al. (2002). The jobseparation rate $\rho=0.1$ is chosen so that jobs last on average for about 10 quarters (see e.g. Shimer, 2005). The bargaining power of firms is set to the conventional value of $\eta=0.4$ in line with estimates in Flinn (2006). Hence, from the Hosios (1990) condition, the elasticity of matches to vacancies is $\gamma=\eta=0.4$. This value for $\gamma$ corresponds to the midpoint of values typically used in the literature (see e.g. Gertler and Trigari, 2009). Parameter $\zeta \in(0,1)$ captures the fraction of leisure time forgone by the unemployed relative to the employed, as the unemployed search for jobs, develop skills, or collect unemployment benefits. We assume that this cost is the same for native and migrant searchers, because the extra cost of searching in the foreign labor market is captured by parameters $\alpha_{1}$ and $\nu_{1}$. Similar to Campolmi and Gnocchi (2016), $\zeta$ is calibrated to data from the American Time Use Survey (ATUS) for the period 2003-2006 as reported by Krueger and Mueller (2012). Table 4 shows time devoted to leisure, depending on the employment status, and the implied value for $\zeta$.

In the symmetric steady state, $\alpha_{1}$ defines the shares of migrant employment to total employment, migrant unemployment to total unemployment, and migrant searchers to total searchers, i.e. $\alpha_{1}=E^{i j} / E=U^{i j} / U=S^{i j} / S$. We therefore calibrate $\alpha_{1}$ to match the average share of gross migration flows to total employment over all U.S. states, as observed in the IRS data over the 1976-2016 period. Firms' search cost, $\kappa$, the utility parameter, $b$, and the matching efficiency, $\tau$, are set simultaneously to target (i) an employment rate of $\bar{E}=0.94$, computed using quarterly U.S. data from FRED II over the period 1976:Q1-2016:Q4, (ii) a job-filling rate of $\bar{q}=2 / 3$, and (iii) a vacancy cost per filled job as a fraction of the real wage $(\kappa / \bar{q}) /(W / P)=0.045$ (see Galí, 2010). For cases where unemployment benefits, $b^{U}$, are non-zero, we calibrate them to match a replacement rate of $b^{U} /(W / P)=0.4$.

Finally, the standard deviations of regional technology, matching efficiency, and preference shocks, as well as the elasticity of labor substitution $\left(\nu_{1}\right)$, are calibrated to 
minimize the average distance of simulated unconditional moments from their empirical counterparts with the serial correlation of all shocks fixed at 0.88 . The targeted data moments are the standard deviation of output, the relative standard deviation of employment, the correlation between output and employment, and (for the model with mobility) the relative volatility of gross migration flows. Under the model assumption that regions are symmetric, we match regional moments in the model to aggregate moments in quarterly U.S. data from the FRED II database for the period 1976:Q1 2016:Q4. Moments are calculated after applying a standard HP filter. Output volatility is expressed in terms of percentage standard deviations. The relative volatility of gross migration flows corresponds to the median over relative standard deviations of state-to-state labor flows as measured in IRS data.

Table 2 reports empirical and simulated moments for the models, both with and without labor mobility, assuming monetary policy following a simple instrument rule (R2, as defined in Table 3). In both cases, the model matches the targeted moments quite closely. Output volatility in the model and the data are near-identical, while the model somewhat overstates the relative volatility of employment, a common feature of many business cycle models. The correlation between output and employment is positive in the model as in the data, even if it is somewhat smaller in the model with mobility than in the model without it. Consequently, the model does well in capturing the negative correlations between output and unemployment and between employment and unemployment, a key feature of labor market dynamics. The reduced correlation between output and employment allows the model with mobility to better match the relative unemployment volatility. Finally, the model with mobility closely tracks relative gross migration volatility in the data as well as the correlations of migration flows with key aggregate variables. Overall, we consider the empirical fit of the model to be satisfactory for our welfare analysis.

\section{Macroeconomic dynamics with a mobile labor force}

This section studies macroeconomic dynamics following a set of economic disturbances. The objective is to shed light on how labor mobility affects macroeconomic adjustments in the monetary union. Specifically, we consider dynamics caused by shocks to region-specific productivity, matching efficiency, and household preferences, both in the full model with labor mobility and in the benchmark model with a geographically immobile labor force. Throughout, the model is calibrated according to Table 1. We draw two main conclusions. First, when monetary policy is conducted optimally, a mobile labor force clearly facilitates adjustments to all the region-specific shocks under consideration by closing welfare-relevant efficiency gaps. Second, the extent to which labor mobility helps absorb region-specific shocks when monetary policy is suboptimal depends on the type of shock and structural characteristics of regional economies such as nominal rigidities and openness to trade. 


\subsection{Technology shocks}

Figure 1 shows responses to an innovation to total factor productivity in region $i$. In the efficient allocation (shown in black lines with diamonds in both the left and the right panel), the social planner moves workers towards the more productive region $i$ to support an expansion of output. Responses in the market equilibrium are shaped by the monetary policy reaction function. If monetary policy follows a simple inflation-targeting rule (R2 as defined in Table 3), monetary policy is insufficiently accommodating to prevent a sharp deterioration in region $i$ 's labor market performance. ${ }^{14}$ When labor is mobile, households optimally allocate unmatched workers towards the relatively better-performing labor market in region $j$ in this case, as shown in the left panel Figure 1 (red lines with circles). ${ }^{15}$ Compared to the benchmark model without mobility (blue lines with stars), these mis-directed migration flows amplify both the inefficient contraction in employment in region $i$ and the inefficient expansions of employment and output in region $j$. In this sense, labor mobility becomes an additional source of cross-regional spillovers when prices are sticky. From a traditional stabilization perspective, focused on closing gaps in output and inflation, it may seem that labor mobility worsens a dilemma for monetary policy between accommodating region-specific changes in productivity and stabilizing activity in the rest of the union. But from a welfare perspective, mobility facilitates the adjustment process by allowing region $i$ to effectively export unemployment to region $j$, limiting an inefficient drop in labor market tightness and dampening disinflationary pressures. In region $j$, in turn, new job seekers help prevent overheating in the labor market and so reduce upward pressures on wages and ultimately prices. Despite the seemingly inefficient direction of labor flows, a mobile labor force helps absorb region-specific productivity shocks through this channel when monetary policy is suboptimal.

Optimal monetary policy is more aggressive in accommodating a region-specific productivity shock, as illustrated in the right panel of Figure 1, for both the benchmark and the full model. The optimizing policymaker therefore sustains employment in region $i$ at a level that allows wage prospects to increase with productivity. When labor is mobile, workers are induced to migrate from region $j$ towards the more productive region $i$ as in the efficient allocation. These flows further increase monetary policy's scope for accommodating the productivity shock in region $i$ by limiting the expansionary spillovers on region $j$. In this case, monetary policy and labor mobility pull together to improve macroeconomic outcomes.

\subsection{Matching efficiency shock}

Figure 2 illustrates how labor mobility and optimal monetary policy also work together in response to an innovation to matching efficiency in labor market $i$ (for both

\footnotetext{
${ }^{14}$ For a discussion of this effect in New Keynesian models, see e.g. Galí and Rabanal (2004), Balleer (2012), and Mandelman and Zanetti (2014).

${ }^{15}$ Such migration flows are consistent with the empirical evidence, e.g. in Saks and Wozniak (2011) and Hauser (2014).
} 
native and migrant workers). When matching technology improves temporarily, the desired number of workers can be hired with fewer vacancies. Without mobility (blue lines with stars), a fall in vacancy postings translates directly into looser labor market conditions in region $i$. As real wages fall, firms reduce prices. Monetary policy responds by reducing interest rates, stimulating activity across the union. As a result, the labor market tightens and the real wage increases in region $j$. When labor is mobile (red lines with circles), however, households respond to divergent labor market prospects by allocating more workers towards region $j$ until expected labor market conditions equalize across the two regions. Thus, net migration flows in the same direction as in the efficient allocation (black lines with diamonds), alleviating the monetary policy trade-offs between efficiency gaps in regional labor market.

\subsection{Preference shock}

The potential role of a mobile labor force in absorbing asymmetric effects of a regionspecific preference shock crucially depends on the degree of trade openness of regional economies. ${ }^{16}$ Figure 3 shows responses under optimal monetary policy to a temporary increase in the marginal utility of consumption in region $i$, driving up consumption in the region through intertemporal substitution. In our baseline calibration with $\alpha_{2}=0.5$, consumption bundles consist of domestically produced goods and imports in equal measure. The regional preference shock therefore causes aggregate demand, output, and employment to increase equally for all firms across the union, leaving no role for labor mobility to absorb the shock. Net migration is zero and impulse responses in the full model (shown in black lines with diamonds) coincide with dynamics in the benchmark model. In addition, with a uniform increase in demand, the shock does not call for an adjustment of relative prices. By a divine coincidence, the shock does not lead to a trade-off for monetary policy, either through (58) or (59). Consequently, monetary policy is able to perfectly replicate the efficient allocation.

If demand is biased towards domestically produced goods $\left(\alpha_{2}<0.5\right)$, by contrast, a regional preference shock reallocates aggregate demand towards goods produced in the region hit by the shock. As in the example emphasized by Mundell (1961), the shift in relative demand works to make the region's labor market tighter than in the rest of the monetary union. In this case, labor mobility may help to stabilize labor market conditions across the union and ease trade-offs for monetary policy in responding to both divergent labor market conditions and relative price adjustments. Figure 3 illustrates how responses in the full model (red circles) now differ from those in the benchmark model (blue stars) following a region-specific preference shock in region $i$. When labor is free to move across regional borders, households respond to divergent

\footnotetext{
${ }^{16}$ Farhi and Werning (2014-20105) make a similar point, showing that labor mobility helps the macroeconomic adjustment after region-specific demand shortfalls in a currency union if demand is external (i.e. regional production is specialized in tradeable goods), but not when it is internal (i.e. regional production relies on non-tradeable goods).
} 
labor market conditions and reallocate unmatched workers towards the relatively tight labor market in region $i$. The optimizing policymaker can now provide further support to an expansion in $i$ without causing overheating in region $j$ 's labor market.

\section{Evaluation of simple monetary policy rules}

In this section, we consider the welfare consequences of deviating from optimal policy by letting the monetary authority follow each of the versions of the simple rule (48) described in Table 3. The first simple rule (R1) is a strict inflation-targeting rule that requires monetary policy to fully stabilize union-wide producer price inflation at all times. The second rule (R2) is a simple version of inflation targeting. Monetary policy only leans against union-wide inflation when following this rule without stabilizing inflation fully. The third rule (R3) lets the policymaker lean against union-wide unemployment as well as inflation. Finally, the fourth rule (R4) is a representation of the kind of flexible inflation-targeting rules with interest rate smoothing that is often taken to represent actual monetary policy (e.g. Smets and Wouters, 2007). Welfare losses are measured by the unconditional expectation of period losses in (56) relative to optimal monetary policy and expressed in percent of steady-state consumption. To build intuition, we begin by considering the benchmark model without mobility, before turning to the full model with mobility.

\subsection{Monetary union without mobility}

Table 5 compares welfare losses along with standard deviations of selected regional macroeconomic variables for the different monetary policy rules in the benchmark economy with a fixed labor force. Optimal monetary policy, for which relative losses are zero by construction, is very successful in stabilizing both inflation and labor market variables. An inflation nutter somewhat overstabilizes inflation rates at the expense of higher volatility of welfare-relevant labor market variables. But the welfare costs are small and the strict inflation-targeting rule (R1) is close to optimal. ${ }^{17}$ The simple inflation targeting rule (R2), by contrast, generates sizable welfare losses. Monetary policy does not respond sufficiently forcefully to economic disturbances in this case, allowing both inflation and real variables to be quite volatile. Adding a moderate weight on unemployment (R3) significantly improves welfare by stabilizing efficiency gaps in labor market variables. Leaning against output fluctuation instead, as in the conventional instrument rule (R4), also improves welfare but by much less than leaning against labor market conditions directly. As in the closed economy analyzed by Ravenna and Walsh (2011), ignoring labor market frictions can lead to large welfare losses in the monetary union. Labor market fluctuations add to the volatility of inflation

\footnotetext{
${ }^{17}$ A unitary trade elasticity $\left(\nu_{2}=1\right)$ would make strict inflation stabilization (R2) optimal; see e.g. Pappa (2004). Notice also that full stabilization of the measure of union-wide inflation requires some variation in regional inflation rates.
} 
through firms' marginal costs in the Phillips curve. While marginal costs in models with a competitive labor market depend on the output gap alone, search frictions render marginal costs additionally dependent on labor market gaps. Stabilizing inflation in the presence of labor market frictions therefore requires closing both the output gap and gaps in the regional labor markets.

Overall, the near optimality of R1 suggests that the optimal degree of flexibility in inflation targeting is fairly modest despite the search and matching framework in the monetary union. At the same time, the good performance of R3 suggests that there are significant welfare gains from adding a weight on labor market developments when setting monetary policy in the absence of an ability to stabilize inflation fully.

\subsection{Monetary union with a mobile labor force}

Table 6 shows welfare losses and standard deviations for the full model with labor mobility. Strict inflation targeting $(\mathrm{R} 1)$ is slightly more costly relative to optimal policy than in the benchmark model. As we have discussed above, free movement of workers introduces additional trade-offs for the common monetary authority because households do not fully internalize the effects of their migration decisions on aggregate labor market outcomes. Ignoring these trade-offs by targeting inflation strictly incurs extra welfare costs. As the table shows, however, these additional costs are low. For the three other suboptimal rules, labor mobility is seen to reduce welfare losses relative to optimal policy significantly compared to the benchmark model. In a nutshell, a mobile labor force alleviates some of the side effects of suboptimal monetary policy by closing inefficient gaps in regional labor markets through migration. But the case for leaning against labor market imbalances is stronger. When labor is mobile, a flexible inflation-targeting rule with some weight on unemployment (R3) is close to optimal and outperforms strict inflation targeting.

Figure 4 further illustrates how a weight on unemployment may reduce welfare losses under a simple inflation targeting rule. The figure shows the sensitivity of relative welfare losses under R3 to the weight on inflation, $\Gamma_{\pi}$, and the weight on unemployment, $\Gamma_{u}$. Both in the benchmark model (left panel) and in the full model (right panel), increasing $\Gamma_{\pi}$ reduces welfare losses for any given $\Gamma_{u}$, and relative welfare losses become increasingly insensitive to small changes in the weight on unemployment. By contrast, for relatively low weights on inflation, leaning slightly against unemployment significantly reduces welfare losses in both models. As inflation is very costly, however, increasing the weight on unemployment beyond moderate levels can quickly drive welfare losses back up. For $\Gamma_{\pi}=1.5$, for example, the optimal weight on unemployment is roughly $\Gamma_{u}=-0.1$ in both models.

In sum, the optimal degree of flexibility in inflation targeting remains modest despite inefficiencies in households' migration behaviors. But migration flows strengthen the case for leaning against labor market imbalances in the monetary union. A simple inflation-targeting rule with a moderate weight on unemployment comes close to minimizing welfare losses in the monetary union. 


\section{Conclusion}

Labor mobility complicates the setting of monetary policy in a monetary union. An optimizing policymaker shows somewhat greater flexibility in inflation targeting by putting weight on inefficient migration flows in addition to regional labor market

conditions. Nevertheless, labor mobility facilitates macroeconomic adjustments in a monetary union by closing inefficiency gaps in regional labor markets. The exact role played by labor mobility in macroeconomic adjustments depends crucially on the underlying macroeconomic disturbances, the structure of regional economies, and the monetary policy regime. But overall, the welfare costs of pursuing suboptimal monetary policy strategies are smaller when labor is free to cross regional borders. A simple instrument rule with a moderate weight on unemployment stabilization is close to optimal.

While labor mobility reduces welfare losses under the monetary policy regimes we consider, we leave a thorough assessment of the effects-free movement on the case for joining a monetary union and thus giving up an independent monetary policy for future research. Another natural extension of this paper would be to relax the assumption that the currency union consists of two symmetric regions. We consider assessing the role of a mobile labor force across regions differing in terms of their exposure to shocks - e.g. due to an unequal industry composition-or differing in terms of their labor market structures as important avenues for future research. 


\section{References}

Balleer, Almut, "New evidence, old puzzles: Technology shocks and labor market dynamics," Quantitative Economics, 2012, 3 (3), 363-392.

Bandeira, Guilherme, Jordi Caballé, and Eugenia Vella, "Should I stay or should I go? Austerity, unemployment and migration," ADEMU Working Paper Series, 2018, 83.

Benigno, Pierpaolo, "Optimal monetary policy in a currency area," Journal of International Economics, 2004, 63, 293-320.

Bilgic, Abdulbaki, Stephen King, Aaron Lusby, and Dean Schreiner, "Estimates of US regional commodity trade elasticities of substitution," Journal of Regional Analysis and Policy, 2002, 32 (2), 31-50.

Blanchard, Olivier and Jordi Galí, "Labor markets and monetary policy: A New Keynesian model with unemployment," American Economic Journal: Macroeconomics, 2010, 2 (2), 1-30.

Calvo, Guillermo, "Staggered prices in a utility maximizing framework," Journal of Monetary Economics, 1983, 12 (3), 383-398.

Campolmi, Alessia and Stefano Gnocchi, "Labor market participation, unemployment and monetary policy," Journal of Monetary Economics, 2016, 79, 17-29.

Dellas, Harris and George Tavlas, "An optimum-currency-area odyssey," Journal of International Money and Finance, 2009, 28 (7), 1117-1137.

Farhi, Emmanuel and Iván Werning, "Labor mobility within currency unions," NBER Working Paper Series, 2014-20105.

Flinn, Christopher, "Minimum wage effects on labor market outcomes under search, matching, and endogenous contact rates," Econometrica, 2006, 74 (4), 1013-1062.

Galí, Jordi, "Monetary policy and unemployment," Handbook of Monetary Economics, 2010, 3, 487-546.

_, Monetary Policy, Inflation, and the Business Cycle: An Introduction to the New Keynesian Framework and Its Applications, Princeton University Press, 2015.

- and Pau Rabanal, "Technology shocks and aggregate fluctuations: How well does the real business cycle model fit postwar US data?," NBER Macroeconomics Annual, 2004, 19, 225-288.

Gertler, Mark and Antonella Trigari, "Unemployment fluctuations with staggered Nash wage bargaining," Journal of Political Economy, 2009, 117 (1), 38-86. 
Greenwald, Bruce and Joseph Stiglitz, "Externalities in economies with imperfect information and incomplete markets," The Quarterly Journal of Economics, 1986, 101 (2), 229-264.

Hauser, Daniela, "Technology shocks, labour mobility and aggregate fluctuations," Bank of Canada Working Paper, 2014, 4.

Hosios, Arthur, "On the efficiency of matching and related models of search and unemployment," The Review of Economic Studies, 1990, 57 (2), 279-298.

House, Christopher, Christian Proebsting, and Linda Tesar, "Quantifying the benefit of labor mobility in a currency union," NBER Working Paper Series, 2018, 25347.

Krueger, Alan and Andreas Mueller, "The lot of the unemployed: A time use perspective," Journal of the European Economic Association, 2012, 10 (4), 765-794.

Lewis, John and Matt Swannel, "The macroeconomic determinants of migration," Bank of England Staff Working Paper, 2018-729.

Lkhagvasuren, Damba, "Big locational unemployment differences despite high labor mobility," Journal of Monetary Economics, 2012, 59 (8), 798-814.

Mandelman, Federico and Francesco Zanetti, "Flexible prices, labor market frictions and the response of employment to technology shocks," Labour Economics, 2014, 26, 94-102.

Mundell, Robert, "A theory of optimum currency areas," American Economic Review, 1961, 53, 657-665.

Pappa, Evi, "Do the ECB and the fed really need to cooperate? Optimal monetary policy in a two-country world," Journal of Monetary Economics, 2004, 51 (4), 753779 .

Ravenna, Federico and Carl Walsh, "Welfare-based optimal monetary policy with unemployment and sticky prices: A linear-quadratic framework," American Economic Journal: Macroeconomics, 2011, 3 (2), 130-162.

Rotemberg, Julio and Michael Woodford, "Interest rate rules in an estimated sticky price model," in John B. Taylor, ed., Monetary Policy Rules, National Bureau of Economic Research, 1999, pp. 57-126.

Saks, Raven and Abigail Wozniak, "Labor reallocation over the business cycle: New evidence from internal migration," Journal of Labor Economics, 2011, 29 (4), 697-739. 
Shimer, Robert, "The cyclical behavior of equilibrium unemployment and vacancies," American Economic Review, 2005, 95 (1), 25-49.

Smets, Frank and Rafael Wouters, "Shocks and frictions in US business cycles: A Bayesian DSGE approach," American Economic Review, 2007, 97 (3), 586-606.

Smith, Christie and Christoph Thoenissen, "Migration and business cycle dynamics," Reserve Bank of New Zealand Discussion Paper Series, 2018, DP2018/07.

Thomas, Carlos, "Search and matching frictions and optimal monetary policy," Journal of Monetary Economics, 2008, 55, 936-956.

Walsh, Carl, "Multiple objectives and central bank tradeoffs under flexible inflation targeting," CESifo Working Paper No. 5097, 2014.

Woodford, Michael, "Commentary: How should monetary policy be conducted in an era of price stability?," New Challenges for Monetary Policy, Federal Reserve Bank of Kansas City, 1999, pp. 277-316.

_, Interest and Prices: Foundations of a Theory of Monetary Policy, Princeton University Press, 2011. 


\section{Tables and Figures}

\begin{tabular}{l|ccc} 
Parameter & Mnemonic & Value & Target/Source \\
\hline \hline & $\beta$ & 0.99 & $4 \%$ avg. real return \\
Discount factor & $\epsilon$ & 11 & $10 \%$ price markup \\
Elasticity of substitution & $\delta$ & $2 / 3$ & 8 months exp. duration \\
Calvo price stickiness & $\alpha$ & $1 / 3$ & Galí $(2015)$ \\
Returns to scale in production & $\nu_{2}$ & 1.50 & Bilgic et al. $(2002)$ \\
Trade elasticity & $\rho$ & 0.10 & Shimer $(2005)$ \\
Job-separation rate & $\eta$ & 0.40 & Flinn $(2006)$ \\
Firms' bargaining power & $\gamma$ & 0.40 & Hosios condition \\
Elasticity of matches to vacancies & $\zeta$ & 0.735 & ATUS data \\
Unemployment disutility & & & \\
& & & \\
\hline Home bias in labor & $\alpha_{1}$ & 0.0779 & IRS data \\
Elasticity of labor substitution & $\nu_{1}$ & -9.5 & IRS data \\
Vacancy cost & $\kappa$ & 0.03 & 0.94 employment rate \\
Unemployment benefit & $b^{U}$ & 0.27 & $\frac{b^{U}}{W / P}=0.4$ \\
Matching efficiency & $\tau$ & 0.64 & $2 / 3$ job-filling rate \\
Utility parameter & $b$ & 0.97 & $\frac{\kappa / q}{W / P}=0.045$ \\
& & & \\
\hline
\end{tabular}

Table 1: Benchmark calibration 


\begin{tabular}{l|ccc} 
Unconditional Moments & Data & Mobility & No Mobility \\
\hline \hline Output volatility & 1.37 & 1.36 & 1.37 \\
Relative employment volatility & 0.75 & 0.95 & 0.97 \\
Correlation output and employment & 0.84 & 0.23 & 0.59 \\
Relative volatility gross migration & 4.23 & 4.48 & n.a. \\
\hline Correlation output and unemployment & -0.87 & -0.37 & -0.59 \\
Correlation employment and unemployment & -0.94 & -0.74 & -1.00 \\
Correlation output and wages & 0.91 & 0.43 & 0.55 \\
Relative unemployment rate volatility & 7.94 & 14.00 & 17.10 \\
Correlation gross migration and output & 0.22 & 0.18 & n.a. \\
Correlation gross migration and wages & 0.14 & 0.09 & n.a. \\
\hline & & & \\
Calibrated Parameters & & & \\
\hline \hline$\sigma_{a}$ & & 0.0105 & 0.0080 \\
$\sigma_{m e}$ & & 0.0432 & 0.0998 \\
$\sigma_{z}$ & -9.5 & n.a. \\
$\nu_{1}$ & &
\end{tabular}

Table 2: Selected unconditional moments and calibrated parameters in the benchmark model without mobility and in the full model with a geographically mobile labor force 


\begin{tabular}{l|l|cccc} 
Rule & Description & $\rho_{r}$ & $\Gamma_{\pi}$ & $\Gamma_{u}$ & $\Gamma_{y}$ \\
\hline \hline R1 & Strict inflation targeting & 0 & $\infty$ & 0 & 0 \\
R2 & Simple inflation targeting & 0 & 1.5 & 0 & 0 \\
R3 & Weight on unemployment & 0 & 1.5 & -0.125 & 0 \\
R4 & Flexible inflation targeting & 0.8 & 2 & 0 & 0.125
\end{tabular}

Table 3: Different monetary policy regimes under consideration, given the generic rule $\hat{r}_{t}=\rho_{r} \hat{r}_{t-1}+\left(1-\rho_{r}\right)\left(\Gamma_{\pi} \hat{\pi}_{t}+\Gamma_{u} \hat{u}_{t}+\Gamma_{y} \hat{y}_{t}\right)$ 


\begin{tabular}{|l|c|c|}
\hline Leisure Time & $\begin{array}{c}\text { 2003-2006 } \\
\text { Definition 1 }\end{array}$ & $\begin{array}{c}\text { 2003-2006 } \\
\text { Definition 2 }\end{array}$ \\
\hline Maximum & 827.5 & 827.5 \\
\hline Unemployed & 450 & 344 \\
\hline Employed & 293 & 186 \\
\hline \hline Search cost $\zeta$ & 0.71 & 0.76 \\
\hline
\end{tabular}

Table 4: Calibration of search cost: Data on time allocated to leisure time (in minutes per average weekday) are from the ATUS and were collected over the period 2003-2006. The maximum leisure time is computed as total available time minus average time spent on sleeping, personal care, and eating, averaged over employed and unemployed persons. Definition 1 for leisure time accounts for the following activities: Leisure and socializing; voluntary, religious and civic activities; sport; travel. Definition 2 encompasses time spent on leisure and socializing only. $\zeta$ is defined as the difference between the maximum leisure time and the leisure time of unemployed relative to the difference between the maximum leisure time and the leisure time of employed, e.g. for definition 1, $\zeta=(827.5-450) /(827.5-293)=0.71$. For our benchmark calibration, we set $\zeta$ to the average value over the two definitions of maximum leisure time, i.e. $\zeta=0.735$. 


\begin{tabular}{|l|c|c|c|c|c|}
\cline { 2 - 6 } \multicolumn{1}{c|}{} & Optimal & R1 & R2 & R3 & R4 \\
\hline Losses & 0 & 0.04 & 1.91 & 0.08 & 1.04 \\
\hline$\sigma_{\pi_{H}}$ & 0.003 & 0.002 & 0.016 & 0.003 & 0.010 \\
\hline$\sigma_{e}$ & 0.001 & 0.008 & 0.011 & 0.008 & 0.018 \\
\hline$\sigma_{\theta}$ & 0.021 & 0.143 & 0.208 & 0.146 & 0.389 \\
\hline$\sigma_{c}$ & 0.000 & 0.003 & 0.006 & 0.003 & 0.010 \\
\hline$\sigma_{c_{H}}$ & 0.000 & 0.005 & 0.007 & 0.005 & 0.011 \\
\hline$\sigma_{c_{F}}$ & 0.000 & 0.005 & 0.007 & 0.005 & 0.011 \\
\hline$\sigma_{l}$ & 0.013 & 0.122 & 0.182 & 0.125 & 0.282 \\
\hline
\end{tabular}

Table 5: Benchmark No Mobility Model: Union-wide welfare losses and unconditional moments for different policy rules (see Table 3). Welfare losses are expressed relative to the optimal policy, in percent of steady-state consumption.

\begin{tabular}{|l|c|c|c|c|c|}
\cline { 2 - 6 } \multicolumn{1}{c|}{} & Optimal & R1 & R2 & R3 & R4 \\
\hline Losses & 0 & 0.05 & 0.91 & 0.01 & 0.45 \\
\hline$\sigma_{\pi_{H}}$ & 0.001 & 0.001 & 0.012 & 0.003 & 0.008 \\
\hline$\sigma_{e}$ & 0.001 & 0.009 & 0.010 & 0.009 & 0.013 \\
\hline$\sigma_{\theta}$ & 0.027 & 0.075 & 0.098 & 0.076 & 0.240 \\
\hline$\sigma_{c}$ & 0.000 & 0.000 & 0.002 & 0.000 & 0.006 \\
\hline$\sigma_{c_{H}}$ & 0.008 & 0.006 & 0.006 & 0.006 & 0.008 \\
\hline$\sigma_{c_{F}}$ & 0.008 & 0.006 & 0.006 & 0.006 & 0.008 \\
\hline$\sigma_{l}$ & 0.015 & 0.012 & 0.057 & 0.016 & 0.154 \\
\hline
\end{tabular}

Table 6: Full Model with Mobility: Union-wide welfare losses and unconditional moments for different policy rules (see Table 3). Welfare losses are expressed relative to the optimal policy, in percent of steady-state consumption. 

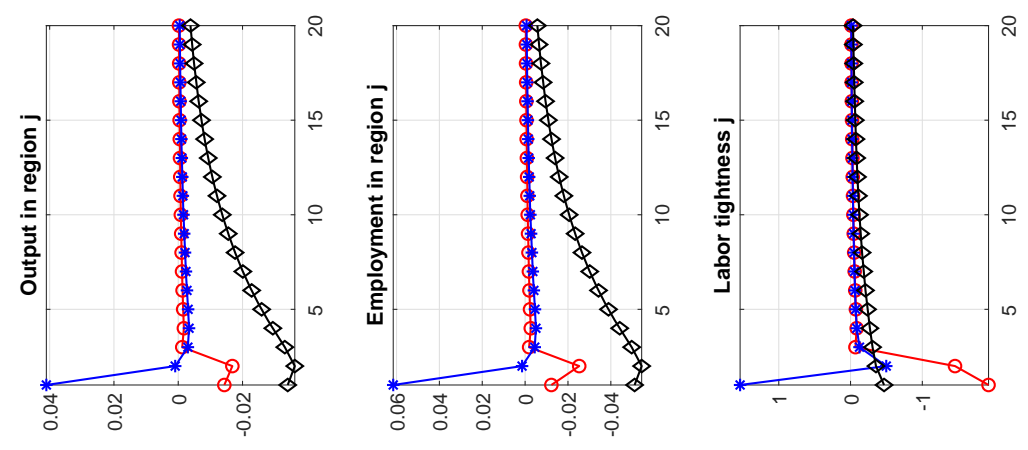

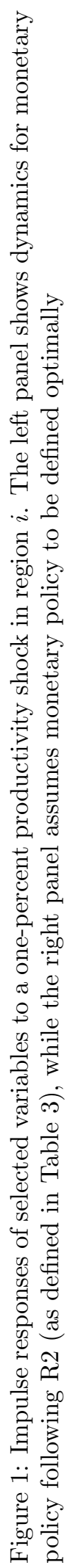
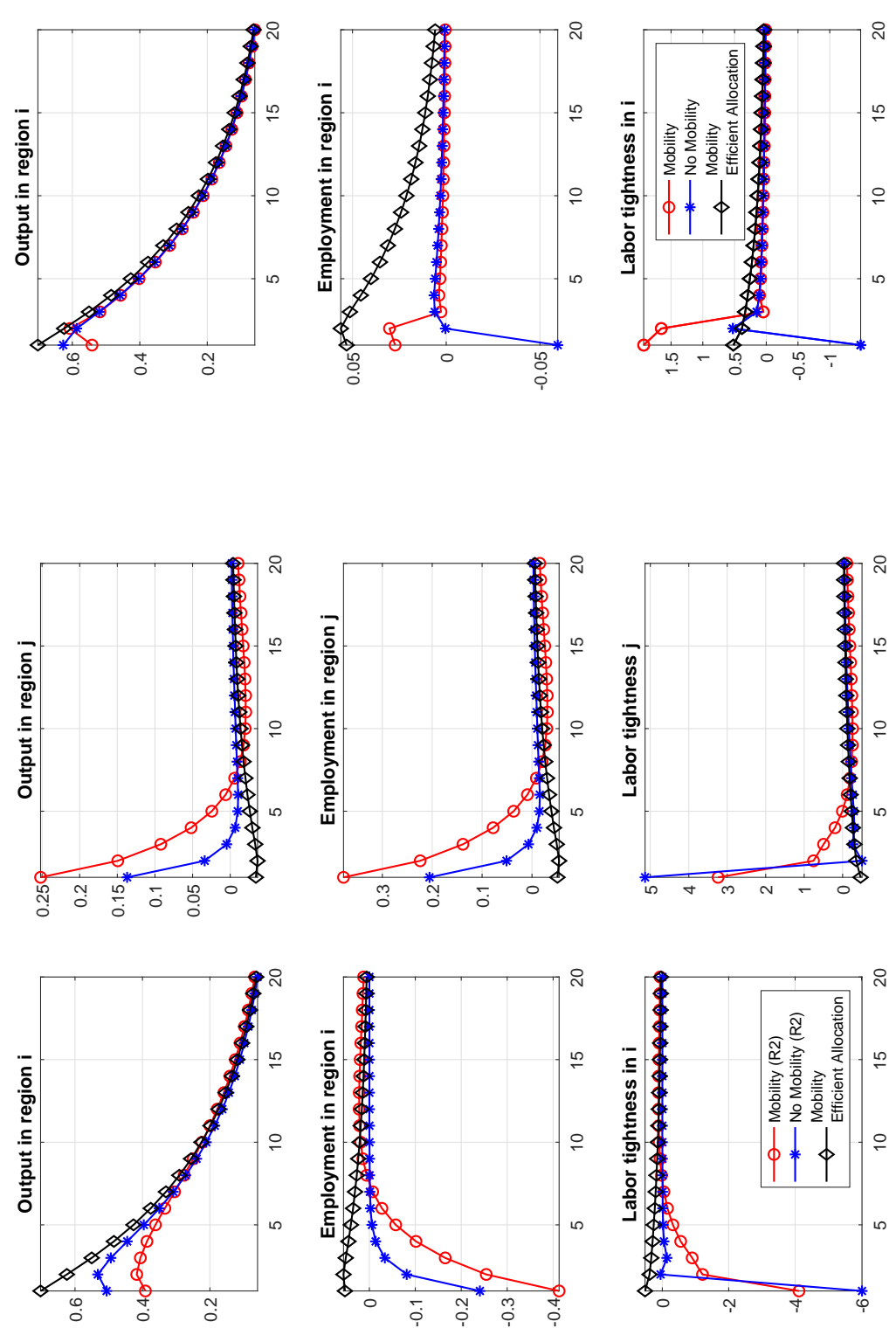

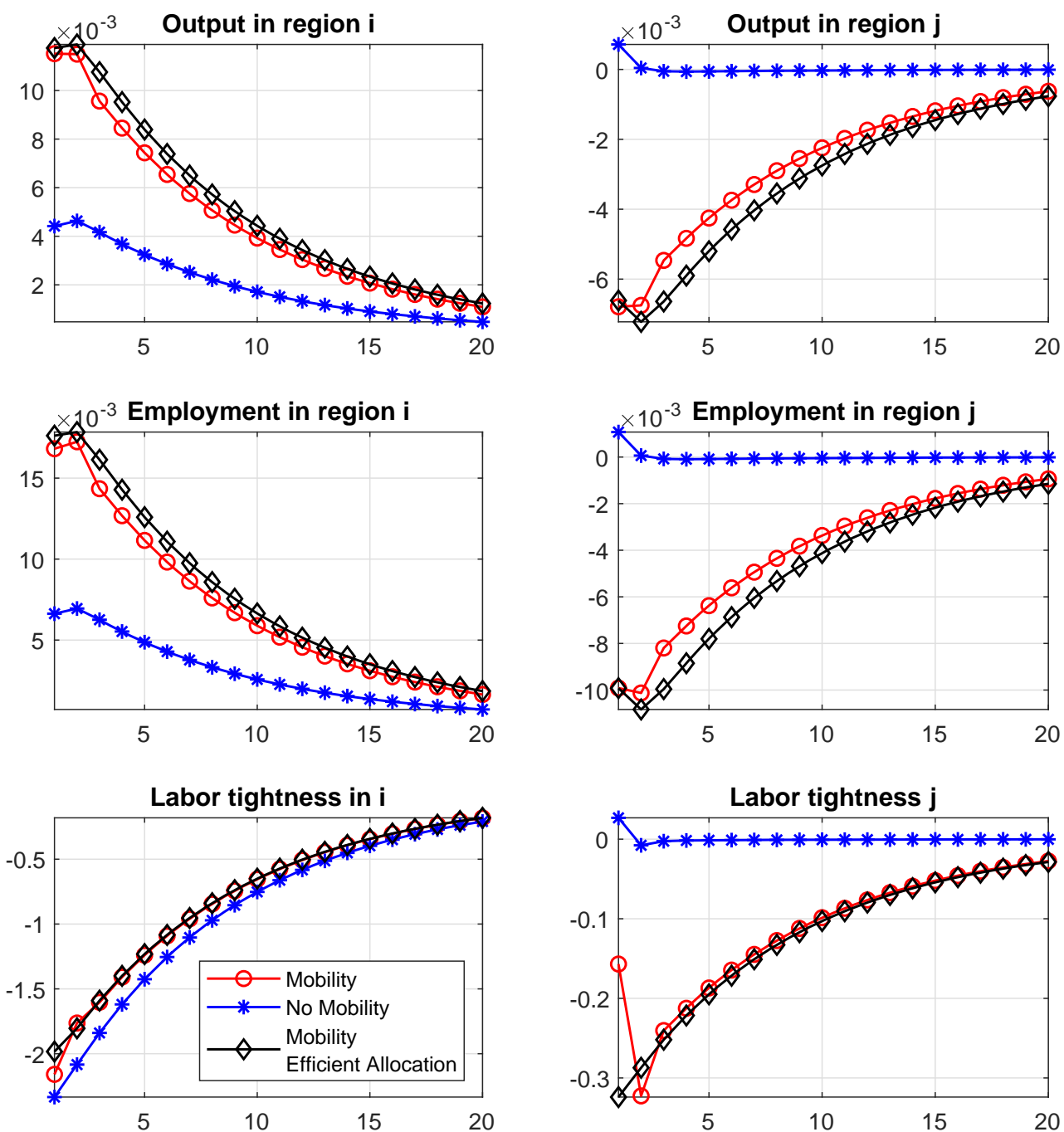

Figure 2: Impulse responses of selected variables to a one-percent positive matching-efficiency shock in region $i$ when monetary policy is conducted optimally 

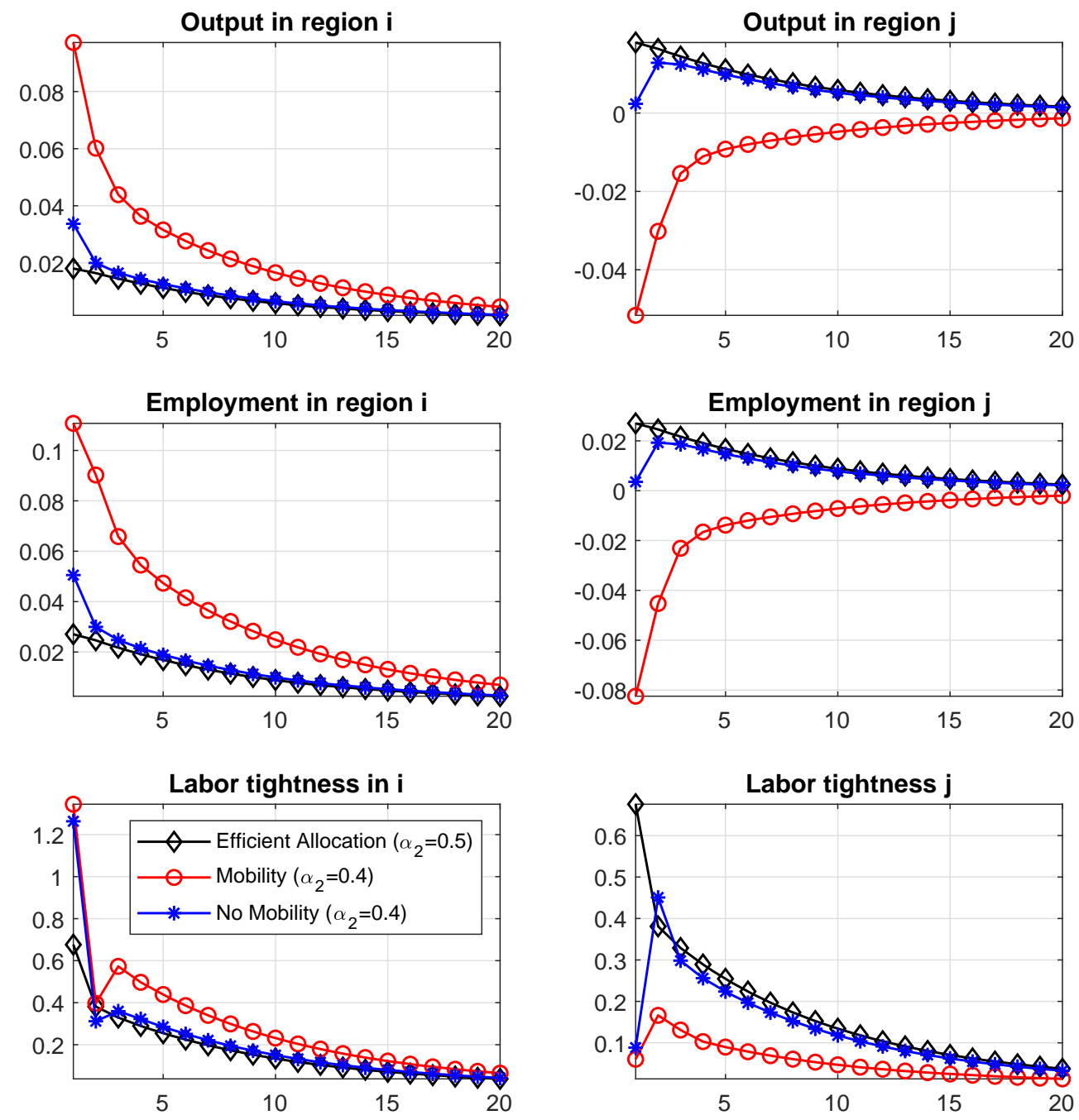

Figure 3: Impulse responses of selected variables to a one-percent positive preference shock in region $i$ when monetary policy is conducted optimally 

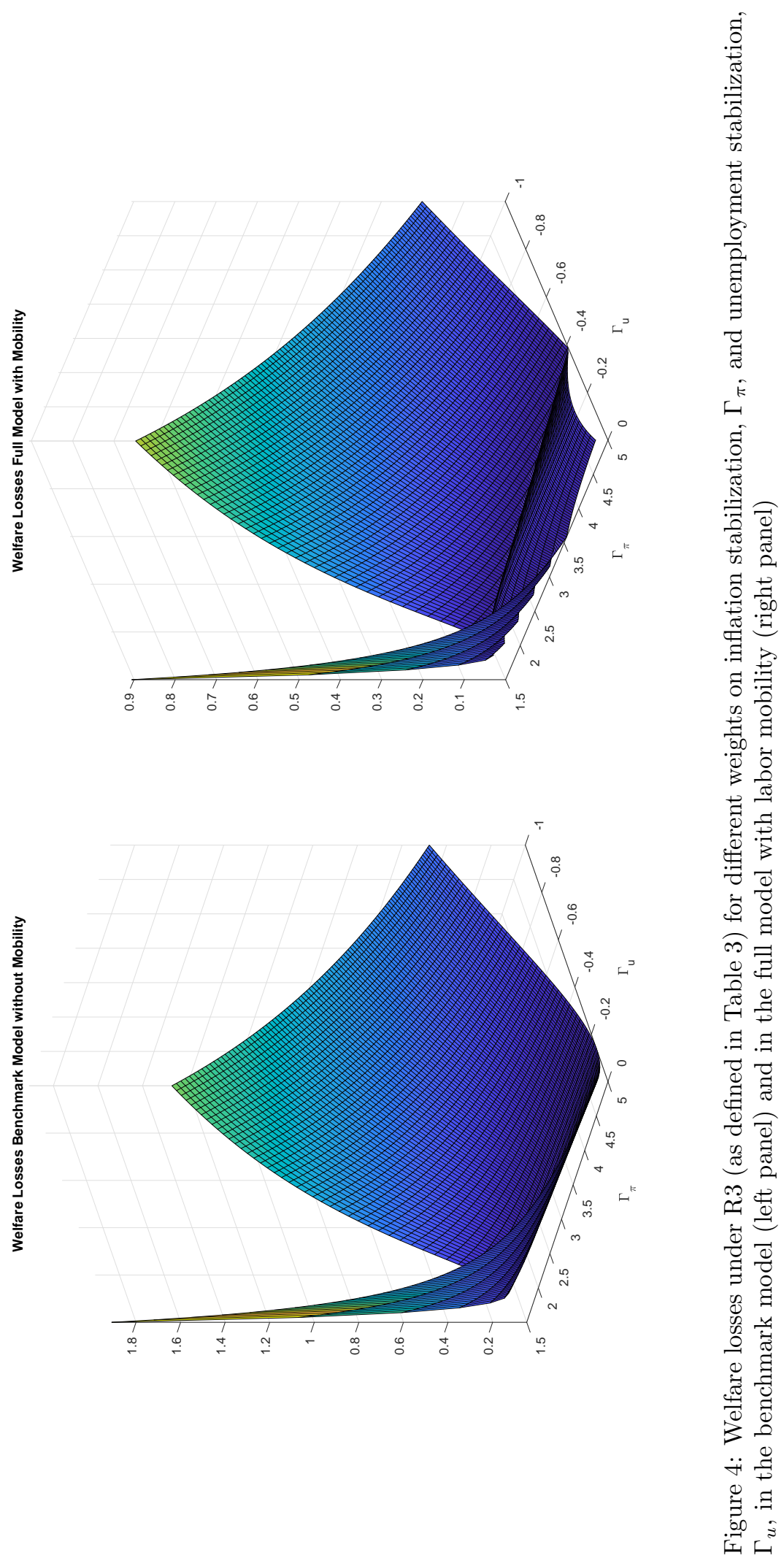


\section{Appendix A. Households' value function}

The Bellman equation associated to the optimization problem of the representative household in region $i$ states as follows:

$$
\mathcal{V}_{t}^{i}\left(E_{t-1}^{i i}, E_{t-1}^{i j}\right)=\max _{\Omega_{t}^{i}, E_{t}^{i i}, E_{t}^{i j}}\left\{\mathcal{U}_{t}^{i}\left[C_{t}^{i}(.), l_{t}^{i}(.)\right]+\beta \mathbf{E}_{\mathbf{t}}\left\{\mathcal{V}_{t+1}^{i}\right\}\right\}
$$

The constraints the household takes into account are

$$
\begin{aligned}
l_{t}^{i} & =1-\left[\left(1-\alpha_{1}\right)^{1 / \nu_{1}}\left(E_{t}^{i i}+\zeta U_{t}^{i i}\right)^{\frac{\nu_{1}-1}{\nu_{1}}}+\left(\alpha_{1}\right)^{1 / \nu_{1}}\left(E_{t}^{i j}+\zeta U_{t}^{i j}\right)^{\frac{\nu_{1}-1}{\nu_{1}}}\right]^{\frac{\nu_{1}}{\nu_{1}-1}} \\
E_{t}^{i i} & =(1-\rho)\left(1-f_{t}^{i i} \Omega_{t}^{i}\right) E_{t-1}^{i i}+f_{t}^{i i} \Omega_{t}^{i}\left(1-(1-\rho) E_{t-1}^{i j}\right) \\
E_{t}^{i j} & =(1-\rho)\left(1-f_{t}^{i j}\left(1-\Omega_{t}^{i}\right)\right) E_{t-1}^{i j}+f_{t}^{i j}\left(1-\Omega_{t}^{i}\right)\left(1-(1-\rho) E_{t-1}^{i i}\right) \\
U_{t}^{i i} & =\left(1-f_{t}^{i i}\right) \Omega_{t}^{i}\left[1-(1-\rho) E_{t-1}^{i i}-(1-\rho) E_{t-1}^{i j}\right] \\
U_{t}^{i j} & =\left(1-f_{t}^{i j}\right)\left(1-\Omega_{t}^{i}\right)\left[1-(1-\rho) E_{t-1}^{i i}-(1-\rho) E_{t-1}^{i j}\right] \\
C_{t}^{i} & =\frac{D_{t-1}^{i}}{P_{t-1}^{i}} \frac{P_{t-1}^{i}}{P_{t}^{i}}-R_{t}^{-1} \frac{D_{t}^{i}}{P_{t}^{i}}+\frac{W_{t}^{i i}}{P_{t}^{i}} E_{t}^{i i}+b^{U} U_{t}^{i i}+\frac{W_{t}^{i j}}{P_{t}^{i}} E_{t}^{i j}+\frac{P_{t}^{j}}{P_{t}^{i}} b^{U} U_{t}^{i j}+\frac{T_{t}^{i}}{P_{t}^{i}} \\
1 & =E_{t}^{i i}+U_{t}^{i i}+E_{t}^{i j}+U_{t}^{i j}
\end{aligned}
$$

Notice that equations (A.3), (A.4), (A.5), (A.6), and (A.8) make a system of 5 equations and 5 variables $\left(E_{t}^{i i}, E_{t}^{i j}, U_{t}^{i i}, U_{t}^{i j}\right.$, and $\left.\Omega_{t}^{i}\right)$, where the fifth equation is implied by the first four. For example, summing up (A.3), (A.4), (A.5), and (A.6) is equal to 1 and thus yields (A.8). In what follows, equation (A.8) is thus not explicitly taken into account in households' maximization problem.

Appendix A.1. Optimal migration decision

Households' first-order condition with respect to $\Omega_{t}^{i}$, i.e. the optimal migration decision, implies

$$
\begin{aligned}
\frac{\partial \mathcal{V}_{t}^{i}}{\partial E_{t}^{i i}} S_{H, t}^{i} f_{t}^{i i}+\frac{\partial \mathcal{V}_{t}^{i}}{\partial U_{t}^{i i}} S_{H, t}^{i}\left(1-f_{t}^{i i}\right) & =\frac{\partial \mathcal{V}_{t}^{i}}{\partial E_{t}^{i j}} S_{H, t}^{i} f_{t}^{i j}+\frac{\partial \mathcal{V}_{t}^{i}}{\partial U_{t}^{i j}} S_{H, t}^{i}\left(1-f_{t}^{i j}\right) \\
\frac{\partial \mathcal{V}_{t}^{i}}{\partial E_{t}^{i i}} f_{t}^{i i}+\frac{\partial \mathcal{V}_{t}^{i}}{\partial U_{t}^{i i}}\left(1-f_{t}^{i i}\right) & =\frac{\partial \mathcal{V}_{t}^{i}}{\partial E_{t}^{i j}} f_{t}^{i j}+\frac{\partial \mathcal{V}_{t}^{i}}{\partial U_{t}^{i j}}\left(1-f_{t}^{i j}\right) \\
\frac{\partial \mathcal{U}_{t}^{i}}{\partial E_{t}^{i i}} f_{t}^{i i}+\frac{\partial \mathcal{U}_{t}^{i}}{\partial U_{t}^{i i}}\left(1-f_{t}^{i i}\right) & =\frac{\partial \mathcal{U}_{t}^{i}}{\partial E_{t}^{i j}} f_{t}^{i j}+\frac{\partial \mathcal{U}_{t}^{i}}{\partial U_{t}^{i j}}\left(1-f_{t}^{i j}\right),
\end{aligned}
$$

which can be rewritten as

$$
\begin{gathered}
U_{c, t}^{i}\left\{\left[\frac{\partial C_{t}^{i}}{\partial E_{t}^{i i}}+\frac{U_{l, t}^{i}}{U_{c, t}^{i}} \frac{\partial l_{t}^{i}}{\partial E_{t}^{i i}}\right] f_{t}^{i i}+\left[\frac{\partial C_{t}^{i}}{\partial U_{t}^{i i}}+\frac{U_{l, t}^{i}}{U_{c, t}^{i}} \frac{\partial l_{t}^{i}}{\partial U_{t}^{i i}}\right]\left(1-f_{t}^{i i}\right)\right\}= \\
U_{c, t}^{i}\left\{\left[\frac{\partial C_{t}^{i}}{\partial E_{t}^{i j}}+\frac{U_{l, t}^{i}}{U_{c, t}^{i}} \frac{\partial l_{t}^{i}}{\partial E_{t}^{i j}}\right] f_{t}^{i j}+\left[\frac{\partial C_{t}^{i}}{\partial U_{t}^{i j}}+\frac{U_{l, t}^{i}}{U_{c, t}^{i}} \frac{\partial l_{t}^{i}}{\partial U_{t}^{i j}}\right]\left(1-f_{t}^{i j}\right)\right\}
\end{gathered}
$$


and further states as follows:

$$
\begin{aligned}
& f_{t}^{i i} {\left[\frac{W_{t}^{i i}}{P_{t}^{i}}-\frac{U_{l, t}^{i}}{U_{c, t}^{i}}\left(1-\alpha_{1}\right)^{1 / \nu_{1}}\left(\frac{E_{t}^{i i}+\zeta U_{t}^{i i}}{1-l_{t}^{i}}\right)^{-1 / \nu_{1}}\right]+} \\
&\left(1-f_{t}^{i i}\right)\left[b^{U}-\zeta \frac{U_{l, t}^{i}}{U_{c, t}^{i}}\left(1-\alpha_{1}\right)^{1 / \nu_{1}}\left(\frac{E_{t}^{i i}+\zeta U_{t}^{i i}}{1-l_{t}^{i}}\right)^{-1 / \nu_{1}}\right]= \\
& f_{t}^{i j}\left[\frac{W_{t}^{i j}}{P_{t}^{j}} Q_{t}^{i}-\frac{U_{l, t}^{i}}{U_{c, t}^{i}}\left(\alpha_{1}\right)^{1 / \nu_{1}}\left(\frac{E_{t}^{i j}+\zeta U_{t}^{i j}}{1-l_{t}^{i}}\right)^{-1 / \nu_{1}}\right]+ \\
&\left(1-f_{t}^{i j}\right)\left[Q_{t}^{i} b^{U}-\zeta \frac{U_{l, t}^{i}}{U_{c, t}^{i}}\left(\alpha_{1}\right)^{1 / \nu_{1}}\left(\frac{E_{t}^{i j}+\zeta U_{t}^{i j}}{1-l_{t}^{i}}\right)^{-1 / \nu_{1}}\right] .
\end{aligned}
$$

The same optimality condition results from a setup where households are choosing the searchers to be sent to either of the two labor markets $\left(S_{t}^{i i}\right.$ and $\left.S_{t}^{i j}\right)$ directly. Specifically, given the pool of searching members, $S_{H, t}^{i}$, instead of choosing $\Omega_{t}^{i}$, households would optimally decide on $S_{t}^{i i}$, which in turn pins down $S_{t}^{i j}$.

Appendix A.2. Value of native workers

The first-order condition with respect to $E_{t}^{i i}$ states as

$$
\frac{\partial \mathcal{U}_{t}^{i}}{\partial E_{t}^{i i}}=U_{c, t}^{i} \frac{\partial C_{t}^{i}}{\partial E_{t}^{i i}}+U_{l, t}^{i} \frac{\partial l_{t}^{i}}{\partial E_{t}^{i i}}=U_{c, t}^{i}[\frac{W_{t}^{i i}}{P_{t}^{i}}-\underbrace{\frac{U_{l, t}^{i}}{U_{c, t}^{i}}\left(1-\alpha_{1}\right)^{1 / \nu_{1}}\left(\frac{E_{t}^{i i}+\zeta U_{t}^{i i}}{1-l_{t}^{i}}\right)^{-1 / \nu_{1}}}_{\text {disutility of working as } E_{t}^{i i}}] .
$$

The current-period value of being native employed (in terms of consumption) is defined as the difference between the value of being matched to a firm and gaining wage $W_{t}^{i i} / P_{t}^{i}$ and the disutility attached to working as a native in labor market $i$. The first-order condition with respect to $E_{t-1}^{i i}$ :

$$
\begin{aligned}
\frac{\partial \mathcal{V}_{t}^{i}}{\partial E_{t-1}^{i i}}= & \frac{\partial \mathcal{V}_{t}^{i}}{\partial E_{t}^{i i}} \frac{\partial E_{t}^{i i}}{\partial E_{t-1}^{i i}}+\frac{\partial \mathcal{V}_{t}^{i}}{\partial E_{t}^{i j}} \frac{\partial E_{t}^{i j}}{\partial E_{t-1}^{i i}}+\frac{\partial \mathcal{V}_{t}^{i}}{\partial U_{t}^{i i}} \frac{\partial U_{t}^{i i}}{\partial E_{t-1}^{i i}}+\frac{\partial \mathcal{V}_{t}^{i}}{\partial U_{t}^{i j}} \frac{\partial U_{t}^{i j}}{\partial E_{t-1}^{i i}}= \\
& \frac{\partial \mathcal{U}_{t}^{i}}{\partial E_{t}^{i i}} \frac{\partial E_{t}^{i i}}{\partial E_{t-1}^{i i}}+\frac{\partial \mathcal{U}_{t}^{i}}{\partial E_{t}^{i j}} \frac{\partial E_{t}^{i j}}{\partial E_{t-1}^{i i}}+\frac{\partial \mathcal{U}_{t}^{i}}{\partial U_{t}^{i i}} \frac{\partial U_{t}^{i i}}{\partial E_{t-1}^{i i}}+\frac{\partial \mathcal{U}_{t}^{i}}{\partial U_{t}^{i j}} \frac{\partial U_{t}^{i j}}{\partial E_{t-1}^{i i}}= \\
U_{c, t}^{i} & {\left[\frac{\partial C_{t}^{i}}{\partial E_{t}^{i i}}+\frac{U_{l, t}^{i}}{U_{c, t}^{i}} \frac{\partial l_{t}^{i}}{\partial E_{t}^{i i}}\right] \frac{\partial E_{t}^{i i}}{\partial E_{t-1}^{i i}}+U_{c, t}^{i}\left[\frac{\partial C_{t}^{i}}{\partial E_{t}^{i j}}+\frac{U_{l, t}^{i}}{U_{c, t}^{i}} \frac{\partial l_{t}^{i}}{\partial E_{t}^{i j}}\right] \frac{\partial E_{t}^{i j}}{\partial E_{t-1}^{i i}}+} \\
U_{c, t}^{i} & {\left[\frac{\partial C_{t}^{i}}{\partial U_{t}^{i i}}+\frac{U_{l, t}^{i}}{U_{c, t}^{i}} \frac{\partial l_{t}^{i}}{\partial U_{t}^{i i}}\right] \frac{\partial U_{t}^{i i}}{\partial E_{t-1}^{i i}}+U_{c, t}^{i}\left[\frac{\partial C_{t}^{i}}{\partial U_{t}^{i j}}+\frac{U_{l, t}^{i}}{U_{c, t}^{i}} \frac{\partial l_{t}^{i}}{\partial U_{t}^{i j}}\right] \frac{\partial U_{t}^{i j}}{\partial E_{t-1}^{i i}}=}
\end{aligned}
$$




$$
\begin{gathered}
U_{c, t}^{i}(1-\rho)\left\{\left[\frac{W_{t}^{i i}}{P_{t}^{i}}-\frac{U_{l, t}^{i}}{U_{c, t}^{i}}\left(1-\alpha_{1}\right)^{1 / \nu_{1}}\left(\frac{E_{t}^{i i}+\zeta U_{t}^{i i}}{1-l_{t}^{i}}\right)^{-1 / \nu_{1}}\right]\left(1-f_{t}^{i i} \Omega_{t}^{i}\right)-\right. \\
{\left[\frac{W_{t}^{i j}}{P_{t}^{j}} Q_{t}^{i}-\frac{U_{l, t}^{i}}{U_{c, t}^{i}}\left(\alpha_{1}\right)^{1 / \nu_{1}}\left(\frac{E_{t}^{i j}+\zeta U_{t}^{i j}}{1-l_{t}^{i}}\right)^{-1 / \nu_{1}}\right] f_{t}^{i j}\left(1-\Omega_{t}^{i}\right)-} \\
{\left[b^{U}-\zeta \frac{U_{l, t}^{i}}{U_{c, t}^{i}}\left(1-\alpha_{1}\right)^{1 / \nu_{1}}\left(\frac{E_{t}^{i i}+\zeta U_{t}^{i i}}{1-l_{t}^{i}}\right)^{-1 / \nu_{1}}\right]\left(1-f_{t}^{i i}\right) \Omega_{t}^{i}-} \\
\left.\left[b^{U} Q_{t}^{i}-\zeta \frac{U_{l, t}^{i}}{U_{c, t}^{i}}\left(\alpha_{1}\right)^{1 / \nu_{1}}\left(\frac{E_{t}^{i j}+\zeta U_{t}^{i j}}{1-l_{t}^{i}}\right)^{-1 / \nu_{1}}\right]\left(1-f_{t}^{i i}\right)\left(1-\Omega_{t}^{i}\right)\right\} .
\end{gathered}
$$

The continuation value of being a native worker is the sum of the respective values for each of the five possible states in which a native worker in $t$ might end up in period $t+1$ :

1. employed as a native worker, gaining wage $W_{t+1}^{i i} / P_{t+1}^{i}$ and suffering disutility from working

2. unemployed searcher in labor market $i$, finding a new job in period $t\left(f_{t+1}^{i i} \Omega_{t+1}^{i}\right)$

3. unemployed searcher in labor market $i$, remaining unmatched at the end of $t$ $\left(\left(1-f_{t+1}^{i i}\right) \Omega_{t+1}^{i}\right)$

4. unemployed searcher in labor market $j$, finding a new job in period $t\left(f_{t+1}^{i j}(1-\right.$ $\left.\left.\Omega_{t+1}^{i}\right)\right)$

5. unemployed searcher in labor market $j$, remaining unmatched at the end of $t$ $\left(\left(1-f_{t+1}^{i j}\right)\left(1-\Omega_{t+1}^{i}\right)\right)$

The effect of an additional native worker on households' value function is given by ${ }^{18}$

$$
\frac{\partial \mathcal{V}_{t}^{i}}{\partial E_{t}^{i i}}=\frac{\partial \mathcal{U}_{t}^{i}}{\partial E_{t}^{i i}}+\beta \frac{\partial \mathcal{V}_{t+1}^{i}}{\partial E_{t}^{i i}}=0
$$

\footnotetext{
${ }^{18}$ Notice that the second equality in (A.11) follows from the fact

$$
\frac{\partial \mathcal{V}_{t}^{i}}{\partial E_{t-1}^{i i}}=\frac{\partial \mathcal{U}_{t}^{i}}{\partial E_{t-1}^{i i}}+\underbrace{\beta \frac{\partial \mathcal{V}_{t+1}^{i}}{\partial E_{t-1}^{i i}}}_{=0}=\frac{\partial \mathcal{U}_{t}^{i}}{\partial E_{t-1}^{i i}} .
$$
}




$$
\begin{array}{r}
\frac{\partial \mathcal{V}_{t}^{i}}{\partial E_{t}^{i i}}=U_{c, t}^{i}\left[\frac{W_{t}^{i i}}{P_{t}^{i}}-\frac{U_{l, t}^{i}}{U_{c, t}^{i}}\left(1-\alpha_{1}\right)^{1 / \nu_{1}}\left(\frac{E_{t}^{i i}+\zeta U_{t}^{i i}}{1-l_{t}^{i}}\right)^{-1 / \nu_{1}}\right]+\beta(1-\rho) U_{c, t+1}^{i} \\
\left\{\left(1-f_{t+1}^{i i} \Omega_{t+1}^{i}\right)\left[\frac{W_{t+1}^{i i}}{P_{t+1}^{i}}-\frac{U_{l, t+1}^{i}}{U_{c, t+1}^{i}}\left(1-\alpha_{1}\right)^{1 / \nu_{1}}\left(\frac{E_{t+1}^{i i}+\zeta U_{t+1}^{i i}}{1-l_{t+1}^{i}}\right)^{-1 / \nu_{1}}\right]-\right. \\
\left(1-\Omega_{t+1}^{i}\right) f_{t+1}^{i j}\left[\frac{W_{t+1}^{i j}}{P_{t+1}^{j}} Q_{t+1}^{i}-\frac{U_{l, t+1}^{i}}{U_{c, t+1}^{i}}\left(\alpha_{1}\right)^{1 / \nu_{1}}\left(\frac{E_{t+1}^{i j}+\zeta U_{t+1}^{i j}}{1-l_{t+1}^{i}}\right)^{-1 / \nu_{1}}\right]- \\
\Omega_{t+1}^{i}\left(1-f_{t+1}^{i i}\right)\left[b^{U}-\zeta \frac{U_{l, t+1}^{i}}{U_{c, t+1}^{i}}\left(1-\alpha_{1}\right)^{1 / \nu_{1}}\left(\frac{E_{t+1}^{i i}+\zeta U_{t+1}^{i i}}{1-l_{t+1}^{i}}\right)^{-1 / \nu_{1}}\right]- \\
\left.\left(1-\Omega_{t+1}^{i}\right)\left(1-f_{t+1}^{i j}\right)\left[b^{U} Q_{t+1}^{i}-\zeta \frac{U_{l, t+1}^{i}}{U_{c, t+1}^{i}}\left(\alpha_{1}\right)^{1 / \nu_{1}}\left(\frac{E_{t+1}^{i j}+\zeta U_{t+1}^{i j}}{1-l_{t+1}^{i}}\right)^{-1 / \nu_{1}}\right]\right\}
\end{array}
$$

The effect of one additional native unemployed on households' value function is

$$
\begin{aligned}
\frac{\partial \mathcal{V}_{t}^{i}}{\partial U_{t}^{i i}} & =\frac{\partial \mathcal{U}_{t}^{i}}{\partial U_{t}^{i i}}+\underbrace{\beta \frac{\partial \mathcal{V}_{t+1}^{i}}{\partial U_{t}^{i i}}}_{=0}=\frac{\partial \mathcal{U}_{t}^{i}}{\partial U_{t}^{i i}}=0 \\
& =U_{c, t}^{i}\left\{b^{U}-\zeta \frac{U_{l, t}^{i}}{U_{c, t}^{i}}\left(1-\alpha_{1}\right)^{1 / \nu_{1}}\left(\frac{E_{t}^{i i}+\zeta U_{t}^{i i}}{1-l_{t}^{i}}\right)^{-1 / \nu_{1}}\right\} .
\end{aligned}
$$

A native worker's surplus is then defined as the difference between the value of native employment and the value of native unemployment: ${ }^{19}$

$$
\bar{V}_{W, t}^{i i} \equiv \frac{\partial \mathcal{V}_{t}^{i}}{\partial E_{t}^{i i}}-\frac{\partial \mathcal{V}_{t}^{i}}{\partial U_{t}^{i i}}=\frac{\partial \mathcal{U}_{t}^{i}}{\partial E_{t}^{i i}}-\frac{\partial \mathcal{U}_{t}^{i}}{\partial U_{t}^{i i}}+\beta \frac{\partial \mathcal{V}_{t+1}^{i}}{\partial E_{t}^{i i}}=0
$$

\footnotetext{
${ }^{19}$ To be precise, the surplus of employing one additional native worker is computed as the difference between the value of native employment and the value of native unemployment, by keeping constant the fraction of searching members that are sent to labor markets $i$ and $j$, i.e. for $\Omega_{t}^{i}=\overline{\Omega^{i}}$. As one can show, the first-order conditions with respect to native and migrant (un)employment are, however, unaffected by fixing $\Omega_{t}^{i}=\overline{\Omega^{i}}$.
} 


$$
\begin{aligned}
& =U_{c, t}^{i}\left\{\frac{W_{t}^{i i}}{P_{t}^{i}}-b^{U}-(1-\zeta) \frac{U_{l, t}^{i}}{U_{c, t}^{i}}\left(1-\alpha_{1}\right)^{1 / \nu_{1}}\left(\frac{E_{t}^{i i}+\zeta U_{t}^{i i}}{1-l_{t}^{i}}\right)^{-1 / \nu_{1}}\right\}+ \\
& \beta(1-\rho) U_{c, t+1}^{i}\left\{\left[\frac{W_{t+1}^{i i}}{P_{t+1}^{i}}-\frac{U_{l, t+1}^{i}}{U_{c, t+1}^{i}}\left(1-\alpha_{1}\right)^{1 / \nu_{1}}\left(\frac{E_{t+1}^{i i}+\zeta U_{t+1}^{i i}}{1-l_{t+1}^{i}}\right)^{-1 / \nu_{1}}\right]-\right. \\
& \Omega_{t+1}^{i}{ }_{t+1}^{i i}\left[\frac{W_{t+1}^{i i}}{P_{t+1}^{i}}-\frac{U_{l, t+1}^{i}}{U_{c, t+1}^{i}}\left(1-\alpha_{1}\right)^{1 / \nu_{1}}\left(\frac{E_{t+1}^{i i}+\zeta U_{t+1}^{i i}}{1-l_{t+1}^{i}}\right)^{-1 / \nu_{1}}\right]- \\
& \Omega_{t+1}^{i}\left(1-f_{t+1}^{i i}\right)\left[b^{U}-\zeta \frac{U_{l, t+1}^{i}}{U_{c, t+1}^{i}}\left(1-\alpha_{1}\right)^{1 / \nu_{1}}\left(\frac{E_{t+1}^{i i}+\zeta U_{t+1}^{i i}}{1-l_{t+1}^{i}}\right)^{-1 / \nu_{1}}\right]- \\
& \left(1-\Omega_{t+1}^{i}\right) f_{t+1}^{i j}\left[\frac{W_{t+1}^{i j}}{P_{t+1}^{j}} Q_{t+1}^{i}-\frac{U_{l, t+1}^{i}}{U_{c, t+1}^{i}}\left(\alpha_{1}\right)^{1 / \nu_{1}}\left(\frac{E_{t+1}^{i j}+\zeta U_{t+1}^{i j}}{1-l_{t+1}^{i}}\right)^{-1 / \nu_{1}}\right]- \\
& \left.\left(1-\Omega_{t+1}^{i}\right)\left(1-f_{t+1}^{i j}\right)\left[b^{U} Q_{t+1}^{i}-\zeta \frac{U_{l, t+1}^{i}}{U_{c, t+1}^{i}}\left(\alpha_{1}\right)^{1 / \nu_{1}}\left(\frac{E_{t+1}^{i j}+\zeta U_{t+1}^{i j}}{1-l_{t+1}^{i}}\right)^{-1 / \nu_{1}}\right]\right\}=0 .
\end{aligned}
$$

In order to find a recursive expression of native workers' surplus, consider again the envelope condition for $E_{t}^{i i}$, equation (A.11):

$$
\begin{aligned}
& \frac{\partial \mathcal{V}_{t+1}^{i}}{\partial E_{t}^{i i}}= \frac{\partial \mathcal{V}_{t+1}^{i}}{\partial E_{t+1}^{i i}} \frac{\partial E_{t+1}^{i i}}{\partial E_{t}^{i i}}+\frac{\partial \mathcal{V}_{t+1}^{i}}{\partial U_{t+1}^{i i}} \frac{\partial U_{t+1}^{i i}}{\partial E_{t}^{i i}}+\frac{\partial \mathcal{V}_{t+1}^{i}}{\partial E_{t+1}^{i j}} \frac{\partial E_{t+1}^{i j}}{\partial E_{t}^{i i}}+\frac{\partial \mathcal{V}_{t+1}^{i}}{\partial U_{t+1}^{i j}} \frac{\partial U_{t+1}^{i j}}{\partial E_{t}^{i i}} \\
&= \frac{\partial \mathcal{V}_{t+1}^{i}}{\partial E_{t+1}^{i i}}(1-\rho)\left(1-f_{t+1}^{i i} \Omega_{t+1}^{i}\right)-\frac{\partial \mathcal{V}_{t+1}^{i}}{\partial U_{t+1}^{i i}}(1-\rho)\left(1-f_{t+1}^{i i}\right) \Omega_{t+1}^{i} \\
&- \frac{\partial \mathcal{V}_{t+1}^{i}}{\partial E_{t+1}^{i j}}(1-\rho) f_{t+1}^{i j}\left(1-\Omega_{t+1}^{i}\right)-\frac{\partial \mathcal{V}_{t+1}^{i}}{\partial U_{t+1}^{i j}}(1-\rho)\left(1-f_{t+1}^{i j}\right)\left(1-\Omega_{t+1}^{i}\right) \\
&=(1-\rho)\left\{\frac{\partial \mathcal{V}_{t+1}^{i}}{\partial E_{t+1}^{i i}}-\Omega_{t+1}^{i}\left[f_{t+1}^{i i} \frac{\partial \mathcal{V}_{t+1}^{i}}{\partial E_{t+1}^{i i}}+\left(1-f_{t+1}^{i i}\right) \frac{\partial \mathcal{V}_{t+1}^{i}}{\partial U_{t+1}^{i i}}\right]-\right. \\
&\left.\left(1-\Omega_{t+1}^{i}\right)\left[f_{t+1}^{i j} \frac{\partial \mathcal{V}_{t+1}^{i}}{\partial E_{t+1}^{i j}}+\left(1-f_{t+1}^{i j}\right) \frac{\partial \mathcal{V}_{t+1}^{i}}{\partial U_{t+1}^{i j}}\right]\right\} \\
&=(1-\rho)\left(1-f_{t+1}^{i i}\right)\left\{\frac{\partial \mathcal{V}_{t+1}^{i}}{\partial E_{t+1}^{i i}}-\frac{\partial \mathcal{V}_{t+1}^{i}}{\partial U_{t+1}^{i i}}\right\}=(1-\rho)\left(1-f_{t+1}^{i i}\right) \bar{V}_{W, t+1}^{i i},
\end{aligned}
$$

where the last line follows from the first-order condition with respect to $\Omega_{t}^{i}$, equation (A.9). More precisely,

$$
\frac{\partial \mathcal{V}_{t+1}^{i}}{\partial E_{t}^{i i}}=(1-\rho)\left(1-f_{t+1}^{i i}\right) \bar{V}_{W, t+1}^{i i} \Longleftrightarrow \Omega_{t}^{i}=\Omega_{t}^{i^{*}} .
$$


Therefore, in order to define the surplus of native employment recursively, we need to condition on households' optimal migration decision, such that

$$
\begin{gathered}
\bar{V}_{W, t}^{i i} \equiv \frac{\partial \mathcal{U}_{t}^{i}}{\partial E_{t}^{i i}}-\frac{\partial \mathcal{U}_{t}^{i}}{\partial U_{t}^{i i}}+\left.\beta \frac{\partial \mathcal{V}_{t+1}^{i}}{\partial E_{t}^{i i}}\right|_{\Omega_{t}^{i}=\Omega_{t}^{i *}} \\
\bar{V}_{W, t}^{i i}=U_{c, t}\left\{\frac{W_{t}^{i i}}{P_{t}^{i}}-b^{U}-(1-\zeta) \frac{U_{l, t}^{i}}{U_{c, t}^{i}}\left(1-\alpha_{1}\right)^{1 / \nu_{1}}\left(\frac{E_{t}^{i i}+\zeta U_{t}^{i i}}{1-l_{t}^{i}}\right)^{-1 / \nu_{1}}\right\}+\beta(1-\rho)\left(1-f_{t+1}^{i i}\right) \bar{V}_{W, t+1}^{i i} .
\end{gathered}
$$

Finally, define the surplus from native employment to household $i$ in terms of current consumption of final goods, $V_{W, t}^{i i}=\bar{V}_{W, t}^{i i} / U_{c, t}^{i}$, such that

$$
\begin{aligned}
V_{W, t}^{i i}= & \frac{W_{t}^{i i}}{P_{t}^{i}}-b^{U}-(1-\zeta) \frac{U_{l, t}^{i}}{U_{c, t}^{i}}\left(1-\alpha_{1}\right)^{1 / \nu_{1}}\left(\frac{E_{t}^{i i}+\zeta U_{t}^{i i}}{1-l_{t}^{i}}\right)^{-1 / \nu_{1}}+ \\
& (1-\rho) \mathbf{E}_{\mathbf{t}}\left\{\mathcal{Q}_{t, t+1}\left(1-f_{t+1}^{i i}\right) V_{W, t+1}^{i i}\right\}
\end{aligned}
$$

Equation (A.20) is a recursive expression of native workers' surplus, expressed in terms of the final consumption basket of household $i$ and conditional on household $i$ 's optimal migration decision. ${ }^{20}$

Appendix A.3. Value of migrant workers

Households' first-order condition with respect to $E_{t}^{i j}$ states as

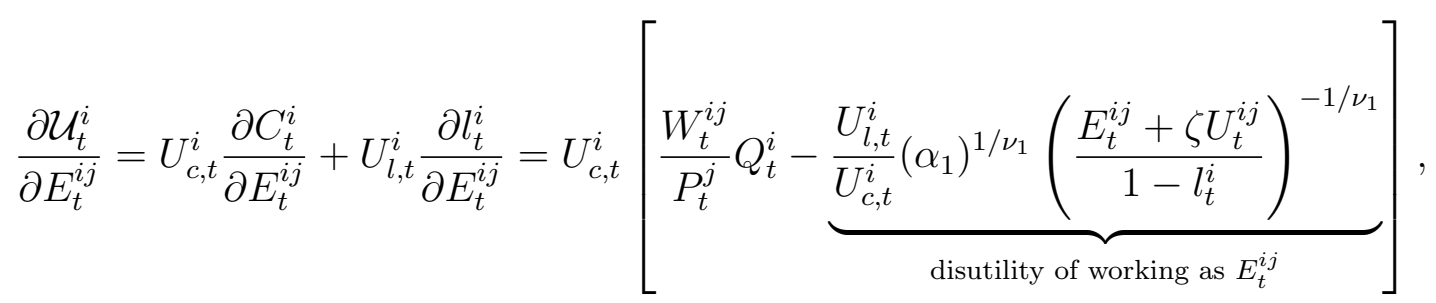

\footnotetext{
${ }^{20}$ The very same solution results from the problem where household $i$ takes the participation of its members in the foreign labor market, $L_{t}^{i j}$, as given. By choosing $\Omega_{t}^{i}$, the household decides upon the pool of searchers in both labor markets, $S_{t}^{i i}$ and $S_{t}^{i j}$, which directly defines $L_{t}^{i i}$ and $L_{t}^{i j}$. The search and matching process taking place in both labor markets, then defines the composition of both $L_{t}^{i i}=E_{t}^{i i}+U_{t}^{i i}$ and $L_{t}^{i j}=E_{t}^{i j}+U_{t}^{i j}$.
} 
and the first-order condition with respect to $E_{t-1}^{i j}$ states as

$$
\begin{aligned}
& \frac{\partial \mathcal{V}_{t}^{i}}{\partial E_{t-1}^{i j}}=\frac{\partial \mathcal{V}_{t}^{i}}{\partial E_{t}^{i j}} \frac{\partial E_{t}^{i j}}{\partial E_{t-1}^{i j}}+\frac{\partial \mathcal{V}_{t}^{i}}{\partial E_{t}^{i i}} \frac{\partial E_{t}^{i i}}{\partial E_{t-1}^{i j}}+\frac{\partial \mathcal{V}_{t}^{i}}{\partial U_{t}^{i j}} \frac{\partial U_{t}^{i j}}{\partial E_{t-1}^{i j}}+\frac{\partial \mathcal{V}_{t}^{i}}{\partial U_{t}^{i i}} \frac{\partial U_{t}^{i i}}{\partial E_{t-1}^{i j}}= \\
& \frac{\partial \mathcal{U}_{t}^{i}}{\partial E_{t}^{i j}} \frac{\partial E_{t}^{i j}}{\partial E_{t-1}^{i j}}+\frac{\partial \mathcal{U}_{t}^{i}}{\partial E_{t}^{i i}} \frac{\partial E_{t}^{i i}}{\partial E_{t-1}^{i j}}+\frac{\partial \mathcal{U}_{t}^{i}}{\partial U_{t}^{i j}} \frac{\partial U_{t}^{i j}}{\partial E_{t-1}^{i j}}+\frac{\partial \mathcal{U}_{t}^{i}}{\partial U_{t}^{i i}} \frac{\partial U_{t}^{i i}}{\partial E_{t-1}^{i j}}= \\
& U_{c, t}^{i}\left[\frac{\partial C_{t}^{i}}{\partial E_{t}^{i j}}+\frac{U_{l, t}^{i}}{U_{c, t}^{i}} \frac{\partial l_{t}^{i}}{\partial E_{t}^{i j}}\right] \frac{\partial E_{t}^{i j}}{\partial E_{t-1}^{i j}}+U_{c, t}^{i}\left[\frac{\partial C_{t}^{i}}{\partial E_{t}^{i i}}+\frac{U_{l, t}^{i}}{U_{c, t}^{i}} \frac{\partial l_{t}^{i}}{\partial E_{t}^{i i}}\right] \frac{\partial E_{t}^{i i}}{\partial E_{t-1}^{i j}}+ \\
& U_{c, t}^{i}\left[\frac{\partial C_{t}^{i}}{\partial U_{t}^{i j}}+\frac{U_{l, t}^{i}}{U_{c, t}^{i}} \frac{\partial l_{t}^{i}}{\partial U_{t}^{i j}}\right] \frac{\partial U_{t}^{i j}}{\partial E_{t-1}^{i j}}+U_{c, t}^{i}\left[\frac{\partial C_{t}^{i}}{\partial U_{t}^{i i}}+\frac{U_{l, t}^{i}}{U_{c, t}^{i}} \frac{\partial l_{t}^{i}}{\partial U_{t}^{i i}}\right] \frac{\partial U_{t}^{i i}}{\partial E_{t-1}^{i j}}= \\
& U_{c, t}^{i}(1-\rho)\left\{\left[\frac{W_{t}^{i j}}{P_{t}^{j}} Q_{t}^{i}-\frac{U_{l, t}^{i}}{U_{c, t}^{i}}\left(\alpha_{1}\right)^{1 / \nu_{1}}\left(\frac{E_{t}^{i j}+\zeta U_{t}^{i j}}{1-l_{t}^{i}}\right)^{-1 / \nu_{1}}\right]\left(1-f_{t}^{i j}\left(1-\Omega_{t}^{i}\right)\right)-\right. \\
& {\left[\frac{W_{t}^{i i}}{P_{t}^{i}}-\frac{U_{l, t}^{i}}{U_{c, t}^{i}}\left(1-\alpha_{1}\right)^{1 / \nu_{1}}\left(\frac{E_{t}^{i i}+\zeta U_{t}^{i i}}{1-l_{t}^{i}}\right)^{-1 / \nu_{1}}\right] f_{t}^{i i} \Omega_{t}^{i}-} \\
& {\left[b^{U} Q_{t}^{i}-\zeta \frac{U_{l, t}^{i}}{U_{c, t}^{i}}\left(\alpha_{1}\right)^{1 / \nu_{1}}\left(\frac{E_{t}^{i j}+\zeta U_{t}^{i j}}{1-l_{t}^{i}}\right)^{-1 / \nu_{1}}\right]\left(1-f_{t}^{i j}\right)\left(1-\Omega_{t}^{i}\right)-} \\
& \left.\left[b^{U}-\zeta \frac{U_{l, t}^{i}}{U_{c, t}^{i}}\left(1-\alpha_{1}\right)^{1 / \nu_{1}}\left(\frac{E_{t}^{i i}+\zeta U_{t}^{i i}}{1-l_{t}^{i}}\right)^{-1 / \nu_{1}}\right]\left(1-f_{t}^{i i}\right) \Omega_{t}^{i}\right\} .
\end{aligned}
$$

The effect of one additional migrant worker on households' value function is given by

$$
\frac{\partial \mathcal{V}_{t}^{i}}{\partial E_{t}^{i j}}=\frac{\partial \mathcal{U}_{t}^{i}}{\partial E_{t}^{i j}}+\beta \frac{\partial \mathcal{V}_{t+1}^{i}}{\partial E_{t}^{i j}}=0
$$




$$
\begin{gathered}
\frac{\partial \mathcal{V}_{t}^{i}}{\partial E_{t}^{i j}}=U_{c, t}^{i}\left[\frac{W_{t}^{i j}}{P_{t}^{j}} Q_{t}^{i}-\frac{U_{l, t}^{i}}{U_{c, t}^{i}}\left(\alpha_{1}\right)^{1 / \nu_{1}}\left(\frac{E_{t}^{i j}+\zeta U_{t}^{i j}}{1-l_{t}^{i}}\right)^{-1 / \nu_{1}}\right]+\beta(1-\rho) U_{c, t+1}^{i} \\
\left\{\left(1-f_{t+1}^{i j}\left(1-\Omega_{t+1}^{i}\right)\right)\left[\frac{W_{t+1}^{i j}}{P_{t+1}^{j} Q_{t+1}^{i}}-\frac{U_{l, t+1}^{i}}{U_{c, t+1}^{i}}\left(\alpha_{1}\right)^{1 / \nu_{1}}\left(\frac{E_{t+1}^{i j}+\zeta U_{t+1}^{i j}}{1-l_{t+1}^{i}}\right)^{-1 / \nu_{1}}\right]-\right. \\
\Omega_{t+1}^{i} f_{t+1}^{i i}\left[\frac{W_{t+1}^{i i}}{P_{t+1}^{i}}-\frac{U_{l, t+1}^{i}}{U_{c, t+1}^{i}}\left(1-\alpha_{1}\right)^{1 / \nu_{1}}\left(\frac{E_{t+1}^{i i}+\zeta U_{t+1}^{i i}}{1-l_{t+1}^{i}}\right)^{-1 / \nu_{1}}\right]- \\
\left(1-\Omega_{t+1}^{i}\right)\left(1-f_{t+1}^{i j}\right)\left[b^{U} Q_{t+1}^{i}-\zeta \frac{U_{l, t+1}^{i}}{U_{c, t+1}^{i}}\left(\alpha_{1}\right)^{1 / \nu_{1}}\left(\frac{E_{t+1}^{i j}+\zeta U_{t+1}^{i j}}{1-l_{t+1}^{i}}\right)^{-1 / \nu_{1}}\right]- \\
\left.\Omega_{t+1}^{i}\left(1-f_{t+1}^{i i}\right)\left[b^{U}-\zeta \frac{U_{l, t+1}^{i}}{U_{c, t+1}^{i}}\left(1-\alpha_{1}\right)^{1 / \nu_{1}}\left(\frac{E_{t+1}^{i i}+\zeta U_{t+1}^{i i}}{1-l_{t+1}^{i}}\right)^{-1 / \nu_{1}}\right]\right\} .
\end{gathered}
$$

The effect of one additional migrant unemployed on households' value function is

$$
\begin{aligned}
\frac{\partial \mathcal{V}_{t}^{i}}{\partial U_{t}^{i j}} & =\frac{\partial \mathcal{U}_{t}^{i}}{\partial U_{t}^{i j}}+\underbrace{\beta \frac{\partial \mathcal{V}_{t+1}^{i}}{\partial U_{t}^{i j}}}_{=0}=\frac{\partial \mathcal{U}_{t}^{i}}{\partial U_{t}^{i j}}=0 \\
& =U_{c, t}^{i}\left\{b^{U} Q_{t}^{i}-\zeta \frac{U_{l, t}^{i}}{U_{c, t}^{i}}\left(\alpha_{1}\right)^{1 / \nu_{1}}\left(\frac{E_{t}^{i j}+\zeta U_{t}^{i j}}{1-l_{t}^{i}}\right)^{-1 / \nu_{1}}\right\} .
\end{aligned}
$$

As for native workers, the surplus of migrant workers is computed as the difference between the value of migrant employment and migrant unemployment:

$$
\bar{V}_{W, t}^{i j} \equiv \frac{\partial \mathcal{V}_{t}^{i}}{\partial E_{t}^{i j}}-\frac{\partial \mathcal{V}_{t}^{i}}{\partial U_{t}^{i j}}=\frac{\partial \mathcal{U}_{t}^{i}}{\partial E_{t}^{i j}}+\frac{\partial \mathcal{U}_{t}^{i}}{\partial U_{t}^{i j}}+\beta \frac{\partial \mathcal{V}_{t+1}^{i}}{\partial E_{t}^{i j}}
$$




$$
\begin{gathered}
=U_{c, t}^{i}\left\{\frac{W_{t}^{i j}}{P_{t}^{j}} Q_{t}^{i}-b^{U} Q_{t}^{i}-(1-\zeta) \frac{U_{l, t}^{i}}{U_{c, t}^{i}}\left(\alpha_{1}\right)^{1 / \nu_{1}}\left(\frac{E_{t}^{i j}+\zeta U_{t}^{i j}}{1-l_{t}^{i}}\right)^{-1 / \nu_{1}}\right\}+ \\
\beta(1-\rho) U_{c, t+1}^{i}\left\{\left[\frac{W_{t+1}^{i j}}{P_{t+1}^{j}} Q_{t+1}^{i}-\frac{U_{l, t+1}^{i}}{U_{c, t+1}^{i}}\left(\alpha_{1}\right)^{1 / \nu_{1}}\left(\frac{E_{t+1}^{i j}+\zeta U_{t+1}^{i j}}{1-l_{t+1}^{i}}\right)^{-1 / \nu_{1}}\right]-\right. \\
\left(1-\Omega_{t+1}^{i}\right) f_{t+1}^{i j}\left[\frac{W_{t+1}^{i j}}{P_{t+1}^{j}} Q_{t+1}^{i}-\frac{U_{l, t+1}^{i}}{U_{c, t+1}^{i}}\left(\alpha_{1}\right)^{1 / \nu_{1}}\left(\frac{E_{t+1}^{i j}+\zeta U_{t+1}^{i j}}{1-l_{t+1}^{i}}\right)^{-1 / \nu_{1}}\right]- \\
\left(1-\Omega_{t+1}^{i}\right)\left(1-f_{t+1}^{i j}\right)\left[b^{U} Q_{t+1}^{i}-\zeta \frac{U_{l, t+1}^{i}}{U_{c, t+1}^{i}}\left(\alpha_{1}\right)^{1 / \nu_{1}}\left(\frac{E_{t+1}^{i j}+\zeta U_{t+1}^{i j}}{1-l_{t+1}^{i}}\right)^{-1 / \nu_{1}}\right]- \\
\Omega_{t+1}^{i} f_{t+1}^{i i}\left[\frac{\left.W_{t+1}^{i i}-\frac{U_{l, t+1}^{i}}{P_{t+1}^{i}}\left(1-\alpha_{1}\right)^{1 / \nu_{1}}\left(\frac{E_{t+1}^{i i}+\zeta U_{t+1}^{i i}}{1-l_{t+1}^{i}}\right)^{-1 / \nu_{1}}\right]+}{\left.U_{t+1}^{i}\left(1-f_{t+1}^{i i}\right)\left[b^{U}-\zeta \frac{U_{l, t+1}^{i}}{U_{c, t+1}^{i}}\left(1-\alpha_{1}\right)^{1 / \nu_{1}}\left(\frac{E_{t+1}^{i i}+\zeta U_{t+1}^{i i}}{1-l_{t+1}^{i}}\right)^{-1 / \nu_{1}}\right]\right\}}=0 .\right.
\end{gathered}
$$

As for native workers, conditional on the migration decision being optimal, the envelope condition for $E_{t}^{i j}$, equation (A.22), can be expressed in terms of the future surplus of migrant workers:

$$
\frac{\partial \mathcal{V}_{t+1}^{i}}{\partial E_{t}^{i j}}=(1-\rho)\left(1-f_{t+1}^{i j}\right) \bar{V}_{W, t+1}^{i j} \Longleftrightarrow \Omega_{t}^{i}=\Omega_{t}^{i^{*}}
$$

Therefore, in order to define the surplus of migrant employment recursively, we need to condition on households' optimal migration decision:

$$
\begin{gathered}
\bar{V}_{W, t}^{i j} \equiv \frac{\partial \mathcal{U}_{t}^{i}}{\partial E_{t}^{i j}}-\frac{\partial \mathcal{U}_{t}^{i}}{\partial U_{t}^{i j}}+\left.\beta \frac{\partial \mathcal{V}_{t+1}^{i}}{\partial E_{t}^{i j}}\right|_{\Omega_{t}^{i}=\Omega_{t}^{i *}} \\
\bar{V}_{W, t}^{i j}=U_{c, t}^{i}\left\{\frac{W_{t}^{i j}}{P_{t}^{j}} Q_{t}^{i}-b^{U} Q_{t}^{i}-(1-\zeta) \frac{U_{l, t}^{i}}{U_{c, t}^{i}}\left(\alpha_{1}\right)^{1 / \nu_{1}}\left(\frac{E_{t}^{i j}+\zeta U_{t}^{i j}}{1-l_{t}^{i}}\right)^{-1 / \nu_{1}}\right\}+\beta(1-\rho)\left(1-f_{t+1}^{i j}\right) \bar{V}_{W, t+1}^{i j} .
\end{gathered}
$$

Finally, define the surplus from migrant employment to household $i$ in terms of current consumption of final goods, $V_{W, t}^{i j}=\bar{V}_{W, t}^{i j} / U_{c, t}^{i}$, such that

$$
\begin{aligned}
V_{W, t}^{i j}= & \frac{W_{t}^{i j}}{P_{t}^{j}} Q_{t}^{i}-b^{U} Q_{t}^{i}-(1-\zeta) \frac{U_{l, t}^{i}}{U_{c, t}^{i}}\left(\alpha_{1}\right)^{1 / \nu_{1}}\left(\frac{E_{t}^{i j}+\zeta U_{t}^{i j}}{1-l_{t}^{i}}\right)^{-1 / \nu_{1}}+ \\
& (1-\rho) \mathbf{E}_{\mathbf{t}}\left\{\mathcal{Q}_{t, t+1}\left(1-f_{t+1}^{i j}\right) V_{W, t+1}^{i j}\right\} .
\end{aligned}
$$


Equation (A.29) is a recursive expression of migrant workers' surplus, expressed in terms of the final consumption basket of household $i$ and conditional on household $i$ 's optimal migration decision.

Appendix B. Equilibrium conditions benchmark model (fixed labor force)

$$
\begin{gathered}
\beta R_{t} \mathbf{E}_{\mathbf{t}}\left\{\left(\frac{U_{c, t+1}^{i}}{U_{c, t}^{i}}\right)\left(\frac{1}{\Pi_{t+1}^{i}}\right)\right\}=1 \\
U_{c, t}^{i} Q_{t}^{i}=U_{c, t}^{j} \vartheta \\
U_{c, t}^{i}=b\left(C_{t}^{i}\right)^{b(1-\sigma)-1}\left(l_{t}^{i}\right)^{(1-b)(1-\sigma)} Z_{t}^{i} \\
U_{c, t}^{j}=b\left(C_{t}^{j}\right)^{b(1-\sigma)-1}\left(l_{t}^{j}\right)^{(1-b)(1-\sigma)} Z_{t}^{j} \\
U_{l, t}^{i}=(1-b)\left(C_{t}^{i}\right)^{b(1-\sigma)}\left(l_{t}^{i}\right)^{(1-b)(1-\sigma)-1} \\
U_{l, t}^{j}=(1-b)\left(C_{t}^{j}\right)^{b(1-\sigma)}\left(l_{t}^{j}\right)^{(1-b)(1-\sigma)-1} \\
E_{t}^{i}=(1-\rho) E_{t-1}^{i}+f_{t}^{i} S_{t}^{i} \\
S_{t}^{j}=1-(1-\rho) E_{t-1}^{j} \\
E_{t}^{j}=(1-\rho) E_{t-1}^{j}+f_{t}^{j} S_{t}^{j} \\
\end{gathered}
$$




$$
\begin{aligned}
& 1=E_{t}^{i}+U_{t}^{i} \\
& 1=E_{t}^{j}+U_{t}^{j}
\end{aligned}
$$

$$
l_{t}^{i}=1-E_{t}^{i}-\zeta U_{t}^{i}
$$

$$
l_{t}^{j}=1-E_{t}^{j}-\zeta U_{t}^{j}
$$

$$
q_{t}^{i}=\tau_{t}^{i}\left(\theta_{t}^{i}\right)^{\gamma-1}
$$

$$
q_{t}^{j}=\tau_{t}^{j}\left(\theta_{t}^{j}\right)^{\gamma-1}
$$

$$
f_{t}^{i}=\tau_{t}^{i}\left(\theta_{t}^{i}\right)^{\gamma}
$$

$$
f_{t}^{j}=\tau_{t}^{j}\left(\theta_{t}^{j}\right)^{\gamma}
$$

$$
\theta_{t}^{i}=\frac{V_{t}^{i}}{S_{t}^{i}}
$$

$$
\theta_{t}^{j}=\frac{V_{t}^{j}}{S_{t}^{j}}
$$

$$
\begin{aligned}
& \frac{\kappa}{q_{t}^{i}} \frac{P_{H, t}^{i}}{P_{t}^{i}}=\frac{P_{x, t}^{i}}{P_{t}^{i}} A_{t}^{i}-\frac{W_{t}^{i}}{P_{t}^{i}}+(1-\rho) \mathbf{E}_{\mathbf{t}}\left\{\beta \frac{U_{c, t+1}^{i}}{U_{c, t}^{i}} \frac{\kappa}{q_{t+1}^{i}} \frac{P_{H, t+1}^{i}}{P_{t+1}^{i}}\right\} \\
& \frac{\kappa}{q_{t}^{j}} \frac{P_{H, t}^{j}}{P_{t}^{j}}=\frac{P_{x, t}^{j}}{P_{t}^{j}} A_{t}^{j}-\frac{W_{t}^{j}}{P_{t}^{j}}+(1-\rho) \mathbf{E}_{\mathbf{t}}\left\{\beta \frac{U_{c, t+1}^{j}}{U_{c, t}^{j}} \frac{\kappa}{q_{t+1}^{j}} \frac{P_{H, t+1}^{j}}{P_{t+1}^{j}}\right\}
\end{aligned}
$$




$$
\begin{aligned}
& \frac{W_{t}^{i}}{P_{t}^{i}}=\eta_{t}^{i}\left[b^{U}+(1-\zeta) \frac{U_{l, t}^{i}}{U_{c, t}^{i}}\right] \\
& +\left(1-\eta_{t}^{i}\right)\left[\frac{P_{x, t}^{i}}{P_{t}^{i}} A_{t}^{i}+(1-\rho) \mathbf{E}_{\mathbf{t}}\left\{\beta \frac{U_{c, t+1}^{i}}{U_{c, t}^{i}} \frac{\kappa}{q_{t+1}^{i}} f_{t+1}^{i} \frac{P_{H, t+1}^{i}}{P_{t+1}^{i}}\right\}\right] \\
& \frac{W_{t}^{j}}{P_{t}^{j}}=\eta_{t}^{i}\left[b^{U}+(1-\zeta) \frac{U_{l, t}^{j}}{U_{c, t}^{j}}\right] \\
& +\left(1-\eta_{t}^{i}\right)\left[\frac{P_{x, t}^{j}}{P_{t}^{j}} A_{t}^{j}+(1-\rho) \mathbf{E}_{\mathbf{t}}\left\{\beta \frac{U_{c, t+1}^{j}}{U_{c, t}^{j}} \frac{\kappa}{q_{t+1}^{j}} f_{t+1}^{j} \frac{P_{H, t+1}^{j}}{P_{t+1}^{j}}\right\}\right] \\
& X_{t}^{i}=A_{t}^{i} E_{t}^{i} \\
& X_{t}^{j}=A_{t}^{j} E_{t}^{j} \\
& R M C_{t}^{i}=\frac{P_{x, t}^{i}}{P_{t}^{i}} \underbrace{\left[\left(1-\alpha_{2}\right)+\alpha_{2} \frac{\left(1-\alpha_{2}\right)\left(Q_{t}^{i}\right)^{1-\nu_{2}}-\alpha_{2}}{\left(1-\alpha_{2}\right)-\alpha_{2}\left(Q_{t}^{i}\right)^{1-\nu_{2}}}\right]^{\frac{1}{1-\nu_{2}}}}_{=\frac{P_{t}^{i}}{P_{H, t}^{i}}} \frac{\left(Y_{t}^{i}\right)^{\frac{\alpha}{1-\alpha}}}{1-\alpha} \\
& R M C_{t}^{j}=\frac{P_{x, t}^{j}}{P_{t}^{j}} \underbrace{\left[\left(1-\alpha_{2}\right)+\alpha_{2} \frac{\left(1-\alpha_{2}\right)-\alpha_{2}\left(Q_{t}^{i}\right)^{1-\nu_{2}}}{\left(1-\alpha_{2}\right)\left(Q_{t}^{i}\right)^{1-\nu_{2}}-\alpha_{2}}\right]^{\frac{1}{1-\nu_{2}}}}_{\frac{P_{t}^{j}}{P_{H, t}^{j}}} \frac{\left(Y_{t}^{j}\right)^{\frac{\alpha}{1-\alpha}}}{1-\alpha} \\
& \frac{P_{H, t}^{i \star}}{P_{H, t}^{i}}=\left(\frac{F_{1, t}^{i}}{F_{2, t}^{i}}\right)^{\frac{1-\alpha}{1-\alpha+\varepsilon \alpha}}=\left(\frac{1-\delta\left(\Pi_{H, t}^{i}\right)^{\varepsilon-1}}{1-\delta}\right)^{\frac{1}{1-\varepsilon}} \\
& \frac{P_{H, t}^{j \star}}{P_{H, t}^{j}}=\left(\frac{F_{1, t}^{j}}{F_{2, t}^{j}}\right)^{\frac{1-\alpha}{1-\alpha+\varepsilon \alpha}}=\left(\frac{1-\delta\left(\Pi_{H, t}^{j}\right)^{\varepsilon-1}}{1-\delta}\right)^{\frac{1}{1-\varepsilon}} \\
& F_{1, t}^{i} \equiv \frac{1}{1-\varsigma} \frac{\varepsilon}{\varepsilon-1} U_{c, t}^{i} R M C_{t}^{i} \frac{P_{H, t}^{i}}{P_{t}^{i}} Y_{t}^{i}+\delta \beta \mathbf{E}_{t}\left\{\left(\Pi_{H, t+1}^{i}\right)^{\frac{\varepsilon}{1-\alpha}} F_{1, t+1}^{i}\right\}
\end{aligned}
$$




$$
\begin{aligned}
& F_{1, t}^{j} \equiv \frac{1}{1-\varsigma} \frac{\varepsilon}{\varepsilon-1} U_{c, t}^{j} R M C_{t}^{j} \frac{P_{H, t}^{j}}{P_{t}^{j}} Y_{t}^{j}+\delta \beta \mathbf{E}_{t}\left\{\left(\Pi_{H, t+1}^{j}\right)^{\frac{\varepsilon}{1-\alpha}} F_{1, t+1}^{j}\right\} \\
& F_{2, t}^{i} \equiv U_{c, t}^{i} \frac{P_{H, t}^{i}}{P_{t}^{i}} Y_{t}^{i}+\delta \beta \mathbf{E}_{t}\left\{\left(\Pi_{H, t+1}^{i}\right)^{\varepsilon-1} F_{2, t+1}^{i}\right\} \\
& F_{2, t}^{j} \equiv U_{c, t}^{j} \frac{P_{H, t}^{j}}{P_{t}^{j}} Y_{t}^{j}+\delta \beta \mathbf{E}_{t}\left\{\left(\Pi_{H, t+1}^{j}\right)^{\varepsilon-1} F_{2, t+1}^{j}\right\} \\
& \Delta_{t}^{i}=\delta\left(\Pi_{H, t}^{i}\right)^{\frac{\varepsilon}{1-\alpha}} \Delta_{t-1}^{i}+(1-\delta)\left(\frac{1-\delta\left(\Pi_{H, t}^{i}\right)^{\varepsilon-1}}{1-\delta}\right)^{\frac{\varepsilon}{(1-\varepsilon)(\alpha-1)}} \\
& \Delta_{t}^{j}=\delta\left(\Pi_{H, t}^{j}\right)^{\frac{\varepsilon}{1-\alpha}} \Delta_{t-1}^{j}+(1-\delta)\left(\frac{1-\delta\left(\Pi_{H, t}^{j}\right)^{\varepsilon-1}}{1-\delta}\right)^{\frac{\varepsilon}{(1-\varepsilon)(\alpha-1)}} \\
& Y_{t}^{i}=\left(X_{t}^{i}\right)^{1-\alpha}\left(\Delta_{t}^{i}\right)^{\alpha-1} \\
& Y_{t}^{j}=\left(X_{t}^{j}\right)^{1-\alpha}\left(\Delta_{t}^{j}\right)^{\alpha-1}
\end{aligned}
$$

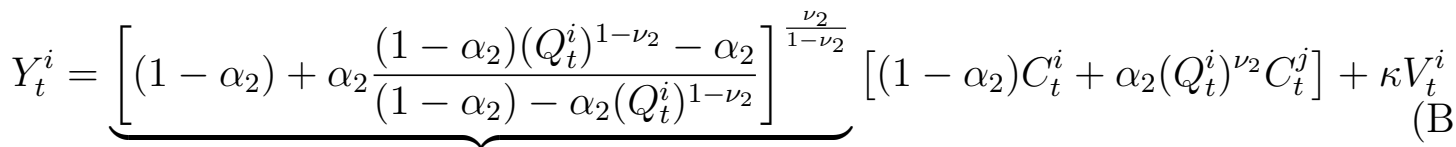

$$
\begin{aligned}
& \left(\frac{P_{H, t}^{i}}{P_{t}^{i}}\right)^{-\nu_{2}} \\
& Y_{t}^{j}=\underbrace{\left[\left(1-\alpha_{2}\right)+\alpha_{2} \frac{\left(1-\alpha_{2}\right)-\alpha_{2}\left(Q_{t}^{i}\right)^{1-\nu_{2}}}{\left(1-\alpha_{2}\right)\left(Q_{t}^{i}\right)^{1-\nu_{2}}-\alpha_{2}}\right]^{\frac{\nu_{2}}{1-\nu_{2}}}}_{\left(\frac{P_{H, t}^{j}}{P_{t}^{j}}\right)^{-\nu_{2}}}\left[\left(1-\alpha_{2}\right) C_{t}^{j}+\alpha_{2}\left(Q_{t}^{i}\right)^{-\nu_{2}} C_{t}^{i}\right]+\kappa V_{t}^{j} \\
& Q_{t}^{i}=\frac{\Pi_{t}^{j}}{\prod_{t}^{i}} Q_{t-1}^{i} \\
& \frac{\prod_{H, t}^{i}}{\prod_{t}^{i}}=\frac{\left[\left(1-\alpha_{2}\right)+\alpha_{2} \frac{\left(1-\alpha_{2}\right)\left(Q_{t}^{i}\right)^{1-\nu_{2}-\alpha_{2}}}{\left(1-\alpha_{2}\right)-\alpha_{2}\left(Q_{t}^{i}\right)^{1-\nu_{2}}}\right]^{\frac{1}{\nu_{2}-1}}}{\left[\left(1-\alpha_{2}\right)+\alpha_{2} \frac{\left(1-\alpha_{2}\right)\left(Q_{t-1}^{i}\right)^{1-\nu_{2}-\alpha_{2}}}{\left(1-\alpha_{2}\right)-\alpha_{2}\left(Q_{t-1}^{i}\right)^{1-\nu_{2}}}\right]^{\frac{1}{\nu_{2}-1}}}=\frac{P_{H, t}^{i} / P_{t}^{i}}{P_{H, t-1}^{i} / P_{t-1}^{i}}
\end{aligned}
$$




$$
\frac{\Pi_{H, t}^{j}}{\Pi_{t}^{j}}=\frac{\left[\left(1-\alpha_{2}\right)+\alpha_{2} \frac{\left(1-\alpha_{2}\right)-\alpha_{2}\left(Q_{t}^{i}\right)^{1-\nu_{2}}}{\left(1-\alpha_{2}\right)\left(Q_{t}^{i}\right)^{1-\nu_{2}-\alpha_{2}}}\right]^{\frac{1}{\nu_{2}-1}}}{\left[\left(1-\alpha_{2}\right)+\alpha_{2} \frac{\left(1-\alpha_{2}\right)-\alpha_{2}\left(Q_{t-1}^{i}\right)^{1-\nu_{2}}}{\left(1-\alpha_{2}\right)\left(Q_{t-1}^{i}\right)^{1-\nu_{2}-\alpha_{2}}}\right]^{\frac{1}{\nu_{2}-1}}}=\frac{P_{H, t}^{j} / P_{t}^{j}}{P_{H, t-1}^{j} / P_{t-1}^{j}}
$$

The model is closed with a definition of monetary policy and the law of motion for all three exogenous shocks.

\section{Appendix C. Equilibrium conditions full model with labor mobility}

$$
\begin{gathered}
\beta R_{t} \mathbf{E}_{\mathbf{t}}\left\{\left(\frac{U_{c, t+1}^{i}}{U_{c, t}^{i}}\right)\left(\frac{1}{\Pi_{t+1}^{i}}\right)\right\}=1 \\
U_{c, t}^{i} Q_{t}^{i}=U_{c, t}^{j} \vartheta \\
U_{c, t}^{i}=b\left(C_{t}^{i}\right)^{b(1-\sigma)-1}\left(l_{t}^{i}\right)^{(1-b)(1-\sigma)} Z_{t}^{i} \\
U_{c, t}^{j}=b\left(C_{t}^{j}\right)^{b(1-\sigma)-1}\left(l_{t}^{j}\right)^{(1-b)(1-\sigma)} Z_{t}^{j} \\
U_{l, t}^{i}=(1-b)\left(C_{t}^{i}\right)^{b(1-\sigma)}\left(l_{t}^{i}\right)^{(1-b)(1-\sigma)-1} \\
U_{l, t}^{j}=(1-b)\left(C_{t}^{j}\right)^{b(1-\sigma)}\left(l_{t}^{j}\right)^{(1-b)(1-\sigma)-1} \\
E_{t}^{j i}=(1-\rho) E_{t-1}^{j i}+f_{t}^{j i} S_{t}^{j i} \\
1-l_{t}^{j}=\left[\left(1-\alpha_{1}\right)^{1 / \nu_{1}}\left(E_{t}^{j j}+\zeta U_{t}^{j j}\right)^{\frac{\nu_{1}-1}{\nu_{1}}}+\left(\alpha_{1}\right)^{1 / \nu_{1}}\left(E_{t}^{j i}+\zeta U_{t}^{j i}\right)^{\frac{\nu_{1}-1}{\nu_{1}}}\right]^{\frac{\nu_{1}}{\nu_{1}-1}} \\
\left.\left(1-\alpha_{1}\right)^{1 / \nu_{1}}\left(E_{t}^{i i}+\zeta U_{t}^{i i}\right)^{\frac{\nu_{1}-1}{\nu_{1}}}+\left(\alpha_{1}\right)^{1 / \nu_{1}}\left(E_{t}^{i j}+\zeta U_{t}^{i j}\right)^{\frac{\nu_{1}-1}{\nu_{1}}}\right]^{\frac{\nu_{1}}{\nu_{1}-1}}
\end{gathered}
$$




$$
\begin{aligned}
& E_{t}^{j j}=(1-\rho) E_{t-1}^{j j}+f_{t}^{j j} S_{t}^{j j} \\
& E_{t}^{i j}=(1-\rho) E_{t-1}^{i j}+f_{t}^{i j} S_{t}^{i j} \\
& S_{t}^{i i}=\Omega_{t}^{i}\left[1-(1-\rho) E_{t-1}^{i i}-(1-\rho) E_{t-1}^{i j}\right] \\
& S_{t}^{i j}=\left(1-\Omega_{t}^{i}\right)\left[1-(1-\rho) E_{t-1}^{i i}-(1-\rho) E_{t-1}^{i j}\right] \\
& S_{t}^{j j}=\Omega_{t}^{j}\left[1-(1-\rho) E_{t-1}^{j j}-(1-\rho) E_{t-1}^{j i}\right] \\
& S_{t}^{j i}=\left(1-\Omega_{t}^{j}\right)\left[1-(1-\rho) E_{t-1}^{j j}-(1-\rho) E_{t-1}^{j i}\right] \\
& U_{t}^{i i}=\left(1-f_{t}^{i i}\right) \Omega_{t}^{i}\left[1-(1-\rho) E_{t-1}^{i i}-(1-\rho) E_{t-1}^{i j}\right] \\
& U_{t}^{j i}=\left(1-f_{t}^{j i}\right)\left(1-\Omega_{t}^{j}\right)\left[1-(1-\rho) E_{t-1}^{j j}-(1-\rho) E_{t-1}^{j i}\right] \\
& U_{t}^{j j}=\left(1-f_{t}^{j j}\right) \Omega_{t}^{j}\left[1-(1-\rho) E_{t-1}^{j j}-(1-\rho) E_{t-1}^{j i}\right] \\
& U_{t}^{i j}=\left(1-f_{t}^{i j}\right)\left(1-\Omega_{t}^{i}\right)\left[1-(1-\rho) E_{t-1}^{i i}-(1-\rho) E_{t-1}^{i j}\right] \\
& E_{t}^{i}=E_{t}^{i i}+E_{t}^{j i} \\
& E_{t}^{j}=E_{t}^{j j}+E_{t}^{i j} \\
& U_{t}^{i}=U_{t}^{i i}+U_{t}^{j i} \\
& U_{t}^{j}=U_{t}^{j j}+U_{t}^{i j} \\
& S_{t}^{i}=S_{t}^{i i}+S_{t}^{j i} \\
& S_{t}^{j}=S_{t}^{j j}+S_{t}^{i j} \\
& V_{t}^{i}=V_{t}^{i i}+V_{t}^{j i}
\end{aligned}
$$




$$
V_{t}^{j}=V_{t}^{j j}+V_{t}^{i j}
$$

Optimal migration decision of household $i$ (Foc w.r. to $\Omega_{t}^{i}$ )

$$
\begin{array}{r}
f_{t}^{i i}\left[\frac{W_{t}^{i i}}{P_{t}^{i}}-\frac{U_{l, t}^{i}}{U_{c, t}^{i}}\left(1-\alpha_{1}\right)^{1 / \nu_{1}}\left(\frac{E_{t}^{i i}+\zeta U_{t}^{i i}}{1-l_{t}^{i}}\right)^{-1 / \nu_{1}}\right]+ \\
\left(1-f_{t}^{i i}\right)\left[b^{U}-\zeta \frac{U_{l, t}^{i}}{U_{c, t}^{i}}\left(1-\alpha_{1}\right)^{1 / \nu_{1}}\left(\frac{E_{t}^{i i}+\zeta U_{t}^{i i}}{1-l_{t}^{i}}\right)^{-1 / \nu_{1}}\right]= \\
f_{t}^{i j}\left[\frac{W_{t}^{i j}}{P_{t}^{j}} Q_{t}^{i}-\frac{U_{l, t}^{i}}{U_{c, t}^{i}}\left(\alpha_{1}\right)^{1 / \nu_{1}}\left(\frac{E_{t}^{i j}+\zeta U_{t}^{i j}}{1-l_{t}^{i}}\right)^{-1 / \nu_{1}}\right]+ \\
\left(1-f_{t}^{i j}\right)\left[Q_{t}^{i} b^{U}-\zeta \frac{U_{l, t}^{i}}{U_{c, t}^{i}}\left(\alpha_{1}\right)^{1 / \nu_{1}}\left(\frac{E_{t}^{i j}+\zeta U_{t}^{i j}}{1-l_{t}^{i}}\right)^{-1 / \nu_{1}}\right]
\end{array}
$$

Optimal migration decision of household $j$ (Foc w.r. to $\Omega_{t}^{j}$ )

$$
\begin{gathered}
f_{t}^{j j}\left[\frac{W_{t}^{j j}}{P_{t}^{j}}-\frac{U_{l, t}^{j}}{U_{c, t}^{j}}\left(1-\alpha_{1}\right)^{1 / \nu_{1}}\left(\frac{E_{t}^{j j}+\zeta U_{t}^{j j}}{1-l_{t}^{j}}\right)^{-1 / \nu_{1}}\right]+ \\
\left(1-f_{t}^{j j}\right)\left[b^{U}-\zeta \frac{U_{l, t}^{j}}{U_{c, t}^{j}}\left(1-\alpha_{1}\right)^{1 / \nu_{1}}\left(\frac{E_{t}^{j j}+\zeta U_{t}^{j j}}{1-l_{t}^{j}}\right)^{-1 / \nu_{1}}\right]= \\
f_{t}^{j i}\left[\frac{W_{t}^{j i}}{P_{t}^{i} Q_{t}^{i}}-\frac{U_{l, t}^{j}}{U_{c, t}^{j}}\left(\alpha_{1}\right)^{1 / \nu_{1}}\left(\frac{E_{t}^{j i}+\zeta U_{t}^{j i}}{1-l_{t}^{j}}\right)^{-1 / \nu_{1}}\right]+ \\
\left(1-f_{t}^{j i}\right)\left[\frac{b^{U}}{Q_{t}^{i}}-\zeta \frac{U_{l, t}^{j}}{U_{c, t}^{j}}\left(\alpha_{1}\right)^{1 / \nu_{1}}\left(\frac{E_{t}^{j i}+\zeta U_{t}^{j i}}{1-l_{t}^{j}}\right)^{-1 / \nu_{1}}\right] \\
q_{t}^{i i}=\tau_{t}^{i}\left(\theta_{t}^{i i}\right)^{\gamma-1} \\
q_{t}^{j i}=\tau_{t}^{i}\left(\theta_{t}^{j i}\right)^{\gamma-1} \\
q_{t}^{j j}=\tau_{t}^{j}\left(\theta_{t}^{j j}\right)^{\gamma-1} \\
q_{t}^{i j}=\tau_{t}^{j}\left(\theta_{t}^{i j}\right)^{\gamma-1} \\
f_{t}^{i i}=\tau_{t}^{i}\left(\theta_{t}^{i i}\right)^{\gamma}
\end{gathered}
$$




$$
\begin{gathered}
f_{t}^{j i}=\tau_{t}^{i}\left(\theta_{t}^{j i}\right)^{\gamma} \\
f_{t}^{j j}=\tau_{t}^{j}\left(\theta_{t}^{j j}\right)^{\gamma} \\
f_{t}^{i j}=\tau_{t}^{j}\left(\theta_{t}^{i j}\right)^{\gamma} \\
\theta_{t}^{i}=\frac{V_{t}^{i}}{S_{t}^{i}} \\
\theta_{t}^{j}=\frac{V_{t}^{j}}{S_{t}^{j}} \\
\theta_{t}^{i i}=\frac{V_{t}^{i i}}{S_{t}^{i i}} \\
\theta_{t}^{j i}=\frac{V_{t}^{j i}}{S_{t}^{j i}} \\
\theta_{t}^{j j}=\frac{V_{t}^{j j}}{S_{t}^{j j}} \\
\theta_{t}^{i j}=\frac{V_{t}^{i j}}{S_{t}^{i j}}
\end{gathered}
$$

Native job-creation condition in region $i$

$$
\frac{\kappa}{q_{t}^{i i}} \frac{P_{H, t}^{i}}{P_{t}^{i}}=\frac{P_{x, t}^{i}}{P_{t}^{i}} A_{t}^{i}-\frac{W_{t}^{i i}}{P_{t}^{i}}+(1-\rho) \mathbf{E}_{\mathbf{t}}\left\{\beta \frac{U_{c, t+1}^{i}}{U_{c, t}^{i}} \frac{\kappa}{q_{t+1}^{i i}} \frac{P_{H, t+1}^{i}}{P_{t+1}^{i}}\right\}
$$

Migrant job-creation condition in region $i$

$$
\frac{\kappa}{q_{t}^{j i}} \frac{P_{H, t}^{i}}{P_{t}^{i}}=\frac{P_{x, t}^{i}}{P_{t}^{i}} A_{t}^{i}-\frac{W_{t}^{j i}}{P_{t}^{i}}+(1-\rho) \mathbf{E}_{\mathbf{t}}\left\{\beta \frac{U_{c, t+1}^{j}}{U_{c, t}^{j}} \frac{\kappa}{q_{t+1}^{j i}} \frac{P_{H, t+1}^{i}}{P_{t+1}^{i}}\right\}
$$

Native job-creation condition in region $j$

$$
\frac{\kappa}{q_{t}^{j j}} \frac{P_{H, t}^{j}}{P_{t}^{j}}=\frac{P_{x, t}^{j}}{P_{t}^{j}} A_{t}^{j}-\frac{W_{t}^{j j}}{P_{t}^{j}}+(1-\rho) \mathbf{E}_{\mathbf{t}}\left\{\beta \frac{U_{c, t+1}^{j}}{U_{c, t}^{j}} \frac{\kappa}{q_{t+1}^{j j}} \frac{P_{H, t+1}^{j}}{P_{t+1}^{j}}\right\}
$$

Migrant job-creation condition in region $j$

$$
\frac{\kappa}{q_{t}^{i j}} \frac{P_{H, t}^{j}}{P_{t}^{j}}=\frac{P_{x, t}^{j}}{P_{t}^{j}} A_{t}^{j}-\frac{W_{t}^{i j}}{P_{t}^{j}}+(1-\rho) \mathbf{E}_{\mathbf{t}}\left\{\beta \frac{U_{c, t+1}^{i}}{U_{c, t}^{i}} \frac{\kappa}{q_{t+1}^{i j}} \frac{P_{H, t+1}^{j}}{P_{t+1}^{j}}\right\}
$$


Wage equations for native workers in region $i$

$$
\begin{aligned}
& \frac{W_{t}^{i i}}{P_{t}^{i}}=\eta_{t}^{i}\left[b^{U}+(1-\zeta) \frac{U_{l, t}^{i}}{U_{c, t}^{i}}\left(1-\alpha_{1}\right)^{1 / \nu_{1}}\left(\frac{E_{t}^{i i}+\zeta U_{t}^{i i}}{1-l_{t}^{i}}\right)^{-1 / \nu_{1}}\right] \\
& +\left(1-\eta_{t}^{i}\right)\left[\frac{P_{x, t}^{i}}{P_{t}^{i}} A_{t}^{i}+(1-\rho) \beta \mathbf{E}_{\mathbf{t}}\left\{\frac{U_{c, t+1}^{i}}{U_{c, t}^{i}} \frac{\kappa}{q_{t+1}^{i i}} f_{t+1}^{i i} \frac{P_{H, t+1}^{i}}{P_{t+1}^{i}}\right\}\right]
\end{aligned}
$$

Wage equations for migrant workers in region $i$

$$
\begin{aligned}
& \frac{W_{t}^{j i}}{P_{t}^{i}}=\left[\frac{\eta_{t}^{i}}{\eta_{t}^{i}+Q_{t}^{i}\left(1-\eta_{t}^{i}\right)}\right]\left[b^{U}+Q_{t}^{i}(1-\zeta) \frac{U_{l, t}^{j}}{U_{c, t}^{j}}\left(\alpha_{1}\right)^{1 / \nu_{1}}\left(\frac{E_{t}^{j i}+\zeta U_{t}^{j i}}{1-l_{t}^{j}}\right)^{-1 / \nu_{1}}\right] \\
& +\left[\frac{Q_{t}^{i}\left(1-\eta_{t}^{i}\right)}{\eta_{t}^{i}+Q_{t}^{i}\left(1-\eta_{t}^{i}\right)}\right]\left[\frac{P_{x, t}^{i}}{P_{t}^{i}} A_{t}^{i}+(1-\rho) \beta \mathbf{E}_{\mathbf{t}}\left\{\frac{U_{c, t+1}^{i}}{U_{c, t}^{i}} \frac{\kappa}{q_{t+1}^{j i}} \frac{P_{H, t+1}^{i}}{P_{t+1}^{i}}\left(1-\frac{Q_{t+1}^{i}}{Q_{t}^{i}}\left(1-f_{t+1}^{j i}\right)\right)\right\}\right]
\end{aligned}
$$

Wage equation for native workers in region $j$

$$
\begin{aligned}
& \frac{W_{t}^{j j}}{P_{t}^{j}}=\eta_{t}^{j}\left[b^{U}+(1-\zeta) \frac{U_{l, t}^{j}}{U_{c, t}^{j}}\left(1-\alpha_{1}\right)^{1 / \nu_{1}}\left(\frac{E_{t}^{j j}+\zeta U_{t}^{j j}}{1-l_{t}^{j}}\right)^{-1 / \nu_{1}}\right] \\
& \quad+\left(1-\eta_{t}^{j}\right)\left[\frac{P_{x, t}^{j}}{P_{t}^{j}} A_{t}^{j}+(1-\rho) \beta \mathbf{E}_{\mathbf{t}}\left\{\frac{U_{c, t+1}^{j}}{U_{c, t}^{j}} \frac{\kappa}{q_{t+1}^{j j}} f_{t+1}^{j j} \frac{P_{H, t+1}^{j}}{P_{t+1}^{j}}\right\}\right]
\end{aligned}
$$

Wage equation for migrant workers in region $j$

$$
\begin{gathered}
\frac{W_{t}^{i j}}{P_{t}^{j}}=\left[\frac{\eta_{t}^{j} Q_{t}^{i}}{\eta_{t}^{j} Q_{t}^{i}+\left(1-\eta_{t}^{j}\right)}\right]\left[b^{U}+\frac{1}{Q_{t}^{i}}(1-\zeta) \frac{U_{l, t}^{i}}{U_{c, t}^{i}}\left(\alpha_{1}\right)^{1 / \nu_{1}}\left(\frac{E_{t}^{i j}+\zeta U_{t}^{i j}}{1-l_{t}^{i}}\right)^{-1 / \nu_{1}}\right] \\
+\left[\frac{\left(1-\eta_{t}^{j}\right)}{\eta_{t}^{j} Q_{t}^{i}+\left(1-\eta_{t}^{j}\right)}\right]\left[\frac{P_{x, t}^{j}}{P_{t}^{j}} A_{t}^{j}+(1-\rho) \beta \mathbf{E}_{\mathbf{t}}\left\{\frac{U_{c, t+1}^{j}}{U_{c, t}^{j}} \frac{\kappa}{q_{t+1}^{i j}} \frac{P_{H, t+1}^{j}}{P_{t+1}^{j}}\left(1-\frac{Q_{t}^{i}}{Q_{t+1}^{i}}\left(1-f_{t+1}^{i j}\right)\right)\right\}\right] \\
X_{t}^{i}=A_{t}^{i} E_{t}^{i} \\
X_{t}^{j}=A_{t}^{j} E_{t}^{j} \\
R M C_{t}^{i}=\frac{P_{x, t}^{i}}{P_{t}^{i}} \underbrace{(\mathrm{C} .52}_{=\frac{P_{t}^{i}}{P_{H, t}^{i}}}
\end{gathered}
$$




$$
\begin{aligned}
& R M C_{t}^{j}=\frac{P_{x, t}^{j}}{P_{t}^{j}} \underbrace{\left[\left(1-\alpha_{2}\right)+\alpha_{2} \frac{\left(1-\alpha_{2}\right)-\alpha_{2}\left(Q_{t}^{i}\right)^{1-\nu_{2}}}{\left(1-\alpha_{2}\right)\left(Q_{t}^{i}\right)^{1-\nu_{2}}-\alpha_{2}}\right]^{\frac{1}{1-\nu_{2}}}}_{\frac{P_{t}^{j}}{P_{H, t}^{j}}} \frac{\left(Y_{t}^{j}\right)^{\frac{\alpha}{1-\alpha}}}{1-\alpha} \\
& \frac{P_{H, t}^{i \star}}{P_{H, t}^{i}}=\left(\frac{F_{1, t}^{i}}{F_{2, t}^{i}}\right)^{\frac{1-\alpha}{1-\alpha+\varepsilon \alpha}}=\left(\frac{1-\delta\left(\Pi_{H, t}^{i}\right)^{\varepsilon-1}}{1-\delta}\right)^{\frac{1}{1-\varepsilon}} \\
& \frac{P_{H, t}^{j \star}}{P_{H, t}^{j}}=\left(\frac{F_{1, t}^{j}}{F_{2, t}^{j}}\right)^{\frac{1-\alpha}{1-\alpha+\varepsilon \alpha}}=\left(\frac{1-\delta\left(\Pi_{H, t}^{j}\right)^{\varepsilon-1}}{1-\delta}\right)^{\frac{1}{1-\varepsilon}} \\
& F_{1, t}^{i} \equiv \frac{1}{1-\varsigma} \frac{\varepsilon}{\varepsilon-1} U_{c, t}^{i} R M C_{t}^{i} \frac{P_{H, t}^{i}}{P_{t}^{i}} Y_{t}^{i}+\delta \beta \mathbf{E}_{t}\left\{\left(\Pi_{H, t+1}^{i}\right)^{\frac{\varepsilon}{1-\alpha}} F_{1, t+1}^{i}\right\} \\
& F_{1, t}^{j} \equiv \frac{1}{1-\varsigma} \frac{\varepsilon}{\varepsilon-1} U_{c, t}^{j} R M C_{t}^{j} \frac{P_{H, t}^{j}}{P_{t}^{j}} Y_{t}^{j}+\delta \beta \mathbf{E}_{t}\left\{\left(\Pi_{H, t+1}^{j}\right)^{\frac{\varepsilon}{1-\alpha}} F_{1, t+1}^{j}\right\} \\
& F_{2, t}^{i} \equiv U_{c, t}^{i} \frac{P_{H, t}^{i}}{P_{t}^{i}} Y_{t}^{i}+\delta \beta \mathbf{E}_{t}\left\{\left(\Pi_{H, t+1}^{i}\right)^{\varepsilon-1} F_{2, t+1}^{i}\right\} \\
& F_{2, t}^{j} \equiv U_{c, t}^{j} \frac{P_{H, t}^{j}}{P_{t}^{j}} Y_{t}^{j}+\delta \beta \mathbf{E}_{t}\left\{\left(\Pi_{H, t+1}^{j}\right)^{\varepsilon-1} F_{2, t+1}^{j}\right\} \\
& \Delta_{t}^{i}=\delta\left(\Pi_{H, t}^{i}\right)^{\frac{\varepsilon}{1-\alpha}} \Delta_{t-1}^{i}+(1-\delta)\left(\frac{1-\delta\left(\Pi_{H, t}^{i}\right)^{\varepsilon-1}}{1-\delta}\right)^{\frac{\varepsilon}{(1-\varepsilon)(\alpha-1)}} \\
& \Delta_{t}^{j}=\delta\left(\Pi_{H, t}^{j}\right)^{\frac{\varepsilon}{1-\alpha}} \Delta_{t-1}^{j}+(1-\delta)\left(\frac{1-\delta\left(\Pi_{H, t}^{j}\right)^{\varepsilon-1}}{1-\delta}\right)^{\frac{\varepsilon}{(1-\varepsilon)(\alpha-1)}} \\
& Y_{t}^{i}=\left(X_{t}^{i}\right)^{1-\alpha}\left(\Delta_{t}^{i}\right)^{\alpha-1} \\
& Y_{t}^{j}=\left(X_{t}^{j}\right)^{1-\alpha}\left(\Delta_{t}^{j}\right)^{\alpha-1} \\
& Y_{t}^{i}=\underbrace{\left[\left(1-\alpha_{2}\right)+\alpha_{2} \frac{\left(1-\alpha_{2}\right)\left(Q_{t}^{i}\right)^{1-\nu_{2}}-\alpha_{2}}{\left(1-\alpha_{2}\right)-\alpha_{2}\left(Q_{t}^{i}\right)^{1-\nu_{2}}}\right]^{\frac{\nu_{2}}{1-\nu_{2}}}}_{\left(\frac{P_{H, t}^{i}}{P_{t}^{i}}\right)^{-\nu_{2}}}\left[\left(1-\alpha_{2}\right) C_{t}^{i}+\alpha_{2}\left(Q_{t}^{i}\right)^{\nu_{2}} C_{t}^{j}\right]+\kappa V_{t}^{i}
\end{aligned}
$$




$$
Y_{t}^{j}=\underbrace{\left[\left(1-\alpha_{2}\right)+\alpha_{2} \frac{\left(1-\alpha_{2}\right)-\alpha_{2}\left(Q_{t}^{i}\right)^{1-\nu_{2}}}{\left(1-\alpha_{2}\right)\left(Q_{t}^{i}\right)^{1-\nu_{2}}-\alpha_{2}}\right]^{\frac{\nu_{2}}{1-\nu_{2}}}}_{\left(\frac{P_{H, t}^{j}}{P_{t}^{j}}\right)^{-\nu_{2}}}\left[\left(1-\alpha_{2}\right) C_{t}^{j}+\alpha_{2}\left(Q_{t}^{i}\right)^{-\nu_{2}} C_{t}^{i}\right]+\kappa V_{t}^{j}
$$

$$
\begin{gathered}
Q_{t}^{i}=\frac{\Pi_{t}^{j}}{\prod_{t}^{i}} Q_{t-1}^{i} \\
\frac{\Pi_{H, t}^{i}}{\prod_{t}^{i}}=\frac{\left[\left(1-\alpha_{2}\right)+\alpha_{2} \frac{\left(1-\alpha_{2}\right)\left(Q_{t}^{i}\right)^{1-\nu_{2}-\alpha_{2}}}{\left(1-\alpha_{2}\right)-\alpha_{2}\left(Q_{t}^{i}\right)^{1-\nu_{2}}}\right]^{\frac{1}{\nu_{2}-1}}}{\left[\left(1-\alpha_{2}\right)+\alpha_{2} \frac{\left(1-\alpha_{2}\right)\left(Q_{t-1}^{i}\right)^{1-\nu_{2}-\alpha_{2}}}{\left(1-\alpha_{2}\right)-\alpha_{2}\left(Q_{t-1}^{i}\right)^{1-\nu_{2}}}\right]^{\frac{1}{\nu_{2}-1}}}=\frac{P_{H, t}^{i} / P_{t}^{i}}{P_{H, t-1}^{i} / P_{t-1}^{i}} \\
\frac{\Pi_{H, t}^{j}}{\Pi_{t}^{j}}=\frac{\left[\left(1-\alpha_{2}\right)+\alpha_{2} \frac{\left(1-\alpha_{2}\right)-\alpha_{2}\left(Q_{t}^{i}\right)^{1-\nu_{2}}}{\left(1-\alpha_{2}\right)\left(Q_{t}^{i}\right)^{1-\nu_{2}-\alpha_{2}}}\right]^{\frac{1}{\nu_{2}-1}}}{\left[\left(1-\alpha_{2}\right)+\alpha_{2} \frac{\left(1-\alpha_{2}\right)-\alpha_{2}\left(Q_{t-1}^{i}\right)^{1-\nu_{2}}}{\left(1-\alpha_{2}\right)\left(Q_{t-1}^{i}\right)^{1-\nu_{2}-\alpha_{2}}}\right]^{\frac{1}{\nu_{2}-1}}}=\frac{P_{H, t}^{j} / P_{t}^{j}}{P_{H, t-1}^{j} / P_{t-1}^{j}}
\end{gathered}
$$

The model is closed with a definition of monetary policy and the law of motion for all three exogenous shocks.

\section{Appendix D. Efficient allocation}

The cooperative social planner thus maximizes

$$
\mathbf{E}_{\mathbf{0}} \sum_{t=0}^{\infty} \beta^{t}\left[\mathcal{U}_{t}^{i}\left(C_{t}^{i}, l_{t}^{i}\right)+\mathcal{U}_{t}^{j}\left(C_{t}^{j}, l_{t}^{j}\right)\right]
$$


subject to

$$
\begin{aligned}
C_{t}^{i}= & \left\{\left(-\alpha_{2}\right)^{\frac{1}{\nu_{2}}}\left(C_{H, t}^{i}\right)^{\frac{\nu_{2}-1}{\nu_{2}}}+\left(\alpha_{2}\right)^{\frac{1}{\nu_{2}}}\left(C_{F, t}^{i}\right)^{\frac{\nu_{2}-1}{\nu_{2}}}\right\}^{\frac{\nu_{2}}{\nu_{2}-1}} \\
C_{t}^{j}= & \left\{\left(1-\alpha_{2}\right)^{\frac{1}{\nu_{2}}}\left(C_{H, t}^{j}\right)^{\frac{\nu_{2}-1}{\nu_{2}}}+\left(\alpha_{2}\right)^{\frac{1}{\nu_{2}}}\left(C_{F, t}^{j}\right)^{\frac{\nu_{2}-1}{\nu_{2}}}\right\}^{\frac{\nu_{2}}{\nu_{2}-1}} \\
l_{t}^{i}= & 1-\left[\left(1-\alpha_{1}\right)^{1 / \nu_{1}}\left(E_{t}^{i i}+\zeta U_{t}^{i i}\right)^{\frac{\nu_{1}-1}{\nu_{1}}}+\left(\alpha_{1}\right)^{1 / \nu_{1}}\left(E_{t}^{i j}+\zeta U_{t}^{i j}\right)^{\frac{\nu_{1}-1}{\nu_{1}}}\right]^{\frac{\nu_{1}}{\nu_{1}-1}} \\
l_{t}^{j j}= & 1-\left[\left(1-\alpha_{1}\right)^{1 / \nu_{1}}\left(E_{t}^{j j}+\zeta U_{t}^{j j}\right)^{\frac{\nu_{1}-1}{\nu_{1}}}+\left(\alpha_{1}\right)^{1 / \nu_{1}}\left(E_{t}^{j i}+\zeta U_{t}^{j i}\right)^{\frac{\nu_{1}-1}{\nu_{1}}}\right]^{\frac{\nu_{1}}{\nu_{1}-1}} \\
E_{t}^{i i}= & (1-\rho) E_{t-1}^{i i}+\tau_{t}^{i}\left(\theta_{t}^{i i}\right)^{\gamma} S_{t}^{i i} \\
E_{t}^{j i}= & (1-\rho) E_{t-1}^{j i}+\tau_{t}^{i}\left(\theta_{t}^{j i}\right)^{\gamma} S_{t}^{j i} \\
E_{t}^{j j}= & (1-\rho) E_{t-1}^{j j}+\tau_{t}^{j}\left(\theta_{t}^{j j}\right)^{\gamma} S_{t}^{j j} \\
E_{t}^{i j}= & (1-\rho) E_{t-1}^{i j}+\tau_{t}^{j}\left(\theta_{t}^{i j}\right)^{\gamma} S_{t}^{i j} \\
U_{t}^{i i}= & {\left[1-\tau_{t}^{i}\left(\theta_{t}^{i i}\right)^{\gamma}\right] S_{t}^{i i} } \\
U_{t}^{j i}= & {\left[1-\tau_{t}^{i}\left(\theta_{t}^{j i}\right)^{\gamma}\right] S_{t}^{j i} } \\
U_{t}^{j j}= & {\left[1-\tau_{t}^{j}\left(\theta_{t}^{j j}\right)^{\gamma}\right] S_{t}^{j j} } \\
U_{t}^{i j}= & {\left[1-\tau_{t}^{j}\left(\theta_{t}^{i j}\right)^{\gamma}\right] S_{t}^{i j} . } \\
& {\left[A_{t}^{i}\left(E_{t}^{i i}+E_{t}^{j i}\right)\right]^{(1-\alpha)}=C_{H, t}^{i}+C_{F, t}^{j}+\kappa \theta_{t}^{i i} S_{t}^{i i}+\kappa \theta_{t}^{j i} S_{t}^{j i} } \\
& {\left[A_{t}^{j}\left(E_{t}^{j j}+E_{t}^{i j}\right)\right]^{(1-\alpha)}=C_{H, t}^{j}+C_{F, t}^{i}+\kappa \theta_{t}^{j j} S_{t}^{j j}+\kappa \theta_{t}^{i j} S_{t}^{i j} }
\end{aligned}
$$

Notice that the sum of constraints (D.6), (D.9), (D.10), and (D.13) yields

$$
\begin{aligned}
E_{t}^{i i}+E_{t}^{i j}+U_{t}^{i i}+U_{t}^{i j} & = \\
(1-\rho) E_{t-1}^{i i}+S_{t}^{i i}+(1-\rho) E_{t-1}^{i j}+S_{t}^{i j} & = \\
(1-\rho) E_{t-1}^{i i}+\Omega_{t}^{i} S_{H, t}^{i}+(1-\rho) E_{t-1}^{i j}+\left(1-\Omega_{t}^{i}\right) S_{H, t}^{i} & =1 .
\end{aligned}
$$

Furthermore, given that $S_{t}^{i i}+S_{t}^{i j}=S_{H, t}^{i}$, with $S_{H, t}^{i}$ being predetermined, choosing $S_{t}^{i i}$ determines $S_{t}^{i j}$ :

$$
S_{t}^{i j}=1-(1-\rho)\left[E_{t-1}^{i i}+E_{t-1}^{i j}\right]-S_{t}^{i i} .
$$

such that (D.7), (D.9), (D.11), and (D.13) become, respectively,

$$
\begin{aligned}
E_{t}^{i j} & =(1-\rho) E_{t-1}^{i j}+\tau_{t}^{j}\left(\theta_{t}^{i j}\right)^{\gamma}\left[1-(1-\rho)\left[E_{t-1}^{i i}+E_{t-1}^{i j}\right]-S_{t}^{i i}\right] \\
E_{t}^{j i} & =(1-\rho) E_{t-1}^{j i}+\tau_{t}^{i}\left(\theta_{t}^{j i}\right)^{\gamma}\left[1-(1-\rho)\left[E_{t-1}^{j j}+E_{t-1}^{j i}\right]-S_{t}^{j j}\right] \\
U_{t}^{j i} & =\left[1-\tau_{t}^{i}\left(\theta_{t}^{j i}\right)^{\gamma}\right]\left[1-(1-\rho)\left[E_{t-1}^{i i}+E_{t-1}^{i j}\right]-S_{t}^{i i}\right] \\
U_{t}^{i j} & =\left[1-\tau_{t}^{j}\left(\theta_{t}^{i j}\right)^{\gamma}\right]\left[1-(1-\rho)\left[E_{t-1}^{j j}+E_{t-1}^{j i}\right]-S_{t}^{j j}\right] .
\end{aligned}
$$


Finally, use constraints (D.10), (D.12), (D.19), and (D.20) to substitute out all four types of unemployment in (D.4) and (D.5), respectively. The cooperative social planner thus maximizes

$$
\begin{aligned}
& \mathbf{E}_{\mathbf{0}} \sum_{t=0}^{\infty} \beta^{t}\left[\mathcal{U}_{t}^{i}\left(C_{t}^{i}, l_{t}^{i}\right)+\mathcal{U}_{t}^{j}\left(C_{t}^{j}, l_{t}^{j}\right)\right]= \\
& \mathbf{E}_{\mathbf{0}} \sum_{t=0}^{\infty}\left[\frac{\left[\left(C_{t}^{i}\right)^{b}\left(l_{t}^{i}\right)^{1-b}\right]^{1-\sigma}-1}{1-\sigma}+\frac{\left[\left(C_{t}^{j}\right)^{b}\left(l_{t}^{j}\right)^{1-b}\right]^{1-\sigma}-1}{1-\sigma}\right]
\end{aligned}
$$

subject to the following constraints:

$$
\begin{aligned}
& C_{t}^{i}=\left\{\left(1-\alpha_{2}\right)^{\frac{1}{\nu_{2}}}\left(C_{H, t}^{i}\right)^{\frac{\nu_{2}-1}{\nu_{2}}}+\left(\alpha_{2}\right)^{\frac{1}{\nu_{2}}}\left(C_{F, t}^{i}\right)^{\frac{\nu_{2}-1}{\nu_{2}}}\right\}^{\frac{\nu_{2}}{\nu_{2}-1}} \\
& C_{t}^{j}=\left\{\left(1-\alpha_{2}\right)^{\frac{1}{\nu_{2}}}\left(C_{H, t}^{j}\right)^{\frac{\nu_{2}-1}{\nu_{2}}}+\left(\alpha_{2}\right)^{\frac{1}{\nu_{2}}}\left(C_{F, t}^{j}\right)^{\frac{\nu_{2}-1}{\nu_{2}}}\right\}^{\frac{\nu_{2}}{\nu_{2}-1}} \\
& l_{t}^{i}=1-\left\{\left(1-\alpha_{1}\right)^{1 / \nu_{1}}\left[E_{t}^{i i}+\zeta\left(1-\tau_{t}^{i}\left(\theta_{t}^{i i}\right)^{\gamma}\right) S_{t}^{i i}\right]^{\frac{\nu_{1}-1}{\nu_{1}}}+\right. \\
& \left.\left(\alpha_{1}\right)^{1 / \nu_{1}}\left[E_{t}^{i j}+\zeta\left(1-\tau_{t}^{j}\left(\theta_{t}^{i j}\right)^{\gamma}\right)\left(1-(1-\rho)\left(E_{t-1}^{i i}+E_{t-1}^{i j}\right)-S_{t}^{i i}\right)\right]^{\frac{\nu_{1}-1}{\nu_{1}}}\right\}^{\frac{\nu_{1}}{\nu_{1}-1}} \\
& l_{t}^{j}=1-\left\{\left(1-\alpha_{1}\right)^{1 / \nu_{1}}\left[E_{t}^{j j}+\zeta\left(1-\tau_{t}^{j}\left(\theta_{t}^{j j}\right)^{\gamma}\right) S_{t}^{j j}\right]^{\frac{\nu_{1}-1}{\nu_{1}}}+\right. \\
& \left.\left(\alpha_{1}\right)^{1 / \nu_{1}}\left[E_{t}^{j i}+\zeta\left(1-\tau_{t}^{i}\left(\theta_{t}^{j i}\right)^{\gamma}\right)\left(1-(1-\rho)\left(E_{t-1}^{j j}+E_{t-1}^{j i}\right)-S_{t}^{j j}\right)\right]^{\frac{\nu_{1}-1}{\nu_{1}}}\right\}^{\frac{\nu_{1}}{\nu_{1}-1}} \\
& E_{t}^{i i}=(1-\rho) E_{t-1}^{i i}+\tau_{t}^{i}\left(\theta_{t}^{i i}\right)^{\gamma} S_{t}^{i i} \\
& E_{t}^{i j}=(1-\rho) E_{t-1}^{i j}+\tau_{t}^{j}\left(\theta_{t}^{i j}\right)^{\gamma}\left[1-(1-\rho)\left(E_{t-1}^{i i}+E_{t-1}^{i j}\right)-S_{t}^{i i}\right] \\
& E_{t}^{j j}=(1-\rho) E_{t-1}^{j j}+\tau_{t}^{j}\left(\theta_{t}^{j j}\right)^{\gamma} S_{t}^{j j} \\
& E_{t}^{j i}=(1-\rho) E_{t-1}^{j i}+\tau_{t}^{i}\left(\theta_{t}^{j i}\right)^{\gamma}\left[1-(1-\rho)\left(E_{t-1}^{j j}+E_{t-1}^{j i}\right)-S_{t}^{j j}\right] \\
& {\left[A_{t}^{i}\left(E_{t}^{i i}+E_{t}^{j i}\right)\right]^{1-\alpha}=C_{H, t}^{i}+C_{F, t}^{j}+\kappa \theta_{t}^{i i} S_{t}^{i i}+\kappa \theta_{t}^{j i}\left[1-(1-\rho)\left(E_{t-1}^{j j}+E_{t-1}^{j i}\right)-S_{t}^{j j}\right]} \\
& {\left[A_{t}^{j}\left(E_{t}^{j j}+E_{t}^{i j}\right)\right]^{1-\alpha}=C_{H, t}^{j}+C_{F, t}^{i}+\kappa \theta_{t}^{j j} S_{t}^{j j}+\kappa \theta_{t}^{i j}\left[1-(1-\rho)\left(E_{t-1}^{i i}+E_{t-1}^{i j}\right)-S_{t}^{i i}\right] .}
\end{aligned}
$$

Defining the Lagrange multiplier on the two resource constraints as $\lambda_{t, R C}^{i}$ and $\lambda_{t, R C}^{j}$, respectively, and the Lagrange multipliers for the law of motion of each of the four types of employment as $\lambda_{E, t}^{i i}, \lambda_{E, t}^{i j}, \lambda_{E, t}^{j j}$, and $\lambda_{E, t}^{i j}$, the first-order conditions with respect 
to domestic and imported consumption state as follows:

$$
\begin{aligned}
& {\left[C_{H, t}^{i} ; C_{F, t}^{j}\right]: \lambda_{R C, t}^{i}=U_{c, t}^{i}\left[\frac{\left(1-\alpha_{2}\right) C_{t}^{i}}{C_{H, t}^{i}}\right]^{\frac{1}{\nu_{2}}}=U_{c, t}^{j}\left[\frac{\alpha_{2} C_{t}^{j}}{C_{F, t}^{j}}\right]^{\frac{1}{\nu_{2}}}} \\
& {\left[C_{F, t}^{i} ; C_{H, t}^{j}\right]: \lambda_{R C, t}^{j}=U_{c, t}^{i}\left[\frac{\alpha_{2} C_{t}^{i}}{C_{F, t}^{i}}\right]^{\frac{1}{\nu_{2}}}=U_{c, t}^{j}\left[\frac{\left(1-\alpha_{2}\right) C_{t}^{j}}{C_{H, t}^{j}}\right]^{\frac{1}{\nu_{2}}}} \\
& {\left[\theta_{t}^{i i}\right]: \frac{U_{l, t}^{i}}{U_{c, t}^{i}}\left[\frac{\left(1-\alpha_{1}\right)\left(1-l_{t}^{i}\right)}{E_{t}^{i i}+\zeta U_{t}^{i i}}\right]^{\frac{1}{\nu_{1}}} \zeta \gamma \tau_{t}^{i}\left(\theta_{t}^{i i}\right)^{\gamma-1}=\frac{\lambda_{E, t}^{i i}}{U_{c, t}^{i}} \gamma \tau_{t}^{i}\left(\theta_{t}^{i i}\right)^{\gamma-1}+\kappa \frac{\lambda_{R C, t}^{i}}{U_{c, t}^{i}}(\mathrm{D}} \\
& {\left[\theta_{t}^{i j}\right]: \frac{U_{l, t}^{i}}{U_{c, t}^{i}}\left[\frac{\alpha_{1}\left(1-l_{t}^{i}\right)}{E_{t}^{i j}+\zeta U_{t}^{i j}}\right]^{\frac{1}{\nu_{1}}} \zeta \gamma \tau_{t}^{j}\left(\theta_{t}^{i j}\right)^{\gamma-1}=\frac{\lambda_{E, t}^{i j}}{U_{c, t}^{i}} \gamma \tau_{t}^{j}\left(\theta_{t}^{i j}\right)^{\gamma-1}+\kappa \frac{\lambda_{R C, t}^{j}}{U_{c, t}^{i}}} \\
& {\left[E_{t}^{i i}\right]: \frac{U_{l, t}^{i}}{U_{c, t}^{i}}\left[\frac{\left(1-\alpha_{1}\right)\left(1-l_{t}^{i}\right)}{E_{t}^{i i}+\zeta U_{t}^{i i}}\right]^{\frac{1}{\nu_{1}}}=\frac{\lambda_{E, t}^{i i}}{U_{c, t}^{i}}+\frac{\lambda_{R C, t}^{i}}{U_{c, t}^{i}} \frac{(1-\alpha) Y_{t}^{i}}{E_{t}^{i}}+\beta(1-\rho) \frac{U_{c, t+1}^{i}}{U_{c, t}^{i}} \ldots} \\
& \ldots\left\{\frac{U_{l, t+1}^{i}}{U_{c, t+1}^{i}}\left[\frac{\alpha_{1}\left(1-l_{t+1}^{i}\right)}{E_{t+1}^{i j}+\zeta U_{t+1}^{i j}}\right]^{\frac{1}{\nu_{1}}} \zeta\left(1-\tau_{t+1}^{j}\left(\theta_{t+1}^{i j}\right)^{\gamma}\right)-\frac{\lambda_{E, t+1}^{i i}}{U_{c, t+1}^{i}}+\frac{\lambda_{E, t+1}^{i j}}{U_{c, t+1}^{i}} \tau_{t+1}^{j}\left(\theta_{t+1}^{i j}\right)^{\gamma}+\frac{\lambda_{R C, t+1}^{j}}{U_{c, t+1}^{i}} \kappa \theta_{t+1}^{i j}\right\} \\
& {\left[E_{t}^{i j}\right]: \frac{U_{l, t}^{i}}{U_{c, t}^{i}}\left[\frac{\alpha_{1}\left(1-l_{t}^{i}\right)}{E_{t}^{i j}+\zeta U_{t}^{i j}}\right]^{\frac{1}{\nu_{1}}}=\frac{\lambda_{E, t}^{i j}}{U_{c, t}^{i}}+\frac{\lambda_{R C, t}^{j}}{U_{c, t}^{i}} \frac{(1-\alpha) Y_{t}^{j}}{E_{t}^{j}}+\beta(1-\rho) \frac{U_{c, t+1}^{i}}{U_{c, t}^{i}} \ldots} \\
& \ldots\left\{\frac{U_{l, t+1}^{i}}{U_{c, t+1}^{i}}\left[\frac{\alpha_{1}\left(1-l_{t+1}^{i}\right)}{E_{t+1}^{i j}+\zeta U_{t+1}^{i j}}\right]^{\frac{1}{\nu_{1}}} \zeta\left(1-\tau_{t+1}^{j}\left(\theta_{t+1}^{i j}\right)^{\gamma}\right)-\frac{\lambda_{E, t+1}^{i j}}{U_{c, t+1}^{i}}\left(1-\tau_{t+1}^{j}\left(\theta_{t+1}^{i j}\right)^{\gamma}\right)+\frac{\lambda_{R C, t+1}^{j}}{U_{c, t+1}^{i}} \kappa \theta_{t+1}^{i j}\right\} \\
& {\left[S_{t}^{i i}\right]: \zeta \frac{U_{l, t}^{i}}{U_{c, t}^{i}}\left\{\left[\frac{\alpha_{1}\left(1-l_{t}^{i}\right)}{E_{t}^{i j}+\zeta U_{t}^{i j}}\right]^{\frac{1}{\nu_{1}}}\left(1-\tau_{t}^{j}\left(\theta_{t}^{i j}\right)^{\gamma}\right)-\left[\frac{\left(1-\alpha_{1}\right)\left(1-l_{t}^{i}\right)}{E_{t}^{i i}+\zeta U_{t}^{i i}}\right]^{\frac{1}{\nu_{1}}}\left(1-\tau_{t}^{i}\left(\theta_{t}^{i i}\right)^{\gamma}\right)\right\}=\ldots} \\
& \frac{\lambda_{E, t}^{i i}}{U_{c, t}^{i}} \tau_{t}^{i}\left(\theta_{t}^{i i}\right)^{\gamma}-\frac{\lambda_{E, t}^{i j}}{U_{c, t}^{i}} \tau_{t}^{j}\left(\theta_{t}^{i j}\right)^{\gamma}+\kappa \theta_{t}^{i i} \frac{\lambda_{R C, t}^{i}}{U_{c, t}^{i}}-\kappa \theta_{t}^{i j} \frac{\lambda_{R C, t}^{j}}{U_{c, t}^{i}} .
\end{aligned}
$$

Further combining (D.23) and (D.24) with (D.21) yields, respectively,

$$
\begin{aligned}
& \frac{\lambda_{E, t}^{i i}}{U_{c, t}^{i}}=\zeta \frac{U_{l, t}^{i}}{U_{c, t}^{i}}\left[\frac{\left(1-\alpha_{1}\right)\left(1-l_{t}^{i}\right)}{E_{t}^{i i}+\zeta U_{t}^{i i}}\right]^{\frac{1}{\nu_{1}}}-\frac{\kappa}{q_{t}^{i i} \gamma}\left[\frac{\left(1-\alpha_{2}\right) C_{t}^{i}}{C_{H, t}^{i}}\right]^{\frac{1}{\nu_{2}}} \\
& \frac{\lambda_{E, t}^{i j}}{U_{c, t}^{i}}=\zeta \frac{U_{l, t}^{i}}{U_{c, t}^{i}}\left[\frac{\alpha_{1}\left(1-l_{t}^{i}\right)}{E_{t}^{i j}+\zeta U_{t}^{i j}}\right]^{\frac{1}{\nu_{1}}}-\frac{\kappa}{q_{t}^{i j} \gamma}\left[\frac{\alpha_{2} C_{t}^{i}}{C_{F, t}^{i}}\right]^{\frac{1}{\nu_{2}}} .
\end{aligned}
$$


Combining (D.25) with (D.21), (D.28), and (D.29) results in

$$
\begin{gathered}
\frac{U_{l, t}^{i}}{U_{c, t}^{i}}(1-\zeta)\left[\frac{\left(1-\alpha_{1}\right)\left(1-l_{t}^{i}\right)}{E_{t}^{i i}+\zeta U_{t}^{i i}}\right]^{\frac{1}{\nu_{1}}}=\left[\frac{\left(1-\alpha_{2}\right) C_{t}^{i}}{C_{H, t}^{i}}\right]^{\frac{1}{\nu_{2}}}\left[\frac{(1-\alpha) Y_{t}^{i}}{E_{t}^{i}}-\frac{\kappa}{q_{t}^{i i} \gamma}\right]+\beta(1-\rho) \frac{U_{c, t+1}^{i}}{U_{c, t}^{i}} \ldots \\
\cdots\left\{\frac{U_{l, t+1}^{i}}{U_{c, t+1}^{i}} \zeta\left[\left[\frac{\alpha_{1}\left(1-l_{t+1}^{i}\right)}{E_{t+1}^{i j}+\zeta U_{t+1}^{i j}}\right]^{\frac{1}{\nu_{1}}}-\left[\frac{\left(1-\alpha_{1}\right)\left(1-l_{t+1}^{i}\right)}{E_{t+1}^{i i}+\zeta U_{t+1}^{i i}}\right]^{\frac{1}{\nu_{1}}}\right]+\frac{\kappa}{q_{t+1}^{i i} \gamma}\left[\frac{\left(1-\alpha_{2}\right) C_{t+1}^{i}}{C_{H, t+1}^{i}}\right]^{\frac{1}{\nu_{2}}}+\left[\frac{\alpha_{2} C_{t+1}^{i}}{C_{H, t+1}^{i}}\right]^{\frac{1}{\nu_{2}}} \kappa \frac{\gamma-1}{\gamma} \theta_{t+1}^{i j}\right\} .
\end{gathered}
$$

Rewriting the efficiency condition with respect to $S_{t}^{i i},(\mathrm{D} .26)$, using (D.21), (D.22), (D.28), and (D.29) yields

$$
\begin{gathered}
\frac{U_{l, t}^{i}}{U_{c, t}^{i}} \zeta\left\{\left[\frac{\alpha_{1}\left(1-l_{t}^{i}\right)}{E_{t}^{i j}+\zeta U_{t}^{i j}}\right]^{\frac{1}{\nu_{1}}}-\left[\frac{\left(1-\alpha_{1}\right)\left(1-l_{t}^{i}\right)}{E_{t}^{i i}+\zeta U_{t}^{i i}}\right]^{\frac{1}{\nu_{1}}}\right\}= \\
\frac{1-\gamma}{\gamma} \kappa\left\{\left[\frac{\alpha_{2} C_{t}^{i}}{C_{F, t}^{i}}\right]^{\frac{1}{\nu_{2}}} \theta_{t}^{i j}-\left[\frac{\left(1-\alpha_{2}\right) C_{t}^{i}}{C_{H, t}^{i}}\right]^{\frac{1}{\nu_{2}}} \theta_{t}^{i i}\right\} .
\end{gathered}
$$

Combining the efficient migration decision, (D.31), with (D.30) leads to the efficiency condition for native workers

$$
\begin{aligned}
& \frac{U_{l, t}^{i}}{U_{c, t}^{i}}(1-\zeta)\left[\frac{\left(1-\alpha_{1}\right)\left(1-l_{t}^{i}\right)}{E_{t}^{i i}+\zeta U_{t}^{i i}}\right]^{\frac{1}{\nu_{1}}}=\left[\frac{\left(1-\alpha_{2}\right) C_{t}^{i}}{C_{H, t}^{i}}\right]^{\frac{1}{\nu_{2}}}\left[\frac{(1-\alpha) Y_{t}^{i}}{E_{t}^{i}}-\frac{\kappa}{q_{t}^{i i} \gamma}\right](\mathrm{D} \\
& +\beta(1-\rho) \frac{U_{c, t+1}^{i}}{U_{c, t}^{i}}\left[\frac{\left(1-\alpha_{2}\right) C_{t+1}^{i}}{C_{H, t+1}^{i}}\right]^{\frac{1}{\nu_{2}}} \frac{\kappa}{q_{t+1}^{i i}} \frac{1-(1-\gamma) f_{t+1}^{i i}}{\gamma}
\end{aligned}
$$

where

$$
\begin{gathered}
\beta(1-\rho) \frac{U_{c, t+1}^{i}}{U_{c, t}^{i}}\left[\frac{\left(1-\alpha_{2}\right) C_{t+1}^{i}}{C_{H, t+1}^{i}}\right]^{\frac{1}{\nu_{2}}} \frac{\kappa}{q_{t+1}^{i i}} \frac{1-(1-\gamma) f_{t+1}^{i i}}{\gamma}= \\
\underbrace{(1-\rho) \mathbf{E}_{\mathbf{t}}\left\{\mathcal{Q}_{t, t+1} V_{J, t+1}^{i i}\right\}}_{\text {continuation value for firms }}+\underbrace{(1-\rho) \mathbf{E}_{\mathbf{t}} \mathcal{Q}_{t, t+1}\left(1-f_{t+1}^{i i}\right) V_{W, t+1}^{i i}}_{\text {continuation value for workers }} .
\end{gathered}
$$

Equivalently, for migrant workers combining (D.26) with (D.22), (D.28), and (D.29) results in

$$
\begin{aligned}
& \frac{U_{l, t}^{i}}{U_{c, t}^{i}}(1-\zeta)\left[\frac{\alpha_{1}\left(1-l_{t}^{i}\right)}{E_{t}^{i j}+\zeta U_{t}^{i j}}\right]^{\frac{1}{\nu_{1}}}= \\
& {\left[\frac{\alpha_{2} C_{t}^{i}}{C_{F, t}^{i}}\right]^{\frac{1}{\nu_{2}}}\left[\frac{(1-\alpha) Y_{t}^{j}}{E_{t}^{j}}-\frac{\kappa}{q_{t}^{i j} \gamma}\right]+\beta(1-\rho) \frac{U_{c, t+1}^{i}}{U_{c, t}^{i}}\left[\frac{\alpha_{2} C_{t+1}^{i}}{C_{F, t+1}^{i}}\right]^{\frac{1}{\nu_{2}}} \frac{\kappa}{q_{t+1}^{i j}} \frac{1-(1-\gamma) f_{t+1}^{i j}}{\gamma} .}
\end{aligned}
$$

Finally, combining the first-order condition with respect to $S_{t}^{i i}$ with equations (D.28) and (D.29) yields the following efficient migration decision,

$$
\frac{U_{l, t}^{i}}{U_{c, t}^{i}} \zeta\left\{\left[\frac{\left(1-\alpha_{1}\right)\left(1-l_{t}^{i}\right)}{E_{t}^{i i}+\zeta U_{t}^{i i}}\right]^{\frac{1}{\nu_{1}}}-\left[\frac{\alpha_{1}\left(1-l_{t}^{i}\right)}{E_{t}^{i j}+\zeta U_{t}^{i j}}\right]^{\frac{1}{\nu_{1}}}\right\}=\frac{1-\gamma}{\gamma}\left\{f_{t}^{i i} V_{J, t}^{i i}-f_{t}^{i j} V_{J, t}^{i j}\right\} .
$$




\section{Appendix E. Efficient steady state}

At the symmetric steady state, we assume that $A^{i}=A^{j}=Z^{i}=Z^{j}=1$. The firstorder efficiency conditions with respect to labor market tightness and the four types of employment simplify to

$$
\begin{aligned}
\frac{1-b}{b} \frac{C}{l} \zeta & =\frac{1-\gamma}{\gamma} \kappa \theta \\
\frac{1-\alpha}{E^{\alpha}}-\frac{\kappa}{q}[1-\beta(1-\rho)] & =\frac{1-b}{b} \frac{C}{l}\left[1+\zeta \frac{1-f}{f}(1-\beta(1-\rho))\right] .
\end{aligned}
$$

\section{Appendix F. A purely quadratic welfare criterion-Benchmark model with- out labor mobility}

We approximate households' utility function to the second order. The linear terms in consumption and leisure are then substituted out by the feasibility constraints faced by the policymaker, also approximated to the second order. The resulting second-order expression contains linear and quadratic terms. We show that all linear terms can be substituted out with linear combinations of second-order terms, such that our welfare criterion is purely quadratic.

Appendix F.1. Taylor expansion of the utility function and feasibility constraints

Define $\hat{x}_{t}^{i} \equiv \log \left(X_{t}^{i}\right)-\log (X)$ as the $\log$ deviation from the symmetric steady state for a generic variable $X_{t}^{i}$ specific to region $i$. A second-order log-linear approximation of the instantaneous utility function in region $i$ yields

$$
U_{t}^{i} \simeq U^{i}+U_{c}^{i} \hat{c}_{t}^{i}+U_{l}^{i} \hat{l}_{t}^{i}+\frac{1}{2} U_{c c}^{i}\left(\hat{c}_{t}^{i}\right)^{2}+\frac{1}{2} U_{l l}^{i}\left(\hat{l}_{t}^{i}\right)^{2}+U_{c l}^{i} \hat{c}_{t}^{i} \hat{l}_{t}^{i}+\text { t.i.p. }
$$

where $U_{x}^{i}$ denotes the first-order derivative of the utility function with respect to $\log X_{t}^{i}$, and where t.i.p. stands for terms that are independent of policy. For $\sigma=1$, the above expression simplifies to

$$
\frac{U_{t}^{i}-U^{i}}{U_{c}^{i}} \simeq \hat{c}_{t}^{i}+\frac{1-b}{b} \hat{l}_{t}^{i}+t . i . p
$$

The consumption aggregator for region $i$, up to second order, reads as

$\hat{c}_{t}^{i}=-\frac{1}{2} \frac{\nu_{2}-1}{\nu_{2}}\left(\hat{c}_{t}^{i}\right)^{2}+\left(1-\alpha_{2}\right)\left[\hat{c}_{H, t}^{i}+\frac{1}{2} \frac{\nu_{2}-1}{\nu_{2}}\left(\hat{c}_{H, t}^{i}\right)^{2}\right]+\alpha_{2}\left[\hat{c}_{F, t}^{i}+\frac{1}{2} \frac{\nu_{2}-1}{\nu_{2}}\left(\hat{c}_{F, t}^{i}\right)^{2}\right]$,

while the first feasibility constraint for region $i$,

$$
\left(\frac{A_{t}^{i} E_{t}^{i}}{\Delta_{t}^{i}}\right)^{(1-\alpha)}=C_{H, t}^{i}+C_{F, t}^{j}+\frac{\kappa}{\omega}\left(\theta_{t}^{i}\right)^{1-\gamma}\left[E_{t}^{i}-\left(1-\rho^{i}\right) E_{t-1}^{i}\right]
$$


up to the second order states as

$$
\begin{aligned}
& (1-\alpha)\left(E^{i}\right)^{1-\alpha}\left[-\hat{\Delta}_{t}^{i}+\hat{e}_{t}^{i}+\frac{1}{2}(1-\alpha)\left(\hat{e}_{t}^{i}\right)^{2}+(1-\alpha) \hat{a}_{t}^{i} \hat{e}_{t}^{i}\right]= \\
& C_{H}\left[\hat{c}_{H, t}^{i}+\frac{1}{2}\left(\hat{c}_{H, t}^{i}\right)^{2}\right]+C_{F}\left[\hat{c}_{F, t}^{j}+\frac{1}{2}\left(\hat{c}_{F, t}^{j}\right)^{2}\right]+ \\
& \frac{\kappa}{q} E\left[(1-\gamma) \hat{\theta}_{t}^{i}+\hat{e}_{t}^{i}+\frac{1}{2}(1-\gamma)^{2}\left(\hat{\theta}_{t}^{i}\right)^{2}+\frac{1}{2}\left(\hat{e}_{t}^{i}\right)^{2}+(1-\gamma) \hat{\theta}_{t}^{i} \hat{e}_{t}^{i}\right]- \\
& \frac{\kappa}{q} E(1-\rho)\left[(1-\gamma) \hat{\theta}_{t}^{i}+\hat{e}_{t-1}^{i}+\frac{1}{2}(1-\gamma)^{2}\left(\hat{\theta}_{t}^{i}\right)^{2}+\frac{1}{2}\left(\hat{e}_{t-1}^{i}\right)^{2}+(1-\gamma) \hat{\theta}_{t}^{i} \hat{e}_{t-1}^{i}\right],
\end{aligned}
$$

which can be rewritten as follows:

$$
\begin{aligned}
& \hat{c}_{H, t}^{i}=\frac{(1-\alpha) E^{1-\alpha}}{C_{H}}\left[-\hat{\Delta}_{t}^{i}+\hat{e}_{t}^{i}+\frac{1}{2}(1-\alpha)\left(\hat{e}_{t}^{i}\right)^{2}+(1-\alpha) \hat{a}_{t}^{i} \hat{e}_{t}^{i}\right]- \\
& \frac{\kappa}{q} \frac{E}{C_{H}}\left[(1-\gamma) \hat{\theta}_{t}^{i}+\hat{e}_{t}^{i}+\frac{1}{2}(1-\gamma)^{2}\left(\hat{\theta}_{t}^{i}\right)^{2}+\frac{1}{2}\left(\hat{e}_{t}^{i}\right)^{2}+(1-\gamma) \hat{\theta}_{t}^{i} \hat{e}_{t}^{i}\right]+ \\
& \frac{\kappa}{q} \frac{E}{C_{H}}(1-\rho)\left[(1-\gamma) \hat{\theta}_{t}^{i}+\hat{e}_{t-1}^{i}+\frac{1}{2}(1-\gamma)^{2}\left(\hat{\theta}_{t}^{i}\right)^{2}+\frac{1}{2}\left(\hat{e}_{t-1}^{i}\right)^{2}+(1-\gamma) \hat{\theta}_{t}^{i} \hat{e}_{t-1}^{i}\right]- \\
& \frac{1}{2}\left(\hat{c}_{H, t}^{i}\right)^{2}-\frac{\alpha_{2}}{1-\alpha_{2}}\left[\hat{c}_{F, t}^{j}+\frac{1}{2}\left(\hat{c}_{F, t}^{j}\right)^{2}\right] .
\end{aligned}
$$

Combining (F.3) and (F.6) yields

$$
\begin{aligned}
& \hat{c}_{t}^{i}=-\frac{1}{2} \frac{\nu_{2}-1}{\nu_{2}}\left(\hat{c}_{t}^{i}\right)^{2}+\frac{1}{2} \frac{\nu_{2}-1}{\nu_{2}}\left(1-\alpha_{2}\right)\left(\hat{c}_{H, t}^{i}\right)^{2}+\alpha_{2}\left[\hat{c}_{F, t}^{i}+\frac{1}{2} \frac{\nu_{2}-1}{\nu_{2}}\left(\hat{c}_{F, t}^{i}\right)^{2}\right]+ \\
& \frac{(1-\alpha) E^{1-\alpha}}{C}\left[-\hat{\Delta}_{t}^{i}+\hat{e}_{t}^{i}+\frac{1}{2}(1-\alpha)\left(\hat{e}_{t}^{i}\right)^{2}+(1-\alpha) \hat{a}_{t}^{i} \hat{e}_{t}^{i}\right]- \\
& \frac{\kappa}{q} \frac{E}{C}\left[(1-\gamma) \hat{\theta}_{t}^{i}+\hat{e}_{t}^{i}+\frac{1}{2}(1-\gamma)^{2}\left(\hat{\theta}_{t}^{i}\right)^{2}+\frac{1}{2}\left(\hat{e}_{t}^{i}\right)^{2}+(1-\gamma) \hat{\theta}_{t}^{i} \hat{e}_{t}^{i}\right]+ \\
& \frac{\kappa}{q} \frac{E}{C}(1-\rho)\left[(1-\gamma) \hat{\theta}_{t}^{i}+\hat{e}_{t-1}^{i}+\frac{1}{2}(1-\gamma)^{2}\left(\hat{\theta}_{t}^{i}\right)^{2}+\frac{1}{2}\left(\hat{e}_{t-1}^{i}\right)^{2}+(1-\gamma) \hat{\theta}_{t}^{i} \hat{e}_{t-1}^{i}\right]- \\
& \frac{1}{2}\left(1-\alpha_{2}\right)\left(\hat{c}_{H, t}^{i}\right)^{2}-\alpha_{2}\left[\hat{c}_{F, t}^{j}+\frac{1}{2}\left(\hat{c}_{F, t}^{j}\right)^{2}\right] .
\end{aligned}
$$

An equivalent expression for total consumption in region $j, \hat{c}_{t}^{j}$, can be derived by combining region $j$ 's consumption aggregator with its feasibility constraint. The second feasibility constraint for region $i$ relates to households' time allocation. Up to second 
order, this constraint states as follows:

$$
\begin{aligned}
\hat{l}_{t}^{i}= & -\frac{1}{2}\left(\hat{l}_{t}^{i}\right)^{2}-\frac{E}{l}\left(1+\zeta \frac{1-f}{f}\right) \hat{e}_{t}^{i}+\frac{E}{l} \frac{\rho \zeta \gamma}{f} \hat{\theta}_{t}^{i}+\frac{E}{l}(1-\rho) \zeta \frac{1-f}{f} \hat{e}_{t-1}^{i} \\
& -\frac{1}{2} \frac{E}{l}\left(1+\zeta \frac{1-f}{f}\right)\left(\hat{e}_{t}^{i}\right)^{2}-\frac{1}{2} \frac{E}{l} \frac{\rho \gamma^{2} \zeta}{f}\left(\hat{\theta}_{t}^{i}\right)^{2}+\frac{1}{2} \frac{E}{l}(1-\rho) \zeta \frac{1-f}{f}\left(\hat{e}_{t-1}^{i}\right)^{2} \\
& +\frac{\zeta \gamma}{f} \frac{E}{l} \hat{\theta}_{t}^{i}\left[\hat{e}_{t}^{i}-(1-\rho) \hat{e}_{t-1}^{i}\right]+t . i . p .
\end{aligned}
$$

A second-order approximation (around a symmetric steady state) of the instantaneous utility functions of the two regions constituting a monetary union therefore reads as follows:

$$
\left[\hat{c}_{t}^{i}+\frac{1-b}{b} \hat{l}_{t}^{i}\right]+\left[\hat{c}_{t}^{j}+\frac{1-b}{b} \hat{l}_{t}^{j}\right]+\text { t.i.p. },
$$

where $\hat{c}_{t}^{i}$ and $\hat{l}_{t}^{i}$ are given by equations (F.7) and (F.8), respectively. Equivalent equations can be derived for consumption and leisure in region $j$.

\section{Appendix F.2. Linear terms}

One can then collect all linear terms appearing in the generic period $t$ so that all first-order terms in the approximated discounted and weighted lifetime utility of the representative household in both regions read as

$$
\begin{array}{r}
\sum_{t=0}^{\infty} \beta^{t}\left[\Lambda_{1}\left(\hat{e}_{t}^{i}+\hat{e}_{t}^{j}\right)+\Lambda_{2}\left(\hat{\theta}_{t}^{i}+\hat{\theta}_{t}^{j}\right)+\Lambda_{3}\left(\hat{e}_{t-1}^{i}+\hat{e}_{t-1}^{j}\right)+\Lambda_{4}\left(\hat{c}_{F, t}^{i}+\hat{c}_{F, t}^{j}\right)\right]= \\
\Lambda_{3}\left(\hat{e}_{-1}^{i}+\hat{e}_{-1}^{j}\right)+\sum_{t=0}^{\infty} \beta^{t}\left[\left(\Lambda_{1}+\beta \Lambda_{3}\right)\left(\hat{e}_{t}^{i}+\hat{e}_{t}^{j}\right)+\Lambda_{2}\left(\hat{\theta}_{t}^{i}+\hat{\theta}_{t}^{j}\right)+\Lambda_{4}\left(\hat{c}_{F, t}^{i}+\hat{c}_{F, t}^{j}\right)\right],
\end{array}
$$

where the term $\Lambda_{3}\left(\hat{e}_{-1}^{i}+\hat{e}_{-1}^{j}\right)$ is given at time $t=0$ and is thus independent of policy. By steady-state efficiency, the coefficients on employment, $\Lambda_{1}+\beta \Lambda_{3}$, on labor market tightness, $\Lambda_{2}$, and on imported consumption, $\Lambda_{4}$, are all equal to zero. Hence, all linear terms cancel out and we are left with quadratic terms only. 
Appendix F.3. Quadratic terms for endogenous variables

Leaving the cross-products with the shocks for the next section, we collect the purely quadratic terms

$$
\begin{aligned}
& \frac{1}{2} \sum_{t=0}^{\infty} \beta^{t}\left\{\Gamma_{1}\left[\left(\hat{e}_{t}^{i}\right)^{2}+\left(\hat{e}_{t}^{j}\right)^{2}\right]+\Gamma_{2}\left[\left(\hat{e}_{t-1}^{i}\right)^{2}+\left(\hat{e}_{t-1}^{j}\right)^{2}\right]+\Gamma_{\theta}\left[\left(\hat{\theta}_{t}^{i}\right)^{2}+\left(\hat{\theta}_{t}^{j}\right)^{2}\right]+\right. \\
& \Gamma_{c}\left[\left(\hat{c}_{t}^{i}\right)^{2}+\left(\hat{c}_{t}^{j}\right)^{2}\right]+\Gamma_{c_{H}}\left[\left(\hat{c}_{H, t}^{i}\right)^{2}+\left(\hat{c}_{H, t}^{j}\right)^{2}\right]+\Gamma_{c_{F}}\left[\left(\hat{c}_{F, t}^{i}\right)^{2}+\left(\hat{c}_{F, t}^{j}\right)^{2}\right]+ \\
& \left.\Gamma_{l}\left[\left(\hat{l}_{t}^{i}\right)^{2}+\left(\hat{l}_{t}^{j}\right)^{2}\right]+\Gamma_{\Delta}\left[\hat{\Delta}_{t}^{i}+\hat{\Delta}_{t}^{j}\right]+2 \Gamma_{3} \hat{\theta}_{t}^{i}\left[\hat{e}_{t}^{i}-(1-\rho) \hat{e}_{t-1}^{i}\right]+2 \Gamma_{3} \hat{\theta}_{t}^{j}\left[\hat{e}_{t}^{j}-(1-\rho) \hat{e}_{t-1}^{j}\right]\right\} \\
& =\frac{1}{2}[\underbrace{\left(\hat{e}_{-1}^{i}\right)^{2}+\left(\hat{e}_{-1}^{j}\right)^{2}}_{\text {t.i.p. }}]+\frac{1}{2} \sum_{t=0}^{\infty} \beta^{t}\left\{\left(\Gamma_{1}+\beta \Gamma_{2}\right)\left[\left(\hat{e}_{t}^{i}\right)^{2}+\left(\hat{e}_{t}^{j}\right)^{2}\right]+\Gamma_{\theta}\left[\left(\hat{\theta}_{t}^{i}\right)^{2}+\left(\hat{\theta}_{t}^{j}\right)^{2}\right]+\right. \\
& \Gamma_{c}\left[\left(\hat{c}_{t}^{i}\right)^{2}+\left(\hat{c}_{t}^{j}\right)^{2}\right]+\Gamma_{c_{H}}\left[\left(\hat{c}_{H, t}^{i}\right)^{2}+\left(\hat{c}_{H, t}^{j}\right)^{2}\right]+\Gamma_{c_{F}}\left[\left(\hat{c}_{F, t}^{i}\right)^{2}+\left(\hat{c}_{F, t}^{j}\right)^{2}\right]+ \\
& \left.\Gamma_{l}\left[\left(\hat{l}_{t}^{i}\right)^{2}+\left(\hat{l}_{t}^{j}\right)^{2}\right]+\Gamma_{\Delta}\left[\hat{\Delta}_{t}^{i}+\hat{\Delta}_{t}^{j}\right]+2 \Gamma_{3} \hat{\theta}_{t}^{i}\left[\hat{e}_{t}^{i}-(1-\rho) \hat{e}_{t-1}^{i}\right]+2 \Gamma_{3} \hat{\theta}_{t}^{j}\left[\hat{e}_{t}^{j}-(1-\rho) \hat{e}_{t-1}^{j}\right]\right\},
\end{aligned}
$$

where, at the symmetric steady state,

$$
\begin{aligned}
\Gamma_{1} & \equiv \frac{E}{C}\left[\frac{(1-\alpha)^{2}}{E^{\alpha}}-\frac{\kappa}{q}-\frac{1-b}{b} \frac{C}{l}\left(1+\zeta \frac{1-f}{f}\right)\right] \\
\Gamma_{2} & \equiv \frac{E}{C}(1-\rho)\left[\frac{\kappa}{q}+\frac{1-b}{b} \frac{C}{l} \zeta \frac{1-f}{f}\right] \\
\Gamma_{\theta}^{i} & \equiv-\frac{E}{C} \frac{\kappa}{q}(1-\gamma) \rho \\
\Gamma_{c} & \equiv-\left(\frac{\nu_{2}-1}{\nu_{2}}\right) ; \Gamma_{c_{H}} \equiv-\frac{1-\alpha_{2}}{\nu_{2}} ; \Gamma_{c_{F}} \equiv-\frac{\alpha_{2}}{\nu_{2}} \\
\Gamma_{l} & \equiv-\frac{1-b}{b} ; \Gamma_{\Delta} \equiv-2 \frac{1-\alpha}{E^{\alpha}} \frac{E}{C} \\
\Gamma_{3} & \equiv \frac{E}{C} \frac{\gamma}{f}\left[\frac{1-b}{b} \frac{C}{l} \zeta-\frac{1-\gamma}{\gamma} \kappa \theta\right]=0 \\
\Gamma_{e} & \equiv \Gamma_{1}+\beta \Gamma_{2}=-\frac{E}{C} \frac{\alpha(1-\alpha)}{E^{\alpha}} .
\end{aligned}
$$

Therefore, the squares appearing in the welfare function are

$$
\begin{aligned}
& \frac{1}{2} \sum_{t=0}^{\infty} \beta^{t}\left\{\Gamma_{e}\left[\left(\hat{e}_{t}^{i}\right)^{2}+\left(\hat{e}_{t}^{j}\right)^{2}\right]+\Gamma_{\theta}\left[\left(\hat{\theta}_{t}^{i}\right)^{2}+\left(\hat{\theta}_{t}^{j}\right)^{2}\right]+\right. \\
& \Gamma_{c}\left[\left(\hat{c}_{t}^{i}\right)^{2}+\left(\hat{c}_{t}^{j}\right)^{2}\right]+\Gamma_{c_{H}}\left[\left(\hat{c}_{H, t}^{i}\right)^{2}+\left(\hat{c}_{H, t}^{j}\right)^{2}\right]+\Gamma_{c_{F}}\left[\left(\hat{c}_{F, t}^{i}\right)^{2}+\left(\hat{c}_{F, t}^{j}\right)^{2}\right]+ \\
& \left.\Gamma_{l}\left[\left(\hat{l}_{t}^{i}\right)^{2}+\left(\hat{l}_{t}^{j}\right)^{2}\right]+\Gamma_{\Delta}\left[\hat{\Delta}_{t}^{i}+\hat{\Delta}_{t}^{j}\right]\right\}+ \text { t.i.p. }
\end{aligned}
$$


Appendix F.4. Cross-products with the shocks

Finally, we are left with the second-order terms where the shocks are multiplied by endogenous variables:

$$
\sum_{t=0}^{\infty} \beta^{t} \Gamma_{a e}\left[\hat{a}_{t}^{i} \hat{e}_{t}^{i}+\hat{a}_{t}^{j} \hat{e}_{t}^{j}\right]+\text { t.i.p. },
$$

where, at the symmetric steady state,

$$
\Gamma_{a e}=\frac{(1-\alpha)^{2}}{E^{\alpha}} \frac{E}{C} .
$$

Appendix F.5. Second-order approximation of the utility function

Collecting all quadratic terms yields

$$
\begin{aligned}
W \simeq & \mathbf{E}_{0} \sum_{t=0}^{\infty} \beta^{t}\left[\left(\frac{U_{t}^{i}-U^{i}}{U_{c}^{i}}\right)+\left(\frac{U_{t}^{j}-U^{j}}{U_{c}^{j}}\right)\right] \\
& \frac{1}{2} \sum_{t=0}^{\infty} \beta^{t}\left\{\Gamma_{e}\left[\left(\hat{e}_{t}^{i}\right)^{2}+\left(\hat{e}_{t}^{j}\right)^{2}\right]+\Gamma_{\theta}\left[\left(\hat{\theta}_{t}^{i}\right)^{2}+\left(\hat{\theta}_{t}^{j}\right)^{2}\right]+\right. \\
& \Gamma_{c}\left[\left(\hat{c}_{t}^{i}\right)^{2}+\left(\hat{c}_{t}^{j}\right)^{2}\right]+\Gamma_{c_{H}}\left[\left(\hat{c}_{H, t}^{i}\right)^{2}+\left(\hat{c}_{H, t}^{j}\right)^{2}\right]+\Gamma_{c_{F}}\left[\left(\hat{c}_{F, t}^{i}\right)^{2}+\left(\hat{c}_{F, t}^{j}\right)^{2}\right]+ \\
& \left.\Gamma_{l}\left[\left(\hat{l}_{t}^{i}\right)^{2}+\left(\hat{l}_{t}^{j}\right)^{2}\right]+\Gamma_{\Delta}\left[\hat{\Delta}_{t}^{i}+\hat{\Delta}_{t}^{j}\right]+2 \Gamma_{a e}\left[\hat{a}_{t}^{i} \hat{e}_{t}^{i}+\hat{a}_{t}^{j} \hat{e}_{t}^{j}\right]\right\}+t . i . p .
\end{aligned}
$$

Appendix F.6. Welfare function

The second-order approximation of the utility function can be rewritten as a sum of squared deviations of endogenous variables from their Pareto efficient level. For a generic variable specific to region $i, X_{t}^{i}$, let us define $\tilde{x}_{t}^{i} \equiv \log X_{t}^{i}-\log X_{t}^{i \star}$, such that $\hat{x}_{t}^{i}=\tilde{x}_{t}^{i}+\hat{x}_{t}^{i \star}$. Such a variable represents the gap between the market solution and the efficient one. It is useful to define the deviation of the efficient allocation for the (efficient) steady state as $\hat{x}_{t}^{i \star} \equiv \log X_{t}^{i \star}-\log X^{i}$. Using these definitions, (F.12) becomes

$$
\begin{aligned}
W \simeq & \frac{1}{2} \mathbf{E}_{\mathbf{0}} \sum_{t=0}^{\infty} \beta^{t}\left\{\Gamma_{e}\left[\left(\tilde{e}_{t}^{i}\right)^{2}+\left(\tilde{e}_{t}^{j}\right)^{2}\right]+\Gamma_{\theta}\left[\left(\tilde{\theta}_{t}^{i}\right)^{2}+\left(\tilde{\theta}_{t}^{j}\right)^{2}\right]+\right. \\
& \Gamma_{c}\left[\left(\tilde{c}_{t}^{i}\right)^{2}+\left(\tilde{c}_{t}^{j}\right)^{2}\right]+\Gamma_{c_{H}}\left[\left(\tilde{c}_{H, t}^{i}\right)^{2}+\left(\tilde{c}_{H, t}^{j}\right)^{2}\right]+\Gamma_{c_{F}}\left[\left(\tilde{c}_{F, t}^{i}\right)^{2}+\left(\tilde{c}_{F, t}^{j}\right)^{2}\right]+ \\
& \left.\Gamma_{l}\left[\left(\tilde{l}_{t}^{i}\right)^{2}+\left(\tilde{l}_{t}^{j}\right)^{2}\right]+\Gamma_{\Delta}\left[\tilde{\Delta}_{t}^{i}+\tilde{\Delta}_{t}^{j}\right]\right\}+ \\
& \mathbf{E}_{\mathbf{0}} \sum_{t=0}^{\infty} \beta^{t}\left\{\Gamma_{e}\left[\tilde{e}_{t}^{i} \hat{e}_{t}^{i \star}+\tilde{e}_{t}^{j} \hat{e}_{t}^{j \star}\right]+\Gamma_{\theta}\left[\tilde{\theta}_{t}^{i} \hat{\theta}_{t}^{i \star}+\tilde{\theta}_{t}^{j} \hat{\theta}_{t}^{j \star}\right]+\right. \\
& \Gamma_{c}\left[\tilde{c}_{t}^{i} \hat{c}_{t}^{i \star}+\tilde{c}_{t}^{j} \hat{c}_{t}^{j \star}\right]+\Gamma_{c_{H}}\left[\tilde{c}_{H, t}^{i} \hat{c}_{H, t}^{i \star}+\tilde{c}_{H, t}^{j} \hat{c}_{H, t}^{j \star}\right]+\Gamma_{c_{F}}\left[\tilde{c}_{F, t}^{i} \hat{c}_{F, t}^{i \star}+\tilde{c}_{F, t}^{j} \hat{c}_{F, t}^{j \star}\right] \\
& \left.+\Gamma_{l}\left[\tilde{l}_{t}^{i} \hat{l}_{t}^{i \star}+\tilde{l}_{t}^{j} \hat{l}_{t}^{j \star}\right]+\Gamma_{a e}\left[\hat{a}_{t}^{i} \hat{e}_{t}^{i}+\hat{a}_{t}^{j} \tilde{e}_{t}^{j}\right]\right\}+t . i \cdot p .
\end{aligned}
$$


Define

$$
\begin{aligned}
A_{0} \equiv & \mathbf{E}_{\mathbf{0}} \sum_{t=0}^{\infty} \beta^{t}\left\{\Gamma_{e}\left[\tilde{e}_{t}^{i} \hat{e}_{t}^{i \star}+\tilde{e}_{t}^{j} \hat{e}_{t}^{j \star}\right]+\Gamma_{\theta}\left[\tilde{\theta}_{t}^{i} \hat{\theta}_{t}^{i \star}+\tilde{\theta}_{t}^{j} \hat{\theta}_{t}^{j \star}\right]+\right. \\
& \Gamma_{c}\left[\tilde{c}_{t}^{i} \hat{c}_{t}^{i \star}+\tilde{c}_{t}^{j} \hat{c}_{t}^{j \star}\right]+\Gamma_{c_{H}}\left[\tilde{c}_{H, t}^{i} \hat{c}_{H, t}^{i \star}+\tilde{c}_{H, t}^{j} \hat{c}_{H, t}^{j \star}\right]+\Gamma_{c_{F}}\left[\tilde{c}_{F, t}^{i} \hat{c}_{F, t}^{i \star}+\tilde{c}_{F, t}^{j} \hat{c}_{F, t}^{j \star}\right] \\
& \left.+\Gamma_{l}\left[\tilde{l}_{t}^{i} \hat{l}_{t}^{i \star}+\tilde{l}_{t}^{j} \hat{l}_{t}^{j \star}\right]+\Gamma_{a e}\left[\hat{a}_{t}^{i} \tilde{e}_{t}^{i}+\hat{a}_{t}^{j} \tilde{e}_{t}^{j}\right]\right\}+t . i . p .
\end{aligned}
$$

One can show that around the efficient steady state, the sum of all cross-products is equal to zero, i.e. $A_{0}=0$, such that only squared deviations are left in the welfare function. First, recall that both feasibility constraints hold in the market and in the efficient equilibrium, such that

$$
\begin{aligned}
& \mathbf{E}_{\mathbf{0}} \sum_{t=0}^{\infty} \beta^{t} \Gamma_{c} \tilde{c}_{t}^{i} \hat{c}_{t}^{i \star}= \\
& \mathbf{E}_{\mathbf{0}} \sum_{t=0}^{\infty} \beta^{t}\left\{-\frac{E}{C} \frac{\nu_{2}-1}{\nu_{2}}\left[\frac{1-\alpha}{E^{\alpha}}-\frac{\kappa}{q}\right] \tilde{e}_{t}^{i} \hat{c}_{t}^{i \star}+\frac{E}{C} \frac{\nu_{2}-1}{\nu_{2}} \frac{\kappa}{q}(1-\gamma) \rho \tilde{\theta}_{t}^{i} c_{t}^{i \star}-\right. \\
& \left.\frac{E}{C} \frac{\nu_{2}-1}{\nu_{2}} \beta \frac{\kappa}{q}(1-\rho) \tilde{e}_{t}^{i} \hat{c}_{t+1}^{i \star}-\alpha_{2} \frac{\nu_{2}-1}{\nu_{2}} \tilde{c}_{F, t}^{i} \hat{c}_{t}^{i \star}+\alpha_{2} \frac{\nu_{2}-1}{\nu_{2}} \tilde{c}_{F, t}^{j} \hat{c}_{t}^{i \star}\right\}+t . i . p .
\end{aligned}
$$

$\mathbf{E}_{\mathbf{0}} \sum_{t=0}^{\infty} \beta^{t} \Gamma_{l} \tilde{l}_{t}^{i} \hat{l}_{t}^{i \star}=$

$\mathbf{E}_{\mathbf{0}} \sum_{t=0}^{\infty} \beta^{t} \frac{1-b}{b} \frac{E}{l}\left\{\left(1+\zeta \frac{1-f}{f}\right) \tilde{e}_{t}^{i} \hat{l}_{t}^{i^{i}}-\frac{\rho \zeta \gamma}{f} \tilde{\theta}_{t}^{i} \hat{l}_{t}^{i \star}-\beta(1-\rho) \zeta \frac{1-f}{f} \tilde{e}_{t}^{i} \hat{l}_{t+1}^{i^{\star}}\right\}+t . i . p$.

Therefore, (F.14) can be rewritten, using (F.15), (F.16), and the respective expressions for region $j$ :

$$
\begin{aligned}
& A_{0}=\mathbf{E}_{\mathbf{0}} \sum_{t=0}^{\infty} \beta^{t}\left\{\tilde{e}_{t}^{i} \frac{E}{C} \frac{1}{\nu_{2}}\left[\left(\frac{1-\alpha}{E^{\alpha}}-\frac{\kappa}{q}\right) \hat{c}_{H, t}^{i \star}+\beta(1-\rho) \frac{\kappa}{q} \hat{c}_{H, t+1}^{i \star}\right]+\right. \\
& \tilde{e}_{t}^{j} \frac{E}{C} \frac{1}{\nu_{2}}\left[\left(\frac{1-\alpha}{E^{\alpha}}-\frac{\kappa}{q}\right) \hat{c}_{H, t}^{j \star}+\beta(1-\rho) \frac{\kappa}{q} \hat{c}_{H, t+1}^{j \star}\right]- \\
& \tilde{\theta}_{t}^{i}\left[\frac{E}{C} \frac{\kappa}{q} \rho(1-\gamma) \frac{1}{\nu_{2}} \hat{c}_{H, t}^{i \star}\right]-\tilde{\theta}_{t}^{j}\left[\frac{E}{C} \frac{\kappa}{q} \rho(1-\gamma) \frac{1}{\nu_{2}} \hat{c}_{H, t}^{j \star}\right]- \\
& \left.\left(1-\alpha_{2}\right)\left[\tilde{c}_{H, t}^{i} \hat{c}_{H, t}^{i \star}+\tilde{c}_{H, t}^{j} \hat{c}_{H, t}^{j \star}\right]-\alpha_{2}\left[\tilde{c}_{F, t}^{j} \hat{c}_{H, t}^{i \star}+\tilde{c}_{F, t}^{i} \hat{c}_{H, t}^{j \star}\right]\right\}+t . i . p .,
\end{aligned}
$$

where, by the feasibility constraint (F.4),

$$
\begin{aligned}
-\hat{c}_{H, t}^{i \star}\left[\left(1-\alpha_{2}\right) \tilde{c}_{H, t}^{i}+\alpha_{2} \tilde{c}_{F, t}^{j}\right]= & \tilde{e}_{t}^{i} \frac{E}{C} \frac{1}{\nu_{2}}\left[\left(\frac{1-\alpha}{E^{\alpha}}-\frac{\kappa}{q}\right) \hat{c}_{H, t}^{i \star}+\beta(1-\rho) \frac{\kappa}{q} \hat{c}_{H, t+1}^{i \star}\right]- \\
& \tilde{\theta}_{t}^{i}\left[\frac{E}{C} \frac{\kappa}{q} \rho(1-\gamma) \frac{1}{\nu_{2}} \hat{c}_{H, t}^{i \star}\right],
\end{aligned}
$$


such that

$$
A_{0}=0 .
$$

From Woodford (2011) we know that

$$
\sum_{t=0}^{\infty} \beta^{t} \operatorname{Var}_{l} \log \left(P_{H, t}^{i}(l)\right)=\sum_{t=0}^{\infty} \beta^{t} \frac{\delta}{(1-\delta)(1-\beta \delta)}\left(\hat{\pi}_{H, t}^{i}\right)^{2},
$$

so that

$$
\sum_{t=0}^{\infty} \beta^{t} \Gamma_{\Delta} \tilde{\Delta}_{t}^{i}=\sum_{t=0}^{\infty} \beta^{t} \Gamma_{\pi_{H}^{i}}\left(\hat{\pi}_{H, t}^{i}\right)^{2}=\sum_{t=0}^{\infty} \beta^{t} \Gamma_{\pi_{H}^{i}}\left(\tilde{\pi}_{H, t}^{i}\right)^{2}
$$

where

$$
\Gamma_{\pi_{H}^{i}} \equiv-\frac{\varepsilon}{\lambda^{i}} \frac{E^{1-\alpha}}{C}=-\frac{\varepsilon}{\lambda^{i}} \frac{Y}{C} .
$$

Therefore, a second-order approximation to the utility function simply yields

$$
\begin{aligned}
& W \simeq \frac{1}{2} \mathbf{E}_{\mathbf{0}} \sum_{t=0}^{\infty} \beta^{t}\left\{\Gamma_{e}\left[\left(\tilde{e}_{t}^{i}\right)^{2}+\left(\tilde{e}_{t}^{j}\right)^{2}\right]+\Gamma_{\theta}\left[\left(\tilde{\theta}_{t}^{i}\right)^{2}+\left(\tilde{\theta}_{t}^{j}\right)^{2}\right]+\right. \\
& \Gamma_{c}\left[\left(\tilde{c}_{t}^{i}\right)^{2}+\left(\tilde{c}_{t}^{j}\right)^{2}\right]+\Gamma_{c_{H}}\left[\left(\tilde{c}_{H, t}^{i}\right)^{2}+\left(\tilde{c}_{H, t}^{j}\right)^{2}\right]+\Gamma_{c_{F}}\left[\left(\tilde{c}_{F, t}^{i}\right)^{2}+\left(\tilde{c}_{F, t}^{j}\right)^{2}\right]+ \\
& \left.\Gamma_{l}\left[\left(\tilde{l}_{t}^{i}\right)^{2}+\left(\tilde{l}_{t}^{j}\right)^{2}\right]+\Gamma_{\pi_{H}^{i}}\left(\tilde{\pi}_{H, t}^{i}\right)^{2}+\Gamma_{\pi_{H}^{j}}\left(\tilde{\pi}_{H, t}^{j}\right)^{2}\right\}+t . i . p .
\end{aligned}
$$




\section{Appendix G. A purely quadratic welfare criterion-Full model with labor mobility}

As for the benchmark model without labor mobility in Appendix F, we approximate households' utility function to the second order, substitute out the linear terms in consumption and leisure using the respective feasibility constraints, and show that our welfare criterion can be rewritten in purely quadratic terms.

Appendix G.1. Taylor expansion of the utility function and feasibility constraints

As for the benchmark model with a fixed labor force, a second-order log-linear approximation of the instantaneous utility function in region $i$, for the particular case where $\sigma=1$, states as

$$
\frac{U_{t}^{i}-U^{i}}{U_{c}^{i}} \simeq \hat{c}_{t}^{i}+\frac{1-b}{b} \hat{l}_{t}^{i}+t . i . p .
$$

The consumption aggregator for region $i$, up to second order, reads as

$\hat{c}_{t}^{i}=-\frac{1}{2} \frac{\nu_{2}-1}{\nu_{2}}\left(\hat{c}_{t}^{i}\right)^{2}+\left(1-\alpha_{2}\right)\left[\hat{c}_{H, t}^{i}+\frac{1}{2} \frac{\nu_{2}-1}{\nu_{2}}\left(\hat{c}_{H, t}^{i}\right)^{2}\right]+\alpha_{2}\left[\hat{c}_{F, t}^{i}+\frac{1}{2} \frac{\nu_{2}-1}{\nu_{2}}\left(\hat{c}_{F, t}^{i}\right)^{2}\right]$,

while the first feasibility constraint for region $i$,

$$
\begin{aligned}
\left(\frac{A_{t}^{i}\left(E_{t}^{i i}+E_{t}^{j i}\right)}{\Delta_{t}^{i}}\right)^{(1-\alpha)}= & C_{H, t}^{i}+C_{F, t}^{j}+\frac{\kappa}{\tau}\left(\theta_{t}^{i i}\right)^{1-\gamma}\left[E_{t}^{i i}-\left(1-\rho^{i}\right) E_{t-1}^{i i}\right] \\
& +\frac{\kappa}{\tau}\left(\theta_{t}^{j i}\right)^{1-\gamma}\left[E_{t}^{j i}-\left(1-\rho^{i}\right) E_{t-1}^{j i}\right],
\end{aligned}
$$

up to the second order, around the symmetric steady state, states as

$$
\begin{aligned}
& (1-\alpha) E^{1-\alpha}\left[-\hat{\Delta}_{t}^{i}+\left(1-\alpha_{1}\right) \hat{e}_{t}^{i i}+\alpha_{1} \hat{e}_{t}^{j i}+\frac{1}{2}\left[1-\alpha\left(1-\alpha_{1}\right)\right]\left(1-\alpha_{1}\right)\left(\hat{e}_{t}^{i i}\right)^{2}+\right. \\
& \left.\frac{1}{2}\left[1-\alpha \alpha_{1}\right] \alpha_{1}\left(\hat{e}_{t}^{j i}\right)^{2}+(1-\alpha)\left(1-\alpha_{1}\right) \hat{a}_{t}^{i} \hat{e}_{t}^{i i}+(1-\alpha) \alpha_{1} \hat{a}_{t}^{i} \hat{e}_{t}^{j i}-\alpha\left(1-\alpha_{1}\right) \alpha_{1} \hat{e}_{t}^{i i} \hat{e}_{t}^{j i}\right]= \\
& C_{H}\left[\hat{c}_{H, t}^{i}+\frac{1}{2}\left(\hat{c}_{H, t}^{i}\right)^{2}\right]+C_{F}\left[\hat{c}_{F, t}^{j}+\frac{1}{2}\left(\hat{c}_{F, t}^{j}\right)^{2}\right]+ \\
& \frac{\kappa}{q}\left(1-\alpha_{1}\right) E\left[(1-\gamma) \hat{\theta}_{t}^{i i}+\hat{e}_{t}^{i i}+\frac{1}{2}(1-\gamma)^{2}\left(\hat{\theta}_{t}^{i i}\right)^{2}+\frac{1}{2}\left(\hat{e}_{t}^{i i}\right)^{2}+(1-\gamma) \hat{\theta}_{t}^{i i} \hat{e}_{t}^{i i}\right]- \\
& \frac{\kappa}{q}\left(1-\alpha_{1}\right) E(1-\rho)\left[(1-\gamma) \hat{\theta}_{t}^{i i}+\hat{e}_{t-1}^{i i}+\frac{1}{2}(1-\gamma)^{2}\left(\hat{\theta}_{t}^{i i}\right)^{2}+\frac{1}{2}\left(\hat{e}_{t-1}^{i i}\right)^{2}+(1-\gamma) \hat{\theta}_{t}^{i i} \hat{e}_{t-1}^{i i}\right]+ \\
& \frac{\kappa}{q} \alpha_{1} E\left[(1-\gamma) \hat{\theta}_{t}^{j i}+\hat{e}_{t}^{j i}+\frac{1}{2}(1-\gamma)^{2}\left(\hat{\theta}_{t}^{j i}\right)^{2}+\frac{1}{2}\left(\hat{e}_{t}^{j i}\right)^{2}+(1-\gamma) \hat{\theta}_{t}^{j i} \hat{e}_{t}^{j i}\right]- \\
& \frac{\kappa}{q} \alpha_{1} E(1-\rho)\left[(1-\gamma) \hat{\theta}_{t}^{j i}+\hat{e}_{t-1}^{j i}+\frac{1}{2}(1-\gamma)^{2}\left(\hat{\theta}_{t}^{j i}\right)^{2}+\frac{1}{2}\left(\hat{e}_{t-1}^{j i}\right)^{2}+(1-\gamma) \hat{\theta}_{t}^{j i} \hat{e}_{t-1}^{j i}\right]
\end{aligned}
$$


The above expression can then be rewritten as follows:

$$
\begin{aligned}
& \hat{c}_{H, t}^{i}=\frac{(1-\alpha) E^{1-\alpha}}{C_{H}}\left[-\hat{\Delta}_{t}^{i}+\left(1-\alpha_{1}\right) \hat{e}_{t}^{i i}+\alpha_{1} \hat{e}_{t}^{j i}+\frac{1}{2}\left[1-\alpha\left(1-\alpha_{1}\right)\right]\left(1-\alpha_{1}\right)\left(\hat{e}_{t}^{i i}\right)^{2}+\right. \\
& \left.\frac{1}{2}\left[1-\alpha \alpha_{1}\right] \alpha_{1}\left(\hat{e}_{t}^{j i}\right)^{2}+(1-\alpha)\left(1-\alpha_{1}\right) \hat{a}_{t}^{i} \hat{e}_{t}^{i i}+(1-\alpha) \alpha_{1} \hat{a}_{t}^{i} \hat{e}_{t}^{j i}-\alpha\left(1-\alpha_{1}\right) \alpha_{1} \hat{e}_{t}^{i i} \hat{e}_{t}^{j i}\right]- \\
& \frac{\kappa}{q} \frac{\left(1-\alpha_{1}\right) E}{C_{H}}\left[(1-\gamma) \hat{\theta}_{t}^{i i}+\hat{e}_{t}^{i i}+\frac{1}{2}(1-\gamma)^{2}\left(\hat{\theta}_{t}^{i i}\right)^{2}+\frac{1}{2}\left(\hat{e}_{t}^{i i}\right)^{2}+(1-\gamma) \hat{\theta}_{t}^{i i} \hat{e}_{t}^{i i}\right]+ \\
& \frac{\kappa}{q} \frac{\left(1-\alpha_{1}\right) E}{C_{H}}(1-\rho)\left[(1-\gamma) \hat{\theta}_{t}^{i i}+\hat{e}_{t-1}^{i i}+\frac{1}{2}(1-\gamma)^{2}\left(\hat{\theta}_{t}^{i i}\right)^{2}+\frac{1}{2}\left(\hat{e}_{t-1}^{i i}\right)^{2}+(1-\gamma) \hat{\theta}_{t}^{i i} \hat{e}_{t-1}^{i i}\right]- \\
& \frac{\kappa}{q} \frac{\alpha_{1} E}{C_{H}}\left[(1-\gamma) \hat{\theta}_{t}^{j i}+\hat{e}_{t}^{j i}+\frac{1}{2}(1-\gamma)^{2}\left(\hat{\theta}_{t}^{j i}\right)^{2}+\frac{1}{2}\left(\hat{e}_{t}^{j i}\right)^{2}+(1-\gamma) \hat{\theta}_{t}^{j i} \hat{e}_{t}^{j i}\right]+ \\
& \frac{\kappa}{q} \frac{\alpha_{1} E}{C_{H}}(1-\rho)\left[(1-\gamma) \hat{\theta}_{t}^{j i}+\hat{e}_{t-1}^{j i}+\frac{1}{2}(1-\gamma)^{2}\left(\hat{\theta}_{t}^{j i}\right)^{2}+\frac{1}{2}\left(\hat{e}_{t-1}^{j i}\right)^{2}+(1-\gamma) \hat{\theta}_{t}^{j i} \hat{e}_{t-1}^{j i}\right]- \\
& \frac{1}{2}\left(\hat{c}_{H, t}^{i}\right)^{2}-\frac{\alpha_{2}}{1-\alpha_{2}}\left[\hat{c}_{F, t}^{j}+\frac{1}{2}\left(\hat{c}_{F, t}^{j}\right)^{2}\right] .
\end{aligned}
$$

Combining (G.2) and (G.5) yields

$$
\begin{aligned}
& \hat{c}_{t}^{i}=-\frac{1}{2} \frac{\nu_{2}-1}{\nu_{2}}\left(\hat{c}_{t}^{i}\right)^{2}+\frac{1}{2} \frac{\nu_{2}-1}{\nu_{2}}\left(1-\alpha_{2}\right)\left(\hat{c}_{H, t}^{i}\right)^{2}+\alpha_{2}\left[\hat{c}_{F, t}^{i}+\frac{1}{2} \frac{\nu_{2}-1}{\nu_{2}}\left(\hat{c}_{F, t}^{i}\right)^{2}\right]+ \\
& \frac{(1-\alpha) E^{1-\alpha}}{C}\left[-\hat{\Delta}_{t}^{i}+\left(1-\alpha_{1}\right) \hat{e}_{t}^{i i}+\alpha_{1} \hat{e}_{t}^{j i}+\frac{1}{2}\left[1-\alpha\left(1-\alpha_{1}\right)\right]\left(1-\alpha_{1}\right)\left(\hat{e}_{t}^{i i}\right)^{2}+\right. \\
& \left.\frac{1}{2}\left[1-\alpha \alpha_{1}\right] \alpha_{1}\left(\hat{e}_{t}^{j i}\right)^{2}+(1-\alpha)\left(1-\alpha_{1}\right) \hat{a}_{t}^{i} \hat{e}_{t}^{i i}+(1-\alpha) \alpha_{1} \hat{a}_{t}^{i} \hat{e}_{t}^{j i}-\alpha\left(1-\alpha_{1}\right) \alpha_{1} \hat{e}_{t}^{i i} \hat{e}_{t}^{j i}\right]- \\
& \frac{\kappa}{q} \frac{E}{C}\left(1-\alpha_{1}\right)\left[(1-\gamma) \hat{\theta}_{t}^{i i}+\hat{e}_{t}^{i i}+\frac{1}{2}(1-\gamma)^{2}\left(\hat{\theta}_{t}^{i i}\right)^{2}+\frac{1}{2}\left(\hat{e}_{t}^{i i}\right)^{2}+(1-\gamma) \hat{\theta}_{t}^{i i} \hat{e}_{t}^{i i}\right]+ \\
& \frac{\kappa}{q} \frac{E}{C}\left(1-\alpha_{1}\right)(1-\rho)\left[(1-\gamma) \hat{\theta}_{t}^{i i}+\hat{e}_{t-1}^{i i}+\frac{1}{2}(1-\gamma)^{2}\left(\hat{\theta}_{t}^{i i}\right)^{2}+\frac{1}{2}\left(\hat{e}_{t-1}^{i i}\right)^{2}+(1-\gamma) \hat{\theta}_{t}^{i i} \hat{e}_{t-1}^{i i}\right]- \\
& \frac{\kappa}{q} \frac{E}{C} \alpha_{1}\left[(1-\gamma) \hat{\theta}_{t}^{j i}+\hat{e}_{t}^{j i}+\frac{1}{2}(1-\gamma)^{2}\left(\hat{\theta}_{t}^{j i}\right)^{2}+\frac{1}{2}\left(\hat{e}_{t}^{j i}\right)^{2}+(1-\gamma) \hat{\theta}_{t}^{j i} \hat{e}_{t}^{j i}\right]+ \\
& \frac{\kappa}{q} \frac{E}{C} \alpha_{1}(1-\rho)\left[(1-\gamma) \hat{\theta}_{t}^{j i}+\hat{e}_{t-1}^{j i}+\frac{1}{2}(1-\gamma)^{2}\left(\hat{\theta}_{t}^{j i}\right)^{2}+\frac{1}{2}\left(\hat{e}_{t-1}^{j i}\right)^{2}+(1-\gamma) \hat{\theta}_{t}^{j i} \hat{e}_{t-1}^{j i}\right]- \\
& \frac{1}{2}\left(1-\alpha_{2}\right)\left(\hat{c}_{H, t}^{i}\right)^{2}-\alpha_{2}\left[\hat{c}_{F, t}^{j}+\frac{1}{2}\left(\hat{c}_{F, t}^{j}\right)^{2}\right] .
\end{aligned}
$$

An equivalent expression for total consumption in region $j, \hat{c}_{t}^{j}$, can be derived by combining region $j$ 's consumption aggregator with its feasibility constraint. The second 
feasibility constraint for region $i$ relates to households' time allocation,

$$
\begin{array}{rr}
\left(1-l_{t}^{i}\right)^{\frac{\nu_{1}-1}{\nu_{1}}}= & \left(1-\alpha_{1}\right)^{1 / \nu_{1}}\left[E_{t}^{i i}+\zeta U_{t}^{i i}\right]^{\frac{\nu_{1}-1}{\nu_{1}}}+\alpha_{1}^{1 / \nu_{1}}\left[E_{t}^{i j}+\zeta U_{t}^{i j}\right]^{\frac{\nu_{1}-1}{\nu_{1}}} \\
= & \left(1-\alpha_{1}\right)^{1 / \nu_{1}}\left[E_{t}^{i i}+\zeta \frac{1-\tau\left(\theta_{t}^{i i}\right)^{\gamma}}{\tau\left(\theta_{t}^{i i}\right)^{\gamma}} E_{t}^{i i}-\zeta(1-\rho) \frac{1-\tau\left(\theta_{t}^{i i}\right)^{\gamma}}{\tau\left(\theta_{t}^{i i}\right)^{\gamma}} E_{t-1}^{i i}\right]^{\frac{\nu_{1}-1}{\nu_{1}}}+ \\
\alpha_{1}^{1 / \nu_{1}}\left[E_{t}^{i j}+\zeta \frac{1-\tau\left(\theta_{t}^{i j}\right)^{\gamma}}{\tau\left(\theta_{t}^{i j}\right)^{\gamma}} E_{t}^{i j}-\zeta(1-\rho) \frac{1-\tau\left(\theta_{t}^{i j}\right)^{\gamma}}{\tau\left(\theta_{t}^{i j}\right)^{\gamma}} E_{t-1}^{i j}\right]^{\frac{\nu_{1}-1}{\nu_{1}}}
\end{array}
$$

which, up to second order, states as

$$
\begin{aligned}
& \hat{l}_{t}^{i}=-\frac{1}{2}\left(1+\frac{1}{\nu_{1}} \frac{l}{1-l}\right)\left(\hat{l}_{t}^{i}\right)^{2}-\left(1-\alpha_{1}\right) \frac{E}{l}\left(1+\zeta \frac{1-f}{f}\right) \hat{e}_{t}^{i i}-\alpha_{1} \frac{E}{l}\left(1+\zeta \frac{1-f}{f}\right) \hat{e}_{t}^{i j} \\
& +\left(1-\alpha_{1}\right) \frac{E}{l} \frac{\rho \zeta \gamma}{f} \hat{\theta}_{t}^{i i}+\alpha_{1} \frac{E}{l} \frac{\rho \zeta}{f} \hat{\theta}_{t}^{i j} \\
& +\left(1-\alpha_{1}\right) \frac{E}{l}(1-\rho) \zeta \frac{1-f}{f} \hat{e}_{t-1}^{i i}+\alpha_{1} \frac{E}{l}(1-\rho) \zeta \frac{1-f}{f} \hat{e}_{t-1}^{i j} \\
& -\frac{1}{2}\left(1-\alpha_{1}\right) \frac{E}{l}\left(1+\zeta \frac{1-f}{f}\right)\left(1-\frac{1}{\nu_{1}} \frac{f+\zeta(1-f)}{f+\zeta \rho(1-f)}\right)\left(\hat{e}_{t}^{i i}\right)^{2} \\
& -\frac{1}{2} \alpha_{1} \frac{E}{l}\left(1+\zeta \frac{1-f}{f}\right)\left(1-\frac{1}{\nu_{1}} \frac{f+\zeta(1-f)}{f+\zeta \rho(1-f)}\right)\left(\hat{e}_{t}^{i j}\right)^{2} \\
& -\frac{1}{2}\left(1-\alpha_{1}\right) \frac{E}{l} \frac{\rho \gamma^{2} \zeta}{f}\left(1-\frac{1}{\nu_{1}} \frac{\zeta \rho}{f+\zeta \rho(1-f)}\right)\left(\hat{\theta}_{t}^{i i}\right)^{2} \\
& -\frac{1}{2} \alpha_{1} \frac{E}{l} \frac{\rho \gamma^{2} \zeta}{f}\left(1-\frac{1}{\nu_{1}} \frac{\zeta \rho}{f+\zeta \rho(1-f)}\right)\left(\hat{\theta}_{t}^{i j}\right)^{2} \\
& +\frac{1}{2}\left(1-\alpha_{1}\right) \frac{E}{l}(1-\rho) \zeta \frac{1-f}{f}\left(1+\frac{1}{\nu_{1}} \frac{\zeta(1-\rho)(1-f)}{f+\zeta \rho(1-f)}\right)^{\left(\hat{e}_{t-1}^{i i}\right)^{2}} \\
& +\frac{1}{2} \alpha_{1} \frac{E}{l}(1-\rho) \zeta \frac{1-f}{f}\left(1+\frac{1}{\nu_{1}} \frac{\zeta(1-\rho)(1-f)}{f+\zeta \rho(1-f)}\right)\left(\hat{e}_{t-1}^{i j}\right)^{2} \\
& +\frac{\zeta \gamma}{f}\left(1-\alpha_{1}\right) \frac{E}{l} \hat{\theta}_{t}^{i i}\left[\hat{e}_{t}^{i i}\left(1-\frac{1}{\nu_{1}} \frac{\rho(f+\zeta(1-f))}{f+\zeta(1-f) \rho}\right)-(1-\rho) \hat{e}_{t-1}^{i i}\left(1-\frac{1}{\nu_{1}} \frac{\rho \zeta(1-\zeta(1-f) \rho}{f+f}\right)\right] \\
& +\frac{\zeta \gamma}{f} \alpha_{1} \frac{E}{l} \hat{\theta}_{t}^{i j}\left[\hat{e}_{t}^{i j}\left(1-\frac{1}{\nu_{1}} \frac{\rho(f+\zeta(1-f))}{f+\zeta(1-f) \rho}\right)-(1-\rho) \hat{e}_{t-1}^{i j}\left(1-\frac{1}{\nu_{1}} \frac{\rho \zeta(1-f)}{f+\zeta(1-f) \rho}\right)\right] \\
& +t . i . p .
\end{aligned}
$$

The instantaneous utility functions of the two regions constituting a monetary union, approximated to second order around a symmetric steady state, therefore read as

$$
\left[\hat{c}_{t}^{i}+\frac{1-b}{b} \hat{l}_{t}^{i}\right]+\left[\hat{c}_{t}^{j}+\frac{1-b}{b} \hat{l}_{t}^{j}\right]+\text { t.i.p. }
$$


where $\hat{c}_{t}^{i}$ and $\hat{l}_{t}^{i}$ are given by equations (G.6) and (G.7), respectively. Equivalent equations can be derived for consumption and leisure in region $j$.

\section{Appendix G.2. Linear terms}

One can then collect all linear terms appearing in the generic period $t$ so that all first-order terms in the approximated discounted and weighted lifetime utility of the representative household in both regions read as

$$
\begin{aligned}
& \sum_{t=0}^{\infty} \beta^{t}\left[\Lambda_{1}^{i i}\left(\hat{e}_{t}^{i i}+\hat{e}_{t}^{j j}\right)+\Lambda_{1}^{i j}\left(\hat{e}_{t}^{i j}+\hat{e}_{t}^{j i}\right)+\Lambda_{2}^{i i}\left(\hat{\theta}_{t}^{i i}+\hat{\theta}_{t}^{j j}\right)+\Lambda_{2}^{i j}\left(\hat{\theta}_{t}^{i j}+\hat{\theta}_{t}^{j i}\right)+\right. \\
& \left.\Lambda_{3}^{i i}\left(\hat{e}_{t-1}^{i i}+\hat{e}_{t-1}^{j j}\right)+\Lambda_{3}^{i j}\left(\hat{e}_{t-1}^{i j}+\hat{e}_{t-1}^{j i}\right)+\Lambda_{4}\left(\hat{c}_{F, t}^{i}+\hat{c}_{F, t}^{j}\right)\right]= \\
& \Lambda_{3}^{i i}\left(\hat{e}_{-1}^{i i}+\hat{e}_{-1}^{j j}\right)+\Lambda_{3}^{i j}\left(\hat{e}_{-1}^{i j}+\hat{e}_{-1}^{j i}\right)+\sum_{t=0}^{\infty} \beta^{t}\left[\left(\Lambda_{1}^{i i}+\beta \Lambda_{3}^{i i}\right)\left(\hat{e}_{t}^{i i}+\hat{e}_{t}^{j j}\right)+\right. \\
& \left.\left(\Lambda_{1}^{i j}+\beta \Lambda_{3}^{i j}\right)\left(\hat{e}_{t}^{i j}+\hat{e}_{t}^{j i}\right)+\Lambda_{2}^{i i}\left(\hat{\theta}_{t}^{i i}+\hat{\theta}_{t}^{j j}\right)+\Lambda_{2}^{i j}\left(\hat{\theta}_{t}^{i j}+\hat{\theta}_{t}^{j i}\right)+\Lambda_{4}\left(\hat{c}_{F, t}^{i}+\hat{c}_{F, t}^{j}\right)\right],
\end{aligned}
$$

where the terms $\hat{e}_{-1}^{i i}, \hat{e}_{-1}^{j j}, \hat{e}_{-1}^{i j}$, and $\hat{e}_{-1}^{j i}$ are given at time $t=0$ and are thus to be considered independent of policy. At the symmetric steady state, the coefficients on native employment states as follows:

$$
\begin{gathered}
\Lambda_{1}^{i i}+\beta \Lambda_{3}^{i i}= \\
\left(1-\alpha_{1}\right) \frac{E}{C}\{\underbrace{\frac{1-\alpha}{E^{\alpha}}-\frac{\kappa}{q}[1-\beta(1-\rho)]-\frac{1-b}{b} \frac{C}{l}\left[1+\zeta\left(\frac{1-f}{f}\right)(1-\beta(1-\rho))\right]}_{=0, \text { by steady-state efficiency condition (E.2) }}\} .
\end{gathered}
$$

Equivalently, the coefficient on migrant employment reads as follows:

$$
\begin{gathered}
\Lambda_{1}^{i j}+\beta \Lambda_{3}^{i j}= \\
=\alpha_{1} \frac{E}{C}\{\underbrace{\frac{1-\alpha}{E^{\alpha}}-\frac{\kappa}{q}[1-\beta(1-\rho)]-\frac{1-b}{b} \frac{C}{l}\left[1+\zeta\left(\frac{1-f}{f}\right)(1-\beta(1-\rho))\right]}_{=0, \text { by steady-state efficiency condition (E.2) }}\}
\end{gathered}
$$

The coefficient on labor market tightness of native workers is given by

$$
\Lambda_{2}^{i i}=\left(1-\alpha_{1}\right) \frac{E}{C} \rho \frac{\gamma}{f}[\underbrace{\frac{1-b}{b} \frac{C}{l} \zeta-\frac{1-\gamma}{\gamma} \kappa \theta}_{=0, \text { by (E.1) }}] \text {. }
$$


Similarly, the coefficient on migrants' labor market tightness

$$
\Lambda_{2}^{i j}=\alpha_{1} \frac{E}{C} \rho \frac{\gamma}{f}[\underbrace{\frac{1-b}{b} \frac{C}{l} \zeta-\frac{1-\gamma}{\gamma} \kappa \theta}_{=0, \text { by (E. } 1)}] .
$$

Finally, as in a standard open-economy model without a mobile labor force, the coefficient on imported consumption is zero, i.e. $\Lambda_{4}=\alpha_{2}-\alpha_{2}=0$. Hence, all linear terms cancel out, and we are left with quadratic terms only. 
Appendix G.3. Quadratic terms for endogenous variables Collecting the purely quadratic terms yields

$$
\begin{aligned}
& \frac{1}{2} \sum_{t=0}^{\infty} \beta^{t}\left\{\Gamma_{1}^{i i}\left[\left(\hat{e}_{t}^{i i}\right)^{2}+\left(\hat{e}_{t}^{j j}\right)^{2}\right]+\Gamma_{1}^{i j}\left[\left(\hat{e}_{t}^{i j}\right)^{2}+\left(\hat{e}_{t}^{j i}\right)^{2}\right]+\right. \\
& \Gamma_{2}^{i i}\left[\left(\hat{e}_{t-1}^{i i}\right)^{2}+\left(\hat{e}_{t-1}^{j j}\right)^{2}\right]+\Gamma_{2}^{i j}\left[\left(\hat{e}_{t-1}^{i j}\right)^{2}+\left(\hat{e}_{t-1}^{j i}\right)^{2}\right]+ \\
& \Gamma_{\theta}^{i i}\left[\left(\hat{\theta}_{t}^{i i}\right)^{2}+\left(\hat{\theta}_{t}^{j j}\right)^{2}\right]+\Gamma_{\theta}^{i j}\left[\left(\hat{\theta}_{t}^{i j}\right)^{2}+\left(\hat{\theta}_{t}^{j i}\right)^{2}\right]+ \\
& \Gamma_{c}\left[\left(\hat{c}_{t}^{i}\right)^{2}+\left(\hat{c}_{t}^{j}\right)^{2}\right]+\Gamma_{c_{H}}\left[\left(\hat{c}_{H, t}^{i}\right)^{2}+\left(\hat{c}_{H, t}^{j}\right)^{2}\right]+\Gamma_{c_{F}}\left[\left(\hat{c}_{F, t}^{i}\right)^{2}+\left(\hat{c}_{F, t}^{j}\right)^{2}\right]+ \\
& \Gamma_{l}\left[\left(\hat{l}_{t}^{i}\right)^{2}+\left(\hat{l}_{t}^{j}\right)^{2}\right]+\Gamma_{\Delta}\left[\hat{\Delta}_{t}^{i}+\hat{\Delta}_{t}^{j}\right]+ \\
& 2 \Gamma_{3}^{i i}\left\{\hat{\theta}_{t}^{i i}\left[\hat{e}_{t}^{i i} \Gamma_{4}-(1-\rho) \hat{e}_{t-1}^{i i}\right]+\hat{\theta}_{t}^{j j}\left[\hat{e}_{t}^{j j} \Gamma_{4}-(1-\rho) \hat{e}_{t-1}^{j j}\right]\right\}+ \\
& 2 \Gamma_{3}^{i j}\left\{\hat{\theta}_{t}^{i j}\left[\hat{e}_{t}^{i j} \Gamma_{4}-(1-\rho) \hat{e}_{t-1}^{i j}\right]+\hat{\theta}_{t}^{j i}\left[\hat{e}_{t}^{j i} \Gamma_{4}-(1-\rho) \hat{e}_{t-1}^{j i}\right]\right\}+ \\
& \left.2 \Gamma_{e^{i i} e^{j i}}\left[\hat{e}_{t}^{i i} \hat{e}_{t}^{j i}+\hat{e}_{t}^{j j} \hat{e}_{t}^{i j}\right]\right\} \\
& =[\underbrace{\Gamma_{2}^{i i}\left[\left(\hat{e}_{-1}^{i i}\right)^{2}+\left(\hat{e}_{-1}^{j j}\right)^{2}\right]+\Gamma_{2}^{i j}\left[\left(\hat{e}_{-1}^{i j}\right)^{2}+\left(\hat{e}_{-1}^{j i}\right)^{2}\right]}_{\text {t.i.p. }}]+ \\
& \frac{1}{2} \sum_{t=0}^{\infty} \beta^{t}\left\{\left(\Gamma_{1}^{i i}+\beta \Gamma_{2}^{i i}\right)\left[\left(\hat{e}_{t}^{i i}\right)^{2}+\left(\hat{e}_{t}^{j j}\right)^{2}\right]+\left(\Gamma_{1}^{i j}+\beta \Gamma_{2}^{i j}\right)\left[\left(\hat{e}_{t}^{i j}\right)^{2}+\left(\hat{e}_{t}^{j i}\right)^{2}\right]+\right. \\
& \Gamma_{\theta}^{i i}\left[\left(\hat{\theta}_{t}^{i i}\right)^{2}+\left(\hat{\theta}_{t}^{j j}\right)^{2}\right]+\Gamma_{\theta}^{i j}\left[\left(\hat{\theta}_{t}^{i j}\right)^{2}+\left(\hat{\theta}_{t}^{j i}\right)^{2}\right]+ \\
& \Gamma_{c}\left[\left(\hat{c}_{t}^{i}\right)^{2}+\left(\hat{c}_{t}^{j}\right)^{2}\right]+\Gamma_{c_{H}}\left[\left(\hat{c}_{H, t}^{i}\right)^{2}+\left(\hat{c}_{H, t}^{j}\right)^{2}\right]+\Gamma_{c_{F}}\left[\left(\hat{c}_{F, t}^{i}\right)^{2}+\left(\hat{c}_{F, t}^{j}\right)^{2}\right]+ \\
& \Gamma_{l}\left[\left(\hat{l}_{t}^{i}\right)^{2}+\left(\hat{l}_{t}^{j}\right)^{2}\right]+\Gamma_{\Delta}\left[\hat{\Delta}_{t}^{i}+\hat{\Delta}_{t}^{j}\right]+ \\
& 2 \Gamma_{3}^{i i}\left\{\hat{\theta}_{t}^{i i}\left[\hat{e}_{t}^{i i} \Gamma_{4}-(1-\rho) \hat{e}_{t-1}^{i i}\right]+\hat{\theta}_{t}^{j j}\left[\hat{e}_{t}^{j j} \Gamma_{4}-(1-\rho) \hat{e}_{t-1}^{j j}\right]\right\}+ \\
& 2 \Gamma_{3}^{i j}\left\{\hat{\theta}_{t}^{i j}\left[\hat{e}_{t}^{i j} \Gamma_{4}-(1-\rho) \hat{e}_{t-1}^{i j}\right]+\hat{\theta}_{t}^{j i}\left[\hat{e}_{t}^{j i} \Gamma_{4}-(1-\rho) \hat{e}_{t-1}^{j i}\right]\right\}+ \\
& \left.2 \Gamma_{e^{i i} e^{j i}}\left[\hat{e}_{t}^{i i} \hat{e}_{t}^{j i}+\hat{e}_{t}^{j j} \hat{e}_{t}^{i j}\right]\right\} \text {, }
\end{aligned}
$$


where, at the symmetric steady state,

$$
\begin{aligned}
& \Gamma_{1}^{i i} \equiv\left(1-\alpha_{1}\right) \frac{E}{C}\left[\left[1-\alpha\left(1-\alpha_{1}\right)\right] \frac{1-\alpha}{E^{\alpha}}-\frac{\kappa}{q}-\frac{1-b}{b} \frac{C}{l}\left(1+\zeta \frac{1-f}{f}\right)\left(1-\frac{1}{\nu_{1}} \frac{f+\zeta(1-f)}{f+\zeta \rho(1-f)}\right)\right] \\
& \Gamma_{1}^{i j} \equiv \alpha_{1} \frac{E}{C}\left[\left[1-\alpha \alpha_{1}\right] \frac{1-\alpha}{E^{\alpha}}-\frac{\kappa}{q}-\frac{1-b}{b} \frac{C}{l}\left(1+\zeta \frac{1-f}{f}\right)\left(1-\frac{1}{\nu_{1}} \frac{f+\zeta(1-f)}{f+\zeta \rho(1-f)}\right)\right] \\
& \Gamma_{2}^{i i} \equiv\left(1-\alpha_{1}\right) \frac{E}{C}(1-\rho)\left[\frac{\kappa}{q}+\frac{1-b}{b} \frac{C}{l} \zeta \frac{1-f}{f}\left(1+\frac{1}{\nu_{1}} \frac{\zeta(1-\rho)(1-f)}{f+\zeta \rho(1-f)}\right)\right] \\
& \Gamma_{2}^{i j} \equiv \alpha_{1} \frac{E}{C}(1-\rho)\left[\frac{\kappa}{q}+\frac{1-b}{b} \frac{C}{l} \zeta \frac{1-f}{f}\left(1+\frac{1}{\nu_{1}} \frac{\zeta(1-\rho)(1-f)}{f+\zeta \rho(1-f)}\right)\right] \\
& \Gamma_{\theta}^{i i} \equiv-\left(1-\alpha_{1}\right) \frac{E}{C} \frac{\kappa}{q}(1-\gamma) \rho\left[1-\frac{1}{\nu_{1}} \frac{\gamma \zeta \rho}{f+\zeta \rho(1-f)}\right] \\
& \Gamma_{\theta}^{i j} \equiv-\alpha_{1} \frac{E}{C} \frac{\kappa}{q}(1-\gamma) \rho\left[1-\frac{1}{\nu_{1}} \frac{\gamma \zeta \rho}{f+\zeta \rho(1-f)}\right] \\
& \Gamma_{c} \equiv-\left(\frac{\nu_{2}-1}{\nu_{2}}\right) \quad ; \quad \Gamma_{c_{H}} \equiv-\frac{1-\alpha_{2}}{\nu_{2}} ; \quad \Gamma_{c_{F}} \equiv-\frac{\alpha_{2}}{\nu_{2}} \\
& \Gamma_{l} \equiv-\frac{1-b}{b}\left(1+\frac{1}{\nu_{1}} \frac{l}{1-1}\right) \quad ; \quad \Gamma_{\Delta} \equiv-2 \frac{1-\alpha}{E^{\alpha}} \frac{E}{C} \\
& \Gamma_{3}^{i i} \equiv-\frac{1-\alpha_{1}}{\nu_{1}} \frac{E}{C} \frac{\kappa}{q} \frac{\rho \zeta(1-f)(1-\gamma)}{f+\zeta \rho(1-f)} \quad ; \quad \Gamma_{3}^{i j} \equiv-\frac{\alpha_{1}}{\nu_{1}} \frac{E}{C} \frac{\kappa}{q} \frac{\rho \zeta(1-f)(1-\gamma)}{f+\zeta \rho(1-f)} \\
& \Gamma_{4} \equiv \frac{f+\zeta(1-f)}{\zeta(1-f)} ; \quad \Gamma_{e^{i i} e^{j i}} \equiv-\left[\alpha_{1}\left(1-\alpha_{1}\right) \frac{\alpha(1-\alpha)}{E^{\alpha}} \frac{E}{C}\right] \\
& \Gamma_{e}^{i i} \equiv \Gamma_{1}^{i i}+\beta \Gamma_{2}^{i i}=-\left(1-\alpha_{1}\right) \frac{E}{C}\left\{\frac{\alpha(1-\alpha)\left(1-\alpha_{1}\right)}{E^{\alpha}}-\frac{1-b}{b} \frac{C}{l} \frac{1}{\nu_{1}} \frac{f}{f+\zeta \rho(1-f)}\right. \\
& \left.\left[\left(1+\zeta \frac{1-f}{f}\right)^{2}-\beta(1-\rho)^{2}\left(\frac{1-f}{f} \zeta\right)^{2}\right]\right\} \\
& \Gamma_{e}^{i j} \equiv \Gamma_{1}^{i j}+\beta \Gamma_{2}^{i j}=-\alpha_{1} \frac{E}{C}\left\{\frac{\alpha(1-\alpha) \alpha_{1}}{E^{\alpha}}-\frac{1-b}{b} \frac{C}{l} \frac{1}{\nu_{1}} \frac{\alpha_{1}}{1-\alpha_{1}} \frac{f}{f+\zeta \rho(1-f)}\right. \\
& \left.\left[\left(1+\zeta \frac{1-f}{f}\right)^{2}-\beta(1-\rho)^{2}\left(\frac{1-f}{f} \zeta\right)^{2}\right]\right\} \text {. }
\end{aligned}
$$


Hence, the square terms appearing in the welfare function are

$$
\begin{aligned}
& \frac{1}{2} \sum_{t=0}^{\infty} \beta^{t}\left\{\Gamma_{e}^{i i}\left[\left(\hat{e}_{t}^{i i}\right)^{2}+\left(\hat{e}_{t}^{j j}\right)^{2}\right]+\Gamma_{e}^{i j}\left[\left(\hat{e}_{t}^{i j}\right)^{2}+\left(\hat{e}_{t}^{j i}\right)^{2}\right]\right. \\
& \Gamma_{\theta}^{i i}\left[\left(\hat{\theta}_{t}^{i i}\right)^{2}+\left(\hat{\theta}_{t}^{j j}\right)^{2}\right]+\Gamma_{\theta}^{i j}\left[\left(\hat{\theta}_{t}^{i j}\right)^{2}+\left(\hat{\theta}_{t}^{j i}\right)^{2}\right]+ \\
& \Gamma_{c}\left[\left(\hat{c}_{t}^{i}\right)^{2}+\left(\hat{c}_{t}^{j}\right)^{2}\right]+\Gamma_{c_{H}}\left[\left(\hat{c}_{H, t}^{i}\right)^{2}+\left(\hat{c}_{H, t}^{j}\right)^{2}\right]+\Gamma_{c_{F}}\left[\left(\hat{c}_{F, t}^{i}\right)^{2}+\left(\hat{c}_{F, t}^{j}\right)^{2}\right]+ \\
& \Gamma_{l}\left[\left(\hat{l}_{t}^{i}\right)^{2}+\left(\hat{l}_{t}^{j}\right)^{2}\right]+\Gamma_{\Delta}\left[\hat{\Delta}_{t}^{i}+\hat{\Delta}_{t}^{j}\right]+ \\
& 2 \Gamma_{3}^{i i}\left\{\hat{\theta}_{t}^{i i}\left[\hat{e}_{t}^{i i} \Gamma_{4}-(1-\rho) \hat{e}_{t-1}^{i i}\right]+\hat{\theta}_{t}^{j j}\left[\hat{e}_{t}^{j j} \Gamma_{4}-(1-\rho) \hat{e}_{t-1}^{j j}\right]\right\}+ \\
& 2 \Gamma_{3}^{i j}\left\{\hat{\theta}_{t}^{i j}\left[\hat{e}_{t}^{i j} \Gamma_{4}-(1-\rho) \hat{e}_{t-1}^{i j}\right]+\hat{\theta}_{t}^{j i}\left[\hat{e}_{t}^{j i} \Gamma_{4}-(1-\rho) \hat{e}_{t-1}^{j i}\right]\right\}+ \\
& \left.2 \Gamma_{e^{i i} e^{j i}}\left[\hat{e}_{t}^{i i} \hat{e}_{t}^{j i}+\hat{e}_{t}^{j j} \hat{e}_{t}^{i j}\right]\right\} .
\end{aligned}
$$

Appendix G.4. Cross-products with the shocks

Finally, we are left with the second-order terms, where the shocks are multiplied by endogenous variables.

$$
\sum_{t=0}^{\infty} \beta^{t} \Gamma_{a e}\left\{\hat{a}_{t}^{i}\left[\left(1-\alpha_{1}\right) \hat{e}_{t}^{i i}+\alpha_{1} \hat{e}_{t}^{j i}\right]+\hat{a}_{t}^{j}\left[\left(1-\alpha_{1}\right) \hat{e}_{t}^{j j}+\alpha_{1} \hat{e}_{t}^{i j}\right]\right\}+t . i . p .
$$

where

$$
\Gamma_{a e} \equiv\left[\frac{(1-\alpha)^{2}}{E^{\alpha}} \frac{E}{C}\right]
$$


Appendix G.5. Second-order approximation of the utility function

Collecting all quadratic terms yields

$$
\begin{aligned}
W & \simeq \mathbf{E}_{\mathbf{0}} \sum_{t=0}^{\infty} \beta^{t}\left[\left(\frac{U_{t}^{i}-U^{i}}{U_{c}^{i}}\right)+\left(\frac{U_{t}^{j}-U^{j}}{U_{c}^{j}}\right)\right] \\
& =\frac{1}{2} \mathbf{E}_{\mathbf{0}} \sum_{t=0}^{\infty} \beta^{t}\left\{\Gamma_{e}^{i i}\left[\left(\hat{e}_{t}^{i i}\right)^{2}+\left(\hat{e}_{t}^{j j}\right)^{2}\right]+\Gamma_{e}^{i j}\left[\left(\hat{e}_{t}^{i j}\right)^{2}+\left(\hat{e}_{t}^{j i}\right)^{2}\right]+\right. \\
& \Gamma_{\theta}^{i i}\left[\left(\hat{\theta}_{t}^{i i}\right)^{2}+\left(\hat{\theta}_{t}^{j j}\right)^{2}\right]+\Gamma_{\theta}^{i j}\left[\left(\hat{\theta}_{t}^{i j}\right)^{2}+\left(\hat{\theta}_{t}^{j i}\right)^{2}\right]+ \\
& \Gamma_{c}\left[\left(\hat{c}_{t}^{i}\right)^{2}+\left(\hat{c}_{t}^{j}\right)^{2}\right]+\Gamma_{c_{H}}\left[\left(\hat{c}_{H, t}^{i}\right)^{2}+\left(\hat{c}_{H, t}^{j}\right)^{2}\right]+\Gamma_{c_{F}}\left[\left(\hat{c}_{F, t}^{i}\right)^{2}+\left(\hat{c}_{F, t}^{j}\right)^{2}\right]+ \\
& \Gamma_{l}\left[\left(\hat{l}_{t}^{i}\right)^{2}+\left(\hat{l}_{t}^{j}\right)^{2}\right]+\Gamma_{\Delta}\left[\hat{\Delta}_{t}^{i}+\hat{\Delta}_{t}^{j}\right]+ \\
& 2 \Gamma_{3}^{i i}\left\{\hat{\theta}_{t}^{i i}\left[\hat{e}_{t}^{i i} \Gamma_{4}-(1-\rho) \hat{e}_{t-1}^{i i}\right]+\hat{\theta}_{t}^{j j}\left[\hat{e}_{t}^{j j} \Gamma_{4}-(1-\rho) \hat{e}_{t-1}^{j j}\right]\right\}+ \\
& 2 \Gamma_{3}^{i j}\left\{\hat{\theta}_{t}^{i j}\left[\hat{e}_{t}^{i j} \Gamma_{4}-(1-\rho) \hat{e}_{t-1}^{i j}\right]+\hat{\theta}_{t}^{j i}\left[\hat{e}_{t}^{j i} \Gamma_{4}-(1-\rho) \hat{e}_{t-1}^{j i}\right]\right\}+ \\
& 2 \Gamma_{e^{i i}}^{i i} e^{j i}\left[\hat{e}_{t}^{i i} \hat{e}_{t}^{j i}+\hat{e}_{t}^{j j} \hat{e}_{t}^{i j}\right]+ \\
& \left.2 \Gamma_{a e}\left\{\hat{a}_{t}^{i}\left[\left(1-\alpha_{1}\right) \hat{e}_{t}^{i i}+\alpha_{1} \hat{e}_{t}^{j i}\right]+\hat{a}_{t}^{j}\left[\left(1-\alpha_{1}\right) \hat{e}_{t}^{j j}+\alpha_{1} \hat{e}_{t}^{i j}\right]\right\}+t . i . p .\right\} .
\end{aligned}
$$

Appendix G.6. Welfare function

The second-order approximation of the utility function can be rewritten as a sum of squared deviations of endogenous variables from their Pareto efficient level. For a generic variable specific to region $i, X_{t}^{i}$, let us define $\tilde{x}_{t}^{i} \equiv \log X_{t}^{i}-\log X_{t}^{i \star}$, such that $\hat{x}_{t}^{i}=\tilde{x}_{t}^{i}+\hat{x}_{t}^{i \star}$. Such a variable represents the gap between the market solution and the efficient one. It is useful to define the deviation of the efficient allocation for the (efficient) steady state as $\hat{x}_{t}^{i \star} \equiv \log X_{t}^{i \star}-\log X^{i}$. When we use these definitions, (G.9) becomes 


$$
\begin{aligned}
& W \simeq \frac{1}{2} \mathbf{E}_{\mathbf{0}} \sum_{t=0}^{\infty} \beta^{t}\left\{\Gamma_{e}^{i i}\left[\left(\tilde{e}_{t}^{i i}\right)^{2}+\left(\tilde{e}_{t}^{j j}\right)^{2}\right]+\Gamma_{e}^{i j}\left[\left(\tilde{e}_{t}^{i j}\right)^{2}+\left(\tilde{e}_{t}^{j i}\right)^{2}\right]+\right. \\
& \Gamma_{\theta}^{i i}\left[\left(\tilde{\theta}_{t}^{i i}\right)^{2}+\left(\tilde{\theta}_{t}^{j j}\right)^{2}\right]+\Gamma_{\theta}^{i j}\left[\left(\tilde{\theta}_{t}^{i j}\right)^{2}+\left(\tilde{\theta}_{t}^{j i}\right)^{2}\right]+ \\
& \Gamma_{c}\left[\left(\tilde{c}_{t}^{i}\right)^{2}+\left(\tilde{c}_{t}^{j}\right)^{2}\right]+\Gamma_{c_{H}}\left[\left(\tilde{c}_{H, t}^{i}\right)^{2}+\left(\tilde{c}_{H, t}^{j}\right)^{2}\right]+\Gamma_{c_{F}}\left[\left(\tilde{c}_{F, t}^{i}\right)^{2}+\left(\tilde{c}_{F, t}^{j}\right)^{2}\right]+ \\
& \left.\Gamma_{l}\left[\left(\tilde{l}_{t}^{i}\right)^{2}+\left(\tilde{l}_{t}^{j}\right)^{2}\right]+\Gamma_{\Delta}\left[\tilde{\Delta}_{t}^{i}+\tilde{\Delta}_{t}^{j}\right]\right\}+ \\
& \mathbf{E}_{\mathbf{0}} \sum_{t=0}^{\infty} \beta^{t}\left\{\Gamma_{3}^{i i}\left\{\tilde{\theta}_{t}^{i i}\left[\tilde{e}_{t}^{i i} \Gamma_{4}-(1-\rho) \tilde{e}_{t-1}^{i i}\right]+\tilde{\theta}_{t}^{j j}\left[\tilde{e}_{t}^{j j} \Gamma_{4}-(1-\rho) \tilde{e}_{t-1}^{j j}\right]\right\}+\right. \\
& \Gamma_{3}^{i j}\left\{\tilde{\theta}_{t}^{i j}\left[\tilde{e}_{t}^{i j} \Gamma_{4}-(1-\rho) \tilde{e}_{t-1}^{i j}\right]+\tilde{\theta}_{t}^{j i}\left[\tilde{e}_{t}^{j i} \Gamma_{4}-(1-\rho) \tilde{e}_{t-1}^{j i}\right]\right\}+ \\
& \left.\Gamma_{e^{i i} e^{j i}}\left(\tilde{e}_{t}^{i i} \tilde{e}_{t}^{j i}+\tilde{e}_{t}^{j j} \tilde{e}_{t}^{i j}\right)\right\}+ \\
& \mathbf{E}_{\mathbf{0}} \sum_{t=0}^{\infty} \beta^{t}\left\{\Gamma_{e}^{i i}\left(\tilde{e}_{t}^{i i} \hat{e}_{t}^{i i \star}+\tilde{e}_{t}^{j j} \hat{e}_{t}^{j j \star}\right)+\Gamma_{e}^{i j}\left(\tilde{e}_{t}^{i j} \hat{e}_{t}^{i j \star}+\tilde{e}_{t}^{j i} \hat{e}_{t}^{j i \star}\right)+\right. \\
& \Gamma_{\theta}^{i i}\left(\tilde{\theta}_{t}^{i i} \hat{\theta}_{t}^{i i \star}+\tilde{\theta}_{t}^{j j} \hat{\theta}_{t}^{j j \star}\right)+\Gamma_{\theta}^{i j}\left(\tilde{\theta}_{t}^{i j} \hat{\theta}_{t}^{i j \star}+\tilde{\theta}_{t}^{j i} \hat{\theta}_{t}^{j i \star}\right)+ \\
& \Gamma_{c}\left(\tilde{c}_{t}^{i} \hat{c}_{t}^{i \star}+\tilde{c}_{t}^{j} \hat{c}_{t}^{j \star}\right)+\Gamma_{c_{H}}\left(\tilde{c}_{H, t}^{i} \hat{c}_{H, t}^{\star \star}+\tilde{c}_{H, t}^{j} \hat{c}_{H, t}^{j \star}\right)+\Gamma_{c_{F}}\left(\tilde{c}_{F, t}^{i} \hat{c}_{F, t}^{i \star}+\tilde{c}_{F, t}^{j} \hat{c}_{F, t}^{j \star}\right)+ \\
& \Gamma_{l}\left(\tilde{l}_{t}^{i} \hat{l}_{t}^{i \star}+\tilde{l}_{t}^{j} \hat{l}_{t}^{j \star}\right)+ \\
& \Gamma_{3}^{i i}\left[\Gamma_{4}\left(\tilde{\theta}_{t}^{i i} \hat{e}_{t}^{i i \star}+\tilde{e}_{t}^{i i} \hat{\theta}_{t}^{i i \star}+\tilde{\theta}_{t}^{j j} \hat{e}_{t}^{j j \star}+\tilde{e}_{t}^{j j} \hat{\theta}_{t}^{j j \star}\right)-(1-\rho)\left(\tilde{\theta}_{t}^{i i} \hat{e}_{t-1}^{i i \star}+\tilde{e}_{t-1}^{i i} \hat{\theta}_{t}^{i i \star}+\tilde{\theta}_{t}^{j j} \hat{e}_{t-1}^{j j \star}+\tilde{e}_{t-1}^{j j} \hat{\theta}_{t}^{j j \star}\right)\right]+ \\
& \Gamma_{3}^{i j}\left[\Gamma_{4}\left(\tilde{\theta}_{t}^{i j} \hat{e}_{t}^{i j \star}+\tilde{e}_{t}^{i j} \hat{\theta}_{t}^{i j \star}+\tilde{\theta}_{t}^{j i} \hat{e}_{t}^{j i \star}+\tilde{e}_{t}^{j i} \hat{\theta}_{t}^{j i \star}\right)-(1-\rho)\left(\tilde{\theta}_{t}^{i j} \hat{e}_{t-1}^{i j \star}+\tilde{e}_{t-1}^{i j} \hat{\theta}_{t}^{i j \star}+\tilde{\theta}_{t}^{j i} \hat{e}_{t-1}^{j i \star}+\tilde{e}_{t-1}^{j i} \hat{\theta}_{t}^{j i \star}\right)\right]+ \\
& \Gamma_{e^{i i} e^{j i}}\left[\tilde{e}_{t}^{i i} \hat{e}_{t}^{j i \star}+\tilde{e}_{t}^{j i} \hat{e}_{t}^{i i \star}+\tilde{e}_{t}^{j j} \hat{e}_{t}^{i j \star}+\tilde{e}_{t}^{i j} \hat{e}_{t}^{j j \star}\right]+ \\
& \left.\Gamma_{a e}\left\{\hat{a}_{t}^{i}\left[\left(1-\alpha_{1}\right) \tilde{e}_{t}^{i i}+\alpha_{1} \tilde{e}_{t}^{j i}\right]+\hat{a}_{t}^{j}\left[\left(1-\alpha_{1}\right) \tilde{e}_{t}^{j j}+\alpha_{1} \tilde{e}_{t}^{i j}\right]\right\}\right\}+ \text { t.i.p. }
\end{aligned}
$$


Define

$$
\begin{aligned}
& A_{0} \equiv \mathbf{E}_{\mathbf{0}} \sum_{t=0}^{\infty} \beta^{t}\left\{\Gamma_{e}^{i i}\left(\tilde{e}_{t}^{i i} \hat{e}_{t}^{i i \star}+\tilde{e}_{t}^{j j} \hat{e}_{t}^{j j \star}\right)+\Gamma_{e}^{i j}\left(\tilde{e}_{t}^{i j} \hat{e}_{t}^{i j \star}+\tilde{e}_{t}^{j i} \hat{e}_{t}^{j i \star}\right)+\right. \\
& \Gamma_{\theta}^{i i}\left(\tilde{\theta}_{t}^{i i} \hat{\theta}_{t}^{i i \star}+\tilde{\theta}_{t}^{j j} \hat{\theta}_{t}^{j j \star}\right)+\Gamma_{\theta}^{i j}\left(\tilde{\theta}_{t}^{i j} \hat{\theta}_{t}^{i j \star}+\tilde{\theta}_{t}^{j i} \hat{\theta}_{t}^{j i \star}\right)+ \\
& \Gamma_{c}\left(\tilde{c}_{t}^{i} \hat{c}_{t}^{i \star}+\tilde{c}_{t}^{j} \hat{c}_{t}^{j \star}\right)+\Gamma_{c_{H}}\left(\tilde{c}_{H, t}^{i} \hat{c}_{H, t}^{i \star}+\tilde{c}_{H, t}^{j} \hat{c}_{H, t}^{j \star}\right)+\Gamma_{c_{F}}\left(\tilde{c}_{F, t}^{i} \hat{c}_{F, t}^{i \star}+\tilde{c}_{F, t}^{j} \hat{c}_{F, t}^{j \star}\right)+ \\
& \Gamma_{l}\left(\tilde{l}_{t}^{i} \hat{l}_{t}^{i \star}+\tilde{l}_{t}^{j} \hat{l}_{t}^{j \star}\right)+ \\
& \Gamma_{3}^{i i}\left[\Gamma_{4}\left(\tilde{\theta}_{t}^{i i} \hat{e}_{t}^{i i \star}+\tilde{e}_{t}^{i i} \hat{\theta}_{t}^{i i \star}+\tilde{\theta}_{t}^{j j} \hat{e}_{t}^{j j \star}+\tilde{e}_{t}^{j j} \hat{\theta}_{t}^{j j \star}\right)-(1-\rho)\left(\tilde{\theta}_{t}^{i i} \hat{e}_{t-1}^{i i \star}+\tilde{e}_{t-1}^{i i} \hat{\theta}_{t}^{i i \star}+\tilde{\theta}_{t}^{j j} \hat{e}_{t-1}^{j j \star}+\tilde{e}_{t-1}^{j j} \hat{\theta}_{t}^{j j \star}\right)\right]+ \\
& \Gamma_{3}^{i j}\left[\Gamma_{4}\left(\tilde{\theta}_{t}^{i j} \hat{e}_{t}^{i j \star}+\tilde{e}_{t}^{i j} \hat{\theta}_{t}^{i j \star}+\tilde{\theta}_{t}^{j i} \hat{e}_{t}^{j i \star}+\tilde{e}_{t}^{j i} \hat{\theta}_{t}^{j i \star}\right)-(1-\rho)\left(\tilde{\theta}_{t}^{i j} \hat{e}_{t-1}^{i j \star}+\tilde{e}_{t-1}^{i j} \hat{\theta}_{t}^{i j \star}+\tilde{\theta}_{t}^{j i} \hat{e}_{t-1}^{j i \star}+\tilde{e}_{t-1}^{j i} \hat{\theta}_{t}^{j i \star}\right)\right]+ \\
& \Gamma_{e^{i i}} e^{j i}\left[\tilde{e}_{t}^{i i} \hat{e}_{t}^{j i \star}+\tilde{e}_{t}^{j i} \hat{e}_{t}^{i i \star}+\tilde{e}_{t}^{j j} \hat{e}_{t}^{i j \star}+\tilde{e}_{t}^{i j} \hat{e}_{t}^{j j \star}\right]+ \\
& \left.\Gamma_{a e}\left\{\hat{a}_{t}^{i}\left[\left(1-\alpha_{1}\right) \tilde{e}_{t}^{i i}+\alpha_{1} \tilde{e}_{t}^{j i}\right]+\hat{a}_{t}^{j}\left[\left(1-\alpha_{1}\right) \tilde{e}_{t}^{j j}+\alpha_{1} \tilde{e}_{t}^{i j}\right]\right\}\right\}+t . i . p .
\end{aligned}
$$

One can show that around the efficient symmetric steady state, $A_{0}=0$. First, using the feasibility constraint (G.6) up to first order around the symmetric steady state yields

$$
\begin{aligned}
& \mathbf{E}_{\mathbf{0}} \sum_{t=0}^{\infty} \beta^{t} \Gamma_{c} \tilde{c}_{t}^{i} \hat{c}_{t}^{i \star}= \\
& \mathbf{E}_{\mathbf{0}} \sum_{t=0}^{\infty} \beta^{t}\left\{-\alpha_{2} \frac{\nu_{2}-1}{\nu_{2}}\left(\tilde{c}_{F, t}^{i} \hat{c}_{t}^{i \star}-\tilde{c}_{F, t}^{j} \hat{c}_{t}^{i \star}\right)-\frac{E}{C} \frac{\nu_{2}-1}{\nu_{2}}\left[\frac{1-\alpha}{E^{\alpha}}-\frac{\kappa}{q}\right]\left[\left(1-\alpha_{1}\right) \tilde{e}_{t}^{i i} \hat{c}_{t}^{i \star}+\alpha_{1} \tilde{e}_{t}^{j i} \hat{c}_{t}^{i \star}\right]+\right. \\
& \left.\frac{E}{C} \frac{\nu_{2}-1}{\nu_{2}} \frac{\kappa}{q}(1-\gamma) \rho\left[\left(1-\alpha_{1}\right) \tilde{\theta}_{t}^{i i} \hat{c}_{t}^{i \star}+\alpha_{1} \tilde{\theta}_{t}^{j i} \hat{c}_{t}^{i \star}\right]-\frac{E}{C} \frac{\nu_{2}-1}{\nu_{2}} \frac{\kappa}{q} \beta(1-\rho)\left[\left(1-\alpha_{1}\right) \tilde{e}_{t}^{i i} \hat{c}_{t+1}^{i \star}+\alpha_{1} \tilde{e}_{t}^{j i} \hat{c}_{t+1}^{i \star}\right]\right\} \\
& +t . i . p .
\end{aligned}
$$

An equivalent expression can be derived for region $j$. The second feasibility constraint (G.7), up to the first order around the symmetric steady state, implies

$$
\begin{aligned}
& \mathbf{E}_{\mathbf{0}} \sum_{t=0}^{\infty} \beta^{t} \Gamma_{l} \tilde{l}_{t}^{i} \hat{l}_{t}^{i \star}= \\
& \mathbf{E}_{\mathbf{0}} \sum_{t=0}^{\infty} \beta^{t}\left\{\frac{1-b}{b} \frac{E}{l}\left(1+\zeta \frac{1-f}{f}\right)\left(1+\frac{1}{\nu_{1}} \frac{l}{1-l}\right)\left[\left(1-\alpha_{1}\right) \tilde{e}_{t}^{i i} \hat{l}_{t}^{i \star}+\alpha_{1} \tilde{e}_{t}^{i j} \hat{l}_{t}^{i \star}\right]-\right. \\
& \frac{1-b}{b} \frac{E}{l} \frac{\rho \zeta \gamma}{f}\left(1+\frac{1}{\nu_{1}} \frac{l}{1-l}\right)\left[\left(1-\alpha_{1}\right) \tilde{\theta}_{t}^{i i} \hat{l}_{t}^{i \star}+\alpha_{1} \tilde{\theta}_{t}^{i j} \hat{l}_{t}^{i \star}\right]- \\
& \left.\frac{1-b}{b} \frac{E}{l} \beta(1-\rho) \zeta \frac{1-f}{f}\left(1+\frac{1}{\nu_{1}} \frac{l}{1-l}\right)\left[\left(1-\alpha_{1}\right) \tilde{e}_{t}^{i i} \hat{l}_{t+1}^{i \star}+\alpha_{1} \tilde{e}_{t}^{i j} \hat{l}_{t+1}^{i \star}\right]\right\}+t . i . p .
\end{aligned}
$$


An equivalent expression can be derived for region $j$. We now collect all terms for $\tilde{\theta}_{t}^{i i}$ :

$$
\begin{aligned}
\tilde{\theta}_{t}^{i i}\left\{\Gamma_{\theta}^{i i} \hat{\theta}_{t}^{i i \star}+\frac{E}{C} \frac{\nu_{2}-1}{\nu_{2}} \frac{\kappa}{q}(1-\gamma) \rho\left(1-\alpha_{1}\right) \hat{c}_{t}^{i \star}-\frac{1-b}{b} \frac{E}{l} \frac{\rho \zeta \gamma}{f}\left(1+\frac{1}{\nu_{1}} \frac{l}{1-l}\right)\left(1-\alpha_{1}\right) \hat{l}_{t}^{i \star}+\right. \\
\left.\Gamma_{3}^{i i} \Gamma_{4} \hat{e}_{t}^{i i \star}-\Gamma_{3}^{i i}(1-\rho) \hat{e}_{t-1}^{i i \star}\right\} .
\end{aligned}
$$

From the efficiency condition with respect to $\theta_{t}^{i i}$, up to the first order around the efficient steady state, we have

$$
\begin{aligned}
-\left(1+\frac{1}{\nu_{1}} \frac{l}{1-l}\right) \hat{l}_{t}^{i \star}= & {\left[1-\frac{1}{\nu_{1}} \frac{\zeta \rho \gamma}{f} \frac{f}{f+\zeta \rho(1-f)}\right] \hat{\theta}_{t}^{i i \star}-\frac{\nu_{2}-1}{\nu_{2}} \hat{c}_{t}^{i \star}-\frac{1}{\nu_{2}} \hat{c}_{H, t}^{i \star}+} \\
& \frac{1}{\nu_{1}} \frac{f}{f+\zeta \rho(1-f)}\left[\left(1+\zeta \frac{1-f}{f}\right) \hat{e}_{t}^{i i \star}-\zeta(1-\rho) \frac{1-f}{f} \hat{e}_{t-1}^{i i \star}\right],
\end{aligned}
$$

such that

$$
\begin{aligned}
& -\frac{1-b}{b} \frac{E}{l} \frac{\rho \zeta \gamma}{f}\left(1-\alpha_{1}\right)\left(1+\frac{1}{\nu_{1}} \frac{l}{1-l}\right) \hat{l}_{t}^{i \star}=\frac{1-b}{b} \frac{E}{l} \frac{\rho \zeta \gamma}{f}\left(1-\alpha_{1}\right)\left[1-\frac{1}{\nu_{1}} \frac{\zeta \rho \gamma}{f} \frac{f}{f+\zeta \rho(1-f)}\right] \hat{\theta}_{t}^{i i \star}- \\
& \frac{1-b}{b} \frac{E}{l} \frac{\rho \zeta \gamma}{f}\left(1-\alpha_{1}\right) \frac{\nu_{2}-1}{\nu_{2}} \hat{c}_{t}^{i \star}-\frac{1-b}{b} \frac{E}{l} \frac{\rho \zeta \gamma}{f}\left(1-\alpha_{1}\right) \frac{1}{\nu_{2}} \hat{c}_{H, t}^{i \star}+ \\
& \frac{1-b}{b} \frac{E}{l} \frac{\rho \zeta \gamma}{f}\left(1-\alpha_{1}\right) \frac{1}{\nu_{1}} \frac{f}{f+\zeta \rho(1-f)}\left[\left(1+\zeta \frac{1-f}{f}\right) \hat{e}_{t}^{i i \star}-\zeta(1-\rho) \frac{1-f}{f} \hat{e}_{t-1}^{i i \star}\right] .
\end{aligned}
$$

The coefficient on $\hat{\theta}_{t}^{i i \star}$ appearing in the collection of terms for $\tilde{\theta}_{t}^{i i}$ then states as

$$
\begin{aligned}
& \Gamma_{\theta}^{i i}+\frac{1-b}{b} \frac{E}{l} \frac{\rho \zeta \gamma}{f}\left(1-\alpha_{1}\right)\left[1-\frac{1}{\nu_{1}} \frac{\zeta \rho \gamma}{f} \frac{f}{f+\zeta \rho(1-f)}\right]= \\
& -\left(1-\alpha_{1}\right) \frac{E}{C} \frac{\kappa}{q} \rho(1-\gamma)\left[1-\frac{1}{\nu_{1}} \frac{\zeta \rho \gamma}{f+\zeta \rho(1-f)}\right]+\left(1-\alpha_{1}\right) \frac{E}{C} \frac{\kappa}{q} \rho(1-\gamma)\left[1-\frac{1}{\nu_{1}} \frac{\zeta \rho \gamma}{f+\zeta \rho(1-f)}\right]=0
\end{aligned}
$$

where the last equality makes use of the steady-state equation (E.1). Similarly, the coefficient on $\hat{e}_{t}^{i i \star}$ reads as

$$
\left(1+\zeta \frac{1-f}{f}\right) \frac{1-\alpha_{1}}{\nu_{1}} \frac{\rho \zeta \gamma}{f+\zeta \rho(1-f)} \frac{1-b}{b} \frac{E}{l}-\left(1+\zeta \frac{1-f}{f}\right) \frac{1-\alpha_{1}}{\nu_{1}} \frac{\rho \zeta \gamma}{f+\zeta \rho(1-f)} \frac{1-b}{b} \frac{E}{l}=0 .
$$

Finally, the coefficient on $\hat{e}_{t-1}^{i i \star}$ reads as

$$
\frac{1-\alpha_{1}}{\nu_{1}} \frac{E}{C} \frac{\kappa}{q} \frac{\rho \zeta(1-f)(1-\gamma)(1-\rho)}{f+\zeta \rho(1-f)}-\frac{1-\alpha_{1}}{\nu_{1}} \frac{E}{C} \frac{\kappa}{q} \frac{\rho \zeta(1-f)(1-\gamma)(1-\rho)}{f+\zeta \rho(1-f)}=0,
$$

so that expression (G.10) reduces to

$$
\tilde{\theta}_{t}^{i i}\left\{-\frac{1-b}{b} \frac{E}{l} \frac{\rho \zeta \gamma}{f} \frac{1-\alpha_{1}}{\nu_{2}} \hat{c}_{H, t}^{i \star}\right\}
$$


Equivalently, collecting terms for $\tilde{\theta}_{t}^{i j}$ yields

$$
\tilde{\theta}_{t}^{i j}\left\{\frac{\nu_{2}-1}{\nu_{2}} \frac{E}{C} \frac{\kappa}{q}(1-\gamma) \rho \alpha_{1}\left(\hat{c}_{t}^{j \star}-\hat{c}_{t}^{i \star}\right)-\frac{\alpha_{1}}{\nu_{2}} \frac{1-b}{b} \frac{E}{l} \frac{\rho \zeta \gamma}{f} \hat{c}_{F, t}^{i \star}\right\} .
$$

We now collect all terms for $\tilde{e}_{t}^{i i}$ :

$$
\begin{aligned}
\tilde{e}_{t}^{i i}\{ & \Gamma_{e}^{i i} \hat{e}_{t}^{i i \star}+\Gamma_{3}^{i i} \Gamma_{4} \hat{\theta}_{t}^{i i \star}+\Gamma_{e^{i i} e^{j i}} \hat{e}_{t}^{j i \star}+\Gamma_{a e}\left(1-\alpha_{1}\right) \hat{a}_{t}^{i}-\frac{E}{C} \frac{\nu_{2}-1}{\nu_{2}}\left[\frac{1-\alpha}{E^{\alpha}}-\frac{\kappa}{q}\right]\left(1-\alpha_{1}\right) \hat{c}_{t}^{i \star}- \\
& \frac{E}{C} \frac{\nu_{2}-1}{\nu_{2}} \frac{\kappa}{q} \beta(1-\rho)\left(1-\alpha_{1}\right) \hat{c}_{t+1}^{i \star}+\frac{1-b}{b} \frac{E}{l}\left(1+\zeta \frac{1-f}{f}\right)\left(1+\frac{1}{\nu_{1}} \frac{l}{1-l}\right)\left(1-\alpha_{1}\right) \hat{l}_{t}^{i \star}- \\
& \left.\frac{1-b}{b} \frac{E}{l} \beta(1-\rho) \zeta \frac{1-f}{f}\left(1+\frac{1}{\nu_{1}} \frac{l}{1-l}\right)\left(1-\alpha_{1}\right) \hat{l}_{t+1}^{i \star}\right\} .
\end{aligned}
$$

From the efficiency condition with respect to $E_{t}^{i i}$, approximated up to the first order around the efficient steady state, we have

$$
\begin{aligned}
& \frac{\Gamma_{e}^{i i}}{\left(1-\alpha_{1}\right) \frac{E}{C}} \hat{e}_{t}^{i i \star}+\frac{(1-\alpha)^{2}}{E^{\alpha}} \hat{a}_{t}^{i}-\beta(1-\rho) \frac{\kappa}{q} \frac{\nu_{2}-1}{\nu_{2}} \hat{c}_{t+1}^{i \star}+\frac{1-b}{b} \frac{C}{l}\left(1+\zeta \frac{1-f}{f}\right)\left(1+\frac{1}{\nu_{1}} \frac{l}{1-l}\right) \hat{l}_{t}^{i \star}- \\
& \beta(1-\rho) \frac{1-b}{b} \frac{C}{l} \zeta \frac{1-f}{f}\left(1+\frac{1}{\nu_{1}} \frac{l}{1-l}\right) \hat{l}_{t+1}^{i \star}+\frac{\Gamma_{3}^{i i} \Gamma_{4}}{\left(1-\alpha_{1}\right) \frac{E}{C}} \hat{\theta}_{t}^{i i \star}+\frac{\Gamma_{e^{i i}} e^{j i}}{E^{\alpha}} \hat{e}_{t}^{j i \star}= \\
& \left\{\frac{1-b}{b} \frac{C}{l}\left(1+\zeta \frac{1-f}{f}\right)-\frac{1}{\nu_{2}}\left(\frac{1-\alpha}{E^{\alpha}}-\frac{\kappa}{q}\right)-\beta(1-\rho)\left(\frac{\kappa}{q}+\frac{1-b}{b} \frac{C}{l} \zeta \frac{1-f}{f}\right)\right\} \hat{c}_{t}^{i \star}+ \\
& \frac{1}{\nu_{2}}\left(\frac{1-\alpha}{E^{\alpha}}-\frac{\kappa}{q}\right) \hat{c}_{H, t}^{i \star}+\frac{1}{\nu_{2}} \beta(1-\rho) \frac{\kappa}{q} \hat{c}_{H, t+1}^{i \star}-\beta(1-\rho) \frac{\kappa}{q}(1-\gamma) \frac{1}{\nu_{1}} \frac{\zeta \rho(1-f)}{f+\zeta \rho(1-f)} \hat{\theta}_{t+1}^{i i \star}+ \\
& \frac{\kappa}{q} \frac{f-\gamma}{\gamma}\left(1+\zeta \frac{1-f}{f}\right) \frac{1}{\nu_{1}} \frac{f+\zeta \rho(1-f)}{f+\rho}(1-\rho)(1-f) \hat{e}_{t-1}^{i i \star}+ \\
& \beta(1-\rho)(1-f) \frac{\kappa}{q} \frac{1-\gamma}{\gamma} \frac{1}{\nu_{1}} \frac{f+\zeta(1-f)}{f+\zeta \rho(1-f)} \hat{e}_{t+1}^{i i \star},
\end{aligned}
$$

so that expression (G.13) can be rewritten as follows:

$$
\begin{aligned}
\tilde{e}_{t}^{i i}\left(1-\alpha_{1}\right) \frac{E}{C}\{ & {\left[\frac{\kappa}{q}(1-\beta(1-\rho))-\frac{1-\alpha}{E^{\alpha}}+\frac{1-b}{b} \frac{C}{l}\left(1+\zeta \frac{1-f}{f}(1-\beta(1-\rho))\right)\right] \hat{c}_{t}^{i \star}+} \\
& \frac{1}{\nu_{2}}\left(\frac{1-\alpha}{E^{\alpha}}-\frac{\kappa}{q}\right) \hat{c}_{H, t}^{i \star}+\frac{1}{\nu_{2}} \beta(1-\rho) \frac{\kappa}{q} \hat{c}_{H, t+1}^{i \star}- \\
& \beta(1-\rho) \frac{\kappa}{q}(1-\gamma) \frac{1}{\nu_{1}} \frac{\zeta \rho(1-f)}{f+\zeta \rho(1-f)} \hat{\theta}_{t+1}^{i i \star}+ \\
& \frac{\kappa}{q} \frac{1-\gamma}{\gamma}\left(1+\zeta \frac{1-f}{f}\right) \frac{1}{\nu_{1}} \frac{f}{f+\zeta \rho(1-f)}(1-\rho)(1-f) \hat{e}_{t-1}^{i i \star}+ \\
& \left.\beta(1-\rho) \frac{\kappa}{q} \frac{1-\gamma}{\gamma}(1-f) \frac{1}{\nu_{1}} \frac{f+\zeta(1-f)}{f+\zeta \rho(1-f)} \hat{e}_{t+1}^{i i \star}\right\} .
\end{aligned}
$$


The coefficient on $\hat{c}_{t}^{i \star}$ is equal to zero according to steady-state condition (E.2). Expression (G.14) thus reduces to

$$
\begin{aligned}
\tilde{e}_{t}^{i i}\left(1-\alpha_{1}\right) \frac{E}{C}\{ & \frac{1}{\nu_{2}}\left(\frac{1-\alpha}{E^{\alpha}}-\frac{\kappa}{q}\right) \hat{c}_{H, t}^{i \star}+\frac{1}{\nu_{2}} \beta(1-\rho) \frac{\kappa}{q} \hat{c}_{H, t+1}^{i \star}- \\
& \beta(1-\rho) \frac{\kappa}{q}(1-\gamma) \frac{1}{\nu_{1}} \frac{\zeta \rho(1-f)}{f+\zeta \rho(1-f)} \hat{\theta}_{t+1}^{i i \star}+ \\
& \frac{\kappa}{q} \frac{1-\gamma}{\gamma}\left(1+\zeta \frac{1-f}{f}\right) \frac{1}{\nu_{1}} \frac{f}{f+\zeta \rho(1-f)}(1-\rho)(1-f) \hat{e}_{t-1}^{i i \star}+ \\
& \left.\beta(1-\rho) \frac{\kappa}{q} \frac{1-\gamma}{\gamma}(1-f) \frac{1}{\nu_{1}} \frac{f+\zeta(1-f)}{f+\zeta \rho(1-f)} \hat{e}_{t+1}^{i i \star}\right\} .
\end{aligned}
$$

Equivalently, collecting terms for $\tilde{e}_{t}^{i j}$ yields

$$
\begin{aligned}
& \tilde{e}_{t}^{i j} \alpha_{1} \frac{E}{C}\left\{\frac{1}{\nu_{2}}\left(\frac{1-\alpha}{E^{\alpha}}-\frac{\kappa}{q}\right) \hat{c}_{F, t}^{i \star}+\frac{1}{\nu_{2}} \beta(1-\rho) \frac{\kappa}{q} \hat{c}_{F, t+1}^{i i}-\right. \\
& \beta(1-\rho) \frac{\kappa}{q}(1-\gamma) \frac{1}{\nu_{1}} \frac{\zeta \rho(1-f)}{f+\zeta \rho(1-f)} \hat{\theta}_{t+1}^{i j \star}+ \\
& \frac{\kappa}{q} \frac{1-\gamma}{\gamma}\left(1+\zeta \frac{1-f}{f}\right) \frac{1}{\nu_{1}} \frac{\alpha_{1}}{1-\alpha_{1}} \frac{f}{f+\zeta \rho(1-f)}(1-\rho)(1-f) \hat{e}_{t-1}^{i j \star}+ \\
& \beta(1-\rho) \frac{\kappa}{q} \frac{1-\gamma}{\gamma}(1-f) \frac{1}{\nu_{1}} \frac{f+\zeta(1-f)}{f+\zeta \rho(1-f)} \hat{e}_{t+1}^{i j \star}+ \\
&\left.\beta(1-\rho) \frac{\kappa}{q} \frac{\nu_{2}-1}{\nu_{2}}\left(\hat{c}_{t+1}^{i \star}-\hat{c}_{t+1}^{j \star}\right)+\frac{\nu_{2}-1}{\nu_{2}}\left(\frac{1-\alpha}{E^{\alpha}}-\frac{\kappa}{q}\right)\left(\hat{c}_{t}^{i \star}-\hat{c}_{t}^{j \star}\right)\right\},
\end{aligned}
$$


Hence, we can rewrite the initial expression for $A_{0}$ as follows:

$$
\begin{aligned}
& A_{0} \equiv \mathbf{E}_{\mathbf{0}} \sum_{t=0}^{\infty} \beta^{t}\left\{\tilde{\theta}_{t}^{i i}\left[-\frac{1-b}{b} \frac{E}{l} \frac{\rho \zeta \gamma}{f} \frac{1-\alpha_{1}}{\nu_{2}} \hat{c}_{H, t}^{i \star}\right]+\tilde{\theta}_{t}^{j j}\left[-\frac{1-b}{b} \frac{E}{l} \frac{\rho \zeta \gamma}{f} \frac{1-\alpha_{1}}{\nu_{2}} \hat{c}_{H, t}^{j \star}\right]+\right. \\
& \tilde{\theta}_{t}^{i j}\left[-\frac{E}{C} \rho(1-\gamma) \frac{\kappa}{q} \frac{\alpha_{1}}{\nu_{2}} \hat{c}_{H, t}^{j \star}\right]+\tilde{\theta}_{t}^{j i}\left[-\frac{E}{C} \rho(1-\gamma) \frac{\kappa}{q} \frac{\alpha_{1}}{\nu_{2}} \hat{c}_{H, t}^{i \star}\right]+ \\
& \tilde{e}_{t}^{i i}\left(1-\alpha_{1}\right) \frac{E}{C}\left[\frac{1}{\nu_{2}}\left(\frac{1-\alpha}{E^{\alpha}}-\frac{\kappa}{q}\right) \hat{c}_{H, t}^{i \star}+\frac{1}{\nu_{2}} \beta(1-\rho) \frac{\kappa}{q} \hat{c}_{H, t+1}^{i \star}\right]- \\
& \tilde{e}_{t}^{i i}\left(1-\alpha_{1}\right) \frac{E}{C}\left[\beta(1-\rho) \frac{\kappa}{q}(1-\gamma) \frac{1}{\nu_{1}} \frac{\zeta \rho(1-f)}{f+\zeta \rho(1-f)} \hat{\theta}_{t+1}^{i i \star}\right]+ \\
& \tilde{e}_{t}^{i i}\left(1-\alpha_{1}\right) \frac{E}{C}\left[\frac{\kappa}{q} \frac{1-\gamma}{\gamma}\left(1+\zeta \frac{1-f}{f}\right) \frac{1}{\nu_{1}} \frac{f}{f+\zeta \rho(1-f)}(1-\rho)(1-f) \hat{e}_{t-1}^{i i \star}\right]+ \\
& \tilde{e}_{t}^{i i}\left(1-\alpha_{1}\right) \frac{E}{C}\left[\beta(1-\rho) \frac{\kappa}{q} \frac{1-\gamma}{\gamma}(1-f) \frac{1}{\nu_{1}} \frac{f+\zeta(1-f)}{f+\zeta \rho(1-f)} \hat{e}_{t+1}^{i i \star}\right]+ \\
& \tilde{e}_{t}^{j j}\left(1-\alpha_{1}\right) \frac{E}{C}\left[\frac{1}{\nu_{2}}\left(\frac{1-\alpha}{E^{\alpha}}-\frac{\kappa}{q}\right) \hat{c}_{H, t}^{j \star}+\frac{1}{\nu_{2}} \beta(1-\rho) \frac{\kappa}{q} \hat{c}_{H, t+1}^{j \star}\right]- \\
& \tilde{e}_{t}^{j j}\left(1-\alpha_{1}\right) \frac{E}{C}\left[\beta(1-\rho) \frac{\kappa}{q}(1-\gamma) \frac{1}{\nu_{1}} \frac{\zeta \rho(1-f)}{f+\zeta \rho(1-f)} \hat{\theta}_{t+1}^{j j \star}\right]+ \\
& \tilde{e}_{t}^{j j}\left(1-\alpha_{1}\right) \frac{E}{C}\left[\frac{\kappa}{q} \frac{1-\gamma}{\gamma}\left(1+\zeta \frac{1-f}{f}\right) \frac{1}{\nu_{1}} \frac{f}{f+\zeta \rho(1-f)}(1-\rho)(1-f) \hat{e}_{t-1}^{j j \star}\right]+ \\
& \tilde{e}_{t}^{j j}\left(1-\alpha_{1}\right) \frac{E}{C}\left[\beta(1-\rho) \frac{\kappa}{q} \frac{1-\gamma}{\gamma}(1-f) \frac{1}{\nu_{1}} \frac{f+\zeta(1-f)}{f+\zeta \rho(1-f)} \hat{e}_{t+1}^{j j \star}\right]+ \\
& \tilde{e}_{t}^{i j} \alpha_{1} \frac{E}{C}\left[\frac{1}{\nu_{2}}\left(\frac{1-\alpha}{E^{\alpha}}-\frac{\kappa}{q}\right) \hat{c}_{H, t}^{j \star}+\frac{1}{\nu_{2}} \beta(1-\rho) \frac{\kappa}{q} \hat{c}_{H, t+1}^{j \star}\right]- \\
& \tilde{e}_{t}^{i j} \alpha_{1} \frac{E}{C}\left[\beta(1-\rho) \frac{\kappa}{q}(1-\gamma) \frac{1}{\nu_{1}} \frac{\zeta \rho(1-f)}{f+\zeta \rho(1-f)} \hat{\theta}_{t+1}^{i j \star}\right]+ \\
& \tilde{e}_{t}^{i j} \alpha_{1} \frac{E}{C}\left[\frac{\kappa}{q} \frac{1-\gamma}{\gamma}\left(1+\zeta \frac{1-f}{f}\right) \frac{1}{\nu_{1}} \frac{\alpha_{1}}{1-\alpha_{1}} \frac{f}{f+\zeta \rho(1-f)}(1-\rho)(1-f) \hat{e}_{t-1}^{i j \star}\right]+ \\
& \tilde{e}_{t}^{i j} \alpha_{1} \frac{E}{C}\left[\beta(1-\rho) \frac{\kappa}{q} \frac{1-\gamma}{\gamma}(1-f) \frac{1}{\nu_{1}} \frac{f+\zeta(1-f)}{f+\zeta \rho(1-f)} \hat{e}_{t+1}^{i j \star}\right]+ \\
& \tilde{e}_{t}^{j i} \alpha_{1} \frac{E}{C}\left[\frac{1}{\nu_{2}}\left(\frac{1-\alpha}{E^{\alpha}}-\frac{\kappa}{q}\right) \hat{c}_{H, t}^{i \star}+\frac{1}{\nu_{2}} \beta(1-\rho) \frac{\kappa}{q} \hat{c}_{H, t+1}^{i \star}\right]- \\
& \tilde{e}_{t}^{j i} \alpha_{1} \frac{E}{C}\left[\beta(1-\rho) \frac{\kappa}{q}(1-\gamma) \frac{1}{\nu_{1}} \frac{\zeta \rho(1-f)}{f+\zeta \rho(1-f)} \hat{\theta}_{t+1}^{j i \star}\right]+ \\
& \tilde{e}_{t}^{j i} \alpha_{1} \frac{E}{C}\left[\frac{\kappa}{q} \frac{1-\gamma}{\gamma}\left(1+\zeta \frac{1-f}{f}\right) \frac{1}{\nu_{1}} \frac{\alpha_{1}}{1-\alpha_{1}} \frac{f}{f+\zeta \rho(1-f)}(1-\rho)(1-f) \hat{e}_{t-1}^{j i \star}\right]+ \\
& \tilde{e}_{t}^{j i} \alpha_{1} \frac{E}{C}\left[\beta(1-\rho) \frac{\kappa}{q} \frac{1-\gamma}{\gamma}(1-f) \frac{1}{\nu_{1}} \frac{f+\zeta(1-f)}{f+\zeta \rho(1-f)} \hat{e}_{t+1}^{j i \star}\right]+ \\
& \Gamma_{c_{H}}\left(\tilde{c}_{H, t}^{i} \hat{c}_{H, t}^{i \star}+\tilde{c}_{H, t}^{j} \hat{c}_{H, t}^{j \star}\right)+\Gamma_{c_{F}}\left(\tilde{c}_{F, t}^{i} \hat{c}_{H, t}^{j \star}+\tilde{c}_{F, t}^{j} \hat{c}_{H, t}^{i \star}\right)- \\
& \left.\Gamma_{3}^{i i}(1-\rho)\left[\tilde{e}_{t-1}^{i i} \hat{\theta}_{t}^{i i \star}+\tilde{e}_{t-1}^{j j} \hat{\theta}_{t}^{j j \star}\right]-\Gamma_{3}^{i j}(1-\rho)\left[\tilde{e}_{t-1}^{i j} \hat{\theta}_{t}^{i j \star}+\tilde{e}_{t-1}^{j i} \hat{\theta}_{t}^{j i \star}\right]\right\} \text {. }
\end{aligned}
$$


Finally, collecting terms in $\hat{c}_{H, t}^{i \star}, \hat{c}_{H, t}^{j \star}, \hat{\theta}_{t}^{i i \star}, \hat{\theta}_{t}^{j j \star}, \hat{\theta}_{t}^{i j \star}, \hat{\theta}_{t}^{j i \star}, \hat{e}_{t}^{i i \star}, \hat{e}_{t}^{j j \star}, \hat{e}_{t}^{i j \star}, \hat{e}_{t}^{j i \star}$, it is easy to see that $A_{0}=0$. Therefore, a second-order approximation to the utility function simply yields

$$
\begin{aligned}
W \simeq & \frac{1}{2} \mathbf{E}_{\mathbf{0}} \sum_{t=0}^{\infty} \beta^{t}\left\{\Gamma_{e}^{i i}\left[\left(\tilde{e}_{t}^{i i}\right)^{2}+\left(\tilde{e}_{t}^{j j}\right)^{2}\right]+\Gamma_{e}^{i j}\left[\left(\tilde{e}_{t}^{i j}\right)^{2}+\left(\tilde{e}_{t}^{j i}\right)^{2}\right]+\right. \\
& \Gamma_{\theta}^{i i}\left[\left(\tilde{\theta}_{t}^{i i}\right)^{2}+\left(\tilde{\theta}_{t}^{j j}\right)^{2}\right]+\Gamma_{\theta}^{i j}\left[\left(\tilde{\theta}_{t}^{i j}\right)^{2}+\left(\tilde{\theta}_{t}^{j i}\right)^{2}\right]+ \\
& \Gamma_{c}\left[\left(\tilde{c}_{t}^{i}\right)^{2}+\left(\tilde{c}_{t}^{j}\right)^{2}\right]+\Gamma_{c_{H}}\left[\left(\tilde{c}_{H, t}^{i}\right)^{2}+\left(\tilde{c}_{H, t}^{j}\right)^{2}\right]+\Gamma_{c_{F}}\left[\left(\tilde{c}_{F, t}^{i}\right)^{2}+\left(\tilde{c}_{F, t}^{j}\right)^{2}\right]+ \\
& \left.\Gamma_{l}\left[\left(\tilde{l}_{t}^{i}\right)^{2}+\left(\tilde{l}_{t}^{j}\right)^{2}\right]+\Gamma_{\Delta}\left[\tilde{\Delta}_{t}^{i}+\tilde{\Delta}_{t}^{j}\right]\right\}+ \\
& \mathbf{E}_{\mathbf{0}} \sum_{t=0}^{\infty} \beta^{t}\left\{\Gamma_{3}^{i i}\left\{\tilde{\theta}_{t}^{i i}\left[\tilde{e}_{t}^{i i} \Gamma_{4}-(1-\rho) \tilde{e}_{t-1}^{i i}\right]+\tilde{\theta}_{t}^{j j}\left[\tilde{e}_{t}^{j j} \Gamma_{4}-(1-\rho) \tilde{e}_{t-1}^{j j}\right]\right\}+\right. \\
& \Gamma_{3}^{i j}\left\{\tilde{\theta}_{t}^{i j}\left[\tilde{e}_{t}^{i j} \Gamma_{4}-(1-\rho) \tilde{e}_{t-1}^{i j}\right]+\tilde{\theta}_{t}^{j i}\left[\tilde{e}_{t}^{j i} \Gamma_{4}-(1-\rho) \tilde{e}_{t-1}^{j i}\right]\right\}+ \\
& \left.\Gamma_{e^{i i}}{ }^{j i}\left(\tilde{e}_{t}^{i i} \tilde{e}_{t}^{j i}+\tilde{e}_{t}^{j j} \tilde{e}_{t}^{i j}\right)\right\}+t . i . p .
\end{aligned}
$$

Recall that up to the second order,

$$
\Delta_{t}^{i} \equiv \int_{0}^{1}\left(\frac{P_{H, t}^{i}(l)}{P_{H, t}}\right)^{-\frac{\varepsilon}{1-\alpha}} d l \approx 1+\frac{1}{2} \frac{\varepsilon}{1-\alpha} \frac{1-\alpha+\alpha \varepsilon}{1-\alpha} \operatorname{Var}_{l} \log \left(P_{H, t}^{i}(l)\right)
$$

such that

$$
\tilde{\Delta}_{t}^{i} \approx \frac{1}{2} \frac{\varepsilon}{1-\alpha} \frac{1-\alpha+\alpha \varepsilon}{1-\alpha} \operatorname{Var}_{l} \log \left(P_{H, t}^{i}(l)\right) .
$$

From Woodford (2011) we know that

$$
\sum_{t=0}^{\infty} \beta^{t} \operatorname{Var}_{l} \log \left(P_{H, t}^{i}(l)\right)=\sum_{t=0}^{\infty} \beta^{t} \frac{\delta}{(1-\delta)(1-\beta \delta)}\left(\hat{\pi}_{H, t}^{i}\right)^{2}
$$

so that

$$
\sum_{t=0}^{\infty} \beta^{t} \Gamma_{\Delta} \tilde{\Delta}_{t}^{i}=\sum_{t=0}^{\infty} \beta^{t} \Gamma_{\pi_{H}^{i}}\left(\hat{\pi}_{H, t}^{i}\right)^{2}=\sum_{t=0}^{\infty} \beta^{t} \Gamma_{\pi_{H}^{i}}\left(\tilde{\pi}_{H, t}^{i}\right)^{2}
$$

where

$$
\Gamma_{\pi_{H}^{i}} \equiv-\frac{\varepsilon}{\lambda^{i}} \frac{E^{1-\alpha}}{C}=-\frac{\varepsilon}{\lambda^{i}} \frac{Y}{C}
$$


Finally, the laws of motion for each of the four types of employment allow us to simplify the cross-terms as follows:

$$
\begin{aligned}
\mathbf{E}_{\mathbf{0}} \sum_{t=0}^{\infty} \beta^{t}\{ & \Gamma_{3}^{i i} \tilde{\theta}_{t}^{i i}\left[\frac{f}{\zeta(1-f)} \tilde{e}_{t}^{i i}+\tilde{e}_{t}^{i i}-(1-\rho) \tilde{e}_{t-1}^{i i}\right]+\Gamma_{3}^{i i} \tilde{\theta}_{t}^{j j}\left[\frac{f}{\zeta(1-f)} \tilde{e}_{t}^{j j}+\tilde{e}_{t}^{j j}-(1-\rho) \tilde{e}_{t-1}^{j j}\right]+ \\
& \Gamma_{3}^{i j} \tilde{\theta}_{t}^{i j}\left[\frac{f}{\zeta(1-f)} \tilde{e}_{t}^{i j}+\tilde{e}_{t}^{i j}-(1-\rho) \tilde{e}_{t-1}^{i j}\right]+\Gamma_{3}^{i j} \tilde{\theta}_{t}^{j i}\left[\frac{f}{\zeta(1-f)} \tilde{e}_{t}^{j i}+\tilde{e}_{t}^{j i}-(1-\rho) \tilde{e}_{t-1}^{j i}\right]+ \\
& \left.\Gamma_{e^{i i} e^{j i}}\left(\tilde{e}_{t}^{i i} \tilde{e}_{t}^{j i}+\tilde{e}_{t}^{j j} \tilde{e}_{t}^{i j}\right)\right\}= \\
\mathbf{E}_{\mathbf{0}} \sum_{t=0}^{\infty} \beta^{t}\{ & \Gamma_{3}^{i i} \rho \gamma\left[\left(\tilde{\theta}_{t}^{i i}\right)^{2}+\left(\tilde{\theta}_{t}^{j j}\right)^{2}\right]+\Gamma_{3}^{i j} \rho \gamma\left[\left(\tilde{\theta}_{t}^{i j}\right)^{2}+\left(\tilde{\theta}_{t}^{j i}\right)^{2}\right]+ \\
& \frac{\Gamma_{5} f}{\zeta(1-f)}\left[\left(1-\alpha_{1}\right)\left(\tilde{\theta}_{t}^{i i} \tilde{e}_{t}^{i i}+\tilde{\theta}_{t}^{j j} \tilde{e}_{t}^{j j}\right)+\alpha_{1}\left(\tilde{\theta}_{t}^{i j} \tilde{e}_{t}^{i j}+\tilde{\theta}_{t}^{j i} \tilde{e}_{t}^{j i}\right)\right]+ \\
& \Gamma_{5} \rho\left(1-\alpha_{1}\right)\left[\tilde{\omega}_{t}^{i}\left(\tilde{\theta}_{t}^{i i}-\tilde{\theta}_{t}^{i j}\right)+\tilde{\omega}_{t}^{j}\left(\tilde{\theta}_{t}^{j j}-\tilde{\theta}_{t}^{j i}\right)\right]- \\
& \Gamma_{5} f(1-\rho)\left(1-\alpha_{1}\right)\left[\left(1-\alpha_{1}\right)\left(\tilde{\theta}_{t}^{i i} \tilde{e}_{t-1}^{i i}+\tilde{\theta}_{t}^{j j} \tilde{e}_{t-1}^{j j}\right)+\alpha_{1}\left(\tilde{\theta}_{t}^{i i} \tilde{e}_{t-1}^{i j}+\tilde{\theta}_{t}^{j j} \tilde{e}_{t-1}^{j i}\right)\right]- \\
& \Gamma_{5} f(1-\rho) \alpha_{1}\left[\left(1-\alpha_{1}\right)\left(\tilde{\theta}_{t}^{i j} \tilde{e}_{t-1}^{i i}+\tilde{\theta}_{t}^{j i} \tilde{e}_{t-1}^{j j}\right)+\alpha_{1}\left(\tilde{\theta}_{t}^{i j} \tilde{e}_{t-1}^{i j}+\tilde{\theta}_{t}^{j i} \tilde{e}_{t-1}^{j i}\right)\right]+ \\
& \Gamma_{\left.e^{i i} e^{j i}\left(\tilde{e}_{t}^{i i} \tilde{e}_{t}^{j i}+\tilde{e}_{t}^{j j} \tilde{e}_{t}^{i j}\right)\right\}+t . i \cdot p .}
\end{aligned}
$$

where

$$
\Gamma_{5} \equiv-\frac{1}{\nu_{1}} \frac{E}{C} \frac{\kappa}{q} \frac{\rho \zeta(1-f)(1-\gamma)}{f+\zeta \rho(1-f)},
$$

such that a second-order approximation to the utility function in the model with labor 
mobility reads as follows:

$$
\begin{aligned}
W \simeq & \frac{1}{2} \mathbf{E}_{\mathbf{0}} \sum_{t=0}^{\infty} \beta^{t}\left\{\Gamma_{e}^{i i}\left[\left(\tilde{e}_{t}^{i i}\right)^{2}+\left(\tilde{e}_{t}^{j j}\right)^{2}\right]+\Gamma_{e}^{i j}\left[\left(\tilde{e}_{t}^{i j}\right)^{2}+\left(\tilde{e}_{t}^{j i}\right)^{2}\right]+\right. \\
& \Gamma_{\theta^{i i}}\left[\left(\tilde{\theta}_{t}^{i i}\right)^{2}+\left(\tilde{\theta}_{t}^{j j}\right)^{2}\right]+\Gamma_{\theta^{i j}}\left[\left(\tilde{\theta}_{t}^{i j}\right)^{2}+\left(\tilde{\theta}_{t}^{j i}\right)^{2}\right]+ \\
& \Gamma_{c}\left[\left(\tilde{c}_{t}^{i}\right)^{2}+\left(\tilde{c}_{t}^{j}\right)^{2}\right]+\Gamma_{c_{H}}\left[\left(\tilde{c}_{H, t}^{i}\right)^{2}+\left(\tilde{c}_{H, t}^{j}\right)^{2}\right]+\Gamma_{c_{F}}\left[\left(\tilde{c}_{F, t}^{i}\right)^{2}+\left(\tilde{c}_{F, t}^{j}\right)^{2}\right]+ \\
& \left.\Gamma_{l}\left[\left(\tilde{l}_{t}^{i}\right)^{2}+\left(\tilde{l}_{t}^{j}\right)^{2}\right]+\Gamma_{\pi_{H}^{i}}\left(\tilde{\pi}_{H, t}^{i}\right)^{2}+\Gamma_{\pi_{H}^{j}}\left(\tilde{\pi}_{H, t}^{j}\right)^{2}\right\}+ \\
& \mathbf{E}_{\mathbf{0}} \sum_{t=0}^{\infty} \beta^{t} \Gamma_{\theta e}\left\{\left(1-\alpha_{1}\right)\left(\tilde{\theta}_{t}^{i i} \tilde{e}_{t}^{i i}+\tilde{\theta}_{t}^{j j} \tilde{e}_{t}^{j j}\right)+\alpha_{1}\left(\tilde{\theta}_{t}^{i j} \tilde{e}_{t}^{i j}+\tilde{\theta}_{t}^{j i} \tilde{e}_{t}^{j i}\right)+\right. \\
& \left(1-\alpha_{1}\right) \frac{\zeta \rho(1-f)}{f}\left[\tilde{\omega}_{t}^{i}\left(\tilde{\theta}_{t}^{i i}-\tilde{\theta}_{t}^{i j}\right)+\tilde{\omega}_{t}^{j}\left(\tilde{\theta}_{t}^{j j}-\tilde{\theta}_{t}^{j i}\right)\right]- \\
& \zeta(1-\rho)(1-f)\left[\left(1-\alpha_{1}\right)^{2}\left(\tilde{\theta}_{t}^{i i} \tilde{e}_{t-1}^{i i}+\tilde{\theta}_{t}^{j j} \tilde{e}_{t-1}^{j j}\right)+\alpha_{1}^{2}\left(\tilde{\theta}_{t}^{i i} \tilde{e}_{t-1}^{i j}+\tilde{\theta}_{t}^{j j} \tilde{e}_{t-1}^{j i}\right)\right]- \\
& \zeta(1-\rho)(1-f)\left[\alpha_{1}\left(1-\alpha_{1}\right)\left(\tilde{\theta}_{t}^{i j} \tilde{e}_{t-1}^{i i}+\tilde{\theta}_{t}^{j i} \tilde{e}_{t-1}^{j j}+\tilde{\theta}_{t}^{i j} \tilde{e}_{t-1}^{i j}+\tilde{\theta}_{t}^{j i} \tilde{e}_{t-1}^{j i}\right)\right]+ \\
& \frac{\left.\Gamma_{e^{i i} e^{j i}}\left(\tilde{e}_{t}^{i i} \tilde{e}_{t}^{j i}+\tilde{e}_{t}^{j j} \tilde{e}_{t}^{i j}\right)\right\}+t . i \cdot p .,}{\Gamma_{\theta e}}
\end{aligned}
$$

where

$$
\begin{aligned}
\Gamma_{\theta^{i i}} & \equiv \Gamma_{\theta}^{i i}+2 \Gamma_{3}^{i i} \rho \gamma=-\frac{E}{C} \frac{\kappa}{q} \rho(1-\gamma)\left(1-\alpha_{1}\right)\left[1-\frac{1}{\nu_{1}} \frac{\gamma \zeta \rho(1-2(1-f))}{f+\zeta \rho(1-f)}\right] \\
\Gamma_{\theta^{i j}} & \equiv \Gamma_{\theta}^{i j}+2 \Gamma_{3}^{i j} \rho \gamma=-\frac{E}{C} \frac{\kappa}{q} \rho(1-\gamma) \alpha_{1}\left[1-\frac{1}{\nu_{1}} \frac{\gamma \zeta \rho(1-2(1-f))}{f+\zeta \rho(1-f)}\right] \\
\Gamma_{\theta e} & \equiv-\frac{1}{\nu_{1}} \frac{E}{C} \frac{\kappa}{q} \frac{\rho f(1-\gamma)}{f+\zeta \rho(1-f)} .
\end{aligned}
$$

\section{Appendix H. Optimal policy problem with mobility}

While in the benchmark no-mobility version of our model a zero-inflation policy implements the efficient allocation, the presence of a dynamic inefficiency related to households, migration decisions prevents this from happening in the full version of our model. Moreover, allowing for bargaining shocks leads to an additional trade-off between labor market tightness and inflation that the monetary authority needs to resolve. In what follows, we compute the optimal monetary policy for this general case.

Appendix H.1. Optimal policy problem

The optimal policy problem that the monetary authority solves consists in maximizing (G.26), subject to the following set of constraints: 
- Laws of motion for all four types of employment

- Household i's Euler equation and international risk-sharing condition

- New Keynesian Phillips curve for both regions $i$ and $j$

- Resource constraints for both regions $i$ and $j$

- Migration decision for both regions $i$ and $j$

- Total consumption aggregator for both regions $i$ and $j$

- Relation between domestic consumption, aggregate consumption, and relative prices for both regions $i$ and $j$

- Relation between domestic inflation rates and relative prices in both regions $i$ and $j$

Specifically, the central bank chooses the following sequence of variables:

$$
\left\{\tilde{\pi}_{H, t}^{i}, \tilde{\pi}_{H, t}^{j}, \tilde{c}_{t}^{i}, \tilde{c}_{t}^{j}, \tilde{r}_{t}^{i}, \tilde{\mathrm{q}}_{t}, \tilde{\theta}_{t}^{i i}, \tilde{\theta}_{t}^{i j}, \tilde{\theta}_{t}^{j j}, \tilde{\theta}_{t}^{j i}, \tilde{e}_{t}^{i i}, \tilde{e}_{t}^{i j}, \tilde{e}_{t}^{j j}, \tilde{e}_{t}^{j i}, \tilde{l}_{t}^{i}, \tilde{l}_{t}^{j}, \tilde{\omega}_{t}^{i}, \tilde{\omega}_{t}^{j}, \tilde{c}_{H, t}^{i}, \tilde{c}_{H, t}^{j}, \tilde{c}_{F, t}^{i}, \tilde{c}_{F, t}^{j}\right\}_{t=0}^{\infty}
$$

so as to maximize union-wide welfare

$$
\begin{aligned}
W \simeq & \frac{1}{2} \mathbf{E}_{\mathbf{0}} \sum_{t=0}^{\infty} \beta^{t}\left\{\Gamma_{e}^{i i}\left[\left(\tilde{e}_{t}^{i i}\right)^{2}+\left(\tilde{e}_{t}^{j j}\right)^{2}\right]+\Gamma_{e}^{i j}\left[\left(\tilde{e}_{t}^{i j}\right)^{2}+\left(\tilde{e}_{t}^{j i}\right)^{2}\right]+\right. \\
& \Gamma_{\theta^{i i}}\left[\left(\tilde{\theta}_{t}^{i i}\right)^{2}+\left(\tilde{\theta}_{t}^{j j}\right)^{2}\right]+\Gamma_{\theta^{i j}}\left[\left(\tilde{\theta}_{t}^{i j}\right)^{2}+\left(\tilde{\theta}_{t}^{j i}\right)^{2}\right]+ \\
& \Gamma_{c}\left[\left(\tilde{c}_{t}^{i}\right)^{2}+\left(\tilde{c}_{t}^{j}\right)^{2}\right]+\Gamma_{c_{H}}\left[\left(\tilde{c}_{H, t}^{i}\right)^{2}+\left(\tilde{c}_{H, t}^{j}\right)^{2}\right]+\Gamma_{c_{F}}\left[\left(\tilde{c}_{F, t}^{i}\right)^{2}+\left(\tilde{c}_{F, t}^{j}\right)^{2}\right]+ \\
& \left.\Gamma_{l}\left[\left(\tilde{l}_{t}^{i}\right)^{2}+\left(\tilde{l}_{t}^{j}\right)^{2}\right]+\Gamma_{\pi_{H}^{i}}\left(\tilde{\pi}_{H, t}^{i}\right)^{2}+\Gamma_{\pi_{H}^{j}}\left(\tilde{\pi}_{H, t}^{j}\right)^{2}\right\}+ \\
& \mathbf{E}_{\mathbf{0}} \sum_{t=0}^{\infty} \beta^{t} \Gamma_{\theta e}\left\{\left(1-\alpha_{1}\right)\left(\tilde{\theta}_{t}^{i i} \tilde{e}_{t}^{i i}+\tilde{\theta}_{t}^{j j} \tilde{e}_{t}^{j j}\right)+\alpha_{1}\left(\tilde{\theta}_{t}^{i j} \tilde{e}_{t}^{i j}+\tilde{\theta}_{t}^{j i} \tilde{e}_{t}^{j i}\right)+\right. \\
& \left(1-\alpha_{1}\right) \frac{\zeta \rho(1-f)}{f}\left[\tilde{\omega}_{t}^{i}\left(\tilde{\theta}_{t}^{i i}-\tilde{\theta}_{t}^{i j}\right)+\tilde{\omega}_{t}^{j}\left(\tilde{\theta}_{t}^{j j}-\tilde{\theta}_{t}^{j i}\right)\right]- \\
& \zeta(1-\rho)(1-f)\left[\left(1-\alpha_{1}\right)^{2}\left(\tilde{\theta}_{t}^{i i} \tilde{e}_{t-1}^{i i}+\tilde{\theta}_{t}^{j j} \tilde{e}_{t-1}^{j j}\right)+\alpha_{1}^{2}\left(\tilde{\theta}_{t}^{i i} \tilde{e}_{t-1}^{i j}+\tilde{\theta}_{t}^{j j} \tilde{e}_{t-1}^{j i}\right)\right]- \\
& \zeta(1-\rho)(1-f)\left[\alpha_{1}\left(1-\alpha_{1}\right)\left(\tilde{\theta}_{t}^{i j} \tilde{e}_{t-1}^{i i}+\tilde{\theta}_{t}^{j i} \tilde{e}_{t-1}^{j j}+\tilde{\theta}_{t}^{i j} \tilde{e}_{t-1}^{i j}+\tilde{\theta}_{t}^{j i} \tilde{e}_{t-1}^{j i}\right)\right]+ \\
& \left.\frac{\Gamma_{e^{i i}} e^{j i}}{\Gamma_{\theta e}}\left(\tilde{e}_{t}^{i i} \tilde{e}_{t}^{j i}+\tilde{e}_{t}^{j j} \tilde{e}_{t}^{i j}\right)\right\}+t . i \cdot p .
\end{aligned}
$$

subject to 
- $\lambda_{1, t}^{i i}$

$$
\tilde{\theta}_{t}^{i i}=\frac{\tilde{e}_{t}^{i i}}{\rho \gamma}-\frac{1-\rho}{\rho \gamma}\left[\left(1-f\left(1-\alpha_{1}\right)\right) \tilde{e}_{t-1}^{i i}-f \alpha_{1} \tilde{e}_{t-1}^{i j}\right]-\frac{\tilde{\omega}_{t}^{i}}{\gamma}
$$

- $\lambda_{1, t}^{i j}$

$$
\tilde{\theta}_{t}^{i j}=\frac{\tilde{e}_{t}^{i j}}{\rho \gamma}-\frac{1-\rho}{\rho \gamma}\left[\left(1-f \alpha_{1}\right) \tilde{e}_{t-1}^{i j}-f\left(1-\alpha_{1}\right) \tilde{e}_{t-1}^{i i}\right]+\frac{1-\alpha_{1}}{\alpha_{1}} \frac{\tilde{\omega}_{t}^{i}}{\gamma}
$$

- $\lambda_{1, t}^{j j}$

$$
\tilde{\theta}_{t}^{j j}=\frac{\tilde{e}_{t}^{j j}}{\rho \gamma}-\frac{1-\rho}{\rho \gamma}\left[\left(1-f\left(1-\alpha_{1}\right)\right) \tilde{e}_{t-1}^{j j}-f \alpha_{1} \tilde{e}_{t-1}^{j i}\right]-\frac{\tilde{\omega}_{t}^{j}}{\gamma}
$$

- $\lambda_{1, t}^{j i}$

$$
\tilde{\theta}_{t}^{j i}=\frac{\tilde{e}_{t}^{j i}}{\rho \gamma}-\frac{1-\rho}{\rho \gamma}\left[\left(1-f \alpha_{1}\right) \tilde{e}_{t-1}^{j i}-f\left(1-\alpha_{1}\right) \tilde{e}_{t-1}^{j j}\right]+\frac{1-\alpha_{1}}{\alpha_{1}} \frac{\tilde{\omega}_{t}^{j}}{\gamma}
$$

- $\lambda_{2, t}^{i}$

$$
\tilde{c}_{t}^{i}=\mathbf{E}_{\mathbf{t}}\left\{\tilde{c}_{t+1}^{i}\right\}-\tilde{r} r_{t}^{i}
$$

- $\lambda_{2, t}^{j}$

$$
\tilde{\mathrm{q}}_{t}=\tilde{c}_{t}^{i}-\tilde{c}_{t}^{j}
$$


- $\lambda_{3, t}^{i}$

$$
\begin{aligned}
\tilde{\pi}_{H, t}^{i}= & \beta \mathbf{E}_{\mathbf{t}}\left\{\tilde{\pi}_{H, t+1}^{i}\right\}+\lambda^{i} \frac{E^{\alpha}}{1-\alpha}\left[\frac{\kappa}{q} \frac{1-\gamma}{\gamma}+\frac{1}{\nu_{1}}(1-\zeta) \frac{1-b}{b} \frac{C}{l} \frac{\zeta \rho \gamma}{f+\rho \zeta(1-f)}\right] \tilde{\theta}_{t}^{i i} \\
& -\lambda^{i} \frac{E^{\alpha}}{1-\alpha} \beta(1-\rho) \frac{\kappa}{q} \frac{1-\gamma}{\gamma}(1-f) \mathbf{E}_{\mathbf{t}}\left\{\tilde{\theta}_{t+1}^{i i}\right\} \\
& +\lambda^{i} \frac{E^{\alpha}}{1-\alpha} \beta(1-\rho) \frac{\kappa}{q \gamma}[1-f(1-\gamma)] \tilde{r r}_{t}^{i} \\
& -\lambda^{i} \frac{E^{\alpha}}{1-\alpha} \beta(1-\rho) \frac{\kappa}{q \gamma}[1-f(1-\gamma)] \frac{1}{\nu_{2}} \mathbf{E}_{\mathbf{t}}\left\{\tilde{c}_{t+1}^{i}-\tilde{c}_{H, t+1}^{i}\right\} \\
& +\lambda^{i} \frac{E^{\alpha}}{1-\alpha}(1-\zeta) \frac{1-b}{b} \frac{C}{l}\left[\tilde{c}_{t}^{i}-\tilde{l}_{t}^{i}\left(1+\frac{1}{\nu_{1}} \frac{l}{1-l}\right)\right] \\
& +\lambda^{i} \frac{E^{\alpha}}{1-\alpha} \frac{\kappa}{q \gamma} \frac{1}{\nu_{2}}\left(\tilde{c}_{t}^{i}-\tilde{c}_{H, t}^{i}\right)+\lambda^{i} \alpha \alpha_{1} \tilde{e}_{t}^{j i} \\
& +\lambda^{i} \frac{E^{\alpha}}{1-\alpha}\left[\frac{\alpha(1-\alpha)\left(1-\alpha \alpha_{1}\right)}{E^{\alpha}}-\frac{1}{\nu_{1}}(1-\zeta) \frac{1-b}{b} \frac{C}{l} \frac{f+\zeta(1-f)}{f+\zeta \rho(1-f)}\right] \tilde{e}_{t}^{i i} \\
& +\lambda^{i} \frac{E^{\alpha}}{1-\alpha} \frac{1}{\nu_{1}}(1-\zeta) \frac{1-b}{b} \frac{C}{l} \frac{\zeta(1-\rho)(1-f)}{f+\rho \zeta(1-f)} \tilde{e}_{t-1}^{i i} \\
& +\lambda^{i} \frac{\alpha_{2}}{1-2 \alpha} \tilde{q}_{t}-\lambda^{i} \frac{E^{\alpha}}{1-\alpha} \frac{\kappa}{q \gamma^{2}}[1-\beta(1-\rho)(1-f)] \hat{\eta}_{t}^{i}
\end{aligned}
$$

- $\lambda_{3, t}^{j}$

$$
\begin{aligned}
\tilde{\pi}_{H, t}^{j}= & \beta \mathbf{E}_{\mathbf{t}}\left\{\tilde{\pi}_{H, t+1}^{j}\right\}+\lambda^{j} \frac{E^{\alpha}}{1-\alpha}\left[\frac{\kappa}{q} \frac{1-\gamma}{\gamma}+\frac{1}{\nu_{1}}(1-\zeta) \frac{1-b}{b} \frac{C}{l} \frac{\zeta \rho \gamma}{f+\rho \zeta(1-f)}\right] \tilde{\theta}_{t}^{j j} \\
& -\lambda^{j} \frac{E^{\alpha}}{1-\alpha} \beta(1-\rho) \frac{\kappa}{q} \frac{-\gamma}{\gamma}(1-f) \mathbf{E}_{\mathbf{t}}\left\{\tilde{\theta}_{t+1}^{j j}\right\} \\
& +\lambda^{j} \frac{E^{\alpha}}{1-\alpha} \beta(1-\rho) \frac{\kappa}{q \gamma}[1-f(1-\gamma)]\left(\tilde{r r}_{t}^{i}+\tilde{\mathrm{q}}_{t}-\tilde{\mathrm{q}}_{t+1}\right) \\
& -\lambda^{j} \frac{E^{\alpha}}{1-\alpha} \beta(1-\rho) \frac{\kappa}{q \gamma}[1-f(1-\gamma)] \frac{1}{\nu_{2}} \mathbf{E}_{\mathbf{t}}\left\{\widetilde{c_{t+1}^{j}}-\widetilde{c_{H, t+1}^{j}}\right\} \\
& +\lambda^{j} \frac{E^{\alpha}}{1-\alpha}(1-\zeta) \frac{1-b}{b} \frac{C}{l}\left[\tilde{c}_{t}^{j}-\tilde{l}_{t}^{j}\left(1+\frac{1}{\nu_{1}} \frac{l}{1-l}\right)\right] \\
& +\lambda^{j} \frac{E^{\alpha}}{1-\alpha} \frac{\kappa}{q \gamma} \frac{1}{\nu_{2}}\left(\tilde{c}_{t}^{j}-\tilde{c}_{H, t}^{j}\right)+\lambda^{j} \alpha \alpha_{1} \tilde{e}_{t}^{i j} \\
& +\lambda^{j} \frac{E^{\alpha}}{1-\alpha}\left[\frac{\alpha(1-\alpha)(1-\alpha)}{E^{\alpha}}-\frac{1}{\nu_{1}}(1-\zeta) \frac{1-b}{b} \frac{C}{l} \frac{f+\zeta(1-f)}{f+\zeta \rho(1-f)}\right] \tilde{e}_{t}^{j j} \\
& +\lambda^{j} \frac{E^{\alpha}}{1-\alpha} \frac{1}{\nu_{1}}(1-\zeta) \frac{1-b}{b} \frac{C}{l} \frac{\zeta(1-\rho)(1-f)}{f+\rho \zeta(1-f)} \tilde{e}_{t-1}^{j j} \\
& -\lambda^{j} \frac{\alpha_{2}}{1-2 \alpha} \tilde{\mathrm{q}}_{t}-\lambda^{j} \frac{E^{\alpha}}{1-\alpha} \frac{\kappa}{q \gamma^{2}}[1-\beta(1-\rho)(1-f)] \hat{\eta}_{t}^{j}
\end{aligned}
$$


- $\lambda_{4, t}^{i}$

$$
\begin{aligned}
\left(1-\alpha_{2}\right) \tilde{c}_{H, t}^{i}+\alpha_{2} \tilde{c}_{F, t}^{j}= & \left(\frac{1-\alpha}{E^{\alpha}}-\frac{\kappa}{q}\right) \frac{E}{C}\left(1-\alpha_{1}\right) \tilde{e}_{t}^{i i}+\left(\frac{1-\alpha}{E^{\alpha}}-\frac{\kappa}{q}\right) \frac{E}{C} \alpha_{1} \tilde{e}_{t}^{j i} \\
& -\rho(1-\gamma) \frac{\kappa}{q} \frac{E}{C}\left(1-\alpha_{1}\right) \tilde{\theta}_{t}^{i i}-\rho(1-\gamma) \frac{\kappa}{q} \frac{E}{C} \alpha_{1} \tilde{\theta}_{t}^{j i} \\
& +(1-\rho) \frac{\kappa}{q} \frac{E}{C}\left(1-\alpha_{1}\right) \tilde{e}_{t-1}^{i i}+(1-\rho) \frac{\kappa}{q} \frac{E}{C} \alpha_{1} \tilde{e}_{t-1}^{j i}
\end{aligned}
$$

- $\lambda_{4, t}^{j}$

$$
\begin{aligned}
\left(1-\alpha_{2}\right) \tilde{c}_{H, t}^{j}+\alpha_{2} \tilde{c}_{F, t}^{i}= & \left(\frac{1-\alpha}{E^{\alpha}}-\frac{\kappa}{q}\right) \frac{E}{C}\left(1-\alpha_{1}\right) \tilde{e}_{t}^{j j}+\left(\frac{1-\alpha}{E^{\alpha}}-\frac{\kappa}{q}\right) \frac{E}{C} \alpha_{1} \tilde{e}_{t}^{i j} \\
& -\rho(1-\gamma) \frac{\kappa}{q} \frac{E}{C}\left(1-\alpha_{1}\right) \tilde{\theta}_{t}^{j j}-\rho(1-\gamma) \frac{\kappa}{q} \frac{E}{C} \alpha_{1} \tilde{\theta}_{t}^{i j} \\
& +(1-\rho) \frac{\kappa}{q} \frac{E}{C}\left(1-\alpha_{1}\right) \tilde{e}_{t-1}^{j j}+(1-\rho) \frac{\kappa}{q} \frac{E}{C} \alpha_{1} \tilde{e}_{t-1}^{i j}
\end{aligned}
$$

- $\lambda_{5, t}^{i}$

$$
\begin{aligned}
\tilde{l}_{t}^{i}= & -\left(1-\alpha_{1}\right) \frac{E}{l}\left(1+\zeta \frac{1-f}{f}\right) \tilde{e}_{t}^{i i}-\alpha_{1} \frac{E}{l}\left(1+\zeta \frac{1-f}{f}\right) \tilde{e}_{t}^{i j} \\
& +\left(1-\alpha_{1}\right) \frac{E}{l} \frac{\rho \zeta \gamma}{f} \tilde{\theta}_{t}^{i i}+\alpha_{1} \frac{E}{l} \frac{\rho \zeta \gamma}{f} \tilde{\theta}_{t}^{i j} \\
& +\left(1-\alpha_{1}\right) \frac{E}{l}(1-\rho) \zeta \frac{1-f}{f} \tilde{e}_{t-1}^{i i}+\alpha_{1} \frac{E}{l}(1-\rho) \zeta \frac{1-f}{f} \tilde{e}_{t-1}^{i j}
\end{aligned}
$$

- $\lambda_{5, t}^{j}$

$$
\begin{aligned}
\tilde{l}_{t}^{j}= & -\left(1-\alpha_{1}\right) \frac{E}{l}\left(1+\zeta \frac{1-f}{f}\right) \tilde{e}_{t}^{j j}-\alpha_{1} \frac{E}{l}\left(1+\zeta \frac{1-f}{f}\right) \tilde{e}_{t}^{j i} \\
& +\left(1-\alpha_{1}\right) \frac{E}{l} \frac{\rho \zeta \gamma}{f} \tilde{\theta}_{t}^{j j}+\alpha_{1} \frac{E}{l} \frac{\rho \zeta \gamma}{f} \tilde{\theta}_{t}^{j i} \\
& +\left(1-\alpha_{1}\right) \frac{E}{l}(1-\rho) \zeta \frac{1-f}{f} \tilde{e}_{t-1}^{j j}+\alpha_{1} \frac{E}{l}(1-\rho) \zeta \frac{1-f}{f} \tilde{e}_{t-1}^{j i}
\end{aligned}
$$

- $\lambda_{6, t}^{i}$

$$
\frac{\alpha_{2}}{2 \alpha_{2}-1} \tilde{\mathrm{q}}_{t}=\frac{1}{\nu_{2}}\left(\tilde{c}_{t}^{i}-\tilde{c}_{H, t}^{i}\right)
$$


- $\lambda_{6, t}^{j}$

$$
-\frac{\alpha_{2}}{2 \alpha_{2}-1} \tilde{\mathrm{q}}_{t}=\frac{1}{\nu_{2}}\left(\tilde{c}_{t}^{j}-\tilde{c}_{H, t}^{j}\right)
$$

- $\lambda_{7, t}^{i}$

$$
\tilde{c}_{t}^{i}=\left(1-\alpha_{2}\right) \tilde{c}_{H, t}^{i}+\alpha_{2} \tilde{c}_{F, t}^{i}
$$

- $\lambda_{7, t}^{j}$

$$
\tilde{c}_{t}^{j}=\left(1-\alpha_{2}\right) \tilde{c}_{H, t}^{j}+\alpha_{2} \tilde{c}_{F, t}^{j}
$$

- $\lambda_{8, t}$

$$
\tilde{\mathrm{q}}_{t}=\left(1-2 \alpha_{2}\right)\left(\tilde{\pi}_{H, t}^{j}-\tilde{\pi}_{H, t}^{i}\right)+\tilde{\mathrm{q}}_{t-1}-\hat{\mathrm{q}}_{t}^{\star}+\hat{\mathrm{q}}_{t-1}^{\star}
$$

- $\lambda_{9, t}^{i}$

$$
\begin{aligned}
& \frac{1}{\nu_{1}} \frac{1-b}{b} \frac{C}{l} \frac{\zeta}{f} \frac{f+\zeta(1-f)}{f+\rho \zeta(1-f)}\left(\tilde{e}_{t}^{i i}-\tilde{e}_{t}^{i j}\right)-\frac{1}{\nu_{1}} \frac{1-b}{b} \frac{C}{l} \frac{\zeta}{f} \frac{\zeta(1-\rho)(1-f)}{f+\rho \zeta(1-f)}\left(\tilde{e}_{t-1}^{i i}-\tilde{e}_{t-1}^{i j}\right) \\
& +\left[\frac{\kappa}{q \gamma}(1-\gamma)(1-\beta(1-\rho)(1-f) \gamma)-\frac{1}{\nu_{1}} \frac{1-b}{b} \frac{C}{l} \frac{\zeta}{f} \frac{\zeta \rho \gamma}{f+\rho \zeta(1-f)}\right]\left(\tilde{\theta}_{t}^{i i}-\tilde{\theta}_{t}^{i j}\right) \\
& -\frac{\kappa}{q \gamma}(1-\gamma) \beta(1-\rho)(1-f-\gamma)\left(\tilde{\theta}_{t+1}^{i i}-\tilde{\theta}_{t+1}^{i j}\right) \\
& +\frac{\kappa}{q \gamma}(1-\gamma) \frac{1}{\nu_{2}}\left(\tilde{c}_{t}^{i}-\tilde{c}_{H, t}^{i}-\tilde{c}_{t}^{j}+\tilde{c}_{H, t}^{j}\right) \\
& -\frac{\kappa}{q \gamma}(1-\gamma) \beta(1-\rho)(1-f) \frac{1}{\nu_{2}}\left(\tilde{c}_{t+1}^{i}-\tilde{c}_{H, t+1}^{i}-\tilde{c}_{t+1}^{j}+\tilde{c}_{H, t+1}^{j}\right) \\
& -\frac{\kappa}{q}(1-\gamma) \beta(1-\rho)(1-f)\left(\hat{\theta}_{t}^{i i \star}-\hat{\theta}_{t}^{i j \star}\right) \\
& -\frac{\kappa}{q \gamma}(1-\gamma) \beta(1-\rho)(1-f-\gamma)\left(\hat{\theta}_{t+1}^{i i \star}-\hat{\theta}_{t+1}^{i j \star}\right) \\
& -\frac{\kappa}{q \gamma}(1-\gamma) \beta(1-\rho)(1-f) \frac{1}{\nu_{2}}\left(\hat{c}_{t+1}^{i \star}-\hat{c}_{H, t+1}^{i \star}-\hat{c}_{t+1}^{j \star}+\hat{c}_{H, t+1}^{j \star}\right) \\
& -\Gamma_{\mathrm{q}}\left(\tilde{\mathrm{q}}_{t}+\hat{\mathrm{q}}_{t}^{\star}\right)-\frac{\kappa}{q \gamma}(1-\gamma) \beta(1-\rho)\left(\tilde{\mathrm{q}}_{t+1}+\hat{\mathrm{q}}_{t+1}^{\star}\right) \\
& -\frac{\kappa}{q \gamma^{2}}(1-\beta(1-\rho)(1-f))\left(\hat{\eta}_{t}^{i}-\hat{\eta}_{t}^{j}\right) \\
& -\left[\frac{1}{\nu_{1}} \frac{1-b}{b} \frac{C}{l} \frac{\zeta}{f} \frac{\zeta \rho}{f+\zeta \rho(1-f)}+\frac{\kappa}{q \gamma}(1-\gamma) \beta(1-\rho)(1-f)\right]\left(\hat{\tau}_{t}^{i}-\hat{\tau}_{t}^{j}\right) \\
& +\beta(1-\rho)(1-\gamma) \frac{\kappa}{q \gamma}\left(\hat{\tau}_{t+1}^{i}-\hat{\tau}_{t+1}^{j}\right)=0 \\
&
\end{aligned}
$$


- $\lambda_{9, t}^{j}$

$$
\begin{aligned}
& \frac{1}{\nu_{1}} \frac{1-b}{b} \frac{C}{l} \frac{\zeta}{f} \frac{f+\zeta(1-f)}{f+\rho \zeta(1-f)}\left(\tilde{e}_{t}^{j j}-\tilde{e}_{t}^{j i}\right)-\frac{1}{\nu_{1}} \frac{1-b}{b} \frac{C}{l} \frac{\zeta}{f} \frac{\zeta(1-\rho)(1-f)}{f+\rho \zeta(1-f)}\left(\tilde{e}_{t-1}^{j j}-\tilde{e}_{t-1}^{j i}\right) \\
& +\left[\frac{\kappa}{q \gamma}(1-\gamma)(1-\beta(1-\rho)(1-f) \gamma)-\frac{1}{\nu_{1}} \frac{1-b}{b} \frac{C}{l} \frac{\zeta}{f} \frac{\zeta \rho \gamma}{f+\rho \zeta(1-f)}\right]\left(\tilde{\theta}_{t}^{j j}-\tilde{\theta}_{t}^{j i}\right) \\
& -\frac{\kappa}{q \gamma}(1-\gamma) \beta(1-\rho)(1-f-\gamma)\left(\tilde{\theta}_{t+1}^{j j}-\tilde{\theta}_{t+1}^{j i}\right) \\
& +\frac{\kappa}{q \gamma}(1-\gamma) \frac{1}{\nu_{2}}\left(\tilde{c}_{t}^{j}-\tilde{c}_{H, t}^{j}-\tilde{c}_{t}^{i}+\tilde{c}_{H, t}^{i}\right) \\
& -\frac{\kappa}{q \gamma}(1-\gamma) \beta(1-\rho)(1-f) \frac{1}{\nu_{2}}\left(\tilde{c}_{t+1}^{j}-\tilde{c}_{H, t+1}^{j}-\tilde{c}_{t+1}^{i}+\tilde{c}_{H, t+1}^{i}\right) \\
& -\frac{\kappa}{q}(1-\gamma) \beta(1-\rho)(1-f)\left(\hat{\theta}_{t}^{j j \star}-\hat{\theta}_{t}^{j i \star}\right) \\
& -\frac{\kappa}{q \gamma}(1-\gamma) \beta(1-\rho)(1-f-\gamma)\left(\hat{\theta}_{t+1}^{j j \star}-\hat{\theta}_{t+1}^{j i \star}\right) \\
& -\frac{\kappa}{q \gamma}(1-\gamma) \beta(1-\rho)(1-f) \frac{1}{\nu_{2}}\left(\hat{c}_{t+1}^{j \star}-\hat{c}_{H, t+1}^{j \star}-\hat{c}_{t+1}^{i \star}+\hat{c}_{H, t+1}^{i \star}\right) \\
& +\Gamma_{\mathrm{q}}\left(\tilde{\mathrm{q}}_{t}+\hat{\mathrm{q}}_{t}^{\star}\right)+\frac{\kappa}{q \gamma}(1-\gamma) \beta(1-\rho)\left(\tilde{\mathrm{q}}_{t+1}+\hat{\mathrm{q}}_{t+1}^{\star}\right) \\
& -\frac{\kappa}{q \gamma^{2}}(1-\beta(1-\rho)(1-f))\left(\hat{\eta}_{t}^{j}-\hat{\eta}_{t}^{i}\right) \\
& -\left[\frac{1}{\nu_{1}} \frac{1-b}{b} \frac{C}{l} \frac{\zeta}{f} \frac{\zeta \rho}{f+\zeta \rho(1-f)}+\frac{\kappa}{q \gamma}(1-\gamma) \beta(1-\rho)(1-f)\right]\left(\hat{\tau}_{t}^{j}-\hat{\tau}_{t}^{i}\right) \\
& +\beta(1-\rho)(1-\gamma) \frac{\kappa}{q \gamma}\left(\hat{\tau}_{t+1}^{j}-\hat{\tau}_{t+1}^{i}\right)=0
\end{aligned}
$$

- $\lambda_{10, t}^{i}$

$$
\tilde{e}_{t}^{i i}+\frac{\alpha_{1}}{1-\alpha_{1}} \tilde{e}_{t}^{i j}-(1-f)(1-\rho)\left[\tilde{e}_{t-1}^{i i}+\frac{\alpha_{1}}{1-\alpha_{1}} \tilde{e}_{t-1}^{i j}\right]-\rho \gamma\left[\tilde{\theta}_{t}^{i i}+\frac{\alpha_{1}}{1-\alpha_{1}} \tilde{\theta}_{t}^{i j}\right]=0
$$

- $\lambda_{10, t}^{j}$

$$
\tilde{e}_{t}^{j j}+\frac{\alpha_{1}}{1-\alpha_{1}} \tilde{e}_{t}^{j i}-(1-f)(1-\rho)\left[\tilde{e}_{t-1}^{j j}+\frac{\alpha_{1}}{1-\alpha_{1}} \tilde{e}_{t-1}^{j i}\right]-\rho \gamma\left[\tilde{\theta}_{t}^{j j}+\frac{\alpha_{1}}{1-\alpha_{1}} \tilde{\theta}_{t}^{j i}\right]=0
$$

Appendix H.2. Optimality conditions

The first-order conditions of the optimal policy problem state as follows (terms in blue mark additional first-order conditions, or extra parts of given first-order conditions, relative to the benchmark open-economy case without mobility):

- $\tilde{\pi}_{H, t}^{i}$ :

$$
-\frac{\varepsilon}{\lambda^{i}} \frac{Y}{C} \tilde{\pi}_{H, t}^{i}+\lambda_{3, t-1}^{i}-\lambda_{3, t}^{i}-\left(1-2 \alpha_{2}\right) \lambda_{8, t}=0
$$


- $\tilde{\pi}_{H, t}^{j}:$

$$
-\frac{\varepsilon}{\lambda^{j}} \frac{Y}{C} \tilde{\pi}_{H, t}^{j}+\lambda_{3, t-1}^{j}-\lambda_{3, t}^{j}+\left(1-2 \alpha_{2}\right) \lambda_{8, t}=0
$$

- $\tilde{c}_{t}^{i}$ :

$$
\begin{aligned}
& \frac{1-\nu_{2}}{\nu_{2}} \tilde{c}_{t}^{i}-\lambda_{2, t}^{i}+\frac{\lambda_{2, t-1}^{i}}{\beta}+\lambda_{2, t}^{j}+\lambda^{i} \frac{E^{\alpha}}{1-\alpha}(1-\zeta) \frac{1-b}{b} \frac{C}{l} \lambda_{3, t}^{i}+ \\
& \frac{E^{\alpha}}{1-\alpha} \frac{\kappa}{q \gamma} \frac{\lambda^{i}}{\nu_{2}}\left[\lambda_{3, t}^{i}-\lambda_{3, t-1}^{i}(1-\rho)(1-f(1-\gamma))\right]+\frac{\lambda_{6, t}^{i}}{\nu_{2}}-\lambda_{7, t}^{i}+ \\
& \frac{1}{\nu_{2}} \frac{\kappa}{q \gamma}(1-\gamma)\left(\lambda_{9, t}^{i}-\lambda_{9, t-1}^{i}(1-\rho)(1-f)\right)- \\
& \frac{1}{\nu_{2}} \frac{\kappa}{q \gamma}(1-\gamma)\left(\lambda_{9, t}^{j}-\lambda_{9, t-1}^{j}(1-\rho)(1-f)\right)=0
\end{aligned}
$$

- $\tilde{c}_{t}^{j}$ :

$$
\begin{aligned}
& \frac{1-\nu_{2}}{\nu_{2}} \tilde{c}_{t}^{j}-\lambda_{2, t}^{j}+\lambda^{j} \frac{E^{\alpha}}{1-\alpha}(1-\zeta) \frac{1-b}{b} \frac{C}{l} \lambda_{3, t}^{j}+ \\
& \frac{E^{\alpha}}{1-\alpha} \frac{\kappa}{q \gamma} \frac{\lambda^{j}}{\nu_{2}}\left[\lambda_{3, t}^{j}-\lambda_{3, t-1}^{j}(1-\rho)(1-f(1-\gamma))\right]+\frac{\lambda_{6, t}^{j}}{\nu_{2}}-\lambda_{7, t}^{j}+ \\
& \frac{1}{\nu_{2}} \frac{\kappa}{q \gamma}(1-\gamma)\left(\lambda_{9, t}^{j}-\lambda_{9, t-1}^{j}(1-\rho)(1-f)\right)- \\
& \frac{1}{\nu_{2}} \frac{\kappa}{q \gamma}(1-\gamma)\left(\lambda_{9, t}^{i}-\lambda_{9, t-1}^{i}(1-\rho)(1-f)\right)=0
\end{aligned}
$$

- $\tilde{r r}_{t}^{i}$ :

$$
-\lambda_{2, t}^{i}+\frac{E^{\alpha}}{1-\alpha} \frac{\kappa}{q \gamma} \beta(1-\rho)[1-f(1-\gamma)]\left[\lambda^{i} \lambda_{3, t}^{i}+\lambda^{j} \lambda_{3, t}^{j}\right]=0
$$

- $\tilde{\mathrm{q}}_{t}$ :

$$
\begin{aligned}
& -\lambda_{2, t}^{j}+\frac{\alpha_{2}}{1-2 \alpha_{2}}\left[\lambda^{i} \lambda_{3, t}^{i}-\lambda^{j} \lambda_{3, t}^{j}\right]-\lambda^{j} \frac{E^{\alpha}}{1-\alpha} \frac{\kappa}{q \gamma}(1-\rho)[1-f(1-\gamma)]\left[\lambda_{3, t-1}^{j}-\beta \lambda_{3, t}^{j}\right] \\
& -\frac{\alpha_{2}}{2 \alpha_{2}-1}\left(\lambda_{6, t}^{i}-\lambda_{6, t}^{j}\right)-\lambda_{8, t}+\beta \lambda_{8, t+1} \\
& -\Gamma_{\mathrm{q}} \lambda_{9, t}^{i}-\frac{\kappa}{q \gamma}(1-\gamma)(1-\rho) \lambda_{9, t-1}^{i}+\Gamma_{\mathrm{q}} \lambda_{9, t}^{j}+\frac{\kappa}{q \gamma}(1-\gamma)(1-\rho) \lambda_{9, t-1}^{j}=0
\end{aligned}
$$


- $\tilde{\theta}_{t}^{i i}$ :

$$
\begin{aligned}
& -\left(1-\alpha_{1}\right) \frac{E}{C} \frac{\kappa}{q}(1-\gamma) \rho\left[1-\frac{1}{\nu_{1}} \frac{\gamma \zeta \rho(1-2(1-f))}{f+\zeta \rho(1-f)}\right] \tilde{\theta}_{t}^{i i}-\lambda_{1, t}^{i i} \\
& -\left[\frac{1-\alpha_{1}}{\nu_{1}} \frac{E}{C} \frac{\kappa}{q} \frac{\rho \zeta(1-f)(1-\gamma)}{f+\zeta \rho(1-f)}\right]\left\{\frac{f \tilde{e}_{t}^{i i}}{\zeta(1-f)}+\rho \tilde{\omega}_{t}^{i}-f(1-\rho)\left[\left(1-\alpha_{1}\right) \tilde{e}_{t-1}^{i i}+\alpha_{1} \tilde{e}_{t-1}^{i j}\right]\right\} \\
& +\lambda^{i} \frac{E^{\alpha}}{1-\alpha}\left[\lambda_{3, t}^{i}\left(\frac{\kappa}{q} \frac{1-\gamma}{\gamma}+\frac{1-\zeta}{\nu_{1}} \frac{1-b}{b} \frac{C}{l} \frac{\zeta \rho \gamma}{f+\zeta \rho(1-f)}\right)-\lambda_{3, t-1}^{i} \frac{\kappa}{q} \frac{1-\gamma}{\gamma}(1-\rho)(1-f)\right] \\
& -\lambda_{4, t}^{i} \rho(1-\gamma) \frac{\kappa}{q} \frac{E}{C}\left(1-\alpha_{1}\right)+\lambda_{5, t}^{i} \frac{\rho \zeta \gamma}{f} \frac{E}{l}\left(1-\alpha_{1}\right) \\
& +\lambda_{9, t}^{i}\left[\frac{\kappa}{q \gamma}(1-\gamma)(1-\beta(1-\rho)(1-f) \gamma)-\frac{1}{\nu_{1}} \frac{1-b}{b} \frac{C}{l} \frac{\zeta}{f} \frac{\zeta \rho \gamma}{f+\rho \zeta(1-f)}\right] \\
& -\lambda_{9, t-1}^{i} \frac{\kappa}{q \gamma}(1-\gamma)(1-\rho)(1-f-\gamma)-\lambda_{10, t}^{i} \rho \gamma=0
\end{aligned}
$$

- $\tilde{\theta}_{t}^{i j}$ :

$$
\begin{aligned}
& -\alpha_{1} \frac{E}{C} \frac{\kappa}{q}(1-\gamma) \rho\left[1-\frac{1}{\nu_{1}} \frac{\gamma \zeta \rho(1-2(1-f))}{f+\zeta \rho(1-f)}\right] \tilde{\theta}_{t}^{i j}-\lambda_{1, t}^{i j} \\
& -\left[\frac{\alpha_{1}}{\nu_{1}} \frac{E}{C} \frac{\kappa}{q} \frac{\rho \zeta(1-f)(1-\gamma)}{f+\zeta \rho(1-f)}\right]\left\{\frac{f \tilde{e}_{t}^{i j}}{\zeta(1-f)}+\frac{1-\alpha_{1}}{\alpha_{1}} \rho \tilde{\omega}_{t}^{i}-f(1-\rho)\left[\left(1-\alpha_{1}\right) \tilde{e}_{t-1}^{i i}+\alpha_{1} \tilde{e}_{t-1}^{i j}\right]\right\} \\
& -\lambda_{4, t}^{j} \rho(1-\gamma) \frac{\kappa}{q} \frac{E}{C} \alpha_{1}+\lambda_{5, t}^{i} \frac{\rho \zeta \gamma}{f} \frac{E}{l} \alpha_{1} \\
& -\lambda_{9, t}^{i}\left[\frac{\kappa}{q \gamma}(1-\gamma)(1-\beta(1-\rho)(1-f) \gamma)-\frac{1}{\nu_{1}} \frac{1-b}{b} \frac{C}{l} \frac{\zeta}{f} \frac{\zeta \rho \gamma}{f+\rho \zeta(1-f)}\right] \\
& +\lambda_{9, t-1}^{i} \frac{\kappa}{q \gamma}(1-\gamma)(1-\rho)(1-f-\gamma)-\lambda_{10, t}^{i} \rho \gamma \frac{\alpha_{1}}{1-\alpha_{1}}=0
\end{aligned}
$$

- $\tilde{\theta}_{t}^{j j}$ :

$$
\begin{aligned}
& -\left(1-\alpha_{1}\right) \frac{E}{C} \frac{\kappa}{q}(1-\gamma) \rho\left[1-\frac{1}{\nu_{1}} \frac{\gamma \zeta \rho(1-2(1-f))}{f+\zeta \rho(1-f)}\right] \tilde{\theta}_{t}^{j j}-\lambda_{1, t}^{j j} \\
& -\left[\frac{1-\alpha_{1}}{\nu_{1}} \frac{E}{C} \frac{\kappa}{q} \frac{\rho \zeta(1-f)(1-\gamma)}{f+\zeta \rho(1-f)}\right]\left\{\frac{f \tilde{e}_{t}^{j j}}{\zeta(1-f)}+\rho \tilde{\omega}_{t}^{j}-f(1-\rho)\left[\left(1-\alpha_{1}\right) \tilde{e}_{t-1}^{j j}+\alpha_{1} \tilde{e}_{t-1}^{j i}\right]\right\} \\
& +\lambda^{j} \frac{E^{\alpha}}{1-\alpha}\left[\lambda_{3, t}^{j}\left(\frac{\kappa}{q} \frac{1-\gamma}{\gamma}+\frac{1-\zeta}{\nu_{1}} \frac{1-b}{b} \frac{C}{l} \frac{\zeta \rho \gamma}{f+\zeta \rho(1-f)}\right)-\lambda_{3, t-1}^{j} \frac{\kappa}{q} \frac{1-\gamma}{\gamma}(1-\rho)(1-f)\right] \\
& -\lambda_{4, t}^{j} \rho(1-\gamma) \frac{\kappa}{q} \frac{E}{C}\left(1-\alpha_{1}\right)+\lambda_{5, t}^{j} \frac{\rho \zeta \gamma}{f} \frac{E}{l}\left(1-\alpha_{1}\right) \\
& +\lambda_{9, t}^{j}\left[\frac{\kappa}{q \gamma}(1-\gamma)(1-\beta(1-\rho)(1-f) \gamma)-\frac{1}{\nu_{1}} \frac{1-b}{b} \frac{C}{l} \frac{\zeta}{f} \frac{\zeta \rho \gamma}{f+\rho \zeta(1-f)}\right] \\
& -\lambda_{9, t-1}^{j} \frac{\kappa}{q \gamma}(1-\gamma)(1-\rho)(1-f-\gamma)-\lambda_{10, t}^{j} \rho \gamma=0
\end{aligned}
$$


- $\tilde{\theta}_{t}^{j i}$ :

$$
\begin{aligned}
& -\alpha_{1} \frac{E}{C} \frac{\kappa}{q}(1-\gamma) \rho\left[1-\frac{1}{\nu_{1}} \frac{\gamma \zeta \rho(1-2(1-f))}{f+\zeta \rho(1-f)}\right] \tilde{\theta}_{t}^{j i}-\lambda_{1, t}^{j i} \\
& -\left[\frac{\alpha_{1}}{\nu_{1}} \frac{E}{C} \frac{\kappa}{q} \frac{\rho \zeta(1-f)(1-\gamma)}{f+\zeta \rho(1-f)}\right]\left\{\frac{f \tilde{e}_{t}^{j i}}{\zeta(1-f)}+\frac{1-\alpha_{1}}{\alpha_{1}} \rho \tilde{\omega}_{t}^{j}-f(1-\rho)\left[\left(1-\alpha_{1}\right) \tilde{e}_{t-1}^{j j}+\alpha_{1} \tilde{e}_{t-1}^{j i}\right]\right\} \\
& -\lambda_{4, t}^{i} \rho(1-\gamma) \frac{\kappa}{q} \frac{E}{C} \alpha_{1}+\lambda_{5, t}^{j} \frac{\rho \zeta \gamma}{f} \frac{E}{l} \alpha_{1} \\
& -\lambda_{9, t}^{j}\left[\frac{\kappa}{q \gamma}(1-\gamma)(1-\beta(1-\rho)(1-f) \gamma)-\frac{1}{\nu_{1}} \frac{1-b}{b} \frac{C}{l} \frac{\zeta}{f} \frac{\zeta \rho \gamma}{f+\rho \zeta(1-f)}\right] \\
& +\lambda_{9, t-1}^{j} \frac{\kappa}{q \gamma}(1-\gamma)(1-\rho)(1-f-\gamma)-\lambda_{10, t}^{j} \rho \gamma \frac{\alpha_{1}}{1-\alpha_{1}}=0
\end{aligned}
$$

- $\tilde{e}_{t}^{i i}$ :

$$
\begin{aligned}
& \Gamma_{e}^{i i} \tilde{e}_{t}^{i i}+\Gamma_{e^{i i}} e_{i i}^{j i} \tilde{e}_{t}^{j i}+\Gamma_{\theta e} \frac{f\left(1-\alpha_{1}\right)}{\zeta(1-f)} \tilde{\theta}_{t}^{i i}-\beta \Gamma_{\theta e} f(1-\rho)\left(1-\alpha_{1}\right)\left[\left(1-\alpha_{1}\right) \tilde{\theta}_{t+1}^{i i}+\alpha_{1} \tilde{\theta}_{t+1}^{i j}\right] \\
& +\frac{\lambda_{1, t}^{i i}}{\rho \gamma}-\beta \frac{1-\rho}{\rho \gamma}\left[\lambda_{1, t+1}^{i i}\left(1-f\left(1-\alpha_{1}\right)\right)-\lambda_{1, t+1}^{i j} f\left(1-\alpha_{1}\right)\right] \\
& +\lambda^{i} \frac{E^{\alpha}}{1-\alpha} \lambda_{3, t}^{i}\left(\frac{\alpha(1-\alpha)\left(1-\alpha_{1}\right)}{E^{\alpha}}-\frac{1-b}{b} \frac{C}{l} \frac{1-\zeta}{\nu_{1}} \frac{f+\zeta(1-f)}{f+\zeta \rho(1-f)}\right) \\
& +\lambda^{i} \frac{E^{\alpha}}{1-\alpha} \beta \lambda_{3, t+1}^{i} \frac{1-b}{b} \frac{C}{l} \frac{1-\zeta}{\nu_{1}} \frac{\zeta(1-\rho)(1-f)}{f+\zeta \rho(1-f)} \\
& +\lambda_{4, t}^{i}\left(\frac{1-\alpha}{E^{\alpha}}-\frac{\kappa}{q}\right) \frac{E}{C}\left(1-\alpha_{1}\right)+\lambda_{4, t+1}^{i} \beta(1-\rho) \frac{\kappa}{q} \frac{E}{C}\left(1-\alpha_{1}\right) \\
& -\lambda_{5, t}^{i} \frac{E}{l}\left(1-\alpha_{1}\right)\left(1+\zeta \frac{1-f}{f}\right)+\lambda_{5, t+1}^{i} \beta(1-\rho) \zeta \frac{1-f}{f} \frac{E}{l}\left(1-\alpha_{1}\right) \\
& +\frac{1}{\nu_{1}} \frac{1-b}{b} \frac{C}{l} \frac{\zeta}{f}\left[\lambda_{9, t}^{i} \frac{f+\zeta(1-f)}{f+\zeta \rho(1-f)}-\beta \lambda_{9, t+1}^{i} \frac{\zeta(1-\rho)(1-f)}{f+\zeta \rho(1-f)}\right] \\
& +\lambda_{10, t}^{i}-\beta \lambda_{10, t+1}^{i}(1-f)(1-\rho)=0
\end{aligned}
$$


- $\tilde{e}_{t}^{i j}$ :

$$
\begin{aligned}
& \Gamma_{e}^{i j} \tilde{e}_{t}^{i j}+\Gamma_{e^{i i} e^{j i}} \tilde{e}_{t}^{j j}+\Gamma_{\theta e} \frac{f \alpha_{1}}{\zeta(1-f)} \tilde{\theta}_{t}^{i j}-\beta \Gamma_{\theta e} f(1-\rho) \alpha_{1}\left[\left(1-\alpha_{1}\right) \tilde{\theta}_{t+1}^{i i}+\alpha_{1} \tilde{\theta}_{t+1}^{i j}\right] \\
& +\frac{\lambda_{1, t}^{i j}}{\rho \gamma}-\beta \frac{1-\rho}{\rho \gamma}\left[\lambda_{1, t+1}^{i j}\left(1-f \alpha_{1}\right)-\lambda_{1, t+1}^{i i} f \alpha_{1}\right]+\lambda_{3, t^{j}}^{j} \alpha \alpha_{1} \\
& +\lambda_{4, t}^{j}\left(\frac{1-\alpha}{E^{\alpha}}-\frac{\kappa}{q}\right) \frac{E}{C} \alpha_{1}+\lambda_{4, t+1}^{j} \beta(1-\rho) \frac{\kappa}{q} \frac{E}{C} \alpha_{1} \\
& -\lambda_{5, t}^{i} \frac{E}{l} \alpha_{1}\left(1+\zeta \frac{1-f}{f}\right)+\lambda_{5, t+1}^{i} \beta(1-\rho) \zeta \frac{1-f}{f} \frac{E}{l} \alpha_{1} \\
& -\frac{1}{\nu_{1}} \frac{1-b}{b} \frac{C}{l} \frac{\zeta}{f}\left[\lambda_{9, t}^{i} \frac{f+\zeta(1-f)}{f+\zeta \rho(1-f)}-\beta \lambda_{9, t+1}^{i} \frac{\zeta(1-\rho)(1-f)}{f+\zeta \rho(1-f)}\right] \\
& +\lambda_{10, t}^{i} \frac{\alpha_{1}}{1-\alpha_{1}}-\beta \lambda_{10, t+1}^{i}(1-f)(1-\rho) \frac{\alpha_{1}}{1-\alpha_{1}}=0
\end{aligned}
$$

- $\tilde{e}_{t}^{j j}$ :

$$
\begin{aligned}
& \Gamma_{e}^{j j} \tilde{e}_{t}^{j j}+\Gamma_{e^{i i} e_{j} i \tilde{e}_{t}^{i j}}+\Gamma_{\theta e} \frac{f\left(1-\alpha_{1}\right)}{\zeta(1-f)} \tilde{\theta}_{t}^{j j}-\beta \Gamma_{\theta e} f(1-\rho)\left(1-\alpha_{1}\right)\left[\left(1-\alpha_{1}\right) \tilde{\theta}_{t+1}^{j j}+\alpha_{1} \tilde{\theta}_{t+1}^{j i}\right] \\
& +\frac{\lambda_{1, t}^{j j}}{\rho \gamma}-\beta \frac{1-\rho}{\rho \gamma}\left[\lambda_{1, t+1}^{j j}\left(1-f\left(1-\alpha_{1}\right)\right)-\lambda_{1, t+1}^{j i} f\left(1-\alpha_{1}\right)\right] \\
& +\lambda^{j} \frac{E^{\alpha}}{1-\alpha} \lambda_{3, t}^{j}\left(\frac{\alpha(1-\alpha)\left(1-\alpha_{1}\right)}{E^{\alpha}}-\frac{1-b}{b} \frac{C}{l} \frac{1-\zeta}{\nu_{1}} \frac{f+\zeta(1-f)}{f+\zeta \rho(1-f)}\right) \\
& +\lambda^{j} \frac{E^{\alpha}}{1-\alpha} \beta \lambda_{3, t+1}^{j} \frac{1-b}{b} \frac{C}{l} \frac{1-\zeta}{\nu_{1}} \frac{\zeta(1-\rho)(1-f)}{f+\zeta \rho(1-f)} \\
& +\lambda_{4, t}^{j}\left(\frac{1-\alpha}{E^{\alpha}}-\frac{\kappa}{q}\right) \frac{E}{C}\left(1-\alpha_{1}\right)+\lambda_{4, t+1}^{j} \beta(1-\rho) \frac{\kappa}{q} \frac{E}{C}\left(1-\alpha_{1}\right) \\
& -\lambda_{5, t}^{j} \frac{E}{l}\left(1-\alpha_{1}\right)\left(1+\zeta \frac{1-f}{f}\right)+\lambda_{5, t+1}^{j} \beta(1-\rho) \zeta \frac{1-f}{f} \frac{E}{l}\left(1-\alpha_{1}\right) \\
& +\frac{1}{\nu_{1}} \frac{1-b}{b} \frac{C}{l} \frac{\zeta}{f}\left[\lambda_{9, t}^{j} \frac{f+\zeta(1-f)}{f+\zeta \rho(1-f)}-\beta \lambda_{9, t+1}^{j} \frac{\zeta(1-\rho)(1-f)}{f+\zeta \rho(1-f)}\right] \\
& +\lambda_{10, t}^{j}-\beta \lambda_{10, t+1}^{j}(1-f)(1-\rho)=0
\end{aligned}
$$


- $e_{t}^{j i}$ :

$$
\begin{aligned}
& \Gamma_{e}^{j i} \tilde{e}_{t}^{j i}+\Gamma_{e^{i i} e^{j i}} \tilde{e}_{t}^{i i}+\Gamma_{\theta e} \frac{f \alpha_{1}}{\zeta(1-f)} \tilde{\theta}_{t}^{j i}-\beta \Gamma_{\theta e} f(1-\rho) \alpha_{1}\left[\left(1-\alpha_{1}\right) \tilde{\theta}_{t+1}^{j j}+\alpha_{1} \tilde{\theta}_{t+1}^{j i}\right] \\
& +\frac{\lambda_{1, t}^{j i}}{\rho \gamma}-\beta \frac{1-\rho}{\rho \gamma}\left[\lambda_{1, t+1}^{j i}\left(1-f \alpha_{1}\right)-\lambda_{1, t+1}^{j j} f \alpha_{1}\right]+\lambda_{3, t}^{i} \lambda^{i} \alpha \alpha_{1} \\
& +\lambda_{4, t}^{i}\left(\frac{1-\alpha}{E^{\alpha}}-\frac{\kappa}{q}\right) \frac{E}{C} \alpha_{1}+\lambda_{4, t+1}^{i} \beta(1-\rho) \frac{\kappa}{q} \frac{E}{C} \alpha_{1} \\
& -\lambda_{5, t}^{j} \frac{E}{l} \alpha_{1}\left(1+\zeta \frac{1-f}{f}\right)+\lambda_{5, t+1}^{j} \beta(1-\rho) \zeta \frac{1-f}{f} \frac{E}{l} \alpha_{1} \\
& -\frac{1}{\nu_{1}} \frac{1-b}{b} \frac{C}{l} \frac{\zeta}{f}\left[\lambda_{9, t}^{j} \frac{f+\zeta(1-f)}{f+\zeta \rho(1-f)}-\beta \lambda_{9, t+1}^{j} \frac{\zeta(1-\rho)(1-f)}{f+\zeta \rho(1-f)}\right] \\
& +\lambda_{10, t}^{j} \frac{\alpha_{1}}{1-\alpha_{1}}-\beta \lambda_{10, t+1}^{j}(1-f)(1-\rho) \frac{\alpha_{1}}{1-\alpha_{1}}=0
\end{aligned}
$$

- $\tilde{l}_{t}^{i}$ :

$$
-\frac{1-b}{b}\left(1+\frac{1}{\nu_{1}} \frac{l}{1-l}\right) \tilde{l}_{t}^{i}-\lambda_{3, t}^{i} \lambda^{i} \frac{E^{\alpha}}{1-\alpha}(1-\zeta) \frac{1-b}{b} \frac{C}{l}\left(1+\frac{1}{\nu_{1}} \frac{l}{1-l}\right)-\lambda_{5, t}^{i}=0
$$

- $\tilde{l}_{t}^{j}$ :

$$
-\frac{1-b}{b}\left(1+\frac{1}{\nu_{1}} \frac{l}{1-l}\right) \tilde{l}_{t}^{j}-\lambda_{3, t}^{j} \lambda^{j} \frac{E^{\alpha}}{1-\alpha}(1-\zeta) \frac{1-b}{b} \frac{C}{l}\left(1+\frac{1}{\nu_{1}} \frac{l}{1-l}\right)-\lambda_{5, t}^{j}=0
$$

- $\tilde{\omega}_{t}^{i}$ :

$$
-\left[\frac{1-\alpha_{1}}{\nu_{1}} \frac{E}{C} \frac{\kappa}{q} \frac{\rho \zeta(1-f)(1-\gamma)}{f+\zeta \rho(1-f)}\right] \rho\left(\tilde{\theta}_{t}^{i i}-\tilde{\theta}_{t}^{i j}\right)-\frac{\lambda_{1, t}^{i i}}{\gamma}+\frac{\lambda_{1, t}^{i j}}{\gamma} \frac{1-\alpha_{1}}{\alpha_{1}}=0
$$

- $\tilde{\omega}_{t}^{j}$ :

$$
-\left[\frac{1-\alpha_{1}}{\nu_{1}} \frac{E}{C} \frac{\kappa}{q} \frac{\rho \zeta(1-f)(1-\gamma)}{f+\zeta \rho(1-f)}\right] \rho\left(\tilde{\theta}_{t}^{j j}-\tilde{\theta}_{t}^{j i}\right)-\frac{\lambda_{1, t}^{j j}}{\gamma}+\frac{\lambda_{1, t}^{j i}}{\gamma} \frac{1-\alpha_{1}}{\alpha_{1}}=0
$$

- $\tilde{c}_{H, t}^{i}$ :

$$
\begin{aligned}
& -\frac{1-\alpha_{2}}{\nu_{2}} \widetilde{c}_{H, t}^{i}+\frac{E^{\alpha}}{1-\alpha} \frac{\kappa}{q \gamma} \frac{\lambda^{i}}{\nu_{2}}\left[\lambda_{3, t-1}^{i}(1-\rho)(1-f(1-\gamma))-\lambda_{3, t}^{i}\right] \\
& -\lambda_{4, t}^{i}\left(1-\alpha_{2}\right)-\frac{\lambda_{6, t}^{i}}{\nu_{2}}+\lambda_{7, t}^{i}\left(1-\alpha_{2}\right) \\
& -\frac{1}{\nu_{2}} \frac{\kappa}{q \gamma}(1-\gamma)\left(\lambda_{9, t}^{i}-\lambda_{9, t-1}^{i}(1-\rho)(1-f)\right) \\
& +\frac{1}{\nu_{2}} \frac{\kappa}{q \gamma}(1-\gamma)\left(\lambda_{9, t}^{j}-\lambda_{9, t-1}^{j}(1-\rho)(1-f)\right)=0
\end{aligned}
$$


- $\tilde{c}_{H, t}^{j}$ :

$$
\begin{aligned}
& -\frac{1-\alpha_{2}}{\nu_{2}} \tilde{c}_{H, t}^{j}+\frac{E^{\alpha}}{1-\alpha} \frac{\kappa}{q \gamma} \frac{\lambda^{j}}{\nu_{2}}\left[\lambda_{3, t-1}^{j}(1-\rho)(1-f(1-\gamma))-\lambda_{3, t}^{j}\right] \\
& -\lambda_{4, t}^{j}\left(1-\alpha_{2}\right)-\frac{\lambda_{6, t}^{j}}{\nu_{2}}+\lambda_{7, t}^{j}\left(1-\alpha_{2}\right) \\
& -\frac{1}{\nu_{2}} \frac{\kappa}{q \gamma}(1-\gamma)\left(\lambda_{9, t}^{j}-\lambda_{9, t-1}^{j}(1-\rho)(1-f)\right) \\
& +\frac{1}{\nu_{2}} \frac{\kappa}{q \gamma}(1-\gamma)\left(\lambda_{9, t}^{i}-\lambda_{9, t-1}^{i}(1-\rho)(1-f)\right)=0
\end{aligned}
$$

- $\tilde{c}_{F, t}^{i}$ :

$$
-\frac{\alpha_{2}}{\nu_{2}} \widetilde{c}_{F, t}^{i}-\lambda_{4, t}^{j} \alpha_{2}+\lambda_{7, t}^{i} \alpha_{2}=0
$$

- $\tilde{c}_{F, t}^{j}$ :

$$
-\frac{\alpha_{2}}{\nu_{2}} \tilde{c}_{F, t}^{j}-\lambda_{4, t}^{i} \alpha_{2}+\lambda_{7, t}^{j} \alpha_{2}=0
$$

We thus have a system of 21 constraints and 22 first-order conditions solving for 22 endogenous variables and 21 Lagrange multipliers. In addition, the first-order approximation of the efficient allocation is defined as follows:

1.

$$
\hat{l}_{t}^{i \star}=-\frac{E}{l}\left[\left(1-\alpha_{1}\right) \hat{e}_{t}^{i i \star}+\alpha_{1} \hat{e}_{t}^{i j \star}\right]-\frac{\zeta}{1-\zeta}\left[\left(1-\alpha_{1}\right) \hat{u}_{t}^{i i \star}+\alpha_{1} \hat{u}_{t}^{i j \star}\right]
$$

2.

$$
\hat{l}_{t}^{j \star}=-\frac{E}{l}\left[\left(1-\alpha_{1}\right) \hat{e}_{t}^{j j \star}+\alpha_{1} \hat{e}_{t}^{j i \star}\right]-\frac{\zeta}{1-\zeta}\left[\left(1-\alpha_{1}\right) \hat{u}_{t}^{j j \star}+\alpha_{1} \hat{u}_{t}^{j i \star}\right]
$$

3.

$$
\hat{c}_{t}^{i \star}=\left(1-\alpha_{2}\right) \hat{c}_{H, t}^{i \star}+\alpha_{2} \hat{c}_{F, t}^{i \star}
$$

4.

$$
\hat{c}_{t}^{j \star}=\left(1-\alpha_{2}\right) \hat{c}_{H, t}^{j \star}+\alpha_{2} \hat{c}_{F, t}^{j \star}
$$

5.

$$
\hat{y}_{t}^{i \star}=(1-\alpha)\left(\hat{a}_{t}^{i}+\hat{e}_{t}^{i \star}\right)=(1-\alpha)\left[\hat{a}_{t}^{i}+\left(1-\alpha_{1}\right) \hat{e}_{t}^{i i \star}+\alpha_{1} \hat{e}_{t}^{j i \star}\right]
$$


6.

$$
\hat{y}_{t}^{j \star}=(1-\alpha)\left(\hat{a}_{t}^{j}+\hat{e}_{t}^{j \star}\right)=(1-\alpha)\left[\hat{a}_{t}^{j}+\left(1-\alpha_{1}\right) \hat{e}_{t}^{j j \star}+\alpha_{1} \hat{e}_{t}^{i j \star}\right]
$$

7.

$$
\hat{e}_{t}^{i \star}=\left(1-\alpha_{1}\right) \hat{e}_{t}^{i i \star}+\alpha_{1} \hat{e}_{t}^{j i \star}
$$

8.

$$
\hat{e}_{t}^{j \star}=\left(1-\alpha_{1}\right) \hat{e}_{t}^{j j \star}+\alpha_{1} \hat{e}_{t}^{i j \star}
$$

9.

$$
\hat{q}_{t}^{i i \star}=(\gamma-1) \hat{\theta}_{t}^{i i \star}+\hat{\tau}_{t}^{i}
$$

10.

$$
\hat{q}_{t}^{i j \star}=(\gamma-1) \hat{\theta}_{t}^{i j \star}+\hat{\tau}_{t}^{j}
$$

11.

$$
\hat{q}_{t}^{j j \star}=(\gamma-1) \hat{\theta}_{t}^{j j \star}+\hat{\tau}_{t}^{j}
$$

12.

$$
\hat{q}_{t}^{j i \star}=(\gamma-1) \hat{\theta}_{t}^{j i \star}+\hat{\tau}_{t}^{i}
$$

13.

$$
\hat{f}_{t}^{i i \star}=\gamma \hat{\theta}_{t}^{i i \star}+\hat{\tau}_{t}^{i}
$$

14.

$$
\hat{f}_{t}^{i j \star}=\gamma \hat{\theta}_{t}^{i j \star}+\hat{\tau}_{t}^{j}
$$

15.

$$
\hat{f}_{t}^{j j \star}=\gamma \hat{\theta}_{t}^{j j \star}+\hat{\tau}_{t}^{j}
$$

16.

$$
\hat{f}_{t}^{j i \star}=\gamma \hat{\theta}_{t}^{j i \star}+\hat{\tau}_{t}^{i}
$$

17.

$$
\hat{e}_{t}^{i i \star}=(1-\rho) \hat{e}_{t-1}^{i i \star}+\rho\left(\hat{s}_{t}^{i i \star}+\hat{f}_{t}^{i i \star}\right)
$$


18.

$$
\hat{e}_{t}^{i j \star}=(1-\rho) \hat{e}_{t-1}^{i j \star}+\rho\left(\hat{s}_{t}^{i j \star}+\hat{f}_{t}^{i j \star}\right)
$$

19.

$$
\hat{e}_{t}^{j j \star}=(1-\rho) \hat{e}_{t-1}^{j j \star}+\rho\left(\hat{s}_{t}^{j j \star}+\hat{f}_{t}^{j j \star}\right)
$$

20.

$$
\hat{e}_{t}^{j i \star}=(1-\rho) \hat{e}_{t-1}^{j i \star}+\rho\left(\hat{s}_{t}^{j i \star}+\hat{f}_{t}^{j i \star}\right)
$$

21.

$$
\hat{u}_{t}^{i i \star}=\hat{s}_{t}^{i i \star}-\frac{f}{1-f} \hat{f}_{t}^{i i \star}
$$

22.

$$
\hat{u}_{t}^{i j \star}=\hat{s}_{t}^{i j \star}-\frac{f}{1-f} \hat{f}_{t}^{i j \star}
$$

23.

$$
\hat{u}_{t}^{j j \star}=\hat{s}_{t}^{j j \star}-\frac{f}{1-f} \hat{f}_{t}^{j j \star}
$$

24.

$$
\hat{u}_{t}^{j i \star}=\hat{s}_{t}^{j i \star}-\frac{f}{1-f} \hat{f}_{t}^{j i \star}
$$

25.

$$
\begin{array}{r}
\hat{y}_{t}^{i \star}=\quad \frac{C}{Y}\left\{\left(1-\alpha_{2}\right) \hat{c}_{H, t}^{i \star}+\alpha_{2} \hat{c}_{F, t}^{j \star}+\frac{\kappa}{q} \frac{E}{C}\left[\rho(1-\gamma) \hat{\theta}_{t}^{i \star}+\hat{e}_{t}^{i \star}-(1-\rho) \hat{e}_{t-1}^{i \star}\right]\right\} \\
=\quad \frac{C}{Y}\left\{\left(1-\alpha_{2}\right) \hat{c}_{H, t}^{i \star}+\alpha_{2} \hat{c}_{F, t}^{j \star}+\frac{\kappa}{q} \frac{E}{C} \rho(1-\gamma)\left(\left(1-\alpha_{1}\right) \hat{\theta}_{t}^{i i \star}+\alpha_{1} \hat{\theta}_{t}^{j i \star}\right)\right. \\
\left.+\frac{\kappa}{q} \frac{E}{C}\left(\left(1-\alpha_{1}\right) \hat{e}_{t}^{i i \star}+\alpha_{1} \hat{e}_{t}^{j i \star}\right)-\frac{\kappa}{q} \frac{E}{C}(1-\rho)\left(\left(1-\alpha_{1}\right) \hat{e}_{t-1}^{i i \star}+\alpha_{1} \hat{e}_{t-1}^{j i \star}\right)\right\}
\end{array}
$$

26.

$$
\begin{aligned}
\hat{y}_{t}^{j \star}= & \frac{C}{Y}\left\{\left(1-\alpha_{2}\right) \hat{c}_{H, t}^{j \star}+\alpha_{2} \hat{c}_{F, t}^{i \star}+\frac{\kappa}{q} \frac{E}{C}\left[\rho(1-\gamma) \hat{\theta}_{t}^{j \star}+\hat{e}_{t}^{j \star}-(1-\rho) \hat{e}_{t-1}^{j \star}\right]\right\} \\
= & \frac{C}{Y}\left\{\left(1-\alpha_{2}\right) \hat{c}_{H, t}^{j \star}+\alpha_{2} \hat{c}_{F, t}^{i \star}+\frac{\kappa}{q} \frac{E}{C} \rho(1-\gamma)\left(\left(1-\alpha_{1}\right) \hat{\theta}_{t}^{j j \star}+\alpha_{1} \hat{\theta}_{t}^{i j \star}\right)\right. \\
+ & \left.\frac{\kappa}{q} \frac{E}{C}\left(\left(1-\alpha_{1}\right) \hat{e}_{t}^{j j \star}+\alpha_{1} \hat{e}_{t}^{i j \star}\right)-\frac{\kappa}{q} \frac{E}{C}(1-\rho)\left(\left(1-\alpha_{1}\right) \hat{e}_{t-1}^{j j \star}+\alpha_{1} \hat{e}_{t-1}^{i j \star}\right)\right\}
\end{aligned}
$$


27.

$$
\hat{s}_{t}^{i j \star}=-\frac{(1-\rho) f}{\rho} \frac{1-\alpha_{1}}{\alpha_{1}} \hat{e}_{t-1}^{i i \star}-\frac{(1-\rho) f}{\rho} \hat{e}_{t-1}^{i j \star}-\frac{1-\alpha_{1}}{\alpha_{1}} \hat{s}_{t}^{i i \star}
$$

28.

$$
\hat{s}_{t}^{j i \star}=-\frac{(1-\rho) f}{\rho} \frac{1-\alpha_{1}}{\alpha_{1}} \hat{e}_{t-1}^{j j \star}-\frac{(1-\rho) f}{\rho} \hat{e}_{t-1}^{j i \star}-\frac{1-\alpha_{1}}{\alpha_{1}} \hat{s}_{t}^{j j \star}
$$

29.

$$
\hat{c}_{H, t}^{i \star}-\hat{c}_{F, t}^{j \star}=\nu_{2}\left(\hat{z}_{t}^{i}-\hat{z}_{t}^{j}\right)+\left(1-\nu_{2}\right)\left(\hat{c}_{t}^{i \star}-\hat{c}_{t}^{j \star}\right)
$$

30.

$$
\hat{c}_{H, t}^{j \star}-\hat{c}_{F, t}^{i \star}=\nu_{2}\left(\hat{z}_{t}^{j}-\hat{z}_{t}^{i}\right)+\left(1-\nu_{2}\right)\left(\hat{c}_{t}^{j \star}-\hat{c}_{t}^{i \star}\right)
$$

31.

$$
\begin{aligned}
& \frac{1-b}{b} \frac{C}{l}(1-\zeta)\left[\hat{c}_{t}^{i \star}-\hat{l}_{t}^{i \star}\left(1+\frac{1}{\nu_{1}} \frac{l}{1-l}\right)-\frac{1}{\nu_{1}} \hat{L}_{t}^{i i \star}-\hat{z}_{t}^{i}\right]= \\
& \left(\frac{1-\alpha}{E^{\alpha}}-\frac{\kappa}{q \gamma}\right) \frac{1}{\nu_{2}}\left(\hat{c}_{t}^{i \star}-\hat{c}_{H, t}^{i \star}\right)+\frac{1-\alpha}{E^{\alpha}} \hat{y}_{t}^{i \star}-\frac{1-\alpha}{E^{\alpha}}\left[\left(1-\alpha_{1}\right) \hat{e}_{t}^{i i \star}+\alpha_{1} \hat{e}_{t}^{j i \star}\right]-\frac{\kappa}{q \gamma} \hat{q}_{t}^{i i \star}+ \\
& \beta(1-\rho) \frac{\kappa}{q} \frac{1-(1-\gamma) f}{\gamma}\left[\hat{c}_{t}^{i \star}-\hat{c}_{t+1}^{i \star}-\hat{z}_{t}^{i}+\hat{z}_{t+1}^{i}+\frac{1}{\nu_{2}}\left(\hat{c}_{t+1}^{i \star}-\hat{c}_{H, t+1}^{i \star}\right)\right]+ \\
& \beta(1-\rho) \frac{\kappa}{q \gamma}(1-\gamma)(1-f) \hat{\theta}_{t+1}^{i i \star}
\end{aligned}
$$

32.

$$
\begin{aligned}
& \frac{1-b}{b} \frac{C}{l}(1-\zeta)\left[\hat{c}_{t}^{j \star}-\hat{l}_{t}^{j \star}\left(1+\frac{1}{\nu_{1}} \frac{l}{1-l}\right)-\frac{1}{\nu_{1}} \hat{L}_{t}^{j j \star}-\hat{z}_{t}^{j}\right]= \\
& \left(\frac{1-\alpha}{E^{\alpha}}-\frac{\kappa}{q \gamma}\right) \frac{1}{\nu_{2}}\left(\hat{c}_{t}^{j \star}-\hat{c}_{H, t}^{j \star}\right)+\frac{1-\alpha}{E^{\alpha}} \hat{y}_{t}^{j \star}-\frac{1-\alpha}{E^{\alpha}}\left[\left(1-\alpha_{1}\right) \hat{e}_{t}^{j j \star}+\alpha_{1} \hat{e}_{t}^{i j \star}\right]-\frac{\kappa}{q \gamma} \hat{q}_{t}^{j j \star}+ \\
& \beta(1-\rho) \frac{\kappa}{q} \frac{1-(1-\gamma) f}{\gamma}\left[\hat{c}_{t}^{j \star}-\hat{c}_{t+1}^{j \star}-\hat{z}_{t}^{j}+\hat{z}_{t+1}^{j}+\frac{1}{\nu_{2}}\left(\hat{c}_{t+1}^{j \star}-\hat{c}_{H, t+1}^{j \star}\right)\right]+ \\
& \beta(1-\rho) \frac{\kappa}{q \gamma}(1-\gamma)(1-f) \hat{\theta}_{t+1}^{j j \star}
\end{aligned}
$$

33.

$$
\begin{aligned}
& \frac{1-b}{b} \frac{C}{l}(1-\zeta)\left[\hat{c}_{t}^{i \star}-\hat{l}_{t}^{i \star}\left(1+\frac{1}{\nu_{1}} \frac{l}{1-l}\right)-\frac{1}{\nu_{1}} \hat{L}_{t}^{i j \star}-\hat{z}_{t}^{i}\right]= \\
& \left(\frac{1-\alpha}{E^{\alpha}}-\frac{\kappa}{q \gamma}\right) \frac{1}{\nu_{2}}\left(\hat{c}_{t}^{i \star}-\hat{c}_{F, t}^{i \star}\right)+\frac{1-\alpha}{E^{\alpha}} \hat{y}_{t}^{j \star}-\frac{1-\alpha}{E^{\alpha}}\left[\left(1-\alpha_{1}\right) \hat{e}_{t}^{j j \star}+\alpha_{1} \hat{e}_{t}^{i j \star}\right]-\frac{\kappa}{q \gamma} \hat{q}_{t}^{i j \star}+ \\
& \beta(1-\rho) \frac{\kappa}{q} \frac{1-(1-\gamma) f}{\gamma}\left[\hat{c}_{t}^{i \star}-\hat{c}_{t+1}^{i \star}-\hat{z}_{t}^{i}+\hat{z}_{t+1}^{i}+\frac{1}{\nu_{2}}\left(\hat{c}_{t+1}^{i \star}-\hat{c}_{F, t+1}^{i \star}\right)\right]+ \\
& \beta(1-\rho) \frac{\kappa}{q \gamma}(1-\gamma)(1-f) \hat{\theta}_{t+1}^{i j \star}
\end{aligned}
$$


34.

$$
\begin{aligned}
& \frac{1-b}{b} \frac{C}{l}(1-\zeta)\left[\hat{c}_{t}^{j \star}-\hat{l}_{t}^{j \star}\left(1+\frac{1}{\nu_{1}} \frac{l}{1-l}\right)-\frac{1}{\nu_{1}} \hat{L}_{t}^{j i \star}-\hat{z}_{t}^{j}\right]= \\
& \left(\frac{1-\alpha}{E^{\alpha}}-\frac{\kappa}{q \gamma}\right) \frac{1}{\nu_{2}}\left(\hat{c}_{t}^{j \star}-\hat{c}_{F, t}^{j \star}\right)+\frac{1-\alpha}{E^{\alpha}} \hat{y}_{t}^{i \star}-\frac{1-\alpha}{E^{\alpha}}\left[\left(1-\alpha_{1}\right) \hat{e}_{t}^{i i \star}+\alpha_{1} \hat{e}_{t}^{j i \star}\right]-\frac{\kappa}{q \gamma} \hat{q}_{t}^{j i \star}+ \\
& \beta(1-\rho) \frac{\kappa}{q} \frac{1-(1-\gamma) f}{\gamma}\left[\hat{c}_{t}^{j \star}-\hat{c}_{t+1}^{j \star}-\hat{z}_{t}^{j}+\hat{z}_{t+1}^{j}+\frac{1}{\nu_{2}}\left(\hat{c}_{t+1}^{j \star}-\hat{c}_{F, t+1}^{j \star}\right)\right]+ \\
& \beta(1-\rho) \frac{\kappa}{q \gamma}(1-\gamma)(1-f) \hat{\theta}_{t+1}^{j i \star}
\end{aligned}
$$

35.

$$
\hat{L}_{t}^{i i \star}=\frac{f+\zeta(1-f)}{f+\rho \zeta(1-f)} \hat{e}_{t}^{i i \star}-\frac{\zeta(1-\rho)(1-f)}{f+\rho \zeta(1-f)} \hat{e}_{t-1}^{i i \star}-\frac{\zeta \rho \gamma}{f+\rho \zeta(1-f)} \hat{\theta}_{t}^{i i \star}-\frac{\zeta \rho}{f+\zeta \rho(1-f)} \hat{\omega}_{t}^{i}
$$

36.

$$
\hat{L}_{t}^{i j \star}=\frac{f+\zeta(1-f)}{f+\rho \zeta(1-f)} \hat{e}_{t}^{i j \star}-\frac{\zeta(1-\rho)(1-f)}{f+\rho \zeta(1-f)} \hat{e}_{t-1}^{i j \star}-\frac{\zeta \rho \gamma}{f+\rho \zeta(1-f)} \hat{\theta}_{t}^{i j \star}-\frac{\zeta \rho}{f+\zeta \rho(1-f)} \hat{\omega}_{t}^{j}
$$

37.

$$
\hat{L}_{t}^{j j \star}=\frac{f+\zeta(1-f)}{f+\rho \zeta(1-f)} \hat{e}_{t}^{j j \star}-\frac{\zeta(1-\rho)(1-f)}{f+\rho \zeta(1-f)} \hat{e}_{t-1}^{j j \star}-\frac{\zeta \rho \gamma}{f+\rho \zeta(1-f)} \hat{\theta}_{t}^{j j \star}-\frac{\zeta \rho}{f+\zeta \rho(1-f)} \hat{\omega}_{t}^{j}
$$

38.

$$
\hat{L}_{t}^{j i \star}=\frac{f+\zeta(1-f)}{f+\rho \zeta(1-f)} \hat{e}_{t}^{j i \star}-\frac{\zeta(1-\rho)(1-f)}{f+\rho \zeta(1-f)} \hat{e}_{t-1}^{j i \star}-\frac{\zeta \rho \gamma}{f+\rho \zeta(1-f)} \hat{\theta}_{t}^{j i \star}-\frac{\zeta \rho}{f+\zeta \rho(1-f)} \hat{\omega}_{t}^{i}
$$

39.

$$
\frac{1}{\nu_{1}}\left(\hat{L}_{t}^{i i \star}-\hat{L}_{t}^{i j \star}\right)+\left(\hat{\theta}_{t}^{i i \star}-\hat{\theta}_{t}^{i j \star}\right)+\frac{1}{\nu_{2}}\left(\hat{c}_{t}^{i \star}-\hat{c}_{H, t}^{i \star}-\hat{c}_{t}^{j \star}+\hat{c}_{H, t}^{j \star}\right)=0
$$

40.

$$
\frac{1}{\nu_{1}}\left(\hat{L}_{t}^{j j \star}-\hat{L}_{t}^{j i \star}\right)+\left(\hat{\theta}_{t}^{j j \star}-\hat{\theta}_{t}^{j i \star}\right)+\frac{1}{\nu_{2}}\left(\hat{c}_{t}^{j \star}-\hat{c}_{H, t}^{j \star}-\hat{c}_{t}^{i \star}+\hat{c}_{H, t}^{i \star}\right)=0
$$

41.

$$
\hat{\mathrm{q}}_{t}^{\star}=\frac{2 \alpha_{2}-1}{\alpha_{2}} \frac{1}{\nu_{2}}\left(\hat{c}_{t}^{i \star}-\hat{c}_{H, t}^{i \star}\right)
$$

